\title{
The role of amyloid beta 4-42 in the etiology of Alzheimer's disease
}

\section{Doctoral Thesis}

In partial fulfillment of the requirements for the degree

"Doctor rerum naturalium (Dr. rer. nat.)"

in the Molecular Medicine Study Program

at the Georg-August University Göttingen

submitted by

\section{Yvonne Bouter}

born in

Peine, Germany

Göttingen 2014 


\section{Members of the thesis commitee:}

Prof. Dr. Thomas A. Bayer (Supervisor)

Department for Psychiatry

Division of Molecular Psychiatry

University Medical Center Göttingen

Göttingen

Prof. Dr. Uwe-Karsten Hanisch

Dept. of Neuropathology

University Medical Center Göttingen

Göttingen

Prof. Dr. Dr. Hannelore Ehrenreich

Division of Clinical Neurosciences

Max Planck Institute of Experimental Medicine

Göttingen

Date of the oral examination: 12.11.2014 


\section{Declaration:}

Here I declare that my doctoral thesis entitled "The role of amyloid beta 4-42 in the etiology of Alzheimer's disease" has been written independently with no other sources and aids than quoted.

Yvonne Bouter

Göttingen, September 2014 


\section{List of Publications:}

Parts of this thesis have been published:

\section{Original articles:}

Bouter, Y., Dietrich, K., Wittnam, J.L., Rezaei-Ghaleh, N., Pillot, T., Papot-Couturier, S., Lefebvre, T., Sprenger, F., Wirths, O., Zweckstetter, M., and Bayer T.A. (2013). Ntruncated amyloid $\beta$ (A $\beta$ ) 4-42 forms stable aggregates and induces acute and longlasting behavioral deficits. Acta Neuropathol. 126, 189-205.

Bouter, Y., Kacprowski, T., Weissmann, R., Dietrich, K., Borgers, H., Brauß, A., Sperling, C., Wirths, O., Albrecht, M., Jensen, L.R., Kuss, A.W., and Bayer T.A. (2014). Deciphering the Molecular Profile of Plaques, Memory Decline and Neuron Loss in Two Mouse Models for Alzheimer's Disease by Deep Sequencing. Front. Aging Neurosci. 6 .

Antonios, G., Saiepour, N., Bouter, Y., Richard, B.C., Paetau, A., Verkkoniemi-Ahola, A., Lannfelt, L., Ingelsson, M., Kovacs, G.G., Pillot, T., Wirths, O., and Bayer T.A. (2013). Ntruncated Abeta starting with position four: early intraneuronal accumulation and rescue of toxicity using NT4X-167, a novel monoclonal antibody. Acta Neuropathol Commun 1, 56.

Guzmán, E.A., Bouter, Y., Richard, B.C., Lannfelt, L., Ingelsson, M., Paetau, A., Verkkoniemi-Ahola, A., Wirths, O., and Bayer T.A. (2014). Abundance of A $\beta 5-x$ like immunoreactivity in transgenic 5XFAD, APP/PS1KI and 3xTG mice, sporadic and familial Alzheimer's disease. Mol Neurodegener 9, 13.

\section{Abstracts:}

Bouter, Y., Dietrich, K., Wittnam, J.L., Pillot, T., Papot-Couturier, S., Lefebvre, T., Sprenger, F., Wirths, O., Zweckstetter, M., and Bayer T.A. (2013). Tg4-42: A new mouse model of Alzheimer's disease - $N$-truncated amyloid $\beta(A \beta)$ 4-42 induces severe neuron loss and behavioral deficits. Alzheimer's and Dementia 9, P498.

Dietrich, K., Bouter, Y., Wittnam, J.L., Pillot, T., Papot-Couturier, S., Lefebvre, T., Sprenger, F., Wirths, O., Janc O.A., Müller M., Zweckstetter, M., and Bayer T.A. (2013). Tg4-42: A new mouse model of Alzheimer's disease-N-truncated beta-amyloid 4-42 affects memory decline and synaptic plasticity. Alzheimer's and Dementia 9, P498.

Bayer T.A., Bouter Y., Kacprowski T, Sperling C, Albrecht M, Weißmann R, et al. (2014). Deep sequencing unravels the molecular signatures in two different mouse models for Alzheimer disease. Medizinische Genetik. 26, 107. 


\section{Contents}

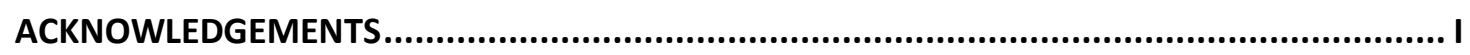

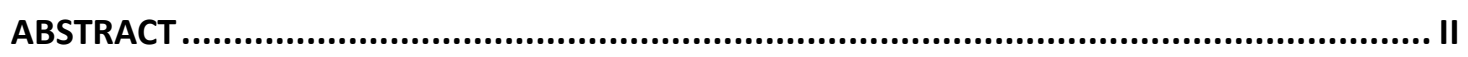

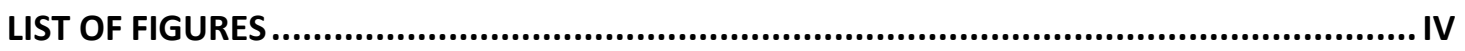

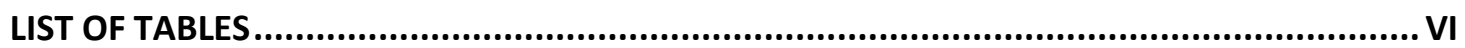

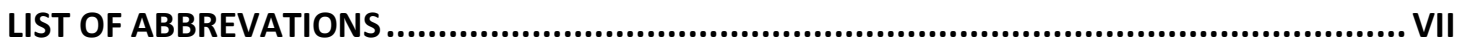

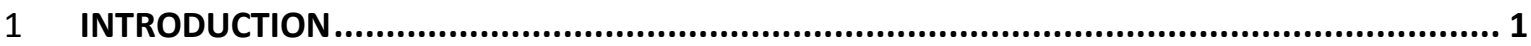

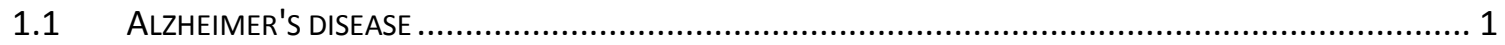

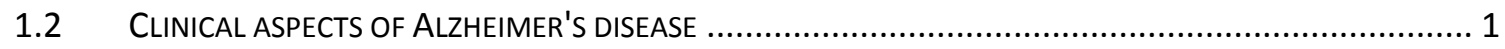

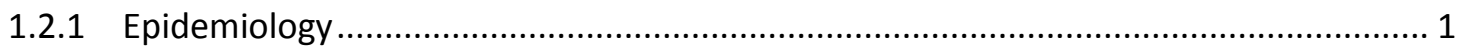

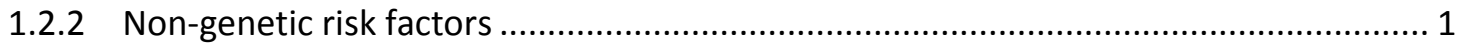

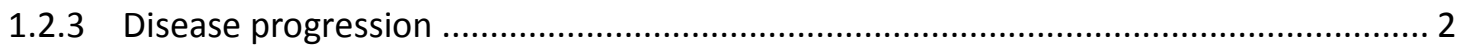

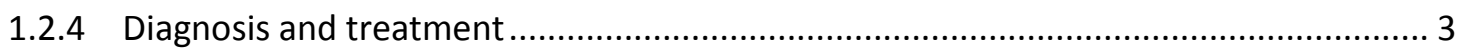

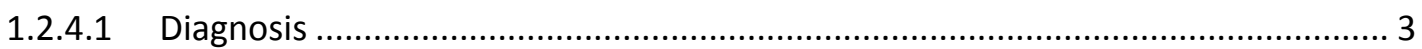

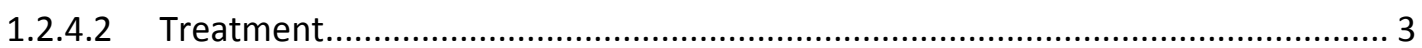

1.3 NeURopathologiCAL HALLMARKS OF ALZHEIMER'S DISEASE ...................................................... 4

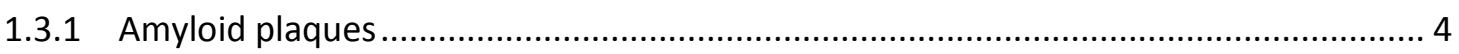

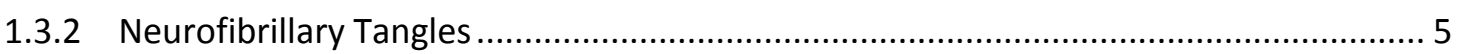

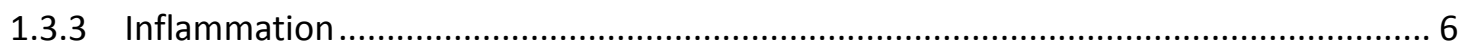

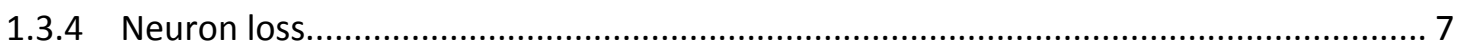

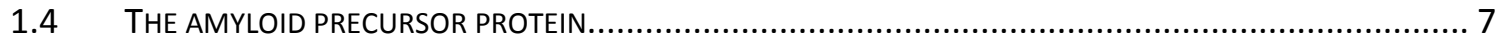

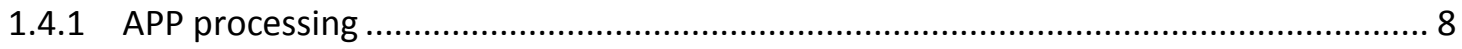

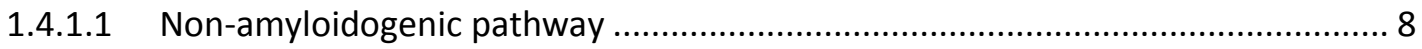

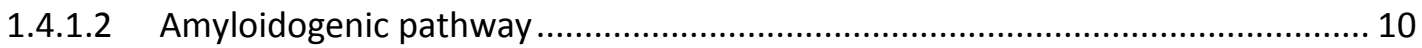

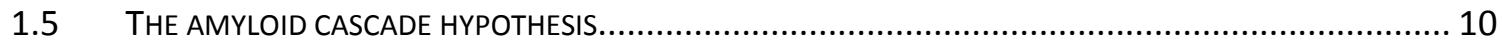

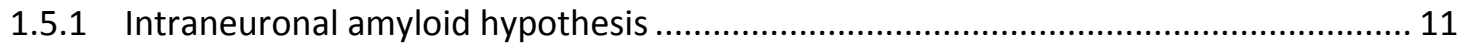

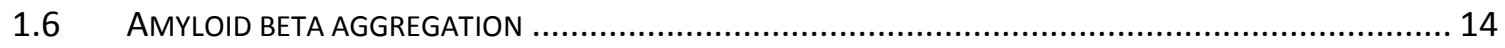

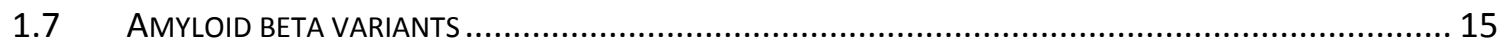

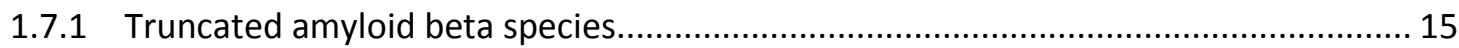


1.7.1.1 Amyloid beta $4-42$.

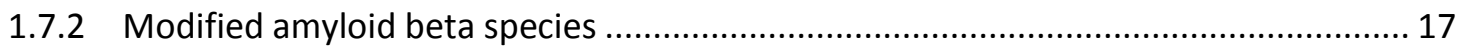

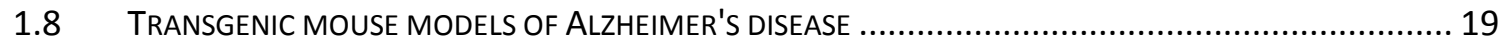

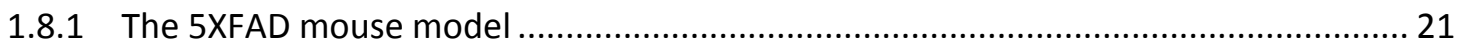

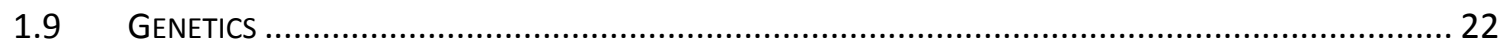

1.9.1 From genome-wide association studies to next-generation sequencing ................... 24

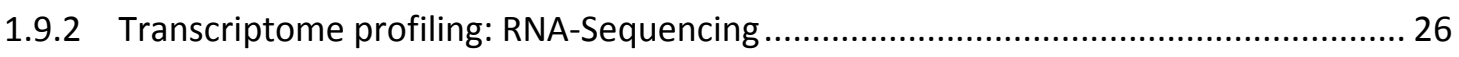

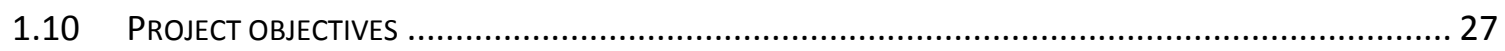

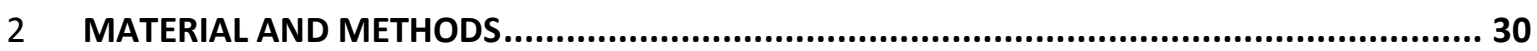

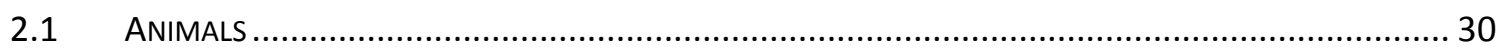

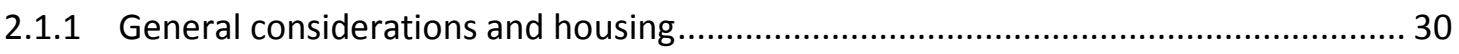

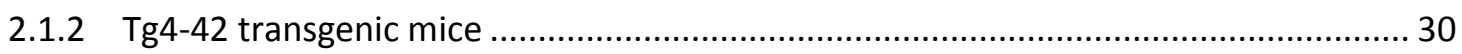

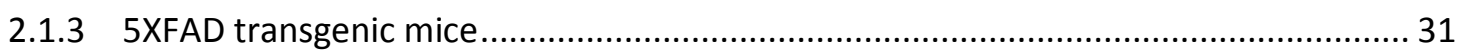

2.1.4 Preparation of mouse brain tissue for biochemistry ................................................... 32

2.1.5 Preparation of mouse brain tissue for immunohistochemistry ................................. 32

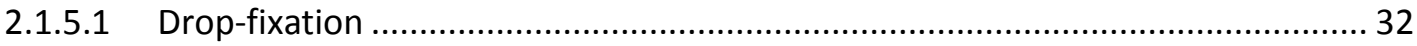

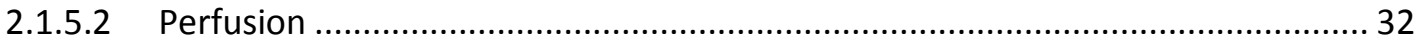

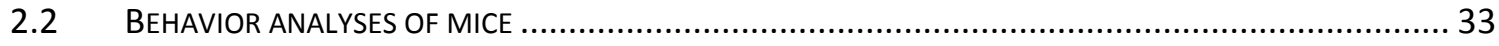

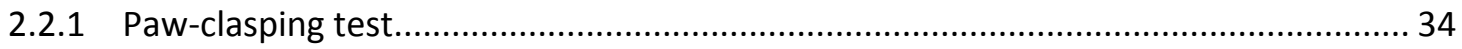

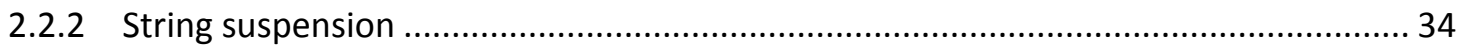

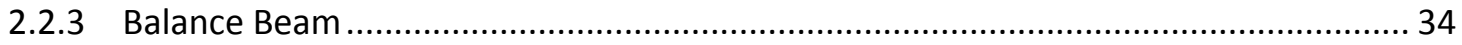

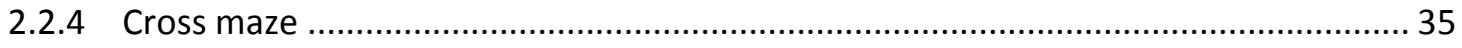

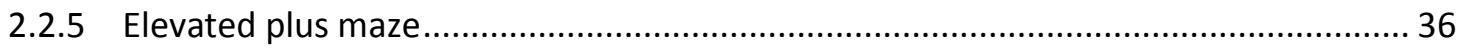

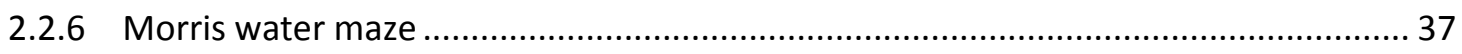

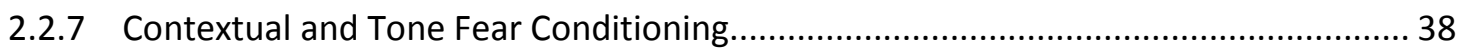

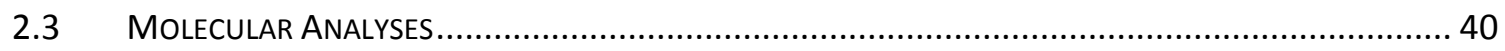

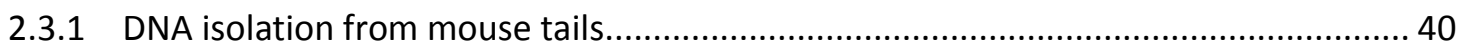

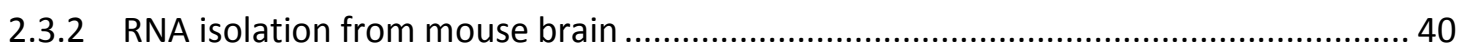

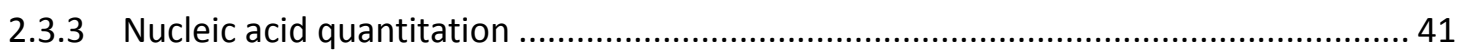

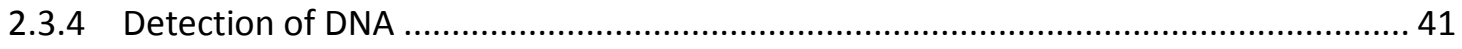

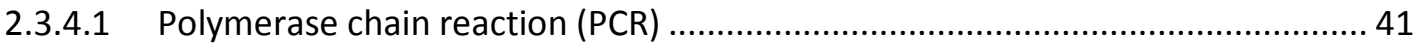

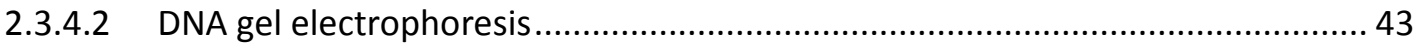

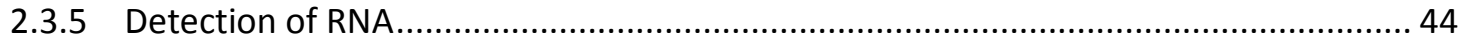

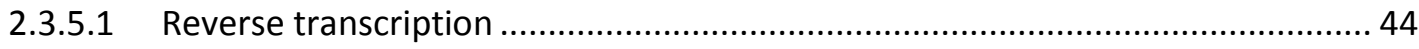




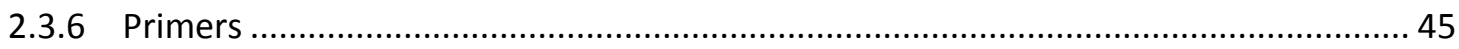

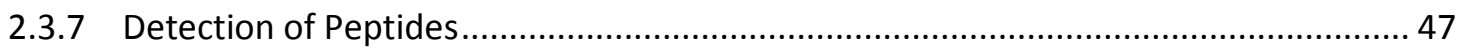

2.3.7.1 Monomerization of Synthetic Peptides............................................................ 47

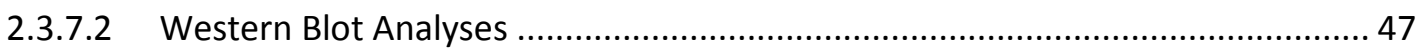

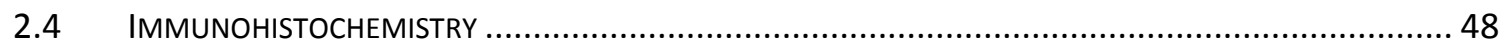

2.4.1 Paraffin embedding and sectioning of mouse brain ................................................. 48

$2.4 .2 \quad 3.3$ '-Diaminobenzidine (DAB) immunohistochemistry.............................................. 49

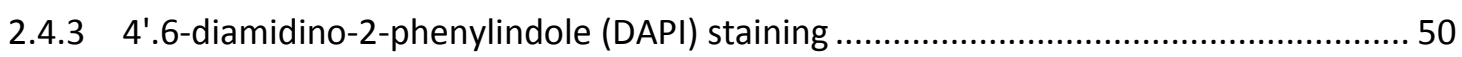

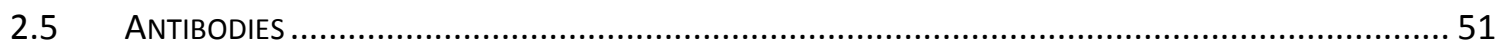

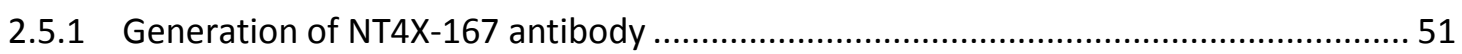

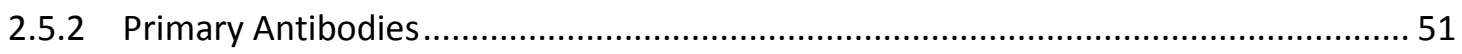

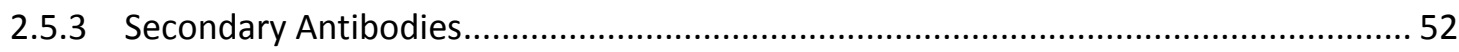

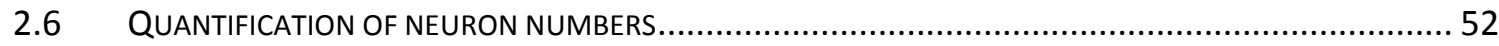

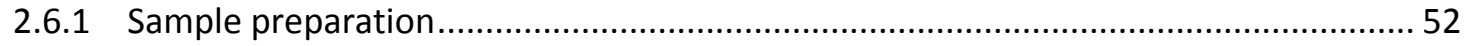

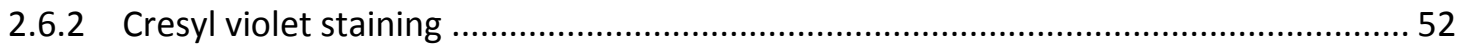

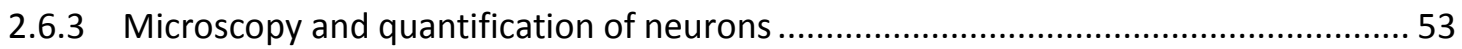

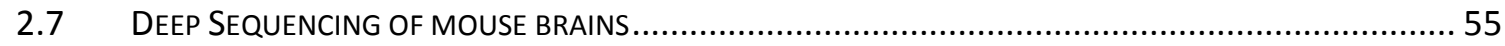

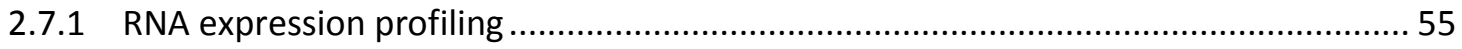

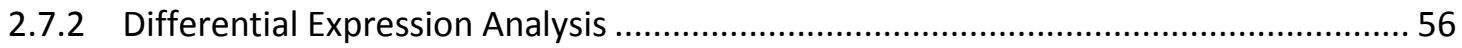

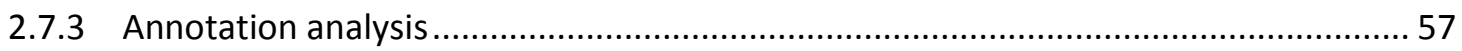

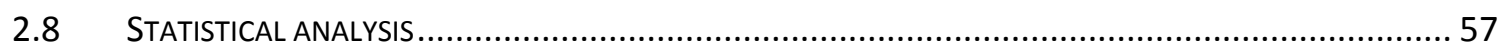

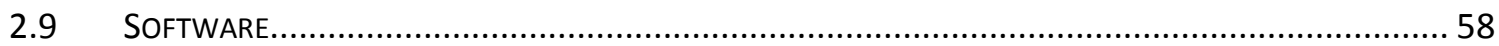

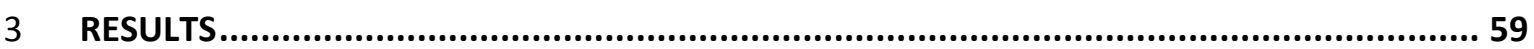

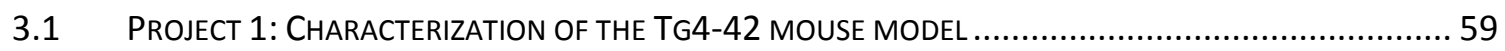

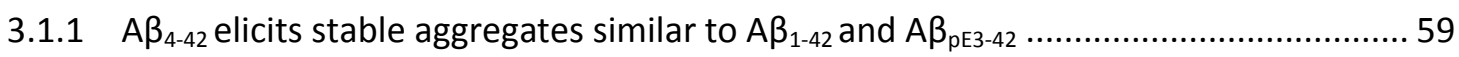

3.1.2 Early intraneuronal $A \beta_{4-x}$ accumulation in 5XFAD transgenic mice............................... 60

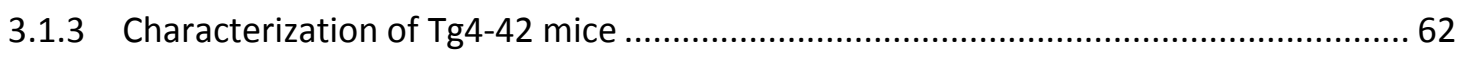

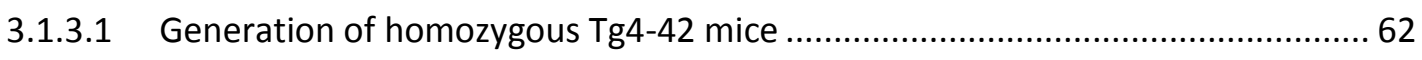

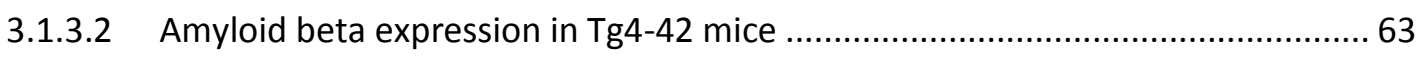

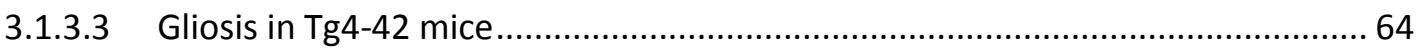

3.1.3.4 Physical and behavioral characterization of Tg4-42 mice .................................. 65

3.1.3.5 Long-term exposure to $\mathrm{N}$-truncated $A \beta_{4-42}$ induces neuron loss........................... 75 
3.2 PROJECT 2: DECIPHERING THE MOLECULAR PROFILE OF PLAQUES, MEMORY DECLINE AND NEURON LOSS IN TG4-42 AND 5XFAD MICE BY DEEP SEQUENCING ................................................ 78

3.2.1 Amyloid beta accumulation in Tg4-42 and 5XFAD mice ................................... 78

3.2.2 Tg4-42 and 5XFAD mice display spatial memory deficits..................................... 79

3.2.3 Tg4-42 and 5XFAD mice exhibit decreased contextual learning............................. 83

3.2.4 5XFAD mice show impaired tone learning ................................................... 83

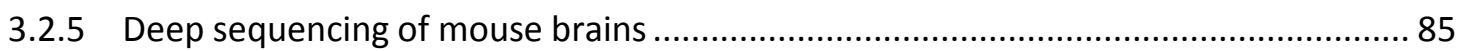

3.2.5.1 Deep sequencing (RNASeq) identified over-expressed transgenes ................... 85

3.2.5.2 Differentially regulated genes in Tg4-42 and 5XFAD mice............................. 86

3.2.5.3 Gene expression in young Tg4-42 and 5XFAD mice .................................. 87

3.2.5.4 Gene expression in aged Tg4-42 mice.................................................... 88

3.2.5.5 Gene expression in aged 5XFAD mice ................................................ 88

3.2.5.6 Genes similarly expressed in both aged Tg4-42 and 5XFAD mice....................88

3.2.5.7 Genes similarly expressed in both young and aged 5XFAD mice.....................89

3.2.5.8 Validation of differentially expressed genes identified by RNA-Seq using

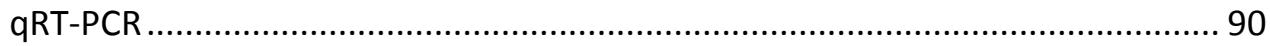

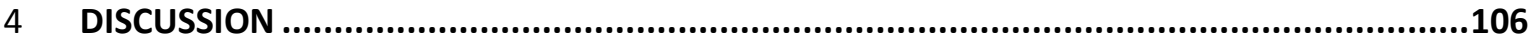

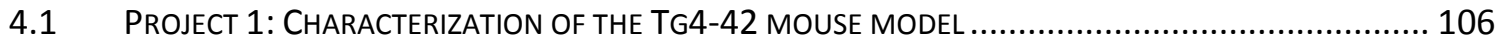

4.1.1 $A \beta_{4-x}$ precedes $A \beta_{p E 3-x}$ accumulation in 5XFAD mice ..................................... 107

4.1.2 Intraneuronal $A \beta$ expression in Tg4-42 mice ................................................ 108

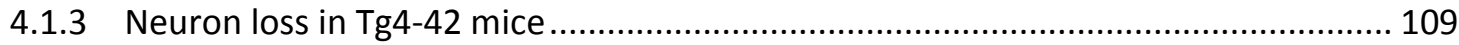

4.1.4 Enhanced inflammation in Tg4-42 mice....................................................... 111

4.1.5 No weight loss in Tg4-42 mice........................................................... 112

4.1.6 No motor deficits or anxiety-behavior in Tg4-42 mice ...................................... 113

4.1.7 Age-dependant memory deficits in Tg4-42 mice .............................................. 114

4.1.8 Summary - Is Tg4-42 a valid model for AD? ................................................... 118

4.2 PROJECT 2: DECIPHERING THE MOLECULAR PROFILE OF PLAQUES, MEMORY DECLINE AND NEURON LOSS

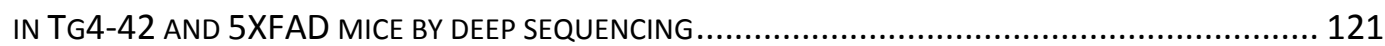

4.2.1 Advantages of RNA Deep Sequencing ........................................................ 121

4.2.2 Comparison of Tg4-42 and 5XFAD mice ...................................................... 122

4.2.3 Learning and memory deficits in 5XFAD and Tg4-42 mice.............................. 124

4.2.4 Deep sequencing in 5XFAD and Tg4-42 mice ........................................... 124

4.2.5 Differentially expressed genes in young 5XFAD and Tg4-42 mice ........................ 125

4.2.6 Common molecular signature of Tg4-42 and 5XFAD mice ................................ 125 
4.2.7 Differentially expressed genes in aged Tg4-42 mice.

4.2.8 Differentially expressed genes in aged 5XFAD mice ............................................... 129

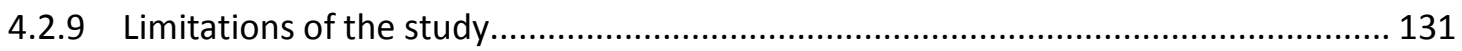

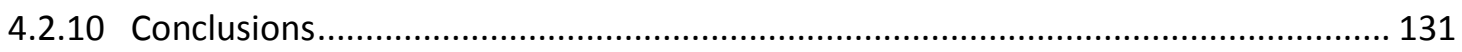

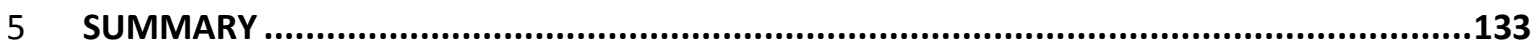

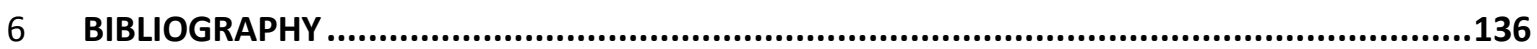

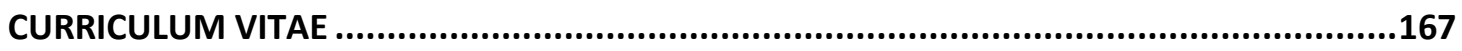




\section{Acknowledgments}

First and foremost, I want to thank my supervisor Prof. Dr. Thomas A. Bayer for giving me the opportunity to carry out this thesis in his laboratory. I am sincerely grateful to his mentorship and encouragement during my Ph.D. I would like to thank him for always taking the time to discuss my projects and letting me grow to become an independent researcher. I would also like to thank PD Dr. Oliver Wirths for discussing and supporting my work.

Furthermore, I would also like to thank my thesis committee Prof. Dr. Dr. Hannelore Ehrenreich and Prof. Dr. Uwe-Karsten Hanisch for agreeing to be members of my PhD thesis committee and providing me with encouraging and insightful feedback.

I'm very grateful to the Alzheimer Stiftung Göttingen for awarding me with 'Inge und Fritz Kleekamm Forschungspreis' and thereby supported my research work immensely.

For their companionship during the past three years, I would like to thank all current and former members of the Bayer lab. In particular I like to thank Sadeem Jawhar, Anika Saul, Antje Hillmann and Jessica Wittnam. I am especially grateful to Katharina Dietrich for her friendship and our endless scientific and non-scientific discussions.

I appreciate the opportunity that all the rotation, master and MD students of the Bayer lab have given me to teach and learn at the same time. In particular I like to thank Anna Greda, Freddy Sprenger, Henning Borgers and Andreas Brauß.

My sincerest thanks go to Petra Tucholla for always being available for technical and logistic support. I would also like to thank Nina Karbe and the other members of the UMG animal facility. In addition, I like to thank my collaborators outside the group. Especially I like to thank Tim Kacprowski for his bioinformatics expertise and Robert Weissmann who taught me the secrets of deep sequencing. Furthermore, I like to thank Michael Krusche for the excel makro and Shreya Sawant for proofreading my thesis.

This thesis would have not be possible without the love and support of my family. I like to thank my mother for all her love and encouragement. Especially, I like to thank my sister and best friend Caroline for her invaluable support during the last three years. This thesis is dedicated in loving memory to my father. 


\section{Abstract}

$N$-truncated $A \beta_{\text {4-42 }}$ has been identified as a particular abundant $A \beta$ species in the hippocampus and cortex of $A D$ patients. However, relatively little is known about the contribution of $A \beta_{4-42}$ to the development and progression of $A D$. In order to study the effects of chronic exposure of $A \beta_{4-42}$ the transgenic mouse model Tg4-42 was generated. The Tg4-42 mouse model, expressing exclusively human $A \beta_{4-42}$, allows to investigate the neurotoxic effects of $A \beta_{4-42}$ in vivo.

In the present work, it could be shown that $\mathrm{Tg} 4-42$ mice show region-specific intraneuronal accumulation of $A \beta$ accompanied by gliosis. Tg4-42 mice showed strong intraneuronal $A \beta$ immunoreactivity predominantly in the CA1 pyramidal cell layer of the hippocampus. Strikingly, transgenic mice expressing $A \beta_{4-42}$ develop a massive $C A 1$ neuron loss. The progressive neuron loss in the hippocampus of Tg4-42 mice correlates strongly with intraneuronal $A \beta_{4-42}$. The behavior analysis of Tg4-42 mice revealed hippocampus-dependant memory deficits similar to AD patients. Importantly, the observed memory deficits in Tg4-42 mice are not confounded by motor impairments or abnormal anxiety. Mice are profoundly impaired in their spatial reference memory assessed in the Morris water maze. Moreover, aged Tg4-42 showed a decline in contextual fear memory. The over-expression of $A \beta_{4-42}$ in this mouse model induces severe age-dependant memory deficits that can be attributed to the massive neuron loss in the hippocampus. The transgenic mouse model Tg4-42 is unique as it harbors no mutations in the $A \beta$ sequence. In summary, $\operatorname{Tg} 4-42$ is a valid AD mouse model showing key features of sporadic AD.

One of the central research questions on the etiology of $A D$ is the elucidation of the molecular signatures triggered by the amyloid cascade of pathological events. Next generation sequencing allows the identification of genes involved in disease processes in an unbiased manner. The gene expression profiles of Tg4-42 mice were compared to the widely used 5XFAD mouse model using next-generation sequencing. The comparison with $5 X F A D$, an established plaque-developing $A D$ mouse model, revealed a remarkable overlap in the molecular signature. The jointly differentially expressed genes might indicate common pathways that are involved in the comparable learning and memory decline apparent at twelve months of age in both transgenic models. The pool of genes that showed differential expression exclusively in Tg4-42 is likely associated to soluble $A \beta$ as no extracellular plaques are found in this model. In addition, the robust CA1 neuron loss could also contribute to the differential expression profile. As most of the genes with expression levels exclusively altered in 5XFAD mice belong to inflammation-associated 
pathways, it can be concluded that the majority of these genes are regulated in connection with plaque formation in this model and are not associated with neuron loss and memory decline. Furthermore, the deep sequencing approach identified a plethora of genes that have so far not been linked to $A D$ and therefore open up new avenues of research into the etiology of this devastating neurodegenerative disorder.

In summary, the results of this thesis demonstrate $A \beta_{4-42}$ as a toxic $A \beta$ variant that likely plays an important role in triggering $A D$ pathology. 


\section{List of Figures}

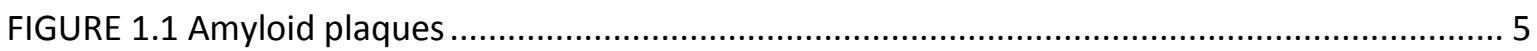

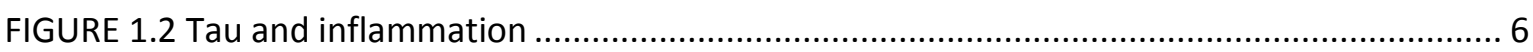

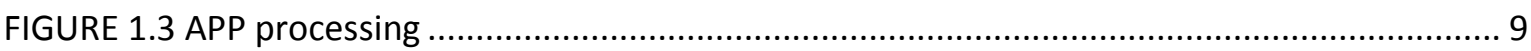

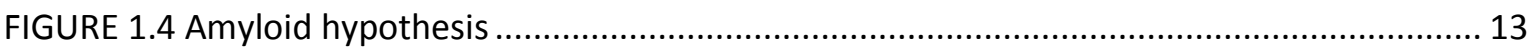

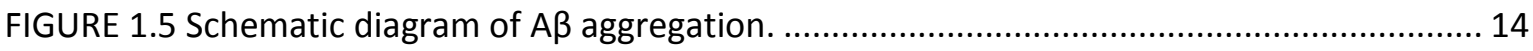

FIGURE 1.6 Schematic overview of proteases and cleavage sites involved in generation of

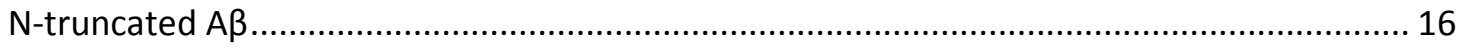

FIGURE 1.7 Schematic overview of post-translational modifications in A $\beta 1-42$............................ 18

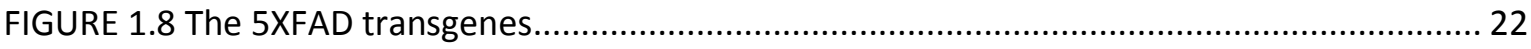

FIGURE 1.9 Schematic overview of the projects presented in this thesis. ..................................... 29

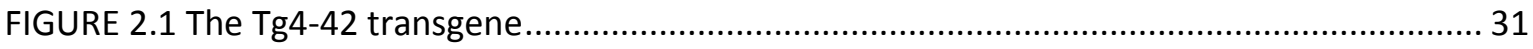

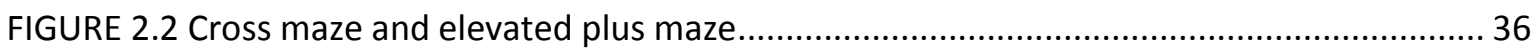

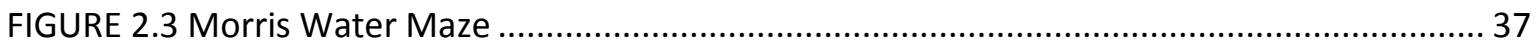

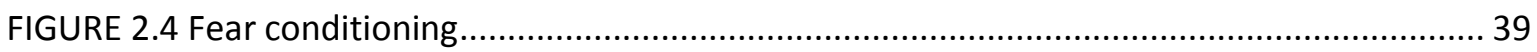

FIGURE 2.5 Procedure of design-based stereology of the CA1_....................................................... 53

FIGURE 3.1 Freshly dissolved $A \beta_{1-42}, A \beta_{p E 3-42}, A \beta_{4-38}, A \beta_{4-40}$ and $A \beta_{4-42}$ rapidly formed stable

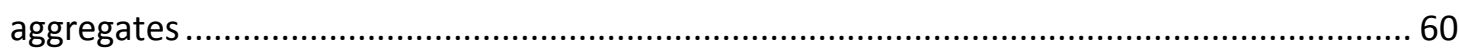

FIGURE 3.2 Immunohistochemical staining of cortical sections of 5XFAD transgenic mice ............. 61

FIGURE 3.3 Identification of potentially homozygous Tg4-42 mice through qRT-PCR .....................62

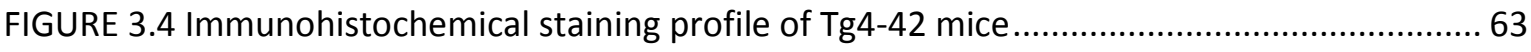

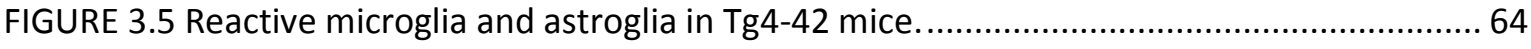

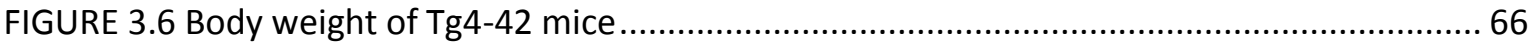

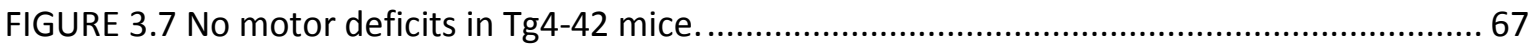

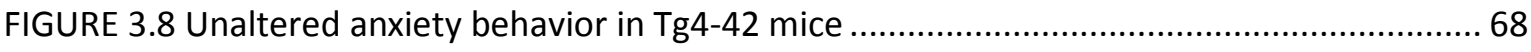

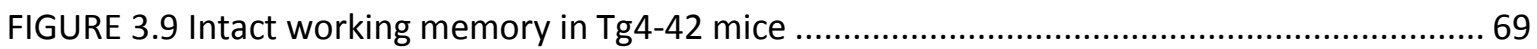

FIGURE 3.10 Cued training of the water maze reveales that Tg4-42 mice exhibit intact eyesight and the motor abilities to perform the test...................................................................... 71

FIGURE 3.11 Spatial learning was assessed in the acquisition training of the Morris water maze 72

FIGURE 3.12 Spatial reference memory deficits in aged Tg4-42 and Tg4-42hom mice shown in

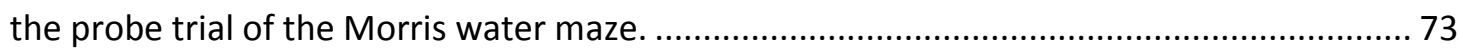


FIGURE 3.13 Impaired contextual conditioning in Tg4-42 mice

FIGURE 3.14 Age- and dose-dependent neuron loss in hippocampus of Tg4-42 mice. 76

FIGURE 3.15 Immunohistochemical staining of 5XFAD and Tg4-42 mice.

FIGURE 3.16 Cued training of the water maze reveals that Tg4-42 and 5XFAD exhibit intact eyesight and the motor abilities to perform the test.

FIGURE 3.17 Spatial learning deficits in aged Tg4-42 and 5XFAD shown in the acquisition training of the Morris water maze

FIGURE 3.18 Spatial reference memory deficits in aged Tg4-42 and 5XFAD mice shown in the probe trial of the Morris water maze

FIGURE 3.19 Impaired contextual conditioning in Tg4-42 and 5XFAD mice.

FIGURE 3.20 Impaired tone conditioning in 5XFAD mice.

FIGURE 3.21 Volcano plots of the significant gene expression changes in Tg4-42 and 5XFAD mice

FIGURE 3.22 Venn diagram analysis for significantly regulated genes in Tg4-42 mice compared to 5XFAD mice.

FIGURE 3.23 Validation of deep sequencing results through quantitative real-time polymerase chain reaction (qRT-PCR) analysis 


\section{List of Tables}

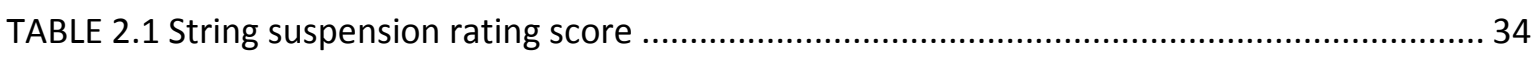

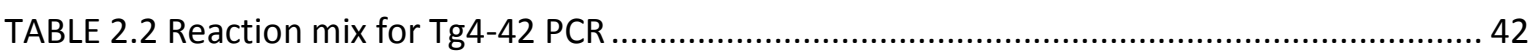

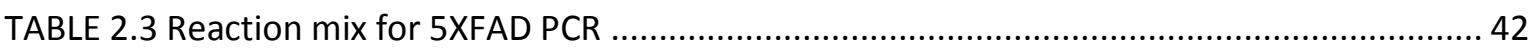

TABLE 2.4 PCR cycling program for genotyping Tg4-42 and 5XFAD mice ........................................ 43

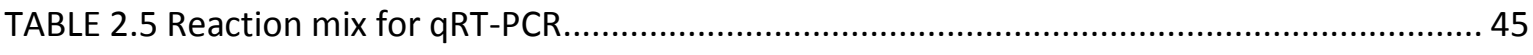

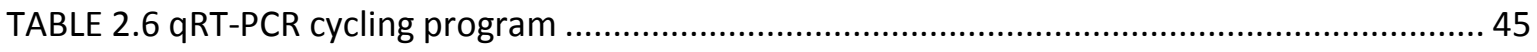

TABLE 2.7 List of primers used for mouse genotyping and qRT-PCR ............................................. 46

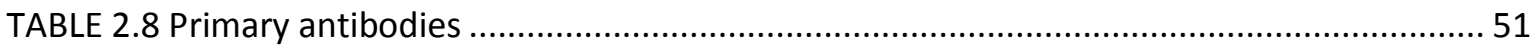

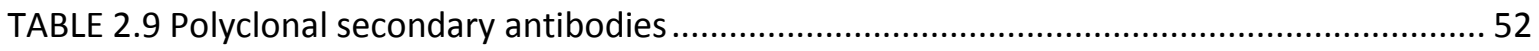

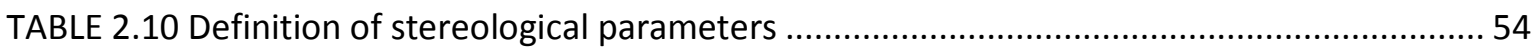

TABLE 2.11 Parameters for stereological analysis of neurons in the CA1 .................................... 55

TABLE 3.1 Number of exonic reads in brain tissue of wild-type and transgenic mice..................... 85

TABLE 3.2 Over-expressed transgenes in 5XFAD and Tg4-42 mice identified through RNASeq ..... 86

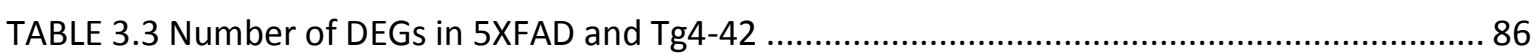

TABLE 3.4 List of differentially expressed transcripts in young 5XFAD mice .................................. 92

TABLE 3.5 List of transcripts exclusively expressed in aged Tg4-42 mice ........................................ 94

TABLE 3.6 List of transcripts differentially expressed in both aged Tg4-42 and 5XFAD mice.......... 96

TABLE 3.7 List of differentially expressed transcripts in aged 5XFAD mice ..................................... 99

TABLE 4.1 Comparison of the two transgenic mouse models 5XFAD and Tg4-42 ....................... 123 


\section{List of Abbreviations}

Please note that units described in the International System of Units (SI) and metric prefixes are not listed. Official gene symbols provided by the Mouse Genome Informatics resource (MGI) used in the text do not appear in this list as they are described in the text.

\begin{tabular}{|c|c|}
\hline Abbreviation & Description \\
\hline$A \beta$ & amyloid beta \\
\hline$A \beta_{\mathrm{pE3}-42}$ & amyloid beta 42 with a pyroglutamate modification at position 3 \\
\hline$A B C$ & avidin-biotin complex \\
\hline AD & Alzheimer's disease \\
\hline ADAM & a disintegrin and metalloproteinase \\
\hline AICD & amyloid precursor protein intracellular domain \\
\hline ALS & amyotrophic lateral sclerosis \\
\hline ANOVA & Analysis of variance \\
\hline APLP & APP-like protein \\
\hline ApoE & apolipoprotein E \\
\hline APP & amyloid precursor protein \\
\hline asf & area sampling fraction \\
\hline BACE1 & $\beta$-site cleaving enzyme 1 \\
\hline bp & base pair(s) \\
\hline CAMCOG & Cambridge Cognitive Examination \\
\hline cDNA & complementary DNA \\
\hline CDT & Clock Drawing Test \\
\hline CFC & contextual fear conditioning \\
\hline CNS & central nervous system \\
\hline CS & conditioned stimulus \\
\hline CSF & cerebral spinal fluid \\
\hline CT & computed tomography \\
\hline $\mathrm{DAB}$ & diaminobenzidine \\
\hline DAPI & 4'.6-diamidino-2-phenylindole \\
\hline DEG & differentially expressed gene \\
\hline $\mathrm{dd}_{2} \mathrm{O}$ & distilled, deionized water \\
\hline DNA & deoxyribonucleic acid \\
\hline DNase & deoxyribonuclease \\
\hline PBS & Dulbecco's Phosphate Buffered Salt Solution \\
\hline EC & entorhinal cortex \\
\hline EDTA & ethylenediaminetetraacetic acid \\
\hline EOAD & early-onset Alzheimer's disease \\
\hline ER & endoplasmic reticulum \\
\hline EST & expressed sequence tags \\
\hline $\mathrm{EtOH}$ & ethanol \\
\hline FAD & familial Alzheimer's disease \\
\hline
\end{tabular}




\begin{tabular}{|c|c|}
\hline FC & fear conditioning \\
\hline FCS & fetal cow serum \\
\hline GWAS & genome wide association studies \\
\hline hom & homozygous \\
\hline icv & intracerebroventricular \\
\hline IGAP & International Genomics of Alzheimer's Project \\
\hline IVC & individually ventilated cages \\
\hline $\mathrm{KI}$ & knockin \\
\hline LOAD & late-onset Alzheimer's disease \\
\hline LTP & long-term potentiation \\
\hline MANOVA & multivariate analysis of variance \\
\hline $\mathrm{MCl}$ & mild cognitive impairment \\
\hline MMSE & Mini-Mental State Examination \\
\hline MRI & magnetic resonance imaging \\
\hline MVB & multivesicular bodies \\
\hline MWM & Morris water maze \\
\hline $\mathrm{n}$ & number of biological replicates \\
\hline $\mathrm{N}$ & number of neurons \\
\hline NFT & neurofibrillary tangles \\
\hline NGS & next-generation sequencing \\
\hline NIA & National Institute on Aging \\
\hline NMDA & N-methyl-D-aspartate \\
\hline $\mathrm{P}$ & number of neurons \\
\hline PBS & phosphate buffered saline \\
\hline PCR & polymerase chain reaction \\
\hline PET & positron emission tomography \\
\hline PFA & paraformaldehyde \\
\hline pGlu & pyroglutamate \\
\hline PHF & paired helical filaments \\
\hline PSEN & presenilin \\
\hline Q & total markers counted \\
\hline QC & glutaminyl cyclase \\
\hline qRT-PCR & real-time quantitative PCR \\
\hline RNA & ribonucleic acid \\
\hline RNA-Seq & RNA-Sequencing \\
\hline rpm & revolutions per minute \\
\hline RT & Room temperature \\
\hline SAD & sporadic Alzheimer's disease \\
\hline SAGE & serial analysis of gene expression \\
\hline SDS & sodium dodecyl sulfate \\
\hline SEM & standard error of the mean \\
\hline SNP & single nucleotide polymorphism \\
\hline SPF & specific-pathogen-free \\
\hline ssf & section sampling fraction \\
\hline STZ & streptozotocin \\
\hline
\end{tabular}


TAE buffer

TBE buffer

TBS

TFC

TRH

tsf

US

WHO

WT

Z mean section thickness

Tris base/acetic acid buffer

Tris/borate/EDTA buffer

Tris-buffered saline

tone fear conditioning

thyrotropin releasing hormone

thickness sampling fraction

unconditioned stimulus

World Health Organization

wild-type

dissector height 


\section{INTRODUCTION}

1.1

Alzheimer's disease

In 1906 , at the $37^{\text {th }}$ meeting of the Society of Southwest German Psychiatrists in Tübingen, Alois Alzheimer presented the case of his patient Auguste Deter. In this single case, he described the main clinical and neuropathological characteristics of a disease that was later named after him: memory disturbance, neuron loss, miliary bodies (plaques) and dense bundles of fibrils (tangles) (Alzheimer, 1907; Alzheimer et al., 1995; Goedert, and Spillantini, 2006). Alois Alzheimer would likely not have imagined that today Alzheimer $(A D)$ is the most common neurodegenerative disease, with nearly 30 million cases worldwide (Alzheimer's Association, 2012).

\section{Clinical aspects of Alzheimer's disease}

\subsection{1}

Epidemiology

According to the World Health Organization (WHO) 35.6 million people worldwide were living with dementia in 2012. Due to an aging population this number is predicted to increase more than triple by the year 2050 (WHO, 2012). Currently, more than 1.4 million people are diagnosed with dementia in Germany (Deutsche Alzheimer Gesellschaft, 2014). Alzheimer's disease (AD) is the most common form of dementia and accounts for approximately 60 - $80 \%$ of the cases (Alzheimer's Association, 2012). To date, approximately 5.4 million Americans suffer from $A D$, which is the sixth leading cause of death in America. The health-care costs associated with $A D$ already add up to more than $\$ 200$ billion and are expected to increase to $\$ 1.1$ trillion in 2050 in the United States alone (Alzheimer's Association, 2012).

\subsubsection{Non-genetic risk factors}

The greatest and most obvious risk factor of $A D$ is aging (Blennow et al., 2006). Although with advancing age the risk for $A D$ increases dramatically, $A D$ is not part of the normal 
aging process. While one in eight people over the age of 65 years suffers from $A D$, nearly $45 \%$ of the over 85-year-olds have AD (Alzheimer's Association, 2012).

Epidemiological studies have suggested further risk factors for AD besides aging. Vascular diseases like atherosclerosis, hypercholesterolemia and coronary heart disease or heart failure elevate the risk of developing dementia (Kivipelto, 2001; Kivipelto et al., 2005; Qiu et al., 2006). There is also evidence that obesity, smoking and type II diabetes are associated with an increased risk for AD (Prince et al., 1994; Leibson et al., 1997; Kivipelto et al., 2005). Furthermore, several studies showed that head injury and traumatic brain injuries could be risk factors for AD (Plassman et al., 2000; McCullagh, 2001; Sivanandam and Thakur, 2012).

In contrast, several studies suggest that a healthy and physically active lifestyle, an antioxidant-rich Mediterranean diet and cognitive stimulating activities can reduce the risk of developing AD (Fratiglioni et al., 2004; Hall et al., 2009; Gu et al., 2010).

\subsubsection{Disease progression}

AD is a slowly progressive disease. The complex progression of neurodegeneration in AD patients results in memory impairment and decline in other cognitive abilities, often combined with non-cognitive symptoms like mood- and personality changes (Alzheimer's Association, 2012). Patients progress from mild and moderate $A D$ to severe $A D$ in the average time course of 7 to 10 years (Holtzman et al., 2011; Alzheimer's Association, 2012).

In 2011, the Alzheimer's Association and the National Institute on Aging (NIA) proposed new guidelines for the classification and diagnosis of $A D$ identifying three different stages of $A D$. These stages are preclinical $A D$, mild cognitive impairment $(\mathrm{MCl})$ due to AD and dementia due to AD. (Albert et al., 2011; Jack et al., 2011; McKhann et al., 2011; Sperling et al., 2011). During the preclinical phase, individuals do not present any measurable symptoms. However, it is estimated that changes in the brain and neurodegeneration already start 20 years before the initial clinical diagnosis of $A D$ (Blennow et al., 2006). Patients with $\mathrm{MCl}$ display a noticeable but mild cognitive decline compared to same-aged healthy individuals (Alzheimer's Association, 2012). Each year $10-15 \%$ of $\mathrm{MCl}$ cases convert into $\mathrm{AD}$ and in these cases $\mathrm{MCl}$ is an early stage of $\mathrm{AD}$ (Petersen, 2004; Visser et al., 2005). In the final stage, patients develop dementia due to $A D$. This stage is characterized among others through memory decline, language dysfunction, personality changes, motor impairments and visuo-spatial difficulties (Holtzman et al., 2011; Alzheimer's Association, 2012). At the terminal stage of the disease individuals are totally dependent on caregivers and unable to perform basic motor 
functions like swallowing. $A D$ is not only a progressive disease but ultimately fatal, often caused by pneumonia (Wada et al., 2001).

\subsubsection{Diagnosis and treatment}

\subsubsection{Diagnosis}

To date, a definite diagnosis of $A D$ can only be made post mortem by neuropathological examination. The criteria for the neuropathological confirmation of $A D$ are based on the analysis of amyloid plaques and neurofibrillary tangles (NFT) formation in the brain (McKhann, 1984; Braak and Braak, 1991).

However, cognitive and behavior changes as well as the family history of an individual can be used to make a probable diagnosis of AD prior to death (Alzheimer's Association, 2012). In the early stages of AD physical and multiple cognitive deficits are important requirements for an initial $\mathrm{MCl}$ diagnosis (Waldemar et al., 2007). For diagnosis patients must show, besides memory deficits, impairments in agnosia, aphasia, apraxia or executive functioning (organizing, etc.). These deficits must be gradual and severe enough to affect the abilities of an individual to carry out daily activities (American Psychiatric Association, 1995). A number of tests are available to evaluate the mental status of individuals over the course of the disease. Screening tests for AD include the Mini-Mental State Examination (MMSE), Clock Drawing Test (CDT), and Cambridge Cognitive Examination (CAMCOG) (Schmand et al., 2000; Aprahamian et al., 2010; Martinelli et al., 2014). Next to neuropsychological tests, neuroimaging methods play an important role in the diagnosis of $A D$ and the exclusion of alternative causes of dementia like brain tumours, hydrocephalus or subdural hematomas (Waldemar et al., 2007; Ballard et al., 2011). Magnetic resonance imaging (MRI), computed tomography (CT) and positron emission tomography with radiolabeled glucose (FDG-PET) or an amyloid-binding dye (PiB-PET) are used to diagnose $\mathrm{MCl}$ due to $A D$ and the transition into AD (Blennow et al., 2006; Perrin et al., 2009; Ballard et al., 2011). In addition, cerebral spinal fluid (CSF) biomarkers have been examined as a valuable diagnostic tool for $A D$ in several studies. Diagnosis of $M C l$ and $A D$ could be achieved through detection of reduced $A \beta_{x-42}$ as well as increased tau and phosphorylated tau levels (Mattsson et al., 2009; Perrin et al., 2009; Fiandaca et al., 2014).

\subsubsection{Treatment}

Currently, there is no cure for AD. Available medications only help to alleviate the symptoms and do not target the underlying molecular processes of the disease. There are 
two types of drugs available for AD: memantine and three different acetylcholinesterase inhibitors.

The cholinergic system is involved in memory and behavior (Bartus et al., 1982). According to the cholinergic hypothesis, a dysfunction of cholinergic neurons in AD leads to memory and cognitive impairments (Terry and Buccafusco, 2003). The routinely prescribed drugs donepezil, rivastigmine and galantamine are used to increase the availability of acetylcholine by inhibiting the catalytic function of acetylcholinesterase the enzyme that degrades acetylcholine in the synaptic cleft. There is evidence, that the acetylcholinesterase inhibitors can temporarily stabilize or even moderate the symptoms. However, all three drugs do not cure the disease or prevent its progression (Rogers, 1998; Bullock and Dengiz, 2005; Bullock et al., 2005; Wallin et al., 2011).

Memantine is an uncompetitive $\mathrm{N}$-methyl-D-aspartate (NMDA) receptor antagonist that is prescribed to patients with moderate to severe $A D$ (Tariot et al., 2004). In AD glutamate-mediated excitotoxicity is increased. Memantine is used to protect neurons from this excitotoxicity without disturbing the physiological function of NMDA receptors (Parsons et al., 2007). It is reported that memantine has a modest beneficial effect in patients with moderate to severe $A D$ and is suggested to be used in combination with cholinesterase inhibitors (Orgogozo et al., 2002; McShane et al., 2006; Peskind et al., 2006; Parsons et al., 2013).

\section{3}

\section{Neuropathological hallmarks of Alzheimer's disease}

\subsection{1}

\section{Amyloid plaques}

Amyloid plaques are one of the key neuropathological features in $A D$. The main proteinaceous components of these extracellular deposits are $A \beta$ peptides. $A \beta$ peptides are generated by enzymatic cleavage of the amyloid precursor protein (APP) (Holtzman et al., 2011; Serrano-Pozo et al., 2011). Amyloid plaques can be divided into two groups: diffuse and neuritic (Yamaguchi et al., 1988b; Holtzman et al., 2011).

Diffuse plaques (FIGURE $1.1 \mathrm{~B}$ ) consist of non-fibrilar depositions of $A \beta$ with nearly no detectable neuritic dystrophy. They vary in size from $50 \mu \mathrm{m}$ to several hundred $\mu \mathrm{m}$ (Yamaguchi et al., 1988a; Duyckaerts et al., 2009). Diffuse plaques are also found in the cortex of cognitive normal aged individuals (Serrano-Pozo et al., 2011).

Neuritic plaques (FIGURE 1.1 A) are one of the major hallmarks of $A D$ and can be detected with $\beta$-sheet staining dyes like Thioflavin-S and Congo Red (Serrano-Pozo et al., 2011). These extracellular $A \beta$ plaques consist of highly aggregated fibrillary $A \beta$ and are surrounded by swollen, degenerating axons and dendrites. In close proximity to neuritic 
plaques degenerated neurons, as well as astro- and microgliosis are observed (Holtzman et al., 2011; Selkoe, 2011). The density of amyloid fibrils varies as well as its size (10 to $120 \mu \mathrm{m})$ (Thomas and Fenech, 2007). Next to $A \beta$ other proteins including APP, tau and ubiquitin are found in neuritic plaques (Su et al., 1998; Duyckaerts et al., 2009). Plaque pathology commonly starts in the neocortex and later progresses to the hippocampus, basal ganglia and cerebellum (Serrano-Pozo et al., 2011). In the end stages of the disease, neuritic plaques are also found in the brainstem and other subcortical structures (Thal et al., 2002; Aldwin and Gilmer, 2013). However, the plaque load correlates poorly with the cognitive decline and severity of the disease (Billings et al., 2005; Schaeffer et al., 2011; Villemagne et al., 2011).

A

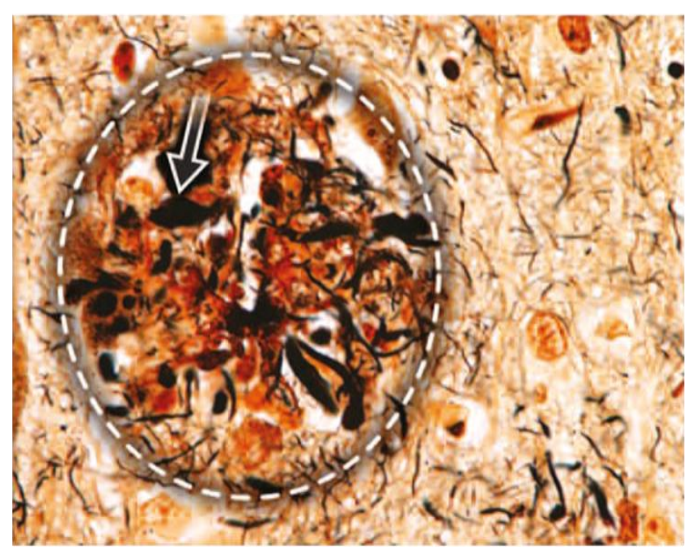

B

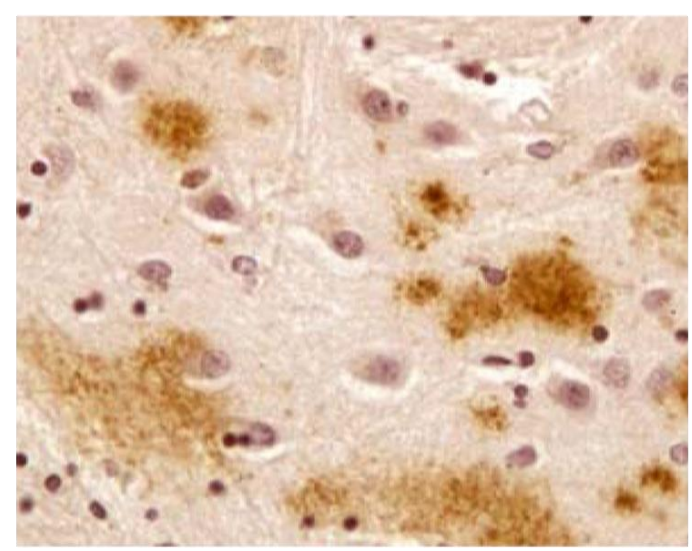

FIGURE 1.1 Amyloid plaques. (A) Photomicrograph of a neuritic amyloid plaque. Plaque is marked by a dashed circle. Arrow shows neurofibrillary tangle. Modified after Holtzman et al., 2011. (B) Diffuse A $\beta$ plaques. Modified after Duyckarts et al., 2009. Reprinted with permission of the copyright holder.

\subsection{2 \\ Neurofibrillary Tangles}

One of the neuropathological hallmarks of $A D$ are neurofibrillary tangels (NFTs) that were first described by Alois Alzheimer as 'intraneuronal filamentous inclusions' within the perikaryal region of pyramidal neurons (Alzheimer, 1907). Subsequently, ultrastructural studies revealed that the major component of NFTs are paired helical filaments (PHFs), which are mainly constituted of hyperphosphorylated tau (Goedert and Spillantini, 2006; Castellani et al., 2008).

Tau is a phosphoprotein that is abundant in neurons and produced in all nucleated cells (Duyckaerts et al., 2009; Galimberti and Scarpini, 2012). The normal function of tau is to bind to tubulin assembling and stabilizing microtubules (Goedert and Spillantini, 2006). However, in AD tau is abnormally hyperphosphorylated (FIGURE 1.2 A). The phosphorylation of tau reduces its microtuble binding activity and supports its self- 
aggregation forming PHFs in cell bodies and dystrophic neurites (Alonso et al., 1996; Holtzman et al., 2011).

The relevance and contribution of tau dysfunction to the pathogenesis of $A D$ remains unclear. It is well established that the tau pathology appear later in the progression of $A D$ than $A \beta$ deposition (Galimberti and Scarpini, 2012). However, neurofibrillary tangles, unlike the plaque pathology, correlate better with the severity of cognitive deficits (Holtzman et al., 2011).

\subsection{3}

Inflammation

Inflammatory processes are another pathological characteristic of AD (FIGURE 1.2 B). Microglia, astrocytes, the complement system as well as cytokines and chemokines are involved in the inflammatory reaction of the brain. Activated astrocytes and microglia are found in close proximity to neuritic plaques in $A D$, suggesting that $A \beta$ is a major trigger of glial activation (Itagaki et al., 1989; Pike et al., 1995a; Krause and Müller, 2010). Following activation, glial cells produce proinflammatory signal molecules including complement molecules as well as cytokines and chemokines (Tuppo and Arias, 2005; Rubio-Perez and Morillas-Ruiz, 2012)

A

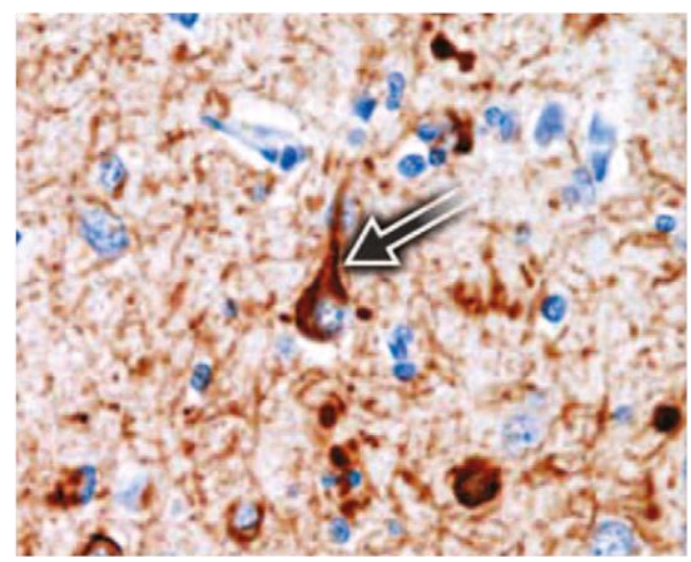

B

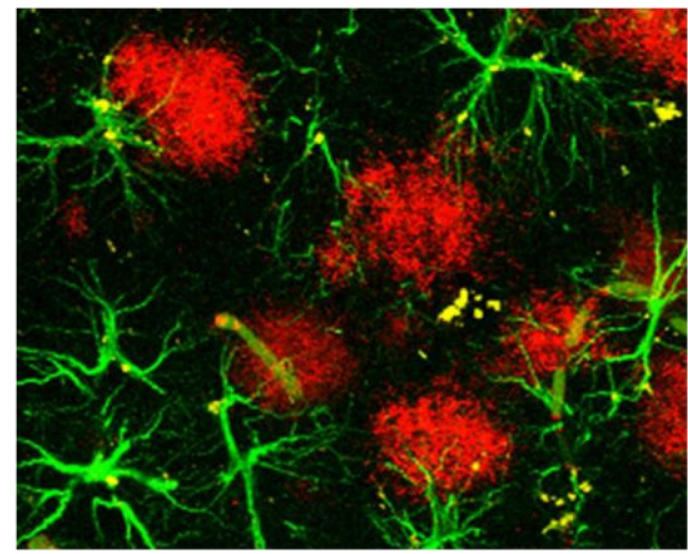

FIGURE 1.2 Tau and inflammation. (A) Hyperphosphorylated tau accumulation in neuronal cell bodies. Modified after Holtzman et al., 2011. (B) Confocal image of astrocyted labeled with GFAP (green) and plaques (red). Modified after Verkhratsky et al., 2010. Reprinted with permission of the copyright holder. 


\subsection{4}

Neuron loss

In addition to plaques and tangles, neuron loss is a main pathological hallmark of AD. Areas that are particular affected by neuron loss are the pyramidal layers of the hippocampus, the layer II of the entorhinal cortex, and some areas of the temporal, parietal and frontal neocortex (Holtzman et al., 2011; Serrano-Pozo et al., 2011). For example, stereology showed a significant neuron loss in the entorhinal cortex (EC) of patients with very mild AD (Gomez-Isla et al., 1996). While no neuron loss was observed in the CA1 in preclinical AD a profound neuron loss was reported in AD patients (West et al., 2004). Early studies suggested a correlation between the number of NFT in a region and the loss of neurons within the same region (Cras et al., 1995). Using unbiased stereology, it could be shown that the neuron loss in the superior temporal sulcus in fact partly correlates with the formation of NFT but exceeds it eminently. Strikingly, more than $50 \%$ of neurons in the superior temporal sulcus are lost in patients with AD (Gomez-Isla et al., 1997). Most recent reports suggest that intraneuronal or oligomeric $A \beta$ are instead crucial for cell death and neuron loss in AD (Bayer and Wirths, 2011; Larson and Lesné, 2012).

Cortical atrophy, that is mainly caused by neuron loss, is the most evident macroscopic characteristic of AD. Atrophy affects mainly the hippocampus, amygdale and entorhinal cortex and can be measured by MRI (Bottino et al., 2002). AD can be diagnosed with 80 to $90 \%$ accuracy through hippocampal atrophy measured by MRI (Jagust, 2006). Hippocampal atrophy also allows to predict the progression from $\mathrm{MCl}$ to $A D$ to a certain degree (Jack et al., 2005).

Synapse loss also contributes to the cortical atrophy of the AD brain. The number of lost synapses exceeds the decrease of neurons in the cortical area. Therefore, it can be assumed that synapse loss occurs before neuron loss (Serrano-Pozo et al., 2011). Synapse loss is an early indicator of the pathological processes in AD. It could be shown that patients with mild $A D$ have fewer synapses in the CA1 of the hippocampus than individuals with $\mathrm{MCl}$ or healthy controls (Scheff et al., 2007). Decreased synaptic density correlates directly with the severity of AD. Actually, synaptic density is a better correlate of cognitive decline than NFTs or neuron loss. (DeKosky and Scheff, 1990; Scheff et al., 1990; Scheff and Price, 1993; Ingelsson et al., 2004).

\section{$1.4 \quad$ The amyloid precursor protein}

The amyloid precursor protein (APP) is a highly conserved transmembrane, type-1, integral glycoprotein that is ubiquitously expressed and around $110-135 \mathrm{kDa}$ in size 
(Selkoe et al., 1988; Tharp and Sarkar, 2013). In human the APP gene is located on chromosome 21. APP is sequentially cleaved to generate $A \beta$, the main component of plaques (Korenberg et al., 1989).

Through alternative splicing 8 isoforms of APP are generated. Among these, APP695, APP751 and APP770 are the three major isoforms containing 695, 751, and 770 amino acids, respectively (Sandbrink et al., 1996). APP695 is mainly expressed in the CNS, particularly in neurons, while APP751 and APP751 are also widely expressed in other tissues (O'Brien and Wong, 2011). The highest levels of APP695 expression in the brain could be detected in the cerebellum, cortex and hippocampus (Sola et al., 1993).

APP belongs to a family of conserved type-I transmembrane proteins with the two mammalian homologs, APP-like protein 1 (APLP1) and 2 (APLP2) (Wasco et al., 1993; Slunt et al., 1994; Coulson et al., 2000). These proteins are processed in a similar way and have several common conserved motifs. However, the $A \beta$ domain in not conserved and unique to the APP protein (Bayer et al., 1999).

The physiological function of APP is still not understood. APP knockout mice are viable and fertile, indicating that APP does not play a role in vital functions or embryonic development. However, APP deficit mice show a moderate abnormal phenotype with cerebral gliosis, impaired learning and LTP, reduced body and brain size (Zheng et al., 1995; Dawson et al., 1999; Ring et al., 2007). The lack of a lethal phenotype in APP knockout mice may be attributed to the homologous amyloid precursor like proteins (APLPs) (Slunt et al., 1994; Heber et al., 2000). Although the physiological role of APP remains unclear, several function have been attributed to APP. These include neuronal protein trafficking along the axon, neurite outgrowth and synaptogenesis, transmembrane signal transduction, cell adhesion and calcium metabolism (Zheng and Koo, 2006).

APP can be processed via two major alternative pathways: the non-amyloidogenic and the amyloidogenic pathway (De-Paula et al., 2012).

In the non-amyloidogenic pathway (FIGURE 1.3), APP is cleaved by a $\alpha$-secretase releasing a large soluble $\mathrm{N}$-terminal fragment called SAPPa and a smaller C-terminal fragment (C83) (Esch et al., 1990; Sisodia et al., 1990). Three members of the disintegrin and metalloproteinase ADAM family have been suggested as the $\alpha$-secretase: the type-I transmembrane proteins ADAM-9, ADAM-10 and ADAM-17 (Asai et al., 2003). Recent studies indicate, that ADAM-10 acts as the main $\alpha$-secretase in neurons (Kuhn et al., 
2010). While sAPPa is released into the extracellular space, the 83-residue C-terminal fragment (C83) is membrane-bound. C83 is further cleaved by $\mathrm{Y}$-secretase into the soluble, $3 \mathrm{kDa}$ C-terminal fragment (p3) and the APP intracellular domain (AICD). The p3 peptide is rapidly degenerated (De-Paula et al., 2012; Sisodia, 1992; Zheng and Koo, 2011). While AICD is subjected to act as a transcriptional regulator, the function of $p 3$ is unknown (Chow et al., 2010). The cleavage of APP by $\alpha$-secretase occurs within the A $\beta$ domain, preventing the generation of amyloid peptides (Sisodia, 1992). In contrast to $A \beta$, several studies suggest that SAPPa is neuroprotective. SAPPa plays a role in neuronal plasticity, synaptogenesis, cell adhesion as well as early CNS development (Furukawa et al., 1996; Mattson, 1997; Chow et al., 2010). Interestingly, in APP deficient mice the expression of SAPPa was sufficient enough to rescue behavior and electrophysiological abnormalities suggesting that SAPPa accounts for most of the physiological functions of APP (Ring et al., 2007).

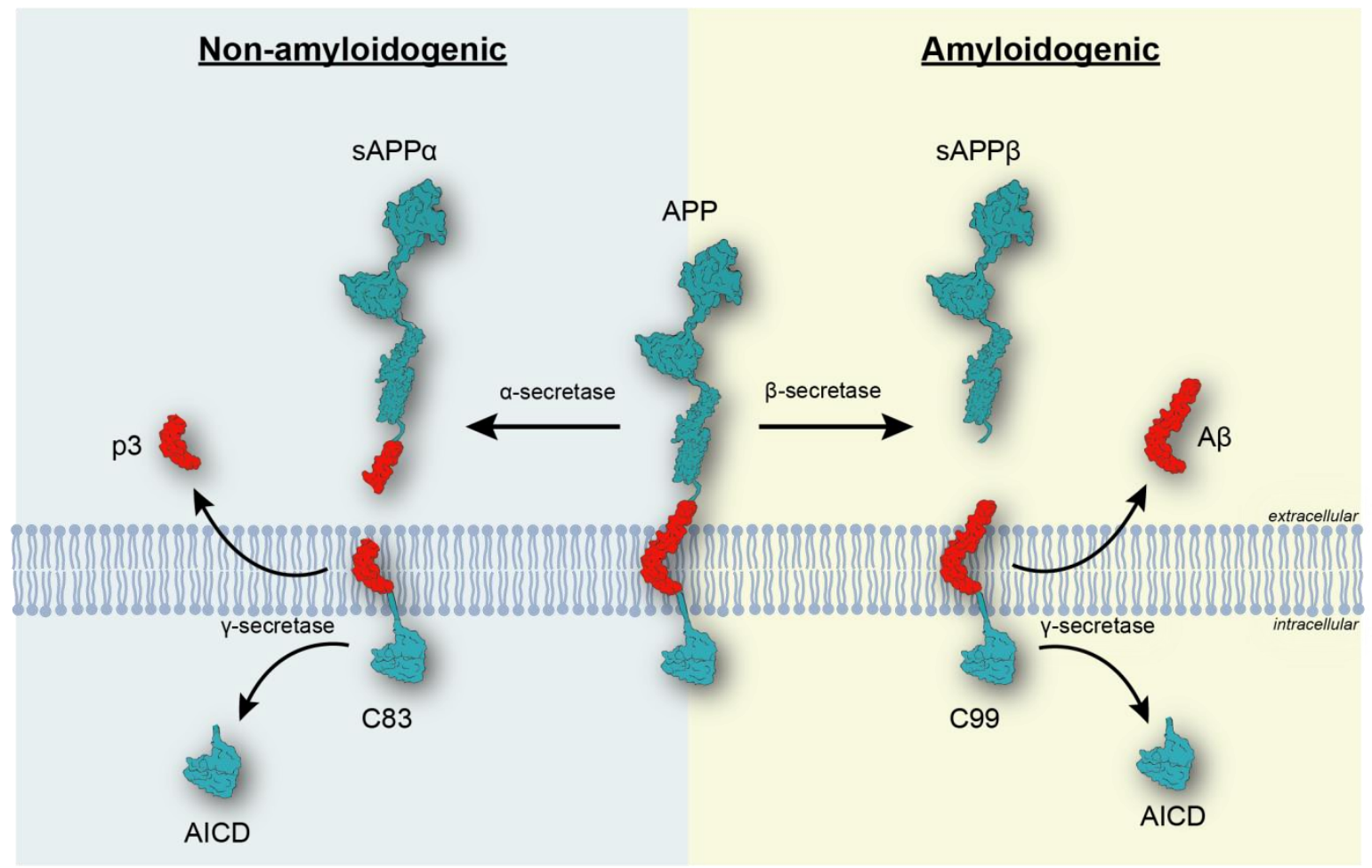

FIGURE 1.3 APP processing. Schematic diagram showing non-amyloidogenic and amyloidogenic APP processing. In the non-amyloidogenic processing APP is sequentially cleaved by $\alpha$-secretase and $\gamma$-secretase (left) releasing the $p 3$ fragment. In the amyloidogenic processing the $A \beta$ peptide is released via $\beta$-secretase and $\gamma$-secretase (right). 


\subsubsection{Amyloidogenic pathway}

In the amyloidogenic pathway (FIGURE 1.3), APP is cleaved by a $\beta$-secretase releasing a soluble $\mathrm{N}$-terminal fragment (SAPP $\beta$ ) and a longer C-terminal 99-residue fragment (C99). $\beta$-secretase cuts APP 16 amino acids upstream from the $\alpha$-secretase cleavage site. The resulting C99 fragment remains membrane associated and is subsequently cleaved within the transmembrane domain at different sites by $\gamma$-secretase generating $A \beta$ peptides of various lengths and AICD. $A \beta$ peptides rage from 37 to 43 amino acids in length (Citron et al., 1995; Wolfe, 2007; Zhang et al., 2011; De-Paula et al., 2012). Under normal physiological conditions $A \beta$ is mainly 40 amino acids long and the 42 amino-acid long $A \beta$, the main component of amyloid plaques, represent only a minor portion of the total $A \beta$ (Zhang et al., 2011).

The $\beta$-site cleaving enzyme 1 (BACE1) was identified by several groups as the primary $\beta$-secretase (Hussain et al., 1999; Sinha et al., 1999; Vassar et al., 1999; Yan et al., 1999). BACE1 is a membrane-bound aspartyl protease that is highly expressed in the brain, especially in regions affected by $A D$ including the cortex and the hippocampus (Vassar et al., 1999; Yang et al., 2003; Johnston et al., 2005). BACE1 is able to cleave A $\beta$ at two known positions: the aspartate at position 1 and the glutamate at position 11 (Sinha et al., 1999; Vassar et al., 1999). Next to BACE1, BACE2 (a homolog of BACE1) and cathepsin $B$ have been suggested as additional $\beta$-secretases. In several studies the inhibition of the protease cathepsin $B$ reduced $A \beta$ generation in vivo and in vitro. (Hook et al., 2005; Hook et al., 2009). However, the physiological contribution of these enzymes to APP cleavage requires further investigation.

The $\mathrm{y}$-secretase is a multiprotein complex of at least four components: presenilin 1 (PSEN-1) or presenilin 2 (PSEN-2), the type I transmembrane glycoprotein nicastrin (Nct) and the two multipass transmembrane proteins anterior pharynx-defective-1 (Aph-1) and presenilin enhancer-2 (Pen-2) (O'Brien and Wong, 2011). Y-secretase also cleaves a variety of other type I integralmembrane proteins including the Notch receptor, cadherin, tyrosinase and CD44 (Wolfe, 2010; Strooper et al., 2012).

\section{5}

\section{The amyloid cascade hypothesis}

The amyloid cascade hypothesis has been proposed more than 20 years ago by Hardy and Higgins (1991) to explain the etiology and pathogenesis of AD. According to the amyloid cascade hypothesis the deposition of $A \beta$ is the causative event of $A D$ pathology leading to NFTs, neuron loss, vascular damage and cognitive deficits (FIGURE 1.4). The hypothesis claims that increased $A \beta$ production or decreased $A \beta$ clearance leads to 
accumulation of hydrophobic $A \beta_{40}$ and $A \beta_{42}$ and the formation of insoluble extracellular plaques. Plaques then trigger a cascade of mischievous changes eventually resulting in synapse loss, neuron loss, brain atrophy and dementia (Hardy and Allsop, 1991; Pimplikar, 2009). Several observations support this theory. Genetic studies of familial AD cases are probably the strongest arguments supporting the amyloid hypothesis. Inherited mutations in APP, PSEN-1 and PSEN-2 alter the A levels and lead to increased depositions of $A \beta$ in plaques (Pimplikar, 2009; Bertram et al., 2010). In addition, many AD mouse models harboring familial $A D$ mutations develop an age-dependant pathology similar to the one seen in $A D$ patients including $A \beta$ plaques, gliosis and memory deficits (Duyckaerts et al., 2008; Pimplikar, 2009). Down syndrome patients exhibit a triplication of the APP gene as it is located on chromosome 21. The triplication leads to an AD pathology with amyloid plaques and NFTs in these patients (Rumble et al., 1989; Schupf, 2002). Furthermore, apolipoprotein $E \varepsilon 4$ allele (ApoE\&4), the most important risk factor for late-onset $A D$ (LOAD), is associated with increased $A \beta$ deposition and reduced $A \beta$ clearance (see section 1.9) (Bickeboller et al., 1997; Castellano et al., 2011). In contrast, mutations in tau lead to tauopathies like frontotemporal dementia instead of $A D$, suggesting that NFTs are not responsible for initiating AD and rather occur subsequent to $A \beta$ aggregation (Hutton et al., 1998; Goedert and Jakes, 2005; Iqbal et al., 2005a).

However, the amyloid cascade hypothesis has several major flaws. First of all, the plaque load does not correlate well with the severity of dementia. Furthermore, a robust plaque pathology could also be observed in otherwise cognitive normal individuals who show no signs of dementia (Snowdon, 2003; Villemagne et al., 2008; Pimplikar, 2009). Moreover, in many $A D$ mouse models memory impairment and pathological changes occur long before the appearance of plaques (Walsh and Selkoe, 2007; Lesne et al., 2008). Additionally, in an anti-A $\beta$ active vaccine trial (AN1792) patients showed no cognitive improvements although a massively reduced plaque pathology could be observed (Holmes et al., 2008).

\subsubsection{Intraneuronal amyloid hypothesis}

Growing evidence points to intraneuronal $A \beta$ as a key contributor in $A D$ pathology. The first observations of intracellular $A \beta$ in neurons were made nearly 30 years ago by Masters et al. (1985). It was later shown that intraneuronal $A \beta$ accumulates in $A D$ vulnerable brain regions including pyramidal neurons of the hippocampus and entorhinal cortex. Intracellular $A \beta$ depositions were detected prior to the development of NFTs and extracellular plaques (Gouras et al., 2000; Fernandez-Vizarra et al., 2004). Similar observations were made in patients with Down syndrome as young patients lacking 
plaques showed intracellular $A \beta$ (Gyure et al., 2001; Mori et al., 2002). Furthermore, decreased intraneuronal $A \beta$ is accompanied by increased plaque pathology and may therefore have a role in the development of extracellular plaques (Gyure et al., 2001; Gouras et al., 2010). The appearance of intracellular $A \beta$ prior to PHF and plaques indicates that it represents one of the earliest pathological events in the AD brain (Wirths and Bayer, 2012).

Several animal models reinforced the important role of intracellular $A \beta$ in $A D$ by showing a correlation between intracellular $A \beta$ and neuron loss (Casas et al., 2004; Christensen et al., 2008; Jawhar et al., 2010). In vitro studies in cultured human neurons also showed accumulation of intracellular $A \beta$ (Skovronsky et al., 1998; Takahashi et al., 2004). Increased $A \beta_{42}$ levels and an elevated $A \beta_{42} / A \beta_{40}$ ratio was shown in neurons from sporadic and familial $A D$ cases using laser capture microdissection of pyramidal neurons in AD brains (Aoki et al., 2008).

Intraneuronal $A \beta$ can origin from two distinct sources. In addition to intracellular cleavage of APP, the reuptake from the extracellular space represents the second source of intraneuronal $A \beta$ (Wirths et al., 2004). Several receptors have been proposed to facilitate the reuptake of $A \beta$, including $A p o E$ receptors, nicotinic acetylcholine ( $n A C h R$ ) receptors, integrins and $\mathrm{N}$-methyl-D-aspartate (NMDA) receptors as well as advanced glycation end product receptors. $A \beta_{42}$ is degraded poorly and its accumulation inside neurons has a variety of severe consequences (Mohamed and Posse de Chaves, 2011). Primary neuronal cell culture experiments showed intraneuronal $A \beta$ localization in endosomes, lysosomes and multivesicular bodies (MVBs) (Runz et al., 2002; Takahashi et al., 2004; Almeida et al., 2006). Accumulation of $A \beta$ in lysosomes leads to loss of lysosomal impermeability and leakage of lysosomal contents like proteases and cathepsins resulting in apoptosis and necrosis (Ditaranto et al., 2001; Nixon, 2005; Liu et al., 2010). Due to its high quantity of membranes and low $\mathrm{pH}$, neuronal MVBs are a good location for $A \beta$ aggregation. Accumulation of $A \beta$ in MVBs can impair their trafficking and degradative function (Takahashi et al., 2002; Mohamed and Posse de Chaves, 2011). Furthermore, a correlation between intracellular $A \beta$ and apoptotic markers was observerd in AD patients as well as AD mouse models (Chui et al., 2001; Mohamed and Posse de Chaves, 2011). Taken together, the findings from AD and DS patients, mouse models and in vitro studies emphasized the role of intracellular $A \beta$ as a potential key player in the development and progression of $A D$. Based on these perceptions a modified $A \beta$ hypothesis was formulated: the intraneuronal amyloid hypothesis (FIGURE 1.4). 
B-Amyloid Cascade

Extracellular $A \beta 40 / 42 \uparrow$

Extracellular $\mathrm{A} \beta 40 / 42$ plaques

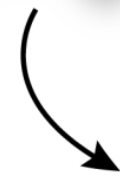

Modified $\beta$-Amyloid Cascade

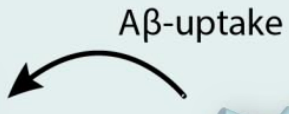

Plaque formation

Intraneuronal $A \beta 40 / 42 \uparrow$

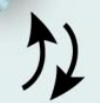

Soluble extracellular

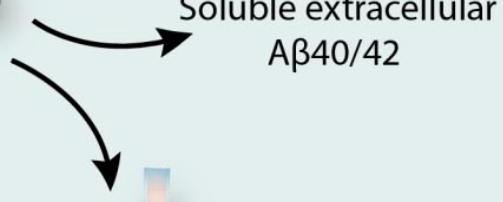

Accumulation of intraneuronal $A \beta 40 / 42$

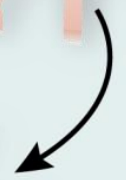

Dysfunction of synapse and neuron

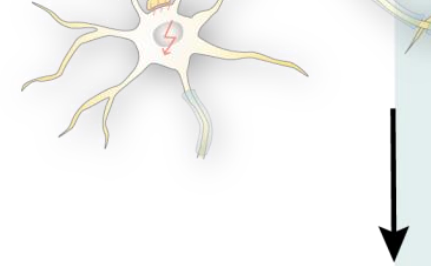

Synapse and neuron loss

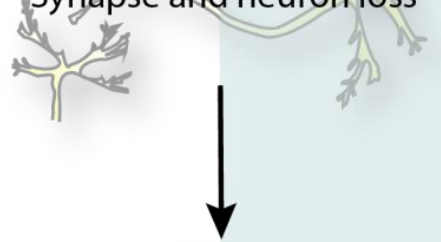

Cerebral atrophy

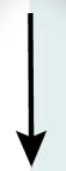

Dementia

FIGURE 1.4 Amyloid hypothesis. Schematic diagram illustrating the amyloid cascade hypothesis (left) and the modified intraneuronal $A \beta$ hypothesis (right). 
Soluble $A \beta$ monomers have a molecular weight of $4 \mathrm{kDa}$ and a high tendency to polymerize and aggregate. The formation of plaque-forming, insoluble $A \beta$ fibrils from these monomers is complex. Monomeric $A \beta$ can self-associate to form dimers, trimers or higher-order aggregates larger than $100 \mathrm{kDa}$ (Benilova et al., 2012). The majority of $A \beta$ appears as lower oligomers from dimers to octamers. Soluble oligomers are non-fibrillar structures that remain stable in aqueous buffers even after high speed centrifugation (Walsh and Selkoe, 2007).

Monomers are assumed to be in equilibrium with different oligomeric forms. However, when a certain threshold is reached, a polymerization process into paranuclei is initiated. These paranucleis self-associate into protofibrils and ultimately fibrils, the major component of plaques. Alternatively, protofibrils can dissociate again into lower-order species (Walsh and Selkoe, 2007; Roychaudhuri et al., 2009). The aggregation of $A \beta$ can be divided in two kinetic phases. $A \beta$ monomers accumulate slowly to form oligomers during the 'lag phase'. In the 'elongation phase', oligomeric nuclei promote protofibrils formation and create mature amyloid fibrils (FIGURE 1.5) (Kumar et al., 2011).

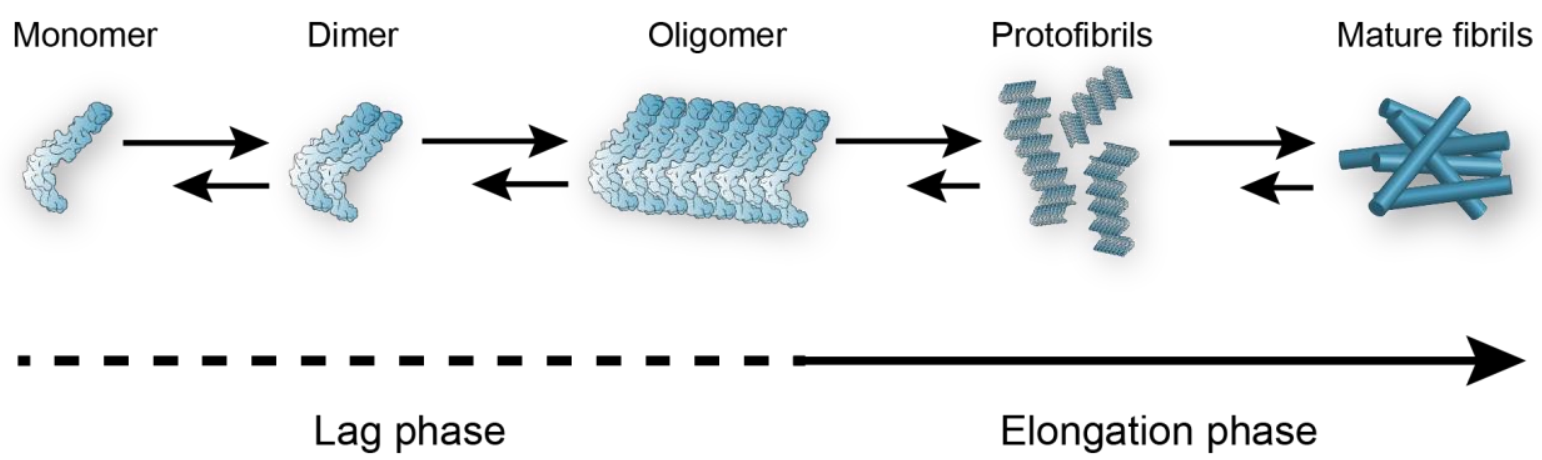

FIGURE 1.5 Schematic diagram of $A \beta$ aggregation. $A \beta$ monomers accumulate slowly to form oligomers during the 'lag phase' (dashed line). In the 'elongation phase' (straight line), oligomeric nuclei promote protofibril formation. Protofibrils aggregate creating mature amyloid fibrils. Figure created after Kumar et al., 2011.

Increased formation and aggregation of neurotoxic $A \beta$ oligomers have been reported to be crucially involved in the initiation and progression of AD (Kayed et al., 2003; Haass and Selkoe, 2007; Li et al., 2009). Further, it has been demonstrated that soluble oligomeric $A \beta_{42}$ rather than plaques correlates best with the synaptic loss and cognitive decline in $A D$ patients (McLean et al., 1999; Bayer and Wirths, 2010). Oligomers are able to disrupt synaptic plasticity and inhibit hippocampal LTP (Walsh et al., 2000). Due to its amphiphilic 
nature, $A \beta$ aggregates and forms higher oligomers in vitro (Pimplikar, 2009). These oligomers could also be isolated from AD brain extracts and AD mouse models (Lesne et al., 2008; Shankar et al., 2008).

\section{$1.7 \quad$ Amyloid beta variants}

\subsection{1}

\section{Truncated amyloid beta species}

$A \beta$ peptides are a heterogeneous set of peptides. Two major $A \beta$ forms are 40 and respectively 42 residues long. It was shown that $A \beta_{40}$ is the major component of cerebral vascular amyloid deposits, while $A \beta_{42}$ is the main component of neuritc plaques (Iwatsubo et al., 1994; Suzuki et al., 1994). $A \beta_{42}$ species are more hydrophobic and have a higher tendency to aggregate than $A \beta_{40}$. Furthermore, $A \beta_{42}$ is more neurotoxic and crucial for plaque formation (Jarrett et al., 1993; Jan et al., 2008). Next to $A \beta_{1-40}$ and $A \beta_{1-42}$ a variety of $C$-terminal truncated $A \beta$ forms have been reported including $A \beta_{1-15 / 16}$ and $A \beta_{1-37 / 38 / 39}$ (Wiltfang et al., 2002; Portelius et al., 2012). In vitro studies identified a number of longer $A \beta$ variants like $A \beta_{43}, A \beta_{45}, A \beta_{46}$ and $A \beta_{48}$ in cell lines. Longer $A \beta$ variants have also been identified in AD mouse models (Esh et al., 2005; Van Vickle, G D et al., 2008; Welander et al., 2009). In sporadic and familial $A D$ cases $A \beta_{43}$ was detected and even more prevalent than $A \beta_{40}$ in plaque cores (Welander et al., 2009).

Several $\mathrm{N}$-terminal truncated $A \beta$ variants have been reported in $A D$. Instead of aspartate that is normally found at the first position of $A \beta$, these $A \beta$ forms possess a variety of different N-termini (Masters et al., 1985; Selkoe et al., 1986; Mori et al., 1992; Sergeant et al., 2003). In vitro experiments showed that shortening of the $\mathrm{N}$-terminus enhances $A \beta$ aggregation (Pike et al., 1995b). Several proteases have been proposed to be involved in the generation of $\mathrm{N}$-truncated $A \beta$ species (FIGURE 1.6). However, the exact enzymatic activities are in most cases not fully understood (Bayer and Wirths, 2014). BACE1 is the major protease responsible for the generation of $A \beta_{1-x}$ in $A D$. In addition, BACE1 is also able to cleave $A \beta$ between Tyr-10 and Glu-11, leading to the release of $A \beta_{11-x}$ peptides (Vassar et al., 1999). $A \beta_{2-x}$ species have been detected to be elevated in brains and decreased in the CSF of AD patients. It has been proposed that these peptides arise from a combination of BACE1 followed by aminopeptidase A (Wiltfang et al., 2001; Bibl et al., 2012). Recently, it was demonstrated that the metalloprotease meprin $\beta$ may also be involved in the generation of $A \beta_{2-x}$ species (Bien et al., 2012). $A \beta_{3-x}$ has been observed in plaques in LOAD patients and AD mouse models (Casas et al., 2004; Guntert et al., 2006). Another important protease involved in $\mathrm{N}$-truncated $\mathrm{A} \beta$ generation is the zinc-metalloprotease neutral endopeptidase or neprilysin 
(NEP). NEP cleaves $A \beta$ peptides between Arg-2 and Glu-3, Glu-3 and Phe-4 or Arg-5 and His-6 while leaving full-length APP intact (Howell et al., 1995; Iwata et al., 2001; Bayer and Wirths, 2014). Furthermore, the myelin basic protein has been reported to cleave $A \beta$ between Phe- 4 and Arg- 5 in the N-terminus of the $A \beta$-sequence (Liao et al., 2009). $A \beta_{4-x}$ species (section 1.7.1.1) are one the first $A \beta$ discovered and very abundant in $A D$ and vascular dementia (Masters et al., 1985; Lewis et al., 2006), while $A \beta_{5-x}$ was detected in AD patients and non-demented controls (Sergeant et al., 2003; Murayama et al., 2007). Except for the generation of $A \beta_{11-x}$, the largest known amino-truncations occur before the amino acids Asp-7, Ser-8 and Gly-9. Angiotensin-converting enzyme has been proposed as an enzyme involved in the generation of $A \beta_{8-x}$ (Kalback et al., 2002; Sergeant et al., 2003; Kummer and Heneka, 2014). Taken together, $N$-truncated $A \beta$ variants are the main constituent of amyloid plaques in $A D$ and can also be detected intraneuronal (Gouras et al., 2000; Bayer and Wirths, 2014).

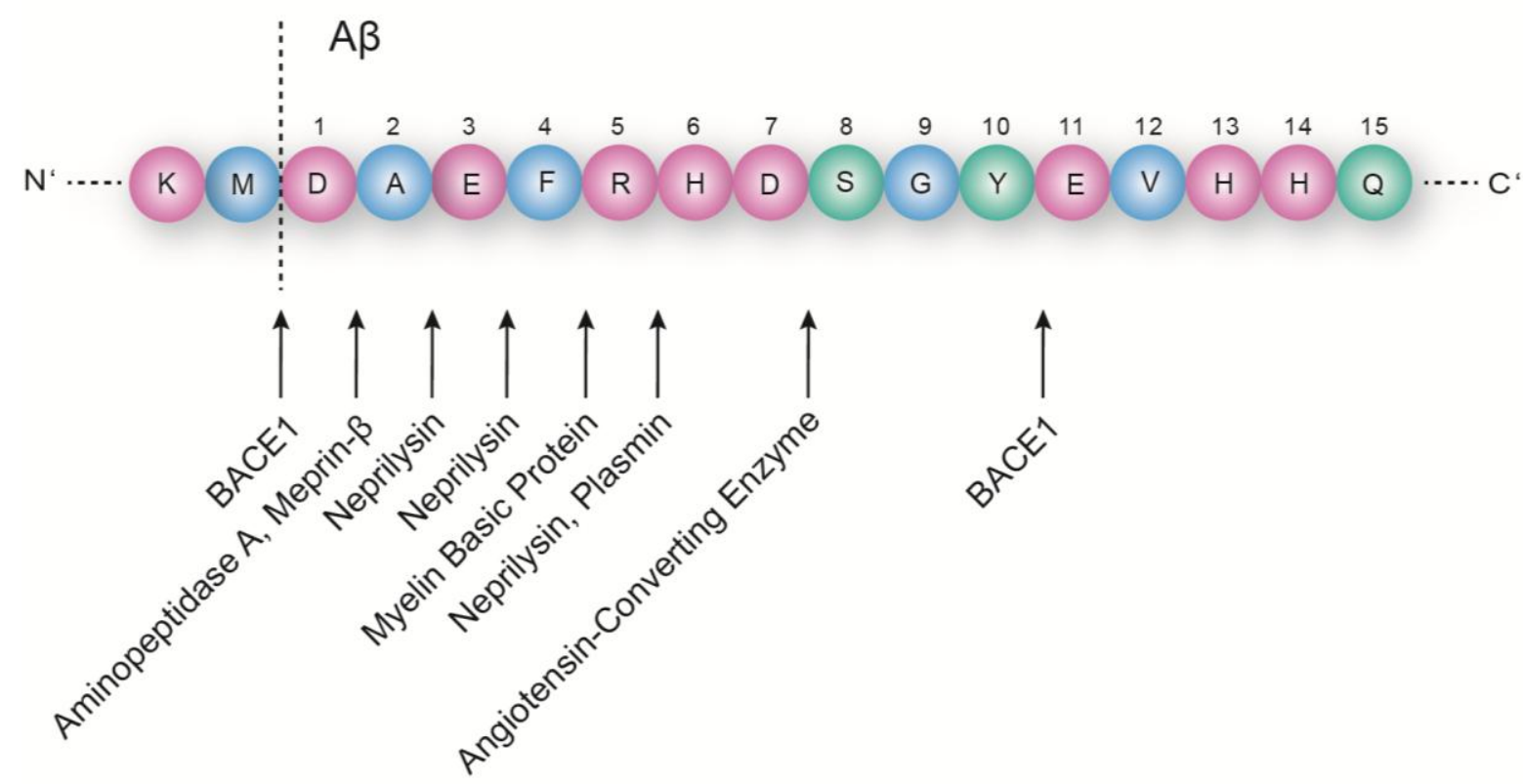

FIGURE 1.6 Schematic overview of proteases and cleavage sites involved in generation of $\mathbf{N}$-truncated $\mathbf{A} \boldsymbol{\beta}$. Sequence of the first 15 amino acids of the $\mathrm{N}$-terminus of human $A \beta$ presented in the one-letter-code. Color code for amino acids: pink indicates uncharged polar side-chains, green uncharged polar side-chains and blue hydrophobic non-polar side-chains. Figure created after Bayer and Wirths, 2014. 


\subsubsection{Amyloid beta 4-42}

$A \beta_{4-42}$ is very abundant in $A D$ and was discovered as early as 1985 (Masters et al., 1985). However, relatively little is known about the contribution of $A \beta_{4-42}$ to the development and progression of AD. Masters et al. (1985) identified $64 \%$ of $A \beta$ peptides started with a Phe4 in plaque cores of two sporadic $A D$ cases. Furthermore, this $\mathrm{N}$-truncated $A \beta$ variant beginning with phenylalanine was found relatively prevalent in $A D$, vascular dementia patients and aged controls (Lewis et al., 2006). While the exact amount of $A \beta_{4-42}$ is not clear, a recent study, using immunoprecipitation and mass spectroscopy, showed that $A \beta_{4-42}$ is one of the major fractions in the cortex and hippocampus of $A D$ patients (Portelius et al., 2010). $A \beta_{4-42}$ has also been identified in amyloid deposits from familial Danish dementia and vascular dementia patients (Tomidokoro et al., 2005; Lewis et al., 2006). Furthermore, $A \beta_{4-42}$ was detected in cotton wool plaques in FAD patients with a V261I PSEN-1 mutation (Miravalle et al., 2005). It is proposed that the degrading enzymes neprilysin is responsible for the cleavage of $A \beta_{1-42}$ (FIGURE 1.6) and the generation of $A \beta_{4-42}$ (Howell et al., 1995; Iwata et al., 2001; Carson and Turner, 2002).

Sedimentation studies indicate that $A \beta_{4-42}$ aggregates faster than longer $\mathrm{N}$-termini $A \beta$ variants (Pike et al., 1995b). Moreover, the $\mathrm{N}$-terminus specifies fibrillization behavior (Haupt et al., 2012). Recently, it was demonstrated through short-term treatment of primary cortical neurons, that $A \beta_{4-42}$ is as toxic as pyroglutamate $A \beta_{3-42}$ and $A \beta_{1-42}$. Furthermore, treatment of wild-type mice using intraventricular $A \beta$ injection with $A \beta_{4-42}$ induced significant working memory deficits. These deficits were similar to the ones induced through pyroglutamate $A \beta_{3-42}$ and $A \beta_{1-42}$ (Bouter et al., 2013).

\subsubsection{Modified amyloid beta species}

Various post-translational modifications of $A \beta$ have been described (Kummer and Heneka, 2014). Besides pyroglutamylation, phosphorylation and oxidation, these modifications include nitration, racemization, isomerization and glycosylation (FIGURE 1.7). Oxidation and nitration of $A \beta$ are induced in response to the inflammatory milieu in $A D$ (Heneka et al., 2010). Increased oxidative stress has been observed in $\mathrm{MCl}$ and $\mathrm{AD}$ brains. The oxidation of methionine at position 35 is the most abundant $A \beta$ oxidation (Naslund et al., 1994; Kummer and Heneka, 2014). Oxidation of $A \beta$ has been described to prevent the formation of protofibrils (Hou et al., 2002).

Phosphorylation can occur at three different sites in the $A \beta$ peptide. Phosphorylation of serine at position 8 increases the formation of oligomeric $A \beta$ aggregates (Kumar et al., 2011; Kummer and Heneka, 2014). Racemization of aspartate 
and serine residues were detected in $A D$ patients and could be induced by radicals in vitro (Shapira et al., 1988; Tomiyama et al., 1994; Tambo et al., 2013). Furthermore, peptides are vulnerable to spontaneous isomerization especially at asparagine and aspartate. The formation of isoaspartate effects the secondary structure and influence the aggregation properties of $A \beta$ (Szendrei et al., 1994; Kummer and Heneka, 2014).

Probably the most important modification of $A \beta$ is the formation of pyroglutamate (pGlu)-modified amyloid peptides. After removal of the first two amino acids, presumedly through aminopeptidase A, glutamate is converted to pyroglutamate in a dehydration reaction. In addition, it was proposed that spontanous $\mathrm{Cu}^{2+}$-mediated amide hydrolysis may be resposible for the removal of the fist two residues from $A \beta_{1-x}$ (Drew et al., 2010). The pGlu formation is then catalyzed by glutaminyl cyclase (QC), that is incread in $A D$ patients (Schilling et al., 2004; Schilling et al., 2008). Besides $A \beta_{p E 33}$, pyroglutamate modifications at $A s p-11$ were discovered $\left(A \beta_{p E 11}\right)$ that seem to emerge through alternative BACE1 cleavage in APP (Liu et al., 2002). pGlu modifications lead to an increased aggregation propensity, disturbed proteolytical degradation and increased toxicity (Pike et al., 1995b; Kummer and Heneka, 2014). It has been shown that $A \beta_{p E 3}$ oligomerizes more quickly and forms fibrillar, $\beta$-sheet structures more rapidly than $A \beta_{1-x}$ in vitro (He and Barrow, 1999; Harigaya et al., 2000; Schlenzig et al., 2009). $A \beta_{p E 3}$ is a major $A \beta$ species in $A D$ and a main component of plaques. Saido et al. (1995) showed that $A \beta_{p E 3}$ was found in an equal or greater amount in senile plaques than $A \beta_{1-x}$. They further suggested that $A \beta_{p E 3}$ deposition precede that of other $A \beta$ species (Saido et al., 1995).
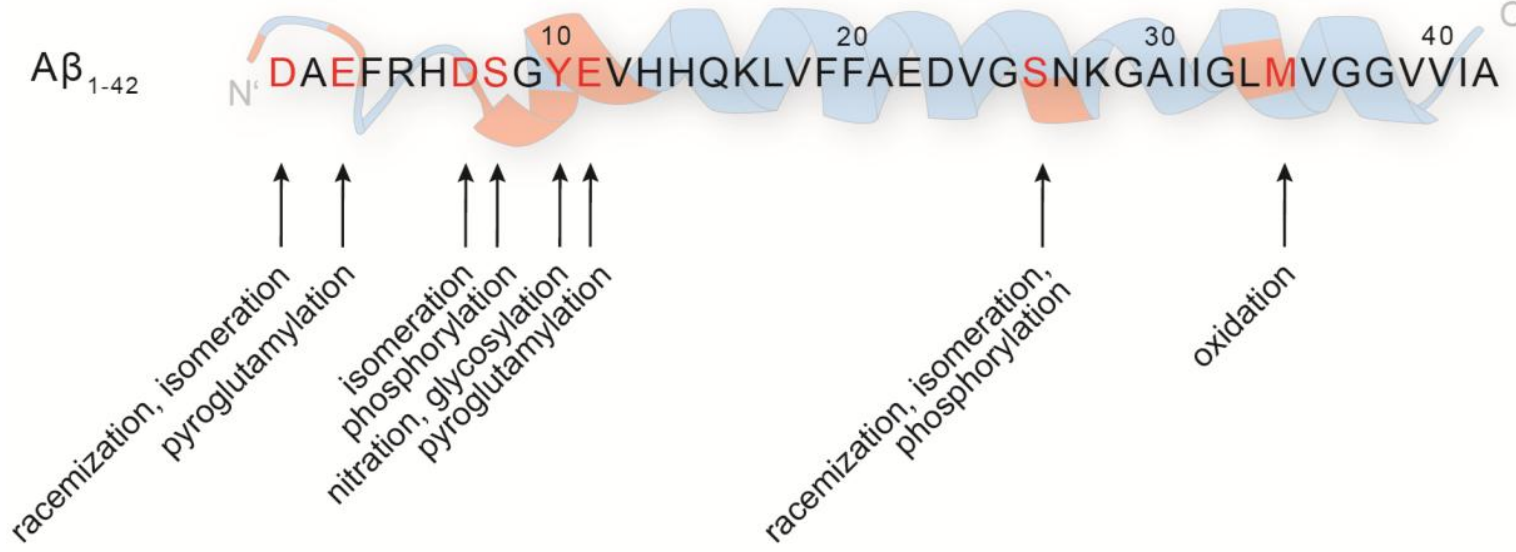

FIGURE 1.7 Schematic overview of post-translational modifications in $A \beta_{1-42}$. Sequence of the amino acids of human $A \beta_{1-42}$. Red letters indicate amino acids that are subjected to posttranslational modifications. Amino acids are represented in the one-letter code. Figure created after Kummer and Heneka, 2014. 
The detection of mutations in the APP and PSEN genes in patients with familial AD led to the generation of a variety of transgenic animal models. A wide range of transgenic mouse models have been developed expressing mutant human APP. The first AD transgenic mouse model based on mutant APP was the PDAPP model. These mice express human APP with the Indiana mutation (V717F) under the platelet derived growth factor promoter. PDAPP mice develop an age-dependant extracellular plaque pathology in addition to dystrophic neurites, astrocytosis and microgliosis. (Games et al., 1995; Reilly et al., 2003). Moreover, they show progressive synaptic loss and develop age-dependant memory deficits without neuron loss (Irizarry et al., 1997; Chen et al., 2000; Dodart et al., 2000). The Tg2576 mouse also possesses a single APP mutation and is one of the most widely studied AD models. Human APP containing the Swedish FAD mutation (K670N/M671L) is overexpressed in these mice under the control of the hamster prion promoter. Tg2576 mice develop an age-dependant plaque pathology along with dystrophic neuritis and gliosis similar to PDAPP mice. A significant plaque pathology in Tg2576 mice can be detected between 11 and 13 months. Strikingly, these mice develop working and spatial reference memory deficits prior to a significant plaque pathology (Hsiao et al., 1996; Holcomb et al., 1998; Elder et al., 2010). Subsequently, several other $A D$ mouse models were generated with human APP containing one or more FAD mutations. Generally speaking, these mice show elevated $A \beta$ levels and extracellular $A \beta$. Furthermore, many of these mice also show signs of inflammation and develop behavior and cognitive deficits (Sturchler-Pierrat et al., 1997; Chishti et al., 2001; Games et al., 2006; Elder et al., 2010).

Next to APP transgenic mouse models, many PSEN mice have been developed. Transgenic mice expressing mutated human PSEN consistently show elevated $A \beta_{42}$ levels without developing a plaque pathology (Cavanaugh et al., 2014). The lack of plaques may be due to lower levels of $A \beta_{42}$ in the PSEN lines in comparison to APP models. It could also be explained through the different solubility of human and murine $A \beta$ (Jankowsky et al., 2007). However, a number of PSEN models develop altered axonal transport, impaired calcium homeostasis in the ER and mild cognitive deficits (Duyckaerts et al., 2008).

In order to create a more aggressive $A D$ pathology mouse models with a combination of multiple APP mutations or APP and PSEN mutations were created. Double transgenic mice expressing mutated PSEN and APP develop plaques earlier than single transgenic APP mice and show increased inflammation and behavior deficits (Duff et al., 1996; Holcomb et al., 1998; Casas et al., 2004; Cavanaugh et al., 2014). Although these 
mouse models show a wide spectrum of $A D$ pathology, they lack some key feature of $A D$ including NFT formation. To overcome the lack of NFTs in AD mouse models, FAD and tau mutations from frontotemperal dementia have been combined. The $3 \times \mathrm{Tg}$ model is an example for a triple transgenic mouse model. These mice, generated by injecting two transgenes containing APP-Swedish and P301L mutations into a PS1M146V knock-in mice, show next to plaque depositions, a NFT pathology (Oddo et al., 2003). Furthermore, most $A D$ mouse models do not exhibit the significant neuron loss seen in $A D$ patients. Neuron loss could be mainly detected in mice with a combination of APP and PSEN mutations. APP751SL/PS1M146L, APP/PS1K1 and 5XFAD mice are some examples for AD models with neuron loss (Casas et al., 2004; Schmitz et al., 2004; Oakley et al., 2006; Jawhar et al., 2010).

The use of transgenic mouse models has led to new insights regarding the role of intraneuronal $A \beta$ accumulation in $A D$. Intraneuronal $A \beta$ has been reported in variety of mouse models including Tg2576, 3xTg, APP/PS1K1 and 5XFAD (Casas et al., 2004; Billings et al., 2005; Oakley et al., 2006; Takahashi et al., 2013). Furthermore, several mouse models showed a link between significant neuron loss and intraneuronal $A \beta$ accumulation (Wirths and Bayer, 2012).

Familial $A D$ accounts for a minor fraction of all $A D$ cases, however all of the previously described $A D$ mouse models rely on one or more FAD mutations. Rodents do not develop a spontaneously AD pathology as they age (Sarasa and Pesini, 2009). To date, there has been nearly no success in the generation of sporadic AD mouse models. A rodent model that shows at least some aspects of SAD has been generated by intracerebroventricular (icv) injection of streptozotocin (STZ). These mice are generated based on the detection of an insulin-resistant brain state in SAD. STZ is injected to induce such a brain insulin resistant state. These icv-STZ mice show memory and learning deficits, decreased energy metabolism, neuroinflammation, altered synaptic proteins and increased hyperphosphorylated tau in the brain (Salkovic-Petrisic et al., 2006; de la Monte, Suzanne M. and Wands, 2008; Chen et al., 2012; Chen et al., 2013).

Next to mice, a variety of other animals have been proposed to study AD including dogs, rats, rabbits, primates and even dolphins. However, mice remain the most commonly used vertebrates to study $A D$ especially due to their relatively short reproductive cycle, their small size, large litters and easy handling (Sarasa and Pesini, 2009). Several non-vertebrates, like the Caenorhabditis elegans and Drosophila melanogaster have also be used and genetically manipulated to study certain aspects of AD (Wu and Luo, 2005; Prüßing et al., 2013). AD mouse models, as well as other animal models, have highly contributed to the understanding of the pathology in AD. However, 
none of the animal model mimics all human $A D$ features, they rather reflect various parts of the disease and therefore provide insights in different aspects of $A D$.

\subsection{1}

\section{The 5XFAD mouse model}

The 5XFAD mouse model, first described by Oakley et al. (2006), is a double transgenic APP/PS1 mouse model that coexpresses five familial Alzheimer's disease (FAD) mutations. 5XFAD mice overexpress the 695 amino acids isoform of human APP carrying the Florida (1716V), London (V717I) and Swedish (K670N/M671L) mutations, together with mutant PSEN-1 (M146L; L286V) under the control of the murine Thy-1 promoter (FIGURE 1.8). Mice showed stable germline transmission and coinheritance of the APP and PSEN-1 transgenes over multiple generations. Therefore, mice breed as if they are a single transgenic mouse model (Oakley et al., 2006; Eimer and Vassar, 2013). In order to facilitate a better comparison with other $A D$ mouse models 5XFAD mice, that were originally generated as a C57/B6xSJL strain, were backcrossed onto a C57BL/B6 background (Jawhar et al., 2010).

The Florida, London and the two PSEN mutations result in an increased production of $A \beta_{x-42}$, while the Swedish mutation increases the levels of total $A \beta$. Plaque pathology in these mice starts between two and three months of age and is accompanied by astrocytosis and microgliosis. These features increase massively with age (Oakley et al., 2006). Extracellular plaques have also been detected in the spinal cords of three months old 5XFAD mice (Jawhar et al., 2010). In addition to extracellular plaques, 5XFAD exhibit intracellular $A \beta$ in the cortical Layer 5 and the subiculum starting at 1.5 months of age (Eimer and Vassar, 2013). Intraneuronal $A \beta$ precedes plaque formation and correlates well with neuron loss in these brain regions. The 5XFAD mouse is one of a few mouse models exhibiting significant neuron loss. Unbiased stereology revealed a significant neuron loss in the pyramidal neurons of the Layer 5 cortex and the subiculum starting at 9 months of age (Eimer and Vassar, 2013). Strikingly, the CA1 region of the hippocampus lacking intraneuronal $A \beta$ shows amyloid plaques but no neuron loss (Jawhar et al., 2010).

Furthermore, 5XFAD develop age-dependant synaptic degeneration indicated by the reduction of synaptic markers including synaptophysin, syntaxin, and postsynaptic density protein 95 (Oakley et al., 2006). Swollen presynaptic terminals and axonal processes develop intraneuronal BACE1 and $A \beta$ prior to plaque formation. Swelling and dystrophic changes in these structures are associated with deposition of extracellular $A \beta$ plaques (Zhang et al., 2009). 
5XFAD mice develop age-dependant progressive behavior deficits. Working memory, spatial memory and episodic memory deficits have been described in these mice as well as decreased anxiety. They show among others impairments in the Y-maze, Morris mater maze, the conditioned taste aversion task and contextual fear conditioning (Ohno et al., 2006; Devi and Ohno, 2010; Jawhar et al., 2010; Devi and Ohno, 2013). Next to cognitive impairments, 5XFAD mice also show a decline in motor function. The motor phenotype correlates well with extensive spinal cord pathology, including agedependent axonal degeneration and intraneuronal $A \beta$ accumulation (Jawhar et al., 2010).

A

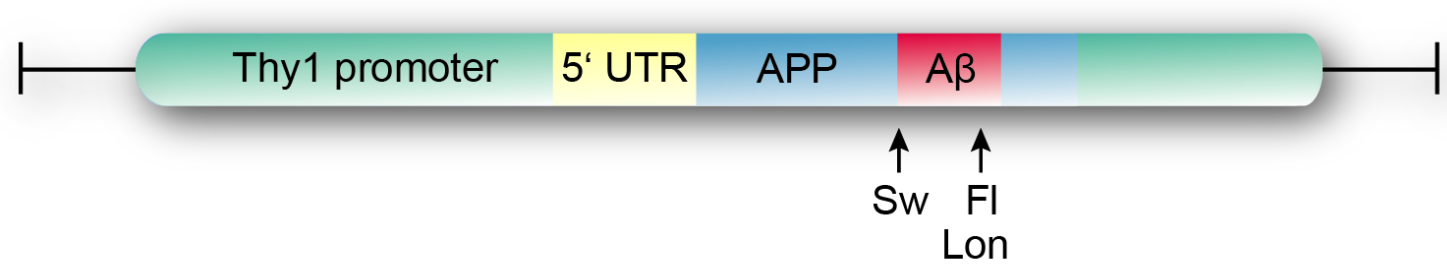

B

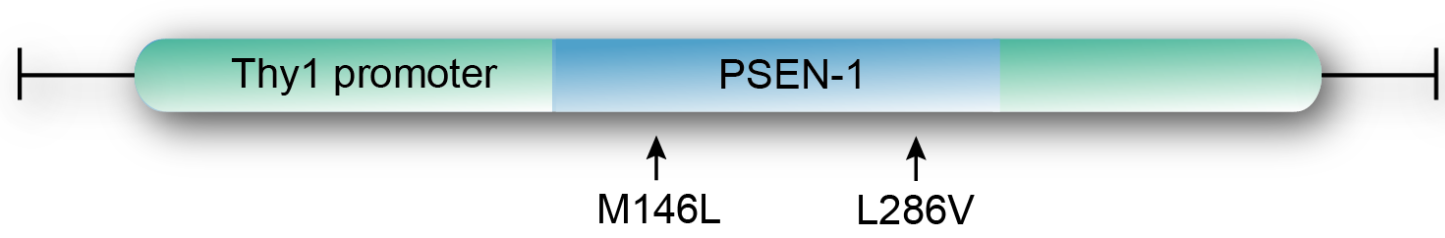

FIGURE 1.8 The 5XFAD transgenes. Schematic diagram of (A) Thy-1 APP and (B) Thy-1 PSEN-1 transgenes in the 5XFAD mouse model. FAD mutations are indicated by arrows. Abbreviations: SW = Swedish mutation; Lon = London mutation; Fl = FL mutation. Figure created after Oakley et al., 2006.

Based on the age of onset, $A D$ can be divided into early (EOAD) and late-onset $A D$ (LOAD). Both forms of AD have a genetic component (Bettens et al., 2013). While EOAD and LOAD cannot be clinical distinguished, EOAD usually shows a more rapid progression than LOAD (Reitz and Mayeux, 2014).

EOAD accounts for 5 - $10 \%$ of all AD cases and patients are typical younger than 65 years old when the disease manifests. Nearly half the patients with EOAD have at least one first-degree relative that is also affected by the disease. The autosomal-inherited form of EOAD is the so called familial form of AD (FAD) (Campion et al., 1999). Only a subgroup of patients displays this early-onset familial form of $A D$ that is caused by rare 
single mutations in either the amyloid-protein-precursor (APP) or the presenilin 1 (PSEN1) and presenilin 2 (PSEN-2) genes. To date, more than 160 mutations in these three genes, encoding for proteins involved in APP processing and A $\beta$ generation, have been described (Bertram and Tanzi, 2005; Blennow et al., 2006; Bettens et al., 2013).

APP was the first gene associated with EOAD and the responsible mutations are located in and near the $A \beta$ sequence. The majority of these mutations are heterozygous missense mutations in or around amyloid- $\beta$-coding exons 16 and 17 (Goate et al., 1991; Bettens et al., 2013). So far, 32 missense mutation have been identified in the APP gene (Ryan and Rossor, 2010). Moreover, whole-gene duplications, recessive missense mutations and small deletions have been described (Rogaev et al., 1995; Sleegers et al., 2006; Di Fede et al., 2009; Kasuga et al., 2009; Thonberg et al., 2011). Most APP mutations occur in the vicinity of the secretase cleavage sites and thereby affecting the APP cleavage and altering the overall $A \beta$ levels or the $A \beta_{42} / A \beta_{40}$ ratio (Holtzman et al., 2011). The first APP missense mutation was discovered in a British kindred and therefore named London mutation (Goate et al., 1991). This mutation (APP717) near the $Y$-secretase sites alters secretase cleavage leading to an increased $A \beta_{42} / A \beta_{40}$ ratio without effecting the total $A \beta$ production (Suzuki et al., 1994). The Florida mutation (APP716) increases the $A \beta_{42}$ levels in a similar way (Eckman et al., 1997; Jonghe et al., 2001). In contrast, the Swedish mutation (KM670/671NL) near the $\beta$-secretase cleavage site increases the production of all $A \beta$ species (Citron et al., 1992; Mullan et al., 1992). Furthermore, mutations also occur within the $A \beta$ sequence $C$-terminal to the $\alpha$-secretase cleavage site. These mutations can modify the properties of $A \beta$ leading to increased aggregation and oligomer/protofibril generation as well as a less effective $A \beta$ clearance (Grabowski et al., 2001; Nilsberth et al., 2001; Van Nostrand et al., 2001; Stenh et al., 2002).

Most mutations are reported in the PSEN-1 gene and these mutations account for the majority of $A D$ cases with an onset even prior the age of 50 . Mutations in the PSEN-1 and PSEN-2 gene result in an impairment of the $\mathrm{Y}$-secretase mediated cleavage of APP. The partial loss of function of the $\gamma$-secretase leads to an increased $A \beta_{42} / A \beta_{40}$ ratio (Wolfe, 2007). The shift is caused by an increased production of $A \beta_{42}$ and/or decreased production of $A \beta_{40}$ (Scheuner et al., 1996; Holtzman et al., 2011).

The majority of $A D$ cases occur after the age of 65 (LOAD). The vast majority of $A D$ patients displays no known mutations and therefore suffers from the sporadic lateonset form of AD (SAD) (Blennow et al., 2006). Although the exact causes of LOAD are unknown, it is most likely caused by a combination of several genetic and environmental factors. Twin studies prognosticated a $80 \%$ heritability of LOAD forms (Gatz et al., 2006). To date, the apolipoprotein $\mathrm{E}(\mathrm{ApoE}) \varepsilon 4$ allele is the only known certain genetic risk factor 
for sporadic AD (Blennow et al., 2006; Selwood et al., 2009). The ApoE gene is located on chromosome 19q13 and there are three common alleles of ApoE in humans. The variants $\varepsilon 2$, $\varepsilon 3$ and $\varepsilon 4$ only differ from each other by one amino acid at position 112 or 158 of the protein. While the $\varepsilon 4$ variant is a genetic risk factor for LOAD, the $\varepsilon 2$ allele is protective against LOAD (Corder et al., 1993; Holtzman et al., 2011). Individuals with one ApoE $\varepsilon 4$ allele have a three times higher risk of developing $A D$ than non-carriers. For homozygous $\varepsilon 4$ carriers the risk increases even 15-fold (Bickeboller et al., 1997). However, not all $\varepsilon 4$ carriers develop $A D$ and $\varepsilon 4$ is neither sufficient nor necessary for developing LOAD (Reitz and Mayeux, 2014). ApoE is a lipid-binding protein that is highly expressed in the CNS. (Strittmatter et al., 1993) While its precise function is not clear, ApoE plays a role in neuronal plasticity, cholesterol transport and inflammation. It is suspected that the ApoE\&4 leads to decreased clearance and/or increased $A \beta$ aggregation as well as cholesterol homeostasis (Bertram and Tanzi, 2005; Kim et al., 2009). However, a variety of additional genetic loci have been proposed to be involved with LOAD (Bertram and Tanzi, 2001).

\subsubsection{From genome-wide association studies to next-generation sequencing}

Commercially available microarray chips containing more than 1.2 million single nucleotide polymorphism (SNPs) initiated the era of genome wide association studies (GWAS) (Sherva and Farrer, 2011). Since 2009, several large GWAS were performed to search for genes that are involved in $A D$. These studies revealed 20 novel potential loci for LOAD and suggested new pathways that may be involved in AD. The first studies identified CLU (clusterin), PICALM (phosphatidylinositol-binding clathrin assembly protein), CR1 (Complement receptor type 1) and BIN1 (amphiphysin II) as susceptibility loci (Harold et al., 2009; Lambert et al., 2009; Seshadri et al., 2010). Clusterin is a lipoprotein that is involved in lipid transport and $A \beta$ aggregation. CR1 is a cell surface receptor that is part of the complement system. It can bind $A \beta$ oligomers and therefore may play a role in A $\beta$ clearance (Tosto and Reitz, 2013). These initial studies recognized loci mainly associated with the following pathways: immune response, APP processing, lipid metabolism, and endocytosis (Reitz and Mayeux, 2014). The second set of GWAS identified five additional susceptibility loci $(A B C A 7, C D 2 A P, C D 33, E P H A 1$ and $M S 4 A 4 A / M S 4 A 4 E / M S 4 A 6 E$ cluster) that are all presumably involved in the immune system (Hollingworth et al., 2011; Naj et al., 2011). In addition, ABCA7 is also involved in APP processing and lipid metabolism (Kaminski et al., 2000; Kielar et al., 2003). The most recent and largest GWAS by the International Genomics of Alzheimer's Project (IGAP) reported 11 new susceptibility loci for AD (CASS4, CELF1, FERMT2, HLA- 
DRB5/HLA-DRB1, INPP5D, MEF2C, NME8, PTK2B, SLC24A4/RIN3, SORL1 and ZCWPW1). Besides ApoE, the study confirmed eight of the nine previously described candidate-genes, with the exception being CD33. The associated loci reinforced pathways identified by the earlier GWAS and highlighted additional pathways including hippocampal synaptic function and axonal transport (Lambert et al., 2013). However, all new detected loci have only a small effect size with odds ratio ranging from 1.1 to 1.5 and low-risk factors for AD. Therefore, ApoE is still by far the strongest risk factor for LOAD (Tosto and Reitz, 2013).

It seems that the limit to the number of GWAS detectible variants is reached, while a large percentage of the heritability of $A D$ is still unknown. Next-generation sequencing (NGS) may be able to contribute to the remaining 'missing heritability' and identify rare variants that are not yet captured (Sherva and Farrer, 2011; Medway and Morgan, 2014). NGS allows affordable and fast massive-scale DNA sequencing. The principle behind all NGS methods is to randomly fragment DNA into smaller pieces to create a library. Libraries are then sequenced at a high coverage in millions of parallel reactions (Metzker, 2010; Berglund et al., 2011). The identified strings of bases, called reads, are mapped into the reference genome of the species. In the absence of a reference genome reads can also be assembled de novo (Magalhães et al., 2010).

NGS has not only the potential to detect new risk factors in large samples, it can also be beneficial in small patient groups. The technique can give new insights into families with an unexplained AD cause as well as in groups of unrelated cases. NGS has the potential to identify rare variants with moderate effects through assessment of the entire exomes or genomes of individuals. Furthermore, NGS can be applied for resequencing the top loci revealed by GWAS (Bettens et al., 2013; Medway and Morgan, 2014; Sharma et al., 2014). The triggering receptor on myeloid cells 2 (TREM2) gene was the first LOAD susceptibility locus identified via NGS approach (Medway and Morgan, 2014). Two groups independently reported variants of TREM2 gene as a new risk factor for LOAD in white populations (Guerreiro et al., 2013; Jonsson et al., 2013). TREM2 has been associated with immune response and inflammation and is involved in phagocytosis of apoptotic neurons (Takahashi et al., 2005; Hsieh et al., 2009; Slattery et al., 2014). It could be shown that NGS whole-exome sequencing is capable of identifying not only very rare mendelian causes of $A D$ but also low-frequency variabilities with medium-effect sizes (Jin et al., 2012). This example highlights that NGS has the potential to play a crucial role in the identification of new risk factors for AD especially LOAD. 


\section{9 .2}

Transcriptome profiling: RNA-Sequencing

The transcriptome reflects the cellular activity as it shows the genes that are expressed within a tissue at any given time point. Northern blots, reverse-transcription PCR, expressed sequence tags (ESTs) and serial analysis of gene expression (SAGE) made it possible to analyze RNA expression (Krumlauf, 1994; Velculescu et al., 1995; Ginzinger, 2002; Milla et al., 2002). However, only the development of microarrays allowed rapid large-scale studies of gene expression. Microarrays are able to simultaneously analyze large numbers of transcripts while relying on previous knowledge about the genome sequence. Several studies using transcriptome microarray analyses were performed over the last years to identify genes that are differentially expressed and therefore may be involved in the pathogenesis of AD (George et al., 2010).

The recent developments in NGS offer a more comprehensive and most of all unbiased approach for transcriptome analysis. NGS is not restricted to sequencing DNA and can be used in a similar fashion to perform transcriptome sequencing (RNA-Seq). In addition to mRNA, non-coding RNA, microRNA and whole RNA can be sequenced (Eminaga et al., 2013; Handel et al., 2013). This enables to investigate changes in gene expression and to look at post-transcriptional modifications, mutations and SNPs as well as alternative gene spliced transcripts. Importantly, unlike microarrays, RNA-Seq is not limited to the detection of transcripts that match with existing genomic sequences (Wang et al., 2009). NGS can be used to compare the gene expression profiles between different cell types or genotypes. It can access the absolute transcript levels of already sequenced as well as unsequenced organisms (Mutz et al., 2013). Furthermore, multiple studies already indicate that next-generation sequencing is more useful and particularly suitable to investigate the pathogenesis of complex neurodegenerative diseases like $A D$ (Twine et al., 2011). For example, Sultan et al. (2008) claimed that deep sequencing of nonribosomal RNA can detect up to $25 \%$ more genes than microarrays analyses. The quantification of transcript isoforms is also a strength of RNA-Seq (Malone and Oliver, 2011). Variances in gene expression might contribute to the initiation or progression of AD. Especially alternative splicing might play an important role in LOAD (Twine et al., 2011). RNA-Seq facilitates direct access to reads spanning exon/exon boundaries. That way NGS enables studying the expression of different isoforms (Malone and Oliver, 2011). 
The aim of this thesis was to investigate the role of $A \beta_{4-42}$ in the etiology of $A D$. $\mathrm{N}$-truncated $A \beta_{4-42}$ is highly abundant in the brain of $A D$ patients (Portelius et al., 2010). However, a possible role in AD etiology has largely been neglected.

The thesis is divided in two parts (FIGURE 1.9). The aim of the first project was to study the effects of chronic exposure of $A \beta_{4-42}$ in vivo. Therefore the recently generated mouse model Tg4-42 that over-expresses $A \beta_{4-42}$ was used (Bouter et al., 2013). Next to heterozygous Tg4-42 mice a new homozygous Tg4-42 line was generated (Tg4-42 ${ }_{\text {hom }}$ ). Characterization of 3-, 8- and 12-month-old heterozygous mice was performed. Moreover, homozygous mice were assessed with three and eight months of age. Using immunohistochemistry the brain pathology was examined in these mice. Furthermore, a battery of motor and cognitive tasks was conducted to assess behavioral performance in Tg4-42 and Tg4-42 hom mice. Moreover, using unbiased stereology the number of neurons in the CA1 region of the hippocampus was determined in heterozygous and homozygous mice at different time points. In addition, the new monoclonal antibody NT4X-167 that recognizes the $\mathrm{N}$-terminus of $\mathrm{N}$-truncated $A \beta$ was generated. NT4X-167 was used to assess $A \beta_{4-x}$ pathology in Tg4-42 and $5 X F A D$ mice via immunohistochemistry. In summary, the main aim of the first part of the thesis was to provide a detailed behavioral neuropathological, and stereological analysis of $\mathrm{Tg} 4-42$ and $\mathrm{Tg} 4-42_{\text {hom }}$ in an agedependant manner.

In the second part of the thesis, a comparative gene expression analysis of brain tissue of two different $A D$ mouse models using next-generation sequencing was performed. The transcriptome includes all RNA transcripts expressed in a given tissue and renders a profile of genes that are expressed at the studied time point. Altered gene expression profiles may therefore provide information about the genes and mechanisms involved in the molecular pathogenesis of diseases like AD. Technical approaches using transcriptome microarray analyses were performed over the last years to identify genes that are differentially expressed and therefore may be involved in the pathophysiology of AD (George et al., 2010). The recent developments in next-generation sequencing (deep sequencing) offer a more comprehensive and most of all unbiased approach for transcriptome analysis. Multiple studies already indicate that next-generation sequencing is more useful and particularly suitable to investigate the pathogenesis of complex neurodegenerative diseases like AD (Twine et al., 2011). The Tg4-42 mouse model was compared with the well-established, plaque-developing 5XFAD mouse model (Oakley et al., 2006). Brain transcriptomes of young and old Tg4-42, 5XFAD and WT control mice were analyzed by deep-sequencing of non-ribosomal RNA (RNA-Seq). Differential 
expression analysis, comparing transgenic mice with age-matched WT mice was performed. The aim of this part was to elucidate the similarities and distinctions in expression profiles of Tg4-42 and 5XFAD mice. Furthermore, the behavior of these two mouse models was compared using the fear conditioning and Morris water maze task.

\section{Objectives of Project I:}

- Generate a homozygous Tg4-42 mouse line

- Develop a novel antibody, which recognizes the $\mathrm{N}$-terminus of $\mathrm{N}$-truncated $A \beta$

- Examine $A \beta_{4-x}$ pathology in 5XFAD and Tg4-42 mice using the novel antibody NT4X-167

- Examine the neuropathology resulting from $A \beta_{4-42}$ expression in Tg4-42 and Tg4-42 ${ }_{\text {hom }}$ mice

- Determine if age-dependent motor or memory changes occur in Tg4-42 and Tg4-42 ${ }_{\text {hom }}$ mice

- Investigate if $A \beta_{4-42}$ pathology causes neuron loss

\section{Objectives of Project II:}

- Compare the behavior deficits of Tg4-42 and 5XFAD mice

- Perform deep-sequencing of non-ribosomal RNA on 5XFAD, Tg4-42 and WT mice

- Verify differentially expressed genes by qRT-PCR

- Analyze the similarities and distinctions in expression profiles of 5XFAD and Tg4-42 mice 


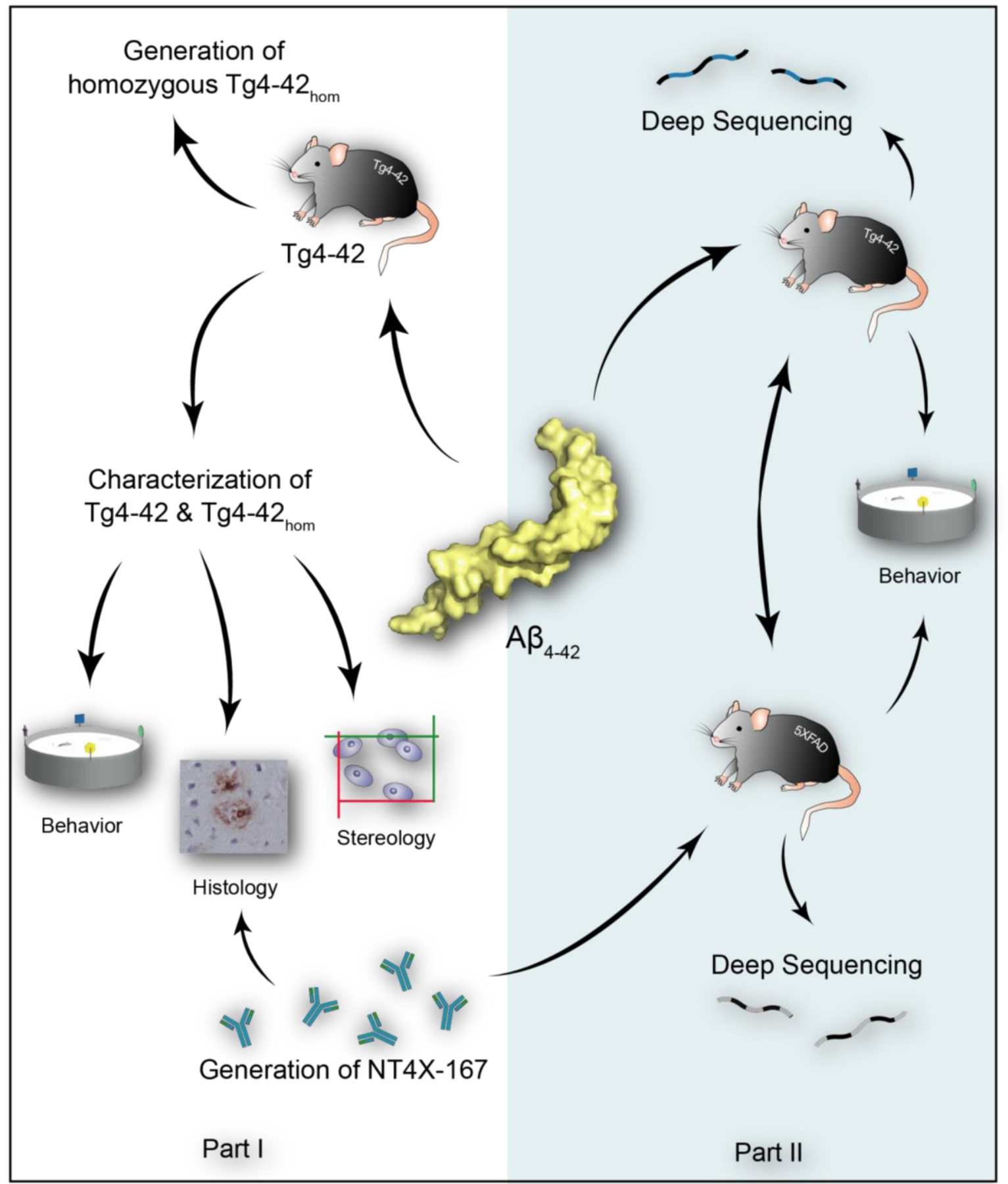

FIGURE 1.9 Schematic overview of the projects presented in this thesis. The thesis is divided in two parts with $A \beta_{4-42}$ as the focus of interest. Parts of project 1 have been publishes in Bouter et al., 2013 and Antonios et al., 2013. Project 2 has recently been published in Bouter et al., 2014. 


\section{MATERIAL AND METHODS}

Animals

2.1.1

General considerations and housing

All animals used in this study were of the species Mus musculus (common house mouse). Wild-type mice were of the inbred strain C57BI/6J (Jackson Laboratories, Bar Harbor, ME, USA). All mice were bred and housed under specific-pathogen-free (SPF) conditions in the central animal facility of the University Medicine Göttingen. Mice were kept in individually ventilated cages (IVC, $32 \times 16 \times 14 \mathrm{~cm}$; Tecniplast, Hohenpeißenberg, Germany) in groups up to five. Water and food were available ad libitum.

All animals were handled according to the guidelines of the 'Society for Laboratory Animals Science' (GV-SOLAS) and the guidelines of the 'Federation of European Laboratory Animal Science Association' (FELASA). Furthermore, all experiments were approved by the 'Lower Saxony State Office for Consumer Protection and Food Safety' (LAVES). All efforts were made to minimize suffering and the number of animals used for this study. If not stated otherwise males and female mice were used for all test.

\subsubsection{Tg4-42 transgenic mice}

Tg4-42 mice were previously generated in our lab and express human $A \beta_{4-42}$ fused to the murine TRH signal peptide under the control of the neuronal Thy-1 promoter (FIGURE 2.1) (Wittnam, 2012; Bouter et al., 2014).

In addition to hemizygous Tg4-42 mice, a homozygous Tg4-42 mouse line was generated and named Tg4-42 hom. Therefore, hemizygous mice were crossed to produce potential homozygous mice. Subsequently, real-time quantitative PCR (qRT-PCR, section 2.3.5.2) was performed on tail-derived DNA (section 2.3.1) to validate homozygosity. Presumptive homozygous mice were backcrossed with wild-type mice ( $\mathrm{C} 57 \mathrm{BI} / 6 \mathrm{~J})$ to test for true homozygosity. Once homozygous males and females were confirmed, they were 
re-mated to each other as the founders for the new homozygous transgenic strain Tg4-42 hom.

Tg4-42 transgene

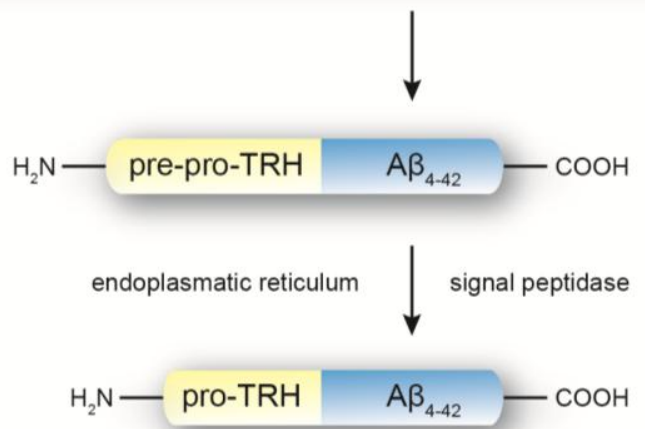

Pro-peptide

$$
\mathrm{H}_{2} \mathrm{~N}-\text { pro-TRH } \quad \mathrm{A}_{4-42}-\mathrm{COOH}
$$

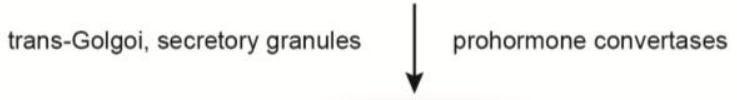

$A \boldsymbol{\beta}_{4-42}$

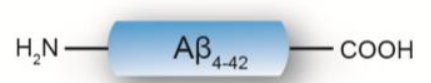

FIGURE 2.1 The Tg4-42 transgene. The murine Thy1 promoter drives the neuronal expression of the pre-pro-TRH-A $\beta_{4-42}$ fusion peptide. $A \beta_{4-42}$ is fused to pre-pro-TRH for liberation within the secretory pathway. An N-terminal signal sequence routes the pre-pro-TRH-A $\beta_{4-42}$ fusion peptide into the endoplasmic reticulum where a signal peptidases releases the pro-TRH-A $\beta_{4-42}$ peptide. In the trans-Golgi and secretory vesicles the rest of the TRH signal peptide is cleave by prohormone convertases. $A \beta_{4-42}$ is released and can be secreted from the cell (Alexandru et al., 2011; Wittnam, 2012). Figure generated after Wittnam, 2012.

\subsection{3}

\section{XFAD transgenic mice}

5XFAD mice overexpress the 695 amino acids isoform of the human amyloid precursor protein (APP695) carrying the Swedish, London and Florida mutations under the control of the murine Thy1-promoter (Vidal et al., 1990; Moechars et al., 1996; Oakley et al., 2006). In addition, human presenilin-1 (PSEN-1) carrying the M146L/L286V mutations is also expressed under the control of the murine Thy1-promoter (Oakley et al., 2006). 5XFAD mice used in the current study were backcrossed for more than eight generations to C57BI/6J wild-type mice (Jackson Laboratories, Bar Harbor, ME, USA) to obtain an incipient congenic line on a C57BI/6J genetic background (Jawhar et al., 2010). Female hemizygous 5XFAD mice and male homozygous 5XFAD mice were used in this study as indicated the results part. 


\subsection{4}

Preparation of mouse brain tissue for biochemistry

Mice were sacrificed via $\mathrm{CO}_{2}$ anesthetization followed by cervical dislocation. Brains were rapidly and carefully dissected on ice after the mouse cadavers were decapitated with a large surgical scissor. Briefly, one incision was made over the dorsal midline of the skull with fine surgical scissors to remove the skin and tissue surrounding the skull. Lateral incisions were made along the temporal bones and a dorsal incision along the median fissure to fracture the skull bones. The skull fragments were removed with tweezers before the whole brain was removed from skull with a small spatula. The cerebellum as well as the olfactory bulb were removed and the brain hemispheres were separated with a surgical scalpel. Brain hemispheres were frozen on dry-ice and stored at $-80^{\circ} \mathrm{C}$ for subsequent use.

\subsubsection{Preparation of mouse brain tissue for immunohistochemistry}

\subsubsection{Drop-fixation}

Mice were sacrificed and the brain was dissected as described in section 2.1.4. The respective brain hemispheres were placed into embedding cassettes (Simport, Beloeil, QC, Canada). Cassettes were stored for at least 96 hours in a histofix solution containing $4 \%$ formalin prepared in phosphate buffered saline (Roth, Karlsruhe, Germany) at $4{ }^{\circ} \mathrm{C}$ until being embedded in paraffin (Roth, Karlsruhe, Germany) (Section 2.4.1) using an EG1140 H Embedding Station (Leica, Wetzlar, Germany).

\subsubsection{Perfusion}

A fast and uniform fixation of mouse tissue was achieved through perfusion via the vascular system through the heart. The perfusion pump was set up and a sterile perfusion needle was attached to one end of the pump's tube. Approximately $20 \mathrm{ml} 0.01$ Dulbecco's phosphate buffered salt solution (PBS; PAN-Biotech, Aidenbach, Germany) were run through the tubing to remove any residues and air bubbles. Mice were anesthetized with a mixture of $10 \%$ ketamine (Medistar, Ascheberg, Germany) and $2 \%$ xylariem (Xylazinhydrochlorid, $23.3 \mathrm{mg} / \mathrm{ml}$; Ecuphar, Oostkamp, Belgium) in molecular-grade water. The anesthetic mixture was given intraperitoneally at a dosage of $100 \mathrm{mg} / \mathrm{kg}$ ketamine and $10 \mathrm{mg} / \mathrm{kg}$ xylazine body weight. After mice were fully anesthetized and showed no pinch-response they were placed on the perfusion board. Mice were pinned by their limbs to the perfusion board. The abdominal wall of the mouse was opened with surgical scissors. The diaphragm and the connective tissue at the bottom of diaphragm 
were cut to allow access to the rib cage. The rib cage was opened to allow access to the beating heart. While holding the heart steady with forceps the needle attached to the tubing of the peristaltic pump was directly inserted into the left ventricle. An incision was made in the right atrium to allow for blood to drain from the circulatory system. Then the mouse was perfused with $50 \mathrm{ml}$ ice-cold $0.01 \mathrm{M}$ PBS. When blood was cleared from the body, perfusion continued with $50 \mathrm{ml}$ ice-cold $4 \%$ paraformaldehyde (PFA; Roth, Karlsruhe, Germany). Spontaneous movement of the tail and discoloring of the liver indicated a successful perfusion.

After perfusion the mouse was removed from the perfusion board and decapitated. Brain tissue was prepared as described as in section 2.1.4. The right hemisphere of the brain was embedded in paraffin (section 2.4.1) while the left hemisphere (including the cerebellum) was cryoprotected. Therefore the brain was first post-fixed in $10 \mathrm{ml}$ PFA overnight at $4{ }^{\circ} \mathrm{C}$. The tissue was then transferred to $10 \mathrm{ml} 30 \%$ sucrose (Roth, Karlsruhe, Germany) solution prepared in $0.01 \mathrm{M}$ PBS and stored at $4{ }^{\circ} \mathrm{C}$ until the brain sunk to the bottom of the container. The brain was removed from the sucrose solution, frozen on dry ice and stored at $-80{ }^{\circ} \mathrm{C}$ for subsequent use.

\section{2}

\section{Behavior analyses of mice}

For phenotyping, mice were kept on a 12 hour / 12 hour inverted light cycle. All behavior experiments were performed during the dark phase between 8 a.m. and 8 p.m..

Weight gain of mice was monitored as part of a general physical assessment. Furthermore, a clasping test was performed to test for functional impairments caused by spinal cord pathology (Jawhar et al., 2010). In order to assess motor and memory deficits in transgenic mice a battery of several tests was performed. Motor coordination of mice was measured by the balance beam and string suspension task. To evaluate behavior and memory, mice were subjected to cross maze, elevated plus maze, Morris water maze and fear conditioning tasks. During a single day of testing mice were subjected to no more than one motor and one memory test. If not stated otherwise males and female mice were used for all tests. 


\subsubsection{Paw-clasping test}

Mice were suspended by their tail for $30 \mathrm{sec}$ to provoke a clasping phenotype. Clasping behavior was scored on a scale from zero to three, where 0 demonstrates no clasping behavior, 1 = forepaws clasping, 2 = one hind paw and forepaws clasping, and $3=$ clasping of all paws (Miller et al., 2008). Healthy mice generally do not show any clasping behavior and rather try to escape the grip by kicking their paws and twisting their whole body.

\subsubsection{String suspension}

Motor coordination and grip strength were assessed using the string suspension task (Hullmann, 2012; Arendash et al., 2001b). The string suspension apparatus consists of a $50 \mathrm{~cm}$ cotton string ( $2 \mathrm{~mm}$ in diameter) tied between two wooden beams $35 \mathrm{~cm}$ above a padded surface. Mice were permitted to grasp the string by their forepaws and then released. Their ability to climb across the string was assessed using a 0 to 5 rating score (TABLE 2.1) (Moran et al., 1995). During a single day of testing each mouse was given three 60 -second trials with a minimum of 10 minutes between the trials. The average score of all three trials was taken as the score for each mouse. Between each trial the apparatus was cleaned with 70 \% ethanol (Merck, Darmstadt, Germany) to diminish odor cues.

TABLE 2.1 String suspension rating score (Moran et al. 1995)

\begin{tabular}{ll}
\hline Score & Description \\
\hline 0 & falls of string \\
1 & hangs onto string by fore- or hindpaws \\
2 & hangs onto string by fore- or hindpaws and attempts to climb onto string \\
3 & hangs onto string by all four paws but no lateral movement \\
4 & hangs onto string using all four paws and tail and moves laterally \\
5 & escapes to the edge of string and touches wooden support beam \\
\hline
\end{tabular}

\subsubsection{Balance Beam}

Fine motor coordination and balance of mice were assessed using the balance beam test (Hau and Schapiro, 2002; Luong et al., 2011). The balance beam consisted of a $1 \mathrm{~cm}$ wide and $50 \mathrm{~cm}$ long dowel attached to two support columns $44 \mathrm{~cm}$ above a padded surface. A $9 \times 15 \mathrm{~cm}$ escape platform was attached to both ends of the beam. The test 
essentially examines the ability of a mouse to remain upright and to walk on the relatively narrow and elevated beam to one of the platforms. During a single day of testing each mouse was given three 60 -second trials with a minimum of 10 minutes between the trials. The average time of all three trials was taken as the score for each mouse. A test trial was given to familiarize the mouse with the beam. For each trial the mouse was placed in the center of the beam facing one of the platforms and then released. The latency to fall from the beam was recorded. If a mouse escaped to one of the platforms or remained on the beam for the entire trial, the maximum time of 60 seconds was given. Between the trials, the beam was cleaned with $70 \%$ ethanol (Merck, Darmstadt, Germany) to reduce odor cues.

\subsection{4}

\section{Cross maze}

Working memory in mice was assessed by analyzing their spontaneous alternation behavior in the cross maze. The spontaneous alternation paradigm is based on the natural tendency of mice to explore new environments: mice tend to alternate between the arms of a maze and typically prefer to explore the least recently visited arm of the maze rather than returning to the one previously visited (Wietrzych et al., 2005).

The cross maze (FIGURE 2.2 A) consisted of four arms $(30 \mathrm{~cm}$ length $\times 8 \mathrm{~cm}$ width $\times 15 \mathrm{~cm}$ height) arranged in $90^{\circ}$ angles extending from a central region (8 cm length $x 8 \mathrm{~cm}$ width $\times 15 \mathrm{~cm}$ height). The whole apparatus was constructed of black plastic. Each mouse was placed randomly at the end of one arm (facing the wall of the maze) to freely explore the maze for a single $10 \mathrm{~min}$ trial, with the experimenter out of the animal's sight. ANY-Maze video tracking software (Stoelting Co, Wood Dale, IL, USA) was used to record the sequence of arm entries and the distance travelled. Thereby, a successful arm entry was counted when all four paws of the mouse crossed the entrance of an arm and an immediate arm re-entry was defined as a single arm entry. Alternations were defined as successive entries into the four arms in overlapping quadruple sets (e.g., 1, 3, 2, 4 or 2, 3, 4, 1 but not 1, 2, 3, 1) (Arendash et al., 2001b). The alternation percentage was calculated as the percentage of actual alternations to the possible number of alternations (= total arm entries - 3) (Wietrzych et al., 2005). The cross maze was cleaned with $70 \%$ ethanol (Merck, Darmstadt, Germany) between mice to reduce odor cues. 
A

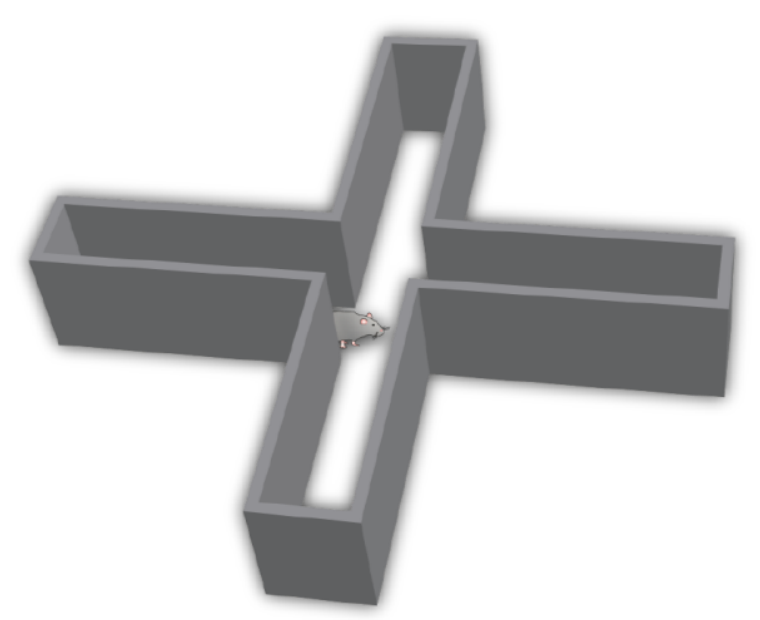

B

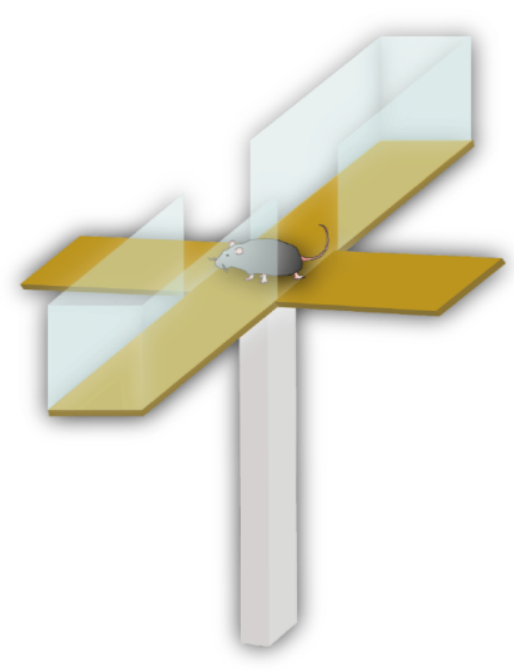

FIGURE 2.2 Cross maze and elevated plus maze. (A) Working memory in mice can be assessed in the cross maze. Typically mice prefer to visit a new arm of the maze rather than returning to the one that was previously visited (B) Anxiety-related behavior can be analyzed in the elevated plus maze. The maze contains open and enclosed arms. Mice will naturally spend most of their time in the enclosed arms due to their reluctance to open spaces.

\subsection{5}

Elevated plus maze

The elevated plus maze test was used to assess anxiety-related behavior in mice (Campos et al., 2013). The test is based on the exploration behavior of mice and their aversion for open spaces (Komada et al., 2008).

The apparatus consisted of four arms (15 cm length $\times 5 \mathrm{~cm}$ width) extending at $90^{\circ}$ angles from a central area (5 cm length $\times 5 \mathrm{~cm}$ width) raised $75 \mathrm{~cm}$ above a padded surface (FIGURE 2.2 B). The maze was composed of two oppositely positioned enclosed arms that were surrounded by a $15 \mathrm{~cm}$ high translucent plastic wall on three sides and two open arms. Mice were placed in the central area of the maze facing one of the open arms and allowed to freely explore the apparatus for 5 minutes. ANY-Maze video tracking software (Stoelting Co, Wood Dale, IL, USA) was used to record the distance travelled and the percentage spent in the open arms. Anxiety-like behavior can be measured by the time spent in the open arms as lower anxiety levels correspond to longer time spent in open arms (Karl et al., 2003). Between mice the maze was cleaned with $70 \%$ ethanol (Merck, Darmstadt, Germany) to diminish odor cues. 


\subsection{6}

Morris water maze

Spatial reference memory of mice was evaluated using the Morris water maze (Morris, 1984). In this task, mice learn to use spatial cues to locate a hidden, circular platform (10 $\mathrm{cm}$ ) in a circular pool (110 cm diameter) filled with tap water. The water was made opaque by adding non-toxic white paint and maintained at $20^{\circ} \mathrm{C} \pm 2^{\circ} \mathrm{C}$ for the whole test duration. The pool was divided into four virtual quadrants that were defined based on their spatial relationship to the platform: left, right, opposite and target quadrant, which contains the goal platform. ANY-Maze video tracking software (Stoelting Co, Wood Dale, IL, USA) connected with a camera (Computar, Commack, NY, USA) was used to record escape latency, path length, swimming speed and quadrant preference. In order to minimize the number of animals that were tested hemizygous Tg4-42 mice were examined together with Katharina Dietrich.

A

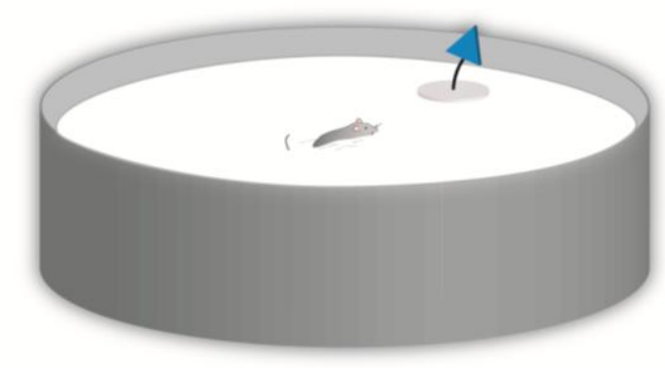

Day 1 - 3
B

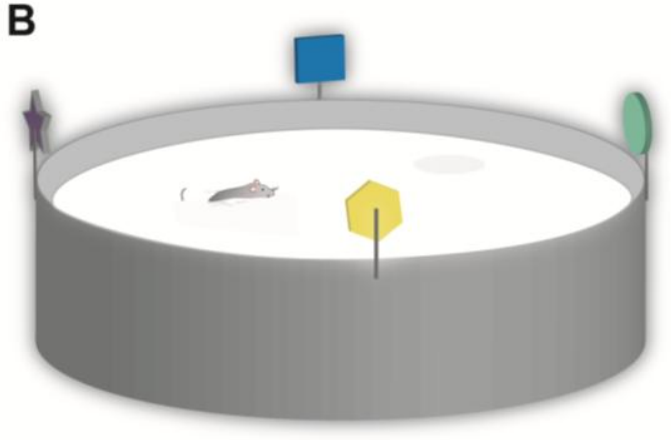

Day 4 - 8

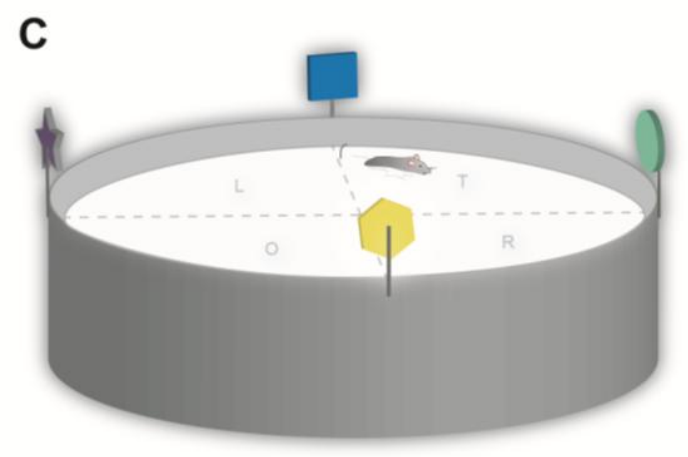

Day 9

FIGURE 2.3 Morris Water Maze. (A) Mice perform 3 days of cued training. During these trials the escape platform is marked with a triangular flag. (B) For the five days of acquisition training the flag is removed from the platform. Proximal visual cues are attached to the outside of the pool. (C) During a single probe trial the platform is removed from the pool. Mice are allowed to swim freely for one minute while their swimming path is recorded. Abbreviations for quadrants: $T=$ target, $\mathrm{L}=$ left, $\mathrm{R}=$ right, $\mathrm{O}=$ opposite. 
Testing began with three days of cued training (FIGURE 2.3 A). For these trials the platform was marked with a triangular flag. Mice were introduced into the water at the edge of the pool facing the wall. They were then given one minute to find the submerged platform. Mice that failed to find the platform in 60 seconds were gently guided to it. All mice were allowed to sit on the platform for 10 seconds before being removed from the pool. To prevent hypothermia, all mice were kept in front of a heat lamp for 3 minutes before being returned to their home cage. Each mouse received four training trials per day with an average inter-trial interval of 15 minutes. Both the location of the platform and the position at which mice were introduced into the pool changed between trials. All mice had to show a significantly increased learning curve or find the platform in under 15 seconds in order to continue testing.

Twenty-four hours after the last day of cued training, mice performed five days of acquisition training. For this part of testing, the flag was removed from the platform (FIGURE 2.3 B). In addition to the distal cues existing in the room proximal visual cues were attached to the outside of the pool. The platform location remained stationary for each mouse throughout training. At the start of every trial, mice were introduced into the pool from one of four predefined entry points. The order in which these entry points were used varied between training days (Vorhees and Williams, 2006). To avoid quadrant bias, the experimental cohorts were randomly split and trained to find one of two different platform locations. Trials were conducted as during the cued training phase.

Twenty-four hours after the last acquisition trial, a probe test was performed to assess spatial reference memory. The platform was removed from the pool (FIGURE 2.3 C) and mice were introduced into the water from a novel entry point. Mice were then allowed to swim freely for one minute while their swimming path was recorded. Mice that successfully acquired a spatial search strategy show a clear preference for the goal quadrant.

\subsection{7}

\section{Contextual and Tone Fear Conditioning}

Mice were subjected to contextual fear conditioning (CFC) and tone fear conditioning (TFC). A three-day delay fear conditioning protocol was used to assess conditional learning and memory. Thereby, the conditioned stimulus (CS) is presented and overlapped by the presentation of the unconditioned stimulus (US) (Ohno, 2009).

The experiments were performed using a standard conditioning chamber $(17 \mathrm{~cm} \mathrm{x}$ $17 \mathrm{~cm} \times 26 \mathrm{~cm}$ ) with a stainless steel grid floor connected to a shock generator (Ugo Basile Sound and Shocker Generator, Comerio, Italy). The walls were covered with black and white checkered paper (CS). The chamber was housed in a soundproof isolation 
cubicle. A digital camera (Unibrain, San Ramon, CA, USA) and an additional light source were attached to the ceiling of the cubicle. ANY-Maze video tracking software (Stoelting Co.,Wood Dale, IL, USA) was used to record freezing behavior of animals.

On day one, mice were placed in the conditioning chamber and allowed to explore the box for 150 seconds. After the habituation period a tone $(2000 \mathrm{~Hz}, 80 \mathrm{~dB}$; CS) was presented for 30 seconds that simultaneously ended with a two second foot-shock $(0.7$ mA, US; FIGURE 2.4 A). Mice were allowed to recover after the foot-shock for an additional 30 seconds before being returned to their home cage. Baseline freezing was recorded before the presentation of the tone/shock.

Twenty-four hours after the training mice were placed back in the familiar fear conditioning chamber, but in the absence of tone and foot-shocks (FIGURE 2.4 B). Freezing behavior was measured for 210 seconds to test contextual memory retrieval.

For the tone fear retrieval trial on day three, mice were placed for three minutes in an altered conditioning chamber with white walls, a covered floor and an acetic acid scent. After 150 seconds baseline recording, a tone similar to the one used during the fear conditioning trial was presented for 30 seconds (FIGURE $2.4 \mathrm{C}$ ). The freezing behavior before and during the conditioned stimulus tone was measured. Freezing is characterized by the absence of movement apart from respiration and shivering in combination with crouching (Wehner and Radcliffe, 2004). Between mice the maze was cleaned with $70 \%$ ethanol (Merck, Darmstadt, Germany) to diminish odor cues.

A

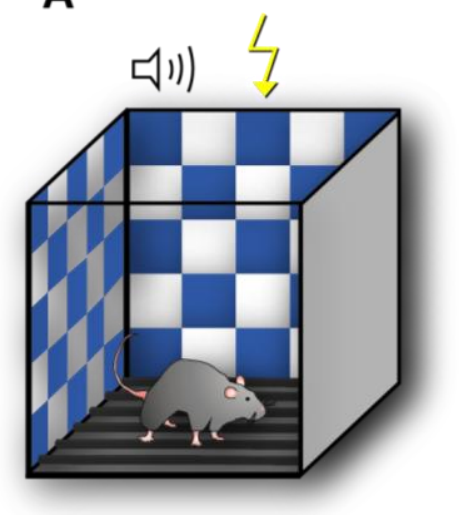

B

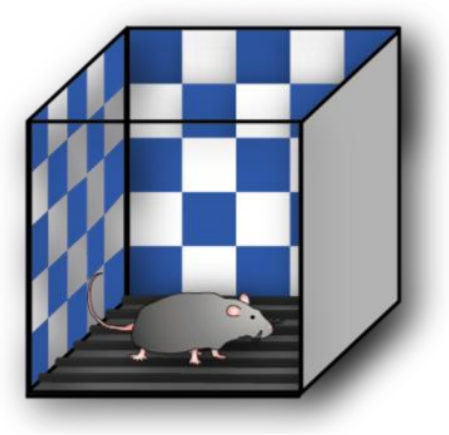

C

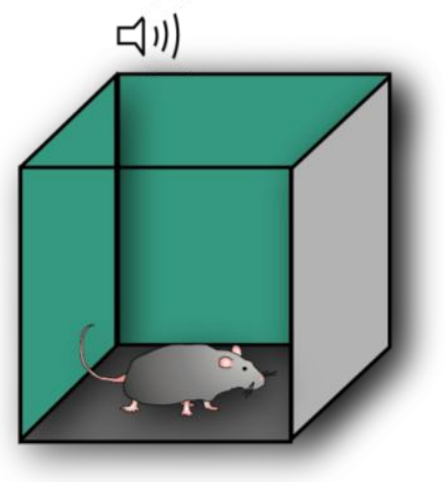

FIGURE 2.4 Fear conditioning. (A) During the training trial a tone is presented for 30 seconds that simultaneously ends with a two second foot-shock. (B) 24 hours after the training trial mice are placed back in the familiar fear conditioning chamber to test for contextual learning. No tone and foot-shock is presented. (C) 48 after the training trial mice are placed in an altered conditioning chamber. $A$ tone is presented to test for tone learning $(A-C)$ Freezing is recorded at all times. 


\subsection{1}

\section{DNA isolation from mouse tails}

Prior further use in any experiment all mice were genotyped. For genotyping DNA was isolated from mouse tail biopsies. $500 \mu \mathrm{l}$ lysis buffer $[100 \mathrm{mM} \mathrm{Tris} / \mathrm{HCl}(\mathrm{pH} 8.5$; Roth, Karlsruhe, Germany), 5 mM EDTA (AppliChem, Darmstadt, Germany), $0.2 \%$ sodium dodecyl sulfate (SDS; Biomol, Hamburg, Germany) 200 mM NaCl (Roth, Karlsruhe, Germany)] and $5 \mu \mathrm{L}$ Proteinase $\mathrm{K}(20 \mathrm{mg} / \mathrm{ml}$ stock; Peqlab, Erlangen, Germany) were added to every tail biopsy. Samples were incubated for 20 hours at $55^{\circ} \mathrm{C}$ and $400 \mathrm{rpm}$ in a Thermomixer Compact (Eppendorf, Hamburg, Germany). After incubation samples were centrifuged for 20 minutes at $4^{\circ} \mathrm{C}$ and $17,000 \mathrm{rpm}$ (Heraeus Biofuge Stratos, Thermo Fisher Scientific, Waltham, MA, USA). The supernatant was transferred into a new $1.5 \mathrm{ml}$ Eppendorf tube containing $500 \mu \mathrm{l}$ cold isopropanol (Roth, Karlsruhe, Germany). Samples were vortexed (Vortex Genie 2, Scientific Industries, Bohemia, NY, USA) until DNA precipitate was visible and centrifuged for 10 minutes at $13,000 \mathrm{rpm}$ at room temperature (RT) (Biofuge Pico, Heraeus). After centrifugation, the supernatant was discarded and the remaining DNA pellet was washed with $500 \mu \mathrm{l}$ cold $70 \%$ absolute ethanol (Merck, Darmstadt, Germany). Following another centrifugation step at 13,000 rpm for 10 minutes at RT, the supernatant was discarded and the pellet dried at $37^{\circ} \mathrm{C}$ in a Thermomixer Compact for 45 minutes. The DNA pellet was resuspended in $30 \mu \mathrm{l}$ molecular grade water (Braun, Melsungen, Germany) and dissolved overnight at $55{ }^{\circ} \mathrm{C}$ in a Thermomixer Compact before being stored at $4{ }^{\circ} \mathrm{C}$. DNA concentration was determined via photometry (Eppendorf, Hamburg, Germany) at OD260 (section 2.3.3) and samples were diluted to a concentration of $20 \mathrm{ng} / \mu \mathrm{l}$ with molecular grade water.

\subsubsection{RNA isolation from mouse brain}

Isolation of RNA from mouse brains were performed under a fume hood. Brains were homogenized with 10 strokes of a R50D homogenizer (CAT Scientific, Paso Robles, CA, USA) set at $800 \mathrm{rpm}$ in $1.5 \mathrm{ml}$ ice-cold TriFast ${ }^{\circledR}$ (Peqlab, Erlangen, Germany) in a $2.0 \mathrm{ml}$ Eppendorf tube. $300 \mu \mathrm{l}$ Chloroform (Merck, Darmstadt, Germany) was added to each sample and inverted by hand. After 10 minutes incubation the samples were centrifuged at $12,000 \mathrm{rpm}$ for 15 minutes at $4{ }^{\circ} \mathrm{C}$ to separate the RNA. The upper RNA-containing aqueous phase was transferred into a new Eppendorf tube, vigorously mixed with $500 \mu \mathrm{l}$ Isopropanol (Roth, Karlsruhe, Germany) and incubated for 20 minutes on ice. After centrifugation at $12,000 \mathrm{rpm}$ for 10 minutes at $4{ }^{\circ} \mathrm{C}$ the supernatant was discarded. RNA pellets were washed twice with $500 \mu \mathrm{l} 75 \%$ absolute ethanol. After the pellet was air-dried 
the RNA was dissolved in $30 \mu \mathrm{l}$ of with molecular grade water and stored at $4{ }^{\circ} \mathrm{C}$ over night. The next day, RNA was subjected to digestion by DNase. Therefore, $3 \mu l 10 \mathrm{X}$ DNAse Buffer (Thermo Fisher Scientific, Waltham, MA, USA), $2 \mu \mathrm{l}$ DNAse I (1 U/ $\mu$; Thermo Fisher Scientific, Waltham, MA, USA) and $1 \mu \mathrm{L}$ RNasin (40 U/ $\mu \mathrm{l}$; Promega, Fitchburg, WI, USA) were added to $24 \mu$ of each RNA sample. After 90 minutes incubation at $37^{\circ} \mathrm{C} 70 \mu \mathrm{l}$ molecular grade water and $400 \mu \mathrm{l}$ ice-cold TriFast were added to the mixture. The solution was incubated at room temperature for 5 minutes before mixed with $60 \mu \mathrm{l}$ Chloroform. The solution was again incubated at room temperature for 3 minutes and centrifuged at $12,000 \mathrm{rpm}$ for 15 minutes at $4{ }^{\circ} \mathrm{C}$. The clear supernatant RNA phase was transferred into a new $2.0 \mathrm{ml}$ Eppendorf tube. $200 \mu \mathrm{l}$ Isopropanol were added to the solution, mixed vigorously and stored on ice for 15 minutes before being centrifuged at $12,000 \mathrm{rpm}$ for 10 minutes at $4{ }^{\circ} \mathrm{C}$. After centrifugation the supernatant was discarded and the pellet was washed twice with $500 \mu \mathrm{l} 75 \%$ absolute ethanol. After the pellet was air-dried the RNA was resuspended in $30 \mu \mathrm{l}$ of molecular grade water and dissolved at 4 ${ }^{\circ} \mathrm{C}$ over night. RNA purity and yields were determined (section 2.3.3) and RNA was stored at $-80^{\circ} \mathrm{C}$ until further use.

\subsubsection{Nucleic acid quantitation}

DNA and RNA purity and yields were determined by a Biophotometer (Eppendorf, Hamburg, Germany). $80 \mu$ molecular grade water was used as a blank for the photometry settings. Afterwards, $2 \mu$ of each sample was diluted with $78 \mu$ molecular grade water in a Uvette $\AA$ 220-1600 nm cuvette (Eppendorf, Hamburg, Germany). The A260/A230 absorbance ratio and the A260/A280 absorbance ratio were measured in the photometer for each DNA and RNA sample, respectively. For DNA samples A260/A230 and A260/A280 ratios greater than 1.8 were considered as accurate. RNA samples that displayed A260/A230 ratios greater than 1.8 and A260/A280 ratios higher than 2.0 were considered as acceptable pure (Barbas et al., 2007).

\subsection{4}

Detection of DNA

\subsubsection{Polymerase chain reaction (PCR)}

Genomic DNA extracted from tail biopsies (section 2.3.1) was used for genotyping. PCR reactions were performed in a LabCycler (SensoQuest, Goettingen, Germany) using 0.2 $\mu$ I PCR tubes (Greiner Bio-One, Kremsmuenster, Austria). DNA was added to a master mix containing 10X reaction buffer (Axon, Kaiserslautern, Germany), 25 mM MgCl2 (Axon), Taq polymerase (5 $\mathrm{U} / \mu \mathrm{l}$; Axon), dNTPs (Invitrogen), water and the respective 
primers. Reaction mixes and cycling conditions are given in TABLE 2.2, TABLE 2.3 and TABLE 2.4, respectively. Primer details are listed in TABLE 2.7. PCR products were loaded on agarose gels as described in section 2.3.4.2.

TABLE 2.2 Reaction mix for Tg4-42 PCR

\begin{tabular}{lc}
\hline Reagent & Volume \\
\hline DNA (20 ng/ $\mu \mathrm{l})$ & $2.0 \mu \mathrm{l}$ \\
$\mathrm{A} \beta 3$-42 for2 primer & $1.0 \mu \mathrm{l}$ \\
$\mathrm{A} \beta 3$-42 rev2 primer & $1.0 \mu \mathrm{l}$ \\
dNTPs $(2 \mathrm{mM})$ & $2 \mu \mathrm{l}$ \\
$\mathrm{MgCl}(25 \mathrm{mM})$ & $1.6 \mu \mathrm{l}$ \\
$10 \mathrm{X}$ reaction buffer & $2 \mu \mathrm{l}$ \\
ddH ${ }_{2} \mathrm{O}$ & $10.2 \mu \mathrm{l}$ \\
Taq polymerase $(5 \mathrm{U} / \mu \mathrm{l})$ & $0.2 \mu \mathrm{l}$ \\
Total volume per sample & $20 \mu \mathrm{l}$ \\
\hline
\end{tabular}

TABLE 2.3 Reaction mix for 5XFAD PCR

\begin{tabular}{lc}
\hline Reagent & Volume \\
\hline DNA $(20 \mathrm{ng} / \mu \mathrm{l})$ & $2.0 \mu \mathrm{l}$ \\
hAPP for primer & $0.5 \mu \mathrm{l}$ \\
hAPP rev primer & $0.5 \mu \mathrm{l}$ \\
dNTPs $(2 \mathrm{mM})$ & $2 \mu \mathrm{l}$ \\
$\mathrm{MgCl} 2(25 \mathrm{mM})$ & $3.2 \mu \mathrm{l}$ \\
$10 X$ reaction buffer & $2 \mu \mathrm{l}$ \\
ddH ${ }_{2} \mathrm{O}$ & $9.6 \mu \mathrm{l}$ \\
Taq polymerase $(5 \mathrm{U} / \mu \mathrm{l})$ & $0.2 \mu \mathrm{l}$ \\
Total volume per sample & $20 \mu \mathrm{l}$ \\
\hline
\end{tabular}


TABLE 2.4 PCR cycling program for genotyping Tg4-42 and 5XFAD mice

\begin{tabular}{lcc}
\hline Step & Temperature & Duration \\
\hline 1 & $94^{\circ} \mathrm{C}$ & $3 \mathrm{~min}$ \\
2 & $94^{\circ} \mathrm{C}$ & $45 \mathrm{sec}$ \\
3 & $58^{\circ} \mathrm{C}$ & $1 \mathrm{~min}$ \\
4 & $72^{\circ} \mathrm{C}$ & $1 \mathrm{~min}$ \\
5 & repeat steps $2-4$ a total of 35 times \\
6 & $72{ }^{\circ} \mathrm{C}$ & 5 min \\
7 & $4{ }^{\circ} \mathrm{C}$ & $\infty$ \\
\hline
\end{tabular}

\subsubsection{DNA gel electrophoresis}

Agarose gel electrophoresis was used to analyze the PCR products (Section 2.3.4.1). For a $2 \%$ agarose gel $100 \mathrm{ml}$ of 1 XTBE buffer was mixed with $2.0 \mathrm{~g}$ agarose (Lonza, Basel, Switzerland) and boiled in a microwave at $560 \mathrm{~W}$ until the agarose was completely dissolved. Three $\mu$ l ethidium bromide (10 mg/ml; Roth, Karlsruhe, Germany) was added to the mixture. The solution was poured into a casting tray with a 20-pocket sample comb and any air bubbles were removed. After the gel was set the comb was carefully removed and the gel was positioned into an electrophoresis chamber (Bio-Rad, Hercules, CA, USA) filled with 1 XTBE buffer. Ten $\mu$ PCR product was mixed with $1 \mu \mathrm{l} 10 \mathrm{X}$ agarose gel sample buffer (Life Technologies, Carlsbad, CA, USA) and loaded into a well. For sizing 5 $\mu \mathrm{l}$ of a $100 \mathrm{bp}$ DNA ladder (Bioron, Ludwigshafen, Germany) was added into the first well. The gel electrophoresis chamber was connected to a Power Pack P25 power supply (Biometra, Goettingen, Germany) and the gel was run for approximately $45 \mathrm{~min}$ at $120 \mathrm{~V}$. Results were visualized under UV light $(366 \mathrm{~nm}$ ) using the Gel Doc 2000 (Biorad, Hercules, CA, USA) and analyzed with the Quantity One software program (Version 4.30; Biorad).

10XTBE buffer: $108 \mathrm{~g}$ Tris (Roth, Karlsruhe, Germany) and $55 \mathrm{~g}$ boric acid (Sigma, St.

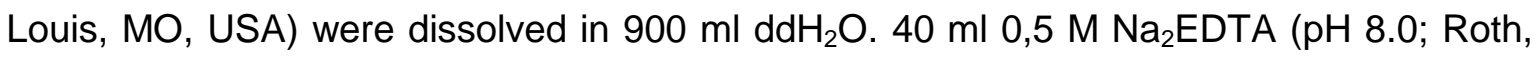
Karlsruhe, Germany) was added to the solution and the volume was adjusted to 1 I with $\mathrm{dd}_{2} \mathrm{O}$. Before use the solution was diluted $1: 10$ in $\mathrm{ddH}_{2} \mathrm{O}$ to obtain 1XTBE buffer. 


\subsection{5}

\section{Detection of RNA}

\subsubsection{Reverse transcription}

Total RNA isolated from mouse brain tissue (section 2.3.2) was subjected to reverse transcription to synthesize cDNA using the First Strand cDNA Synthesis Kit (Fermentas, St. Leon-Rot, Germany) according to the manufacturer's instructions. Therefore, $1 \mu \mathrm{l}$ of random hexamer primer, $4 \mu \mathrm{l} 5 \mathrm{X}$ reaction buffer, $1 \mu \mathrm{l}$ RiboLock® RNase inhibitor (20 U/ $\mu \mathrm{l})$, $2 \mu \mathrm{l}$ of $10 \mathrm{mM}$ dNTP mix and $1 \mu \mathrm{l}$ RevertAid® reverse transcriptase $(20 \mathrm{U} / \mu \mathrm{l})$ were added to $11 \mu$ DNase digested RNA template $(1 \mu \mathrm{g})$. The reaction mixture was incubated 5 minutes at $25^{\circ} \mathrm{C}, 1$ hour at $37^{\circ} \mathrm{C}$ and 5 minutes at $70{ }^{\circ} \mathrm{C}$ in a LabCycle (SensoQuest, Goettingen, German). Generated cDNA was diluted 1:10 in molecular grade water and used as the sample template for qRT-PCR (section 2.3.5.2). The obtained cDNA was stored at $-20^{\circ} \mathrm{C}$ until further use.

\subsubsection{Real-time quantitative PCR (qRT-PCR)}

Analysis of transgene expression in the Tg4-42 line for genotyping and gene expression levels in mice were analyzed through qRT-PCR. The diluted first-strand cDNA or DNA, respectively (section 2.3.5.1 and 2.3.1) were used for qRT-PCR using the SYBR-green based DyNAmo Flash SYBR Green qPCR Kit (Thermo Fisher Scientific, Waltham, MA, USA) containing ROX as an internal reference dye. Reaction mixes and cycling conditions are given in TABLE 2.5 and TABLE 2.6, respectively. cDNA dilutions were mixed with the qPCR reaction mix in $200 \mu \mathrm{l}$ PCR tubes (Biozym, Oldendorf, Germany) and briefly centrifuged in a Spectrafuge Mini (Labnet Inc., Edison, NJ, USA). Samples were normalized to the housekeeping gene $\beta$-Actin. Primers were purchased from Eurofins (Ebersberg, Germany) as intron-spanning validated primer pairs (TABLE 2.7). $\mathrm{C}_{\mathrm{T}}$ values were measurements and all samples were run in duplicates. qRT-PCRs were performed in the MX3000P Real-Time Cycler (Stratagene, Santa Clara, CA, USA) and data collected using the MxPro Mx3000P software (Stratagene, Santa Clara, CA, USA). 
TABLE 2.5 Reaction mix for qRT-PCR

\begin{tabular}{lc}
\hline Reagent & Volume \\
\hline 1:10 cDNA solution $(20 \mathrm{ng} / \mu \mathrm{l})$ & $1.0 \mu \mathrm{l}$ \\
Master Mix & $12.5 \mu \mathrm{l}$ \\
forward primer & $1.5 \mu \mathrm{l}$ \\
reverse primer & $1.5 \mu \mathrm{l}$ \\
$\mathrm{ROX}$ & $0.3 \mu \mathrm{l}$ \\
$\mathrm{ddH}_{2} \mathrm{O}$ & $8.2 \mu \mathrm{l}$ \\
Total volume per sample & $25 \mu \mathrm{l}$ \\
\hline
\end{tabular}

TABLE 2.6 qRT-PCR cycling program

\begin{tabular}{|c|c|c|}
\hline Step & Temperature & Duration \\
\hline 1 & $95^{\circ} \mathrm{C}$ & $10 \min$ \\
\hline 2 & $95^{\circ} \mathrm{C}$ & $15 \mathrm{sec}$ \\
\hline 3 & $58^{\circ} \mathrm{C}$ & $30 \mathrm{sec}$ \\
\hline 4 & $72^{\circ} \mathrm{C}$ & $30 \mathrm{sec}$ \\
\hline 5 & \multicolumn{2}{|c|}{ repeat steps 2 - 4 for 39 cycles } \\
\hline 6 & $95^{\circ} \mathrm{C}$ & $1 \mathrm{~min}$ \\
\hline 7 & $55^{\circ} \mathrm{C}$ & $30 \mathrm{sec}$ \\
\hline 8 & $95^{\circ} \mathrm{C}$ & $30 \mathrm{sec}$ \\
\hline
\end{tabular}

\subsection{6}

Primers

All primers were used at a concentration of $10 \mathrm{pmol} / \mu \mathrm{l}(1: 10$ dilution of the $100 \mathrm{pmol} / \mu \mathrm{l}$ primer stock prepared in $\mathrm{dd}_{2} \mathrm{O}$ ). Primers were purchased from Eurofins (Ebersberg, Germany) as intron-spanning validated primer pairs. 
TABLE 2.7 List of primers used for mouse genotyping and qRT-PCR (in order of appearance)

\begin{tabular}{|c|c|c|}
\hline Name & Sequence $\left(5^{\prime} \rightarrow 3^{\prime}\right)$ & Used for \\
\hline$A \beta 3-42$ for & GTGACTCCTGACCTTCCAG & $\begin{array}{l}\text { Genotyping (Amplicon size } 248 \text { bp), } \\
\text { qRT-PCR }\end{array}$ \\
\hline $\mathrm{A} \beta 3-42 \mathrm{rev}$ & GTTACGCTATGACAACACC & $\begin{array}{l}\text { Genotyping (Amplicon size } 248 \text { bp), } \\
\text { qRT-PCR }\end{array}$ \\
\hline hAPP for & GTAGCAGAGGAAGAAGTG & Genotyping (Amplicon size 250 bp) \\
\hline hAPP rev & CATGACCTGGGACATTCTC & Genotyping (Amplicon size 250 bp) \\
\hline Aldoa for & AGCTCСTTCTTCTGCTCCG & qRT-PCR \\
\hline Aldoa rev & TTAGTCCTTTCGCCTACCCA & qRT-PCR \\
\hline Rps2 for & TTGGAGATGGCATCAAAGGT & qRT-PCR \\
\hline Rps2 rev & ATTGTCTCTGCTCCTGTGCC & qRT-PCR \\
\hline Ide for & CAGGCATCGTTCATCACATT & qRT-PCR \\
\hline Ide rev & ACAGGTTTGCGCAGTTTTTC & qRT-PCR \\
\hline Rpl7a for & TCTTGCTTTGTCTCTGGCCT & qRT-PCR \\
\hline Rpl7a rev & ATCCTCTACAAGCGGCTCAA & qRT-PCR \\
\hline Ttr for & GGTGCTGTAGGAGTATGGGC & qRT-PCR \\
\hline Ttr rev & GGAAGACACTTGGCATTTCC & qRT-PCR \\
\hline Sgk1 for & CCGTGTTCCGGCTATAAAAC & qRT-PCR \\
\hline Sgk1 rev & GCCTGAGTATCTGGCTCCTG & qRT-PCR \\
\hline Rpl21 for & TTCCTTTTTGAACAGTGCCC & qRT-PCR \\
\hline Rpl21 rev & GCACCCGGTACATGTTCTCT & qRT-PCR \\
\hline Pafah1b2 for & GAATAGCTGCTGGGTTCGAG & qRT-PCR \\
\hline Pafah1b2 rev & GATCAAGCGAGCGACTGAA & qRT-PCR \\
\hline Psap for & ACAGTCCACCGCCGTCTT & qRT-PCR \\
\hline Psap rev & CTCTTCGCCAGCCTTCTG & qRT-PCR \\
\hline Ubqln2 for & TTGAGCTGTTCCAGTTGCTG & qRT-PCR \\
\hline Ubqln2 rev & ACCCAACCAGCAGTTCATTC & qRT-PCR \\
\hline Spnb3 for & CTGTACTGGCCCTGGATCTC & qRT-PCR \\
\hline Spnb3 rev & CTTTGAAAAGGAAACGGAGG & qRT-PCR \\
\hline GFAP for & CCTTCTGACACGGATTTGGT & qRT-PCR \\
\hline GFAP rev & ACATCGAGATCGCCACCTAC & qRT-PCR \\
\hline
\end{tabular}




\begin{tabular}{lll} 
Atp1a3 for & CATCCAGCAGAATCATGTCG & qRT-PCR \\
Atp1a3 rev & GATGGTGTGAATGACTCCCC & qRT-PCR \\
Calm3 for & TCCTTGTCGAAGAGGGAGAA & qRT-PCR \\
Calm3 rev & CAGAGTAACCTCGACCCCC & qRT-PCR \\
Fbxo2 for & CACATCTTCGTTCTCCGACA & qRT-PCR \\
Fbxo2 rev & TGGGAGGAGCTACTGGACAC & qRT-PCR \\
Aldoa for & AGCTCCTTCTTCTGCTCCG & qRT-PCR \\
Aldoa rev & TTAGTCCTTTCGCCTACCCA & qRT-PCR \\
Gmp6a for & GGAATACCTCCCAGGCATTTA & qRT-PCR \\
Gmp6a rev & ACTAATACCGTGCGCCAGAT & qRT-PCR \\
Ncor2 for & TACACTGGGACACGGTCTTG & qRT-PCR \\
Ncor2 rev & AGTTCTCGCTGGACTGAGGA & qRT-PCR \\
Firt1 for & CAGAGTGTGCCACCACCAT & qRT-PCR \\
Firt1 rev & GAAGCAGTGACCAGTGGGAC & qRT-PCR \\
Dcx for & TCATCTTGAGCATAGCGGAA & qRT-PCR \\
Dcx rev & GGAAACCGGAGTTGTCAAAA & qRT-PCR \\
Syn3 for & GTCCTGGTAGTCGTGCTGGT & qRT-PCR \\
Syn3 rev & ACATTTTTCCCCAACCACAA & qRT-PCR \\
\hline
\end{tabular}

\section{Detection of Peptides}

\subsubsection{1}

\section{Monomerization of Synthetic Peptides}

The amyloid $\beta$ variants $A \beta_{4-38}, A \beta_{4-40}, A \beta_{4-42}, A \beta_{p E 3-42}$ and $A \beta_{1-42}$ were monomerized (Peptide Specialty Laboratory PSL, Heidelberg, Germany). First, peptides were dissolved in $10 \mathrm{mM} \mathrm{NaOH}$ at a concentration of $1 \mathrm{mg} / \mathrm{ml}$ and aliquoted in $50 \mu \mathrm{l}$ volumes. For monomerization, peptides were sonicated for 5 minutes (Sonorex RK $100 \mathrm{H}$; Bandelin electronic, Berlin, Germany), flash-frozen in liquid nitrogen and stored at $-80{ }^{\circ} \mathrm{C}$ until further use.

\subsubsection{Western Blot Analyses}

For Western blot analysis under reducing conditions, $1 \mu \mathrm{g}$ monomerized peptides (section 2.3.7.1) were mixed 1:1 with Vario Gel SDS loading buffer (Anamed, Rodau, Germany) in $0.5 \mathrm{ml}$ reactions tubes and incubated for 10 minutes at $95{ }^{\circ} \mathrm{C}$ in an Uno-Thermoblock 
(Biometra, Goettingen, Germany). Samples were loaded on 4 - $12 \%$ sodium dodecylsulfate-polyacrylamide VarioGels (Anamed) in a X-Cell II TM SureLock chamber (Invitrogen, Carlsbad, CA, USA) filled with $500 \mathrm{ml}$ 1X Tricin SDS running buffer (Anamed). Additionally, $5 \mu$ low and broad range marker (Thermo Fisher Scientific, Waltham, MA, USA) were loaded on each gel. Electrophoresis was performed at $60 \mathrm{~V}$ for 45 minutes followed by $120 \mathrm{~V}$ for 1 hour. After electrophoresis, gels were immersed in transfer buffer and proteins were transferred to $0.45 \mu \mathrm{m}$ Hybond-ECL nitrocellulose membranes (GE Healthcare, Chalfont St. Giles, GB) by semi-dry blotting at $25 \mathrm{~V}$ for $45 \mathrm{~min}$ in a Trans-Blot SD Semi-Dry Transfer cell (Bio-Rad, Hercules, CA, USA). Membranes were washed twice for 10 minutes in 1XTBS-T (0.01 M TBS containing $0.05 \%$ Tween) and heated for 5 minutes in 0.01 PBS ( $800 \mathrm{~W}$ until boiling followed by $80 \mathrm{~W}$ ) to allow better $A \beta$ detection. Membranes were blocked in $10 \%$ non-fat dry milk (Roth, Karlsruhe, Germany) in TBS-T over night at $4{ }^{\circ} \mathrm{C}$ on a nutating shaker (GFL, Burgwedel, Germany). On the second day, membranes were incubated with the primary antibody of interest (TABLE 2.8) in TBS-T for 5 hours at RT. Membranes were washed twice with the TBS-T, incubated with the appropriate peroxidase-conjugated secondary antibody (TABLE 2.9) for 1.5 hours at RT and washed again twice with the TBS-T. Blots were developed using chemiluminescence solution Luminata Crescendo Western HRP substrate (Merck, Darmstadt, Germany) and standard emulsion film (Kodak, Rochester, NY, USA) in a Curix60 developing machine (Agfa, Mortsel, Belgium).

\section{4}

\section{Immunohistochemistry}

\subsection{1}

\section{Paraffin embedding and sectioning of mouse brain}

After fixation of brain tissue (section 2.1.5.1) embedding cassettes containing the tissue were placed in the TP 1020 Automatic Tissue Processor (Leica, Wetzlar, Germany) for dehydration and paraffin immersion. The following dehydration protocol was used: 5 minutes in $4 \%$ histofix (Roth, Karlsruhe, Germany); 30 minutes in tap water; 1 hour in 50 $\%, 60 \%, 70 \%, 80 \%$ and $90 \%$ EtOH solutions (CVH, Hannover, Germany); twice 1 hour in $100 \% \mathrm{EtOH} ; 1$ hour in xylol (Roth) and twice 1 hour in melted paraffin (Roth). After dehydration samples were kept in melted paraffin for 1 hour before embedded in paraffin blocks using an EG1140 H Embedding Station (Leica).

Paraffin embedded brains were cut in $4 \mu \mathrm{m}$ sagittal sections using a $\mathrm{HM} 335 \mathrm{E}$ microtome (Thermo Fisher Scientific, Waltham, MA, USA). Sections were mounted onto Superfrost ${ }^{\circledR}$ slides (Thermo Fisher Scientific) in a $\mathrm{dd}_{2} \mathrm{O}$ water bath at RT. Then the sections were fixed onto the slides by dipping them in a $52{ }^{\circ} \mathrm{C}$ water bath (Medax, 
Olching, Germany). Before being used for immunohistochemistry (sections 2.4 .2 and 2.4.3) slides were dried for 10 minutes on a $52{ }^{\circ} \mathrm{C}$ hot plate (Medax) and overnight at 37 ${ }^{\circ} \mathrm{C}$.

\subsection{2}

\section{3'-Diaminobenzidine (DAB) immunohistochemistry}

3.3'-Diaminobenzidine (DAB) immunohistochemistry was performed on $4 \mu \mathrm{m}$ paraffin sections (section 2.4.1). First, sections were deparaffinized and rehydrated using the following treatments: 2 x 5 minutes in xylol (Roth, Karlsruhe, Germany); 10 minutes in $100 \% \mathrm{EtOH}(\mathrm{CVH}$, Hannover, Germany); 5 minutes in $95 \% \mathrm{EtOH} ; 5$ minutes in $70 \%$ $\mathrm{EtOH}$ and 1 minute in $\mathrm{dd}_{2} \mathrm{O}$. Then, sections were incubated for 30 minutes in a solution of $0.3 \% \mathrm{H}_{2} \mathrm{O}_{2}$ (Roth, Karlsruhe, Germany) in $0.01 \mathrm{M}$ PBS to block endogenous peroxidases. Antigen retrieval was achieved by heating the sections in $0.01 \mathrm{M}$ citrate buffer ( $\mathrm{pH}$ 6.0; Roth) for 10 minutes ( $800 \mathrm{~W}$ until boiling followed by $80 \mathrm{~W}$ for 8 minutes). After the sections were cooled down they were dipped for 1 minute in $\mathrm{dd}_{2} \mathrm{O}$ and permeabilized for 15 minutes in $0.1 \%$ Triton X-100 (Roth) in 0.01 M PBS. Sections were washed for 1 minute in 0.01 M PBS. To reveal intracellular $A \beta$, slides were incubated for 3 minutes in $88 \%$ formic acid (Roth). Two 5 minutes washing steps with $0.01 \mathrm{M}$ PBS were performed to remove any formic acid residues. Sections were circled with a lipid pen (Pap Pen; Kisker Biotech, Steinfurt, Germany) and incubated for 1 hour at RT with a solution of $10 \%$ fetal cow serum (FCS; Thermo Fisher Scientific, Waltham, MA, USA) and $4 \%$ lowfat dried milk powder (Roth) in $0.01 \mathrm{M}$ PBS to block unspecific antigens. Primary antibodies (TABLE 2.8) were diluted to the desired concentration in 0.01 PBS containing $10 \%$ FCS. After removing the blocking solution sections were incubated overnight at RT with $90 \mu \mathrm{l}$ of the diluted primary antibody.

On the second day of the staining, sections were washed three times for 5 minutes in $0.01 \mathrm{M}$ PBS with $0.1 \%$ Triton and briefly with $0.01 \mathrm{M}$ PBS. After washing, the sections were incubated for 1 hour at $37^{\circ} \mathrm{C}$ with the respective biotinylated secondary antibodies (TABLE 2.9). The secondary antibodies were diluted with 0.01 PBS containing $10 \%$ FCS to the desired concentration. Avidin-biotin complex (ABC) solution was prepared according the instructions of the manufacturer using the VECTASTAIN Elite ABC Kit (Vector Laboratories, Burlingame, CA, USA). Briefly, each component of the kit was added to a solution of $0.01 \mathrm{M}$ PBS containing $10 \%$ FCS at a dilution of $1: 100$. The ABC solution was incubated for $30{ }^{\circ} \mathrm{C}$ at $4{ }^{\circ} \mathrm{C}$ prior use. The sections were washed three times with $0.01 \mathrm{M}$ PBS before each section was incubated for 1.5 hour at $37^{\circ} \mathrm{C}$ with $90 \mu \mathrm{l}$ of $A B C$ solution. After incubation the slides were washed three times for 5 minutes with 0.01 $M$ PBS to remove the $A B C$ solution. Staining was visualized using $D A B$ as a chromagen. 
The DAB containing solution was prepared by adding $100 \mu \mathrm{l}$ of DAB stock solution (25 $\mathrm{mg} / \mathrm{ml}$ DAB in $50 \mathrm{mM}$ Tris/HCl; Sigma, St. Louis, MO, USA) and $2.5 \mu \mathrm{l} 30 \% \mathrm{H}_{2} \mathrm{O}_{2}$ to $5 \mathrm{ml}$ $50 \mathrm{mM}$ Tris/HCl (pH 7.5; Roth). Sections were incubated in $100 \mu \mathrm{LAB}$ solution until the desired staining pattern was seen. After staining with DAB the sections were washed 3 times for 5 minutes in 0.01 M PBS and counterstained for 40 seconds with hematoxylin (Roth). Sections were dipped in tab water and washed for 5 minutes under running tap water. After washing, sections were dehydrated using the following incubations: 1 minute in $70 \% \mathrm{EtOH} ; 5$ minutes in $95 \% \mathrm{EtOH} ; 10$ minutes in $100 \% \mathrm{EtOH}$ and $2 \times 5$ minutes in xylol. Stained slides were mounted with 2 drops of Roti®-Histokitt mounting medium (Roth) before a cover slip was added to each slide.

Bright field images of DAB-immunostained tissue were acquired using an BX-51 microscope equipped with a Camera (Olympus, Tokio, Germany). Figures were generated using Adobe Illustrator CS6 (Adobe Systems, San Jose, CA, USA).

\subsubsection{4'.6-diamidino-2-phenylindole (DAPI) staining}

4'.6-diamidino-2-phenylindole (DAPI) staining was performed on $4 \mu \mathrm{m}$ paraffin sections (section 2.4.1). First, sections were deparaffinized and rehydrated using the following treatments: 2 x 5 minutes in xylol (Roth, Karlsruhe, Germany); 10 minutes in $100 \% \mathrm{EtOH}$ ( $\mathrm{CVH}$, Hannover, Germany); 5 minutes in $95 \% \mathrm{EtOH} ; 5$ minutes in $70 \% \mathrm{EtOH}$; 1 minute in $\mathrm{ddH}_{2} \mathrm{O}$ and 1 minute in $0.01 \mathrm{M}$ PBS. DAPI staining was performed by dipping the sections for 1 minute in a solution of $1.5 \mathrm{mg} /$ DAPI (Sigma, St. Louis, MO, USA) dissolved in $\mathrm{dd}_{2} \mathrm{O}$. The slides were washed twice for 1 minute with $0.01 \mathrm{M}$ PBS. One drop of fluorescence mounting media (Dako, Glostrup, Denmark) was added to each section before a cover slip was applied.

Fluorescent images were acquired using an BX-51 microscope equipped with a camera (Olympus, Tokio, Germany). The mercury arc lamp and the filter cube set included in the BX51 system were used to generate pictures. Figures were generated using Adobe Illustrator CS6 (Adobe Systems, San Jose, CA, USA). 
The novel oligomeric $A \beta$ specific antibody NT4X-167 (lgG2b; official name of cell line Aß4-40 NT4X-167; DSM ACC3162) was generated by immunizing three Balb/c mice with unconjugated $A \beta_{4-40}$. In order to generate hybridoma cells the lymph nodes were prepared and fused with myeloma cell line P3-X63-Ag8 The hybridoma supernatants of mixed clones were screened by immunohistochemistry and enzyme-linked immune-absorbent assay (ELISA) and then subcloned. The hybridoma cells were screened by an ELISA for antibodies that bind $A \beta_{4-10}$ and $A \beta_{4-40}$, but not $A \beta_{36-40}$. Positive antibody clones were further screened by immunohistochemical staining of human brain sections. Finally, the monoclonal antibody NT4X-167 that recognizes the $\mathrm{N}$-terminus of $\mathrm{N}$-truncated $A \beta$ was identified. NT4X-167 binds most efficiently to $A \beta_{4-x}$ (Antonios et al., 2013).

Briefly, in order harvest the antibody hybridoma cells were routinely cultivated in 75 $\mathrm{cm}^{2}$ standard cell culture flaks (Greiner Bio-One, Kremsmuenster, Austria) and kept at 37 ${ }^{\circ} \mathrm{C}, 5 \% \mathrm{CO}_{2}$ in a humidified atmosphere (HERAcell $150 \mathrm{CO}_{2}$ Incubator, Thermo Fisher Scientific). Cells were cultivated in Opti-MEM reduced serum media (Life Technologies, Carlsbad, CA, USA) supplemented with $10 \%$ fetal calf serum (FCS, Biochrom, Cambridge, UK) and $100 \mathrm{U} / \mathrm{ml}$ Penicillin and $100 \mathrm{U} / \mathrm{ml}$ Streptomycin (PAN-Biotech, Aidenbach, Germany). Media was changed two times a week and cells were split every 10 days using Trypsin/EDTA (PAN Biotech). Cells were stepwise acclimated to $5 \%$ FCS, $3 \%$ FCS and finally $1 \%$ FCS media. After cells were acclimated in $1 \%$ FCS media, supernatant containing the antibody was collected and stored at $-20^{\circ} \mathrm{C}$ until further use.

\subsubsection{Primary Antibodies}

TABLE 2.8 Primary antibodies used for immunohistochemistry (IH) and Western Blots (WB)

\begin{tabular}{|c|c|c|c|c|c|c|}
\hline Antiserum & Host & Isotope & $\begin{array}{l}\text { Working } \\
\text { dilution }\end{array}$ & Immunogen & Source & Used for \\
\hline 24311 & rabbit & polyclonal & $1: 500$ & Pan-A $\beta$ & AG Bayer & $\mathrm{IH}, \mathrm{WB}$ \\
\hline $1-57$ & mouse & monoclonal & $1: 500$ & $A \beta_{p E 3-x}$ & $\begin{array}{l}\text { Synaptic } \\
\text { Systems }\end{array}$ & $\mathrm{IH}$ \\
\hline$A \beta 42$ & rabbit & polyclonal & $1: 500$ & $\begin{array}{c}\text { C-terminus } \\
\text { of } A \beta\end{array}$ & $\begin{array}{l}\text { Synaptic } \\
\text { Systems }\end{array}$ & $\mathrm{IH}$ \\
\hline GFAP & mouse & polyclonal & $1: 500$ & GFAP & Chemicon & $\mathrm{IH}$ \\
\hline IC16 & mouse & monoclonal & $1: 1,000$ & $A \beta_{1-x}$ & $\begin{array}{l}\text { generous gift } \\
\text { of } S . \text { Weggen }\end{array}$ & $\mathrm{IH}$ \\
\hline IBA1 & rabbit & polyclonal & $1: 500$ & IBA1 & Waco & $\mathrm{IH}$ \\
\hline
\end{tabular}




\subsection{3}

Secondary Antibodies

TABLE 2.9 Polyclonal secondary antibodies used for immunohistochemistry (IH) and Western Blots (WB horseradishperoxidase [HRP]-conjugated)

\begin{tabular}{llcccc}
\hline Antiserum & Host & Conjugate & $\begin{array}{c}\text { Working } \\
\text { dilution }\end{array}$ & Company & Used for \\
\hline anti-mouse, & rabbit & biotinylated & $1: 200$ & Dako & IH \\
anti-rabbit, & swine & biotinylated & $1: 200$ & Dako & IH \\
anti- rabbit & swine & HRP & $1: 3,000$ & Dianova & WB \\
\hline
\end{tabular}

2.6

Quantification of neuron numbers

2.6.1

Sample preparation

Frozen left brain hemispheres (section 2.1.5.2) were cut frontally into entire series of 30 $\mu \mathrm{m}$ thick sections on a CM1850 UV cryostat (Leica, Wetzlar, Germany). Every tenth section was systematically sampled, stained with cresyl violet (section 2.6.2) and used for stereological analysis (section 2.6.3).

\subsubsection{Cresyl violet staining}

Sections (section 2.6.1) were delipidated using the following treatments: $2 \times 10$ minutes in work solution $A$ followed by 20 minutes in work solution $B$ and $2 \times 10$ minutes in work solution $A$. Then the sections were stained twice for 10 minutes with cresyl violet staining solution. Sections were washed three times with work solution A to remove cresyl violet residues. After washing, sections were dehydrated using the following incubations: 3 x 10 minutes $100 \%$ ethanol (CVH, Hannover, Germany), 10 minutes isopropanol (Roth, Karlsruhe, Germany) and $2 \times 5$ minutes xylol (Roth). Stained slides were mounted with 2 drops of Roti®-Histokitt mounting medium (Roth) before a cover slip was added to each slide. During the whole staining process brain slides were covered with aluminum foil to avoid light exposure.

Work solution A: $13.61 \mathrm{~g}$ Natrium Acetate Trihydrate (Roth) was diluted in $100 \mathrm{ml} \mathrm{dd}_{2} \mathrm{O}$. $40 \mathrm{ml}$ of the generated $1 \mathrm{M}$ Natrium Acetate solution was mixed with $9.6 \mathrm{ml} 100 \%$ Acetic Acid (Merck, Darmstadt, Germany). The volume was adjusted to 1 I with $\mathrm{dd}_{2} \mathrm{O}$. 


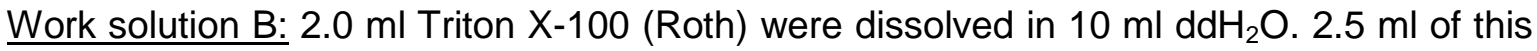

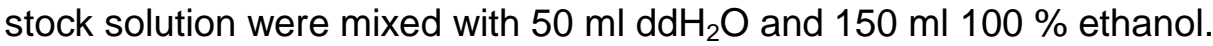

Staining solution: $0.1 \mathrm{~g}$ cresyl violet (Fluka, St. Louis, MO, USA) was added to 1 I work solution $A$ and stirred for 1 hour.

\subsubsection{Microscopy and quantification of neurons}

Design-based stereology was performed on cresyl violet stained brain sections (section 2.6.2) to quantify the neuron numbers in the CA1 of the hippocampus in an unbiased way. Therefore a BX51 stereology workstation (Olympus, Shinjuku, Japan) with a motorized specimen stage for automatic sampling and Stereo Investigator 7 software (MBF Bioscience, Williston, VT, USA) were used.

A

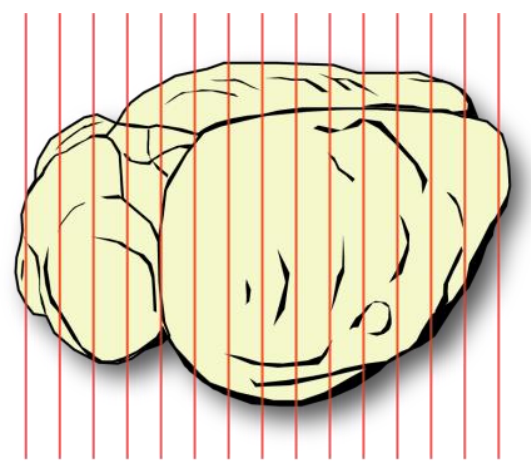

B

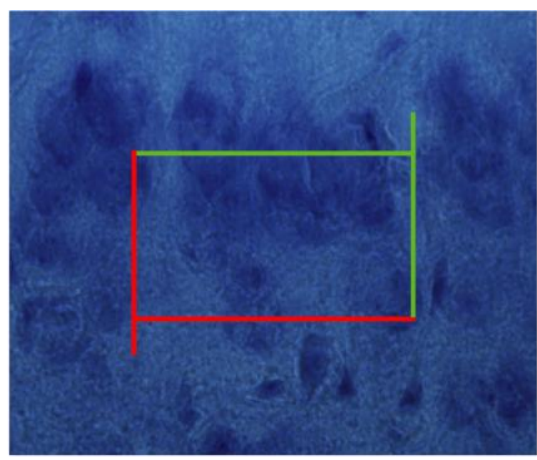

C
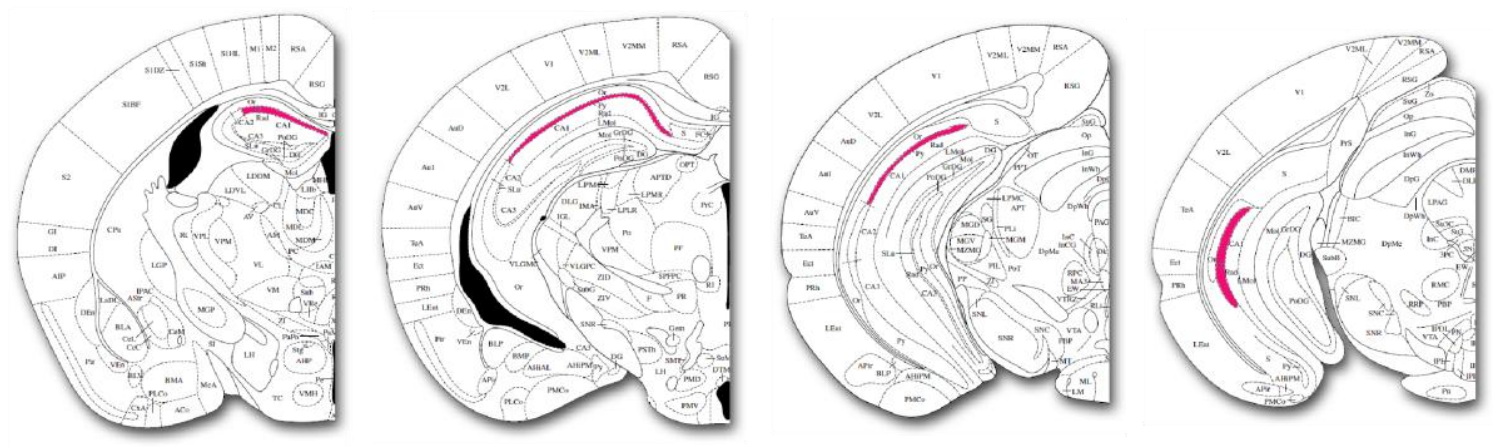

FIGURE 2.5 Procedure of design-based stereology of the CA1. (A) Brain hemispheres are frontally cut and sampled at a defined frequency (every 10th section). (B) The number of cells are counted under high magnification. Cells that are in the box or partially within but touching the green line are counted. All cells that are outside the box or touch the red line are excluded from counting. Counting areas in the CA1 are selected by the software in a systematic random manner. (C) Coronal slices of mouse brain (adapted from Franklin and Paxinos, 2012). CA1 is outlined from left to right: Bregma $-1.22,-2.46,-3.08$ and $-3.80 \mathrm{~mm}$.

The hippocampal pyramidal cell layer CA1 (Bregma -1.22 to $-3.80 \mathrm{~mm}$ ) (FIGURE 2.5 C) were delineated at low magnification $(4 \mathrm{x})$ and cell counts were performed at high 
magnification (100x) (Franklin and Paxinos, 2012). A $14 \mu \mathrm{m} \times 14 \mu \mathrm{m}$ counting frame was applied within a $49 \mu \mathrm{m} \times 105 \mu \mathrm{m}$ sampling grid to count neurons in the CA1. Neuronal nuclei were sampled randomly using optical disector probes and the total number of neurons was subsequently estimated by the optical fractionator method using a $2 \mu \mathrm{m}$ top guard zone (West et al., 1991). Section thickness was measured on every grid site with a $5 \mu \mathrm{m}$ dissector height (Z). Samples were blinded to avoid bias counting. Neurons were counted according to the principles of stereology (West et al., 1991; Schmitz and Hof, 2005) and the total number of neurons (N) was calculated by formula (1) and (2). The remainig stereological parameters are explained in TABLE 2.10 and the parameters for counting the CA1 are given in TABLE 2.11.

$$
P=\operatorname{asf} \times s s f \times t s f
$$

(2)

$$
N=\sum_{i=1}^{n}(P \times Q) i
$$

TABLE 2.10 Definition of stereological parameters

\begin{tabular}{ll}
\hline \multicolumn{2}{l}{ Stereological parameters } \\
\hline asf & area sampling fraction $(\mathrm{xy} / \mathrm{XY})$ \\
ssf & section sampling fraction \\
tsf & thickness sampling fraction $(\mathrm{T} / \mathrm{Z})$ \\
$\mathrm{Z}$ & dissector height \\
$\mathrm{P}$ & number of neurons \\
$\mathrm{T}$ & mean section thickness \\
$\mathrm{Q}$ & total markers counted (neuron number)
\end{tabular}


TABLE 2.11 Parameters for stereological analysis of neurons in the CA1

\begin{tabular}{lc}
\hline Parameter & CA1 \\
\hline Sampling Grid $(\mathrm{x})(\mu \mathrm{m})$ & 49 \\
Sampling Grid $(\mathrm{y})(\mu \mathrm{m})$ & 105 \\
Sampling Grid Area $(\mathrm{xy})\left(\mu \mathrm{m}^{2}\right)$ & 5145 \\
Counting Frame Width $(\mathrm{X})(\mu \mathrm{m})$ & 14 \\
Counting Frame Height $(\mathrm{Y})(\mu \mathrm{m})$ & 14 \\
Counting Frame Area $(\mathrm{XY})\left(\mu \mathrm{m}^{2}\right)$ & 196 \\
asf & 26,25 \\
ssf & 10 \\
$\mathrm{~T}(\mu \mathrm{m})$ [average value] & 9,6 \\
$\mathrm{Z}(\mu \mathrm{m})$ & 5 \\
\hline
\end{tabular}

\subsection{Deep Sequencing of mouse brains}

\subsubsection{RNA expression profiling}

RNA expression profiling was performed by next-generation sequencing on a SOLiD 5500xI Genetic Analyzer (Life Technologies, Carlsbad, CA, USA). RNA was extracted from frozen mouse brain hemispheres (section 2.1.4) as follows. The tissue was homogenized using a Polytron (VWR, Radnor, PA, USA) device and then treated with TRIzol (Life Technologies, Carlsbad, CA, USA). Next, $5 \mu \mathrm{g}$ of each total RNA sample were spiked with ERCC spike-in control mixes (Life Technologies, Carlsbad, CA, USA) before removal of the rRNA by use of a RiboZero Kit (Epicentre, Madison, WI, USA). The RNA was prepared for sequencing following the protocol provided by the manufacturer of the sequencer. In brief, the rRNA depleted RNA was fragmented by chemical hydrolysis, phosphorylated and purified. Adaptors were ligated to the RNA fragments which subsequently were reverse transcribed into cDNA. The cDNA was purified and sizeselected using two rounds of Agencourt AMPure XP bead purification (Beckman Coulters Genomics, Danvers, USA) and released from the beads. The sample was amplified by 12 PCR cycles in the presence of primers that contained unique sequences (barcoding). The size distribution and concentration of the fragments were determined with an Agilent 2100 Bioanalyzer (Agilent Technologies, Santa Clara, USA) and the corresponding chemicals (Agilent Technologies). 
The cDNA fragments were pooled in equimolar amounts and diluted to $76 \mathrm{pg} / \mu \mathrm{l}$ corresponding to a concentration of $500 \mathrm{pM} .50 \mu \mathrm{l}$ of this dilution was mixed with a freshly prepared oil emulsion, P1 and P2 reagents and P1 beads in a SOLiD EZ Bead Emulsifier (Life Technologies) were prepared according to the E80 scale protocol (Life Technologies). The emulsion PCR was carried out in a SOLiD EZ Bead Amplifier (Life Technologies) using the E80 setting. To enrich for the beads that carried amplified template DNA, the beads were purified on a SOLiD EZ Bead Enricher using the recommended chemicals and software (Life Technologies). The purified beads were loaded onto a SOLiD 6-lane Flowchip (Life Technologies) and incubated upside down for 1 hour at $37^{\circ} \mathrm{C}$. The Flowchip was positioned in the 5500xI SOLiD System and the DNA was sequenced using the settings and recommended chemicals for sequencing 75 nucleotides in the forward direction and 35 nucleotides in the reverse direction (Life Technologies).

Sequence reads were mapped to the mouse genome reference sequence $\mathrm{mm} 10^{1}$ using the workflow 'whole.transcriptome.pe' of analysis software LifeScope-v2.5.1-r0 (Life Technologies). Reads mapping to RefSeq coding exons (accessed 2012-06-27) ${ }^{2}$ and matching the coding strand were considered coding RNAs. All other mapping reads were considered non-coding. RNA expression profiling of mouse brain tissue was carried out by the group of Prof. Andreas W. Kuss at the Ernst-Moritz-Arndt University Greifswald, Germany.

\subsection{2}

\section{Differential Expression Analysis}

RNASeq read data were normalized within and between lanes for GC-content using EDASeq's full-quantile normalization (Risso et al., 2011). The differential expression analysis was done with DESeq (Anders and Huber, 2010). All samples were treated as replicates of a single condition for the estimation of the dispersion. Only the fitted dispersion values were used in the following analyses. The significance of differential expression was determined by the Benjamini-Hochberg corrected $p$-values of the negative binomial test between two conditions. The threshold for significance was set to $p=0.05$. Differential expression analysis was carried out by Tim Kacprowski of the group of Mario Albrecht at the University Medicine Greifswald, Germany.

\footnotetext{
${ }_{1}^{1} \mathrm{http}: / /$ hgdownload.cse.ucsc.edu/goldenPath/mm10

${ }^{2}$ http://hgdownload.cse.ucsc.edu/goldenPath/mm10/database/refGene.txt.gz.
} 


\subsection{3}

\section{Annotation analysis}

In order to gain insight in the biological function and to understand the biological significance of differentially expressed genes (DEGs), the functional annotation of DEGs was obtained using Source ${ }^{3}$, GeneCards ${ }^{4},{\text { Wiki- } \mathrm{Pi}^{5} \text { and Mouse Genome Informatics }}^{6}$ as well as the literature.

\section{8}

\section{Statistical analysis}

Details of statistical analysis, methods and parameters are given in the respective results sections where data is described. The number of biological replicates $(n)$ is given at the base of figures where necessary. Significance levels are given as follows: ${ }^{* \star *} p<0.001$; ${ }^{* *} p<0.01 ;{ }^{*} p<0.05$. Unless stated otherwise, data are given as means \pm standard error of the mean (SEM).

All statistics for behavior experiments were calculated using GraphPad Prism version 5.04 for Windows (GraphPad Software, San Diego, CA, USA) and Statistica version 12.0 (StatSoft, Tulsa, OK, USA). Differences between groups were tested with unpaired t-test, paired t-test, one-way analysis of variance (ANOVA) followed by Bonferroni multiple comparison or two-way ANOVA followed by Bonferroni multiple comparison as indicated in the results part.

Statistical analysis of quantitative RT-PCR measurements was performed using the Relative Expression Software Tool V1.9.6 (REST, Qiagen, Hilden, Germany) (Pfaffl et al., 2002). The expression ratio results of the studied transcripts were tested for significance by Pair Wise Fixed Reallocation Randomization Test. Statistical analysis of deep sequencing data was performed as described in the respective section (section 2.7.2).

\footnotetext{
${ }^{3} \mathrm{http}: / /$ puma.princeton.edu/cgi-bin/source/sourceResult

${ }^{4} \mathrm{http}: / / \mathrm{www}$.genecards.org

${ }^{5} \mathrm{http}: / /$ severus.dbmi.pitt.edu/wiki-pi/index.php

${ }^{6} \mathrm{http}: / /$ www.informatics.jax.org
} 
2.9

Software

\begin{tabular}{ll}
\hline Software & Source \\
\hline Adobe Illustrator CS6 & Adobe Systems, San Jose, CA, USA \\
Microsoft Office 2007 & Microsoft, Redmond, WA, USA \\
PyMOL V1.7.2 & Schrödinger, New York, NY, USA \\
Relative Expression Software Tool V1.9.6 & Qiagen, Hilden, Germany \\
GraphPad Prism V5.04 & GraphPad Software, San Diego, CA, USA \\
Statistica version 12.0 & StatSoft, Tulsa, OK, USA
\end{tabular}

All Figures without a reference are own figures created with Adobe Illustrator, PyMOL and GraphPad Prism. Protein data bank ${ }^{7}$ codes 1IYT, 1MWP, 1OWT, 2ROZ, 2YT0, 3DXC and $3 N Y L$ were used for figure generation.

${ }^{7}$ www.rcsb.org 
$A \beta_{4-42}$ represents a major $N$-truncated $A \beta$ isoform in the cortex and hippocampus of $A D$ patients (Portelius et al., 2010). However, little is known about the role of this peptide to the progression of $A D$. In order to investigate the consequences of chronic expose of $A \beta_{4}$ 42 in vivo the transgenic mouse model Tg4-42 was generated (Wittnam, 2012). The main objectives of this part of the project were the generation of homozygous Tg4-42 mice (Tg4-42 hom) and the characterization of Tg4-42 and Tg4-42 hom mice. Among others, the amyloid pathology in these mice was examined and the behavior phenotype analyzed. Furthermore, the question was addressed whether $A \beta_{4-42}$ or $A \beta_{p E 3-42}$ may precede the other in AD pathology in the 5XFAD mouse model using the newly generated antibody NT4X-167.

\subsection{1}

$A \beta_{4-42}$ elicits stable aggregates similar to $A \beta_{1-42}$ and $A \beta_{p E 3-42}$

The aggregation properties of freshly prepared and aged $A \beta_{4-42}$ were compared with $A \beta_{1-42}, A \beta_{p E 3-42}, A \beta_{4-38}, A \beta_{4-40}$ peptides. Therefore, peptides were subjected to SDS-PAGE Western Blot (FIGURE 3.1). Under denaturing conditions freshly dissolved $A \beta_{1-42}, A \beta_{p E 3-42}$ and $A \beta_{4-42}$ displayed monomers, dimers and aggregates most likely presenting trimers or tetramers, while $A \beta_{4-38}$ and $A \beta_{4-40}$ formed monomers and dimers but no other oligomers. After 24 and 72 hours of aging $A \beta_{1-42}, A \beta_{p E 3-42}$ and $A \beta_{4-42}$ peptides showed in addition higher molecular weight oligomers. In contrast, aged $A \beta_{4-38}$ and $A \beta_{4-40}$ retrained their pattern and did not exhibit additional higher molecular weight aggregates. Aged and fresh $A \beta_{4-42}$ showed a similar aggregation pattern as $A \beta_{1-42}$ and $A \beta_{p E 3-42}$. 


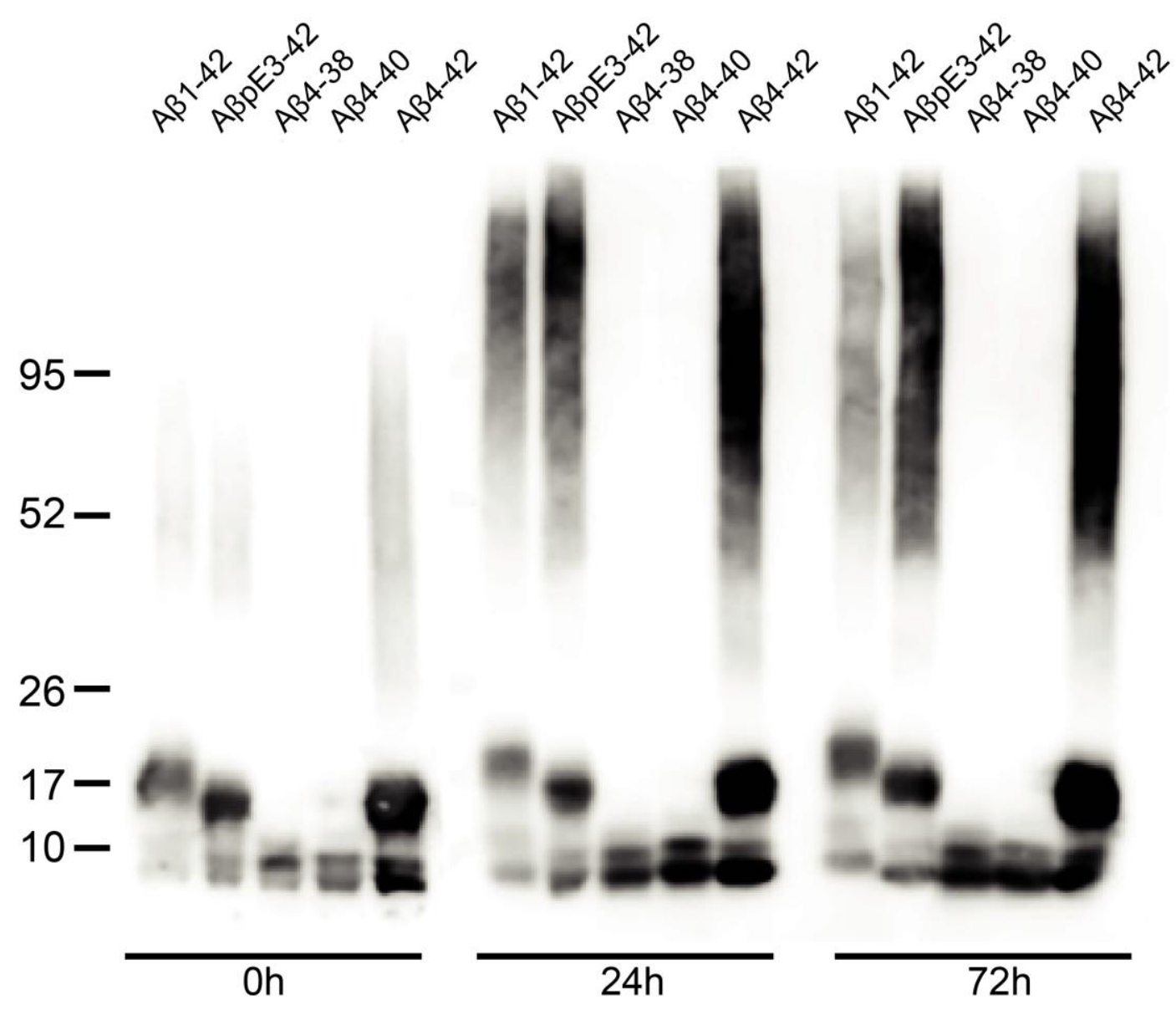

FIGURE 3.1 Freshly dissolved $A \beta_{1-42}, A \beta_{\text {pE3-42, }} A \beta_{4-38}, A \beta_{4-40}$ and $A \beta_{4-42}$ rapidly formed stable aggregates. All peptides displayed dimeric oligomers and monomers under reducing conditions. Furthermore $A \beta_{1-42}, A \beta_{P E 3-42}$ and $A \beta_{4-42}$ developed SDS-stable tri- or tetrameric oligomers. Aged $A \beta_{1-42}, A \beta_{p E 3-42}$ and $A \beta_{4-42}$ peptides retained this pattern and exhibited also higher molecular weight aggregates. SDS-PAGE Western blot of $A \beta$ peptides using the polyclonal antiserum 24311.

\subsection{2}

\section{Early intraneuronal $A \beta_{4-x}$ accumulation in 5XFAD transgenic mice}

$N$-truncated $A \beta_{4-42}$ is next to $A \beta_{\text {pE3-42 }}$ the major $N$-truncated $A \beta$ variant in the $A D$ brain (Portelius et al., 2010). In order to determine whether $A \beta_{4-42}$ or $A \beta_{p E 3-42}$ precedes the other in the AD pathology 5XFAD mice were analyzed with the new NT4X-167 antibody. Cortical sections of hemizygous and homozygous 5XFAD mice were studied as this brain area is known for abundant intraneuronal $A \beta$ (Oakley et al., 2006; Antonios et al., 2013). The staining pattern of NT4X-167 (against $A \beta_{4-x}$ and pyroglutamate $A \beta_{3-x}$ ) was compared with 1-57 (against $A \beta_{p E 3-x}$ ) and IC16 (against $A \beta_{1-x}$ ). Homozygous 5XFAD mice were used in order to observe an aggravated amyloid pathology at an earlier time point as compared to hemizygous mice (Antonios et al., 2013). As expected, intraneuronal $A \beta_{1-x}$ accumulation was observed in young (FIGURE 3.2 A and B), but not in aged 5XFAD mice (FIGURE 3.2 
C). Homozygous 6 week-old 5XFAD mice showed an higher intraneuronal signal compared to hemizygous mice (FIGURE 3.2 A vs. B). Aged mice demonstrated abundant $A \beta_{1-x}$ accumulation in amyloid plaques (FIGURE $3.2 \mathrm{C}$ ). NT4X-167 recognized intraneuronal $A \beta$ in 6 week-old homozygous 5XFAD mice (FIGURE 3.2 E). Intraneuronal $A \beta$ could not be detected with the $A \beta_{p E 3-x}$ specific antibody 1-57 in 6 week-old homozygous 5XFAD (FIGURE 3.2 H). Therefore the signal was due to $A \beta_{4-x}$ accumulation, which represents the earliest $N$-truncated $A \beta$ species. Using the 1-57 antibody no intraneuronal signal could be detected at any ages analyzed. Amyloid plaques were detected with both NT4X-167 and 1-57 in aged mice (FIGURE 3.2 F and I).
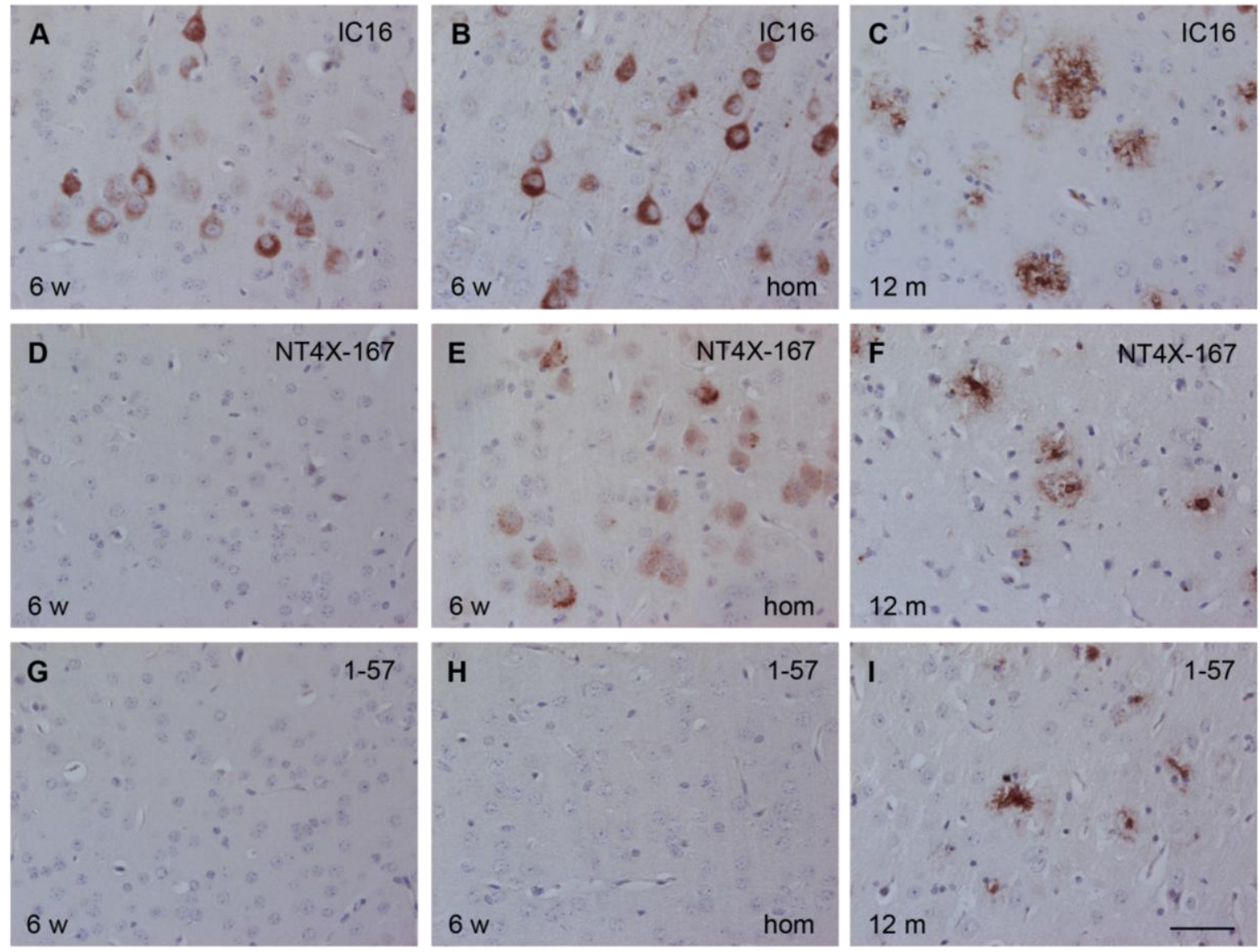

FIGURE 3.2 Immunohistochemical staining of cortical sections of 5XFAD transgenic mice. (A) Immunostaining with IC16 demonstrating intraneuronal $A \beta$ accumulation in 6 week old hemizygous 5XFAD. (B) Homozygous 5XFAD mice exhibited more intensive intraneuronal staining at the same age. (C) Abundant extracellular plaque staining with IC16 at the age of 12 months. (D) No signal was detected using NT4X-167 in 6 week old hemizygous 5XFAD. (E) In homozygous 5XFAD mice significant intraneuronal staining was observed with NT4X-167. (F) Abundant extracellular plaque staining with NT4X-167 at the age of 12 months. (G) No intraneuronal A $\beta$ was observed with the pyroglutamate specific antibody 1-57 in 6 week-old hemizygous and $(H)$ homozygous 5XFAD mice. (I) Extracellular plaque staining with 1-57 at the age of 12 months. At 12 months only hemizygous 5XFAD were used. Abbreviations: $w=$ week; $m=$ month. Scale bar in I for $50 \mu \mathrm{m}$. 


\subsection{3}

Characterization of $\mathrm{Tg} 4-42$ mice

The Tg4-42 mouse model represents the first mouse model that exclusively expresses $A \beta_{4-42}$ under the control of the Thy-1 promoter without additional overexpression of mutant PSEN-1 or APP. Tg4-42 mice neuronally express human $A \beta_{4-42}$ fused to the murine thyrotropin releasing hormone $(T R H)$ signal peptide. The fusion peptide routes $A \beta_{4-42}$ through the secretory pathway facilitating its extracellular release (Wirths et al., 2010; Alexandru et al., 2011). In order to reach its final form, the $A \beta_{4-42}$ produced in Tg4-42 mice does not need to go through additional enzymatic modification with the exception of the removal of the TRH signal peptide (Wittnam, 2012).

\subsubsection{1}

Generation of homozygous Tg4-42 mice

Hemizygous Tg4-42 mice expressing human $A \beta_{4-42}$ were previously generated in our lab (Wittnam, 2012; Bouter et al., 2014). In addition, homozygous Tg4-42 mice were bred and named Tg4-42 hom. Therefore, hemizygous mice were crossed to produce potential homozygous mice. Potential homozygous mice were identified by qRT-PCR (FIGURE 3.3) and backcrossed with wild-type mice to test for true homozygosity. Once homozygous males and females were confirmed, they were re-mated to each other as the founders for the new homozygous transgenic line Tg4-42 hom.

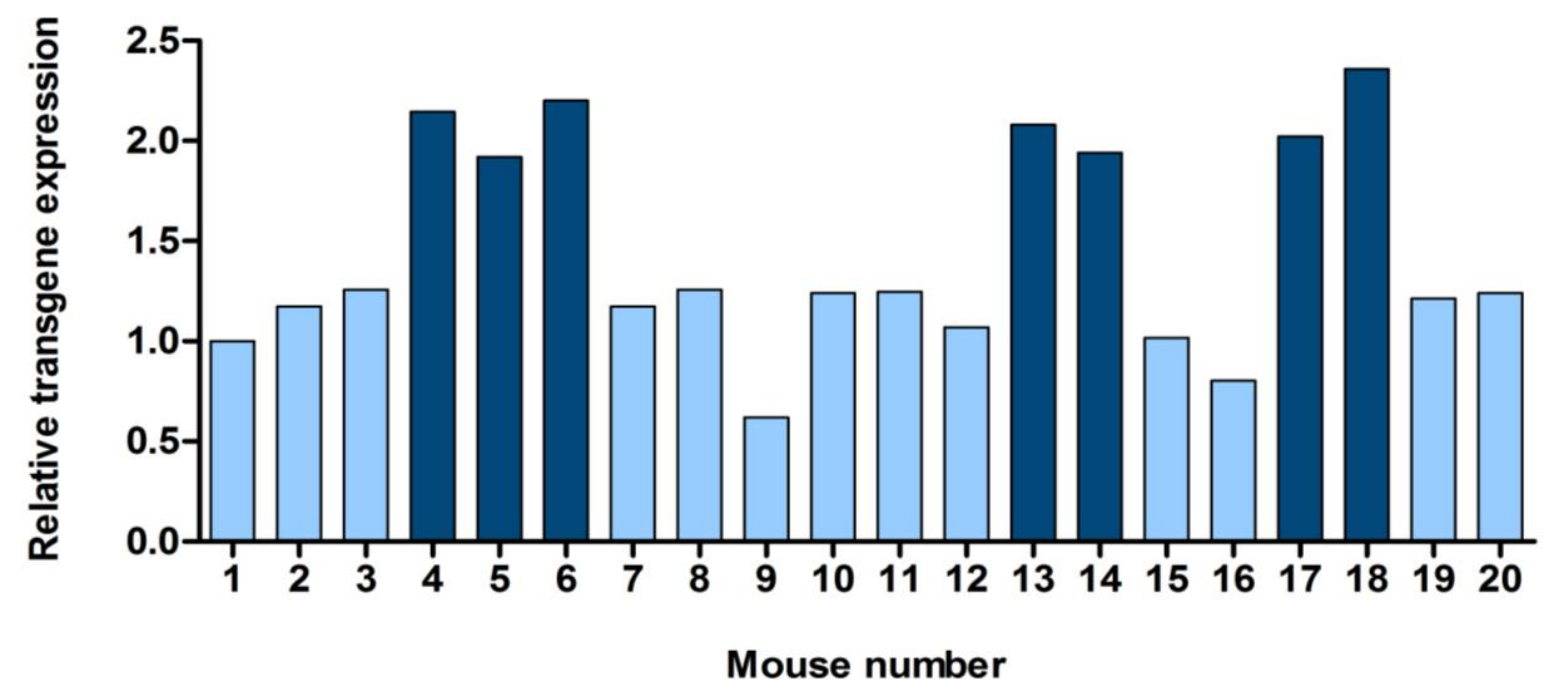

FIGURE 3.3 Identification of potentially homozygous Tg4-42 mice through qRT-PCR. All animals were compared to the hemizygous mouse number 1 . Transgene expression was quantified relative to APP. Mice number 4, 5, 6 and 13 were identified as potential homozygous males and 14,17 and 18 were identified as potential homozygous females. All other mice were considered as hemizygous. 


\subsubsection{2}

Amyloid beta expression in Tg4-42 mice

Transgene expression in $\mathrm{Tg} 4-42$ and $\mathrm{Tg} 4-42_{\text {hom }}$ mice was analyzed using immunohistochemistry.
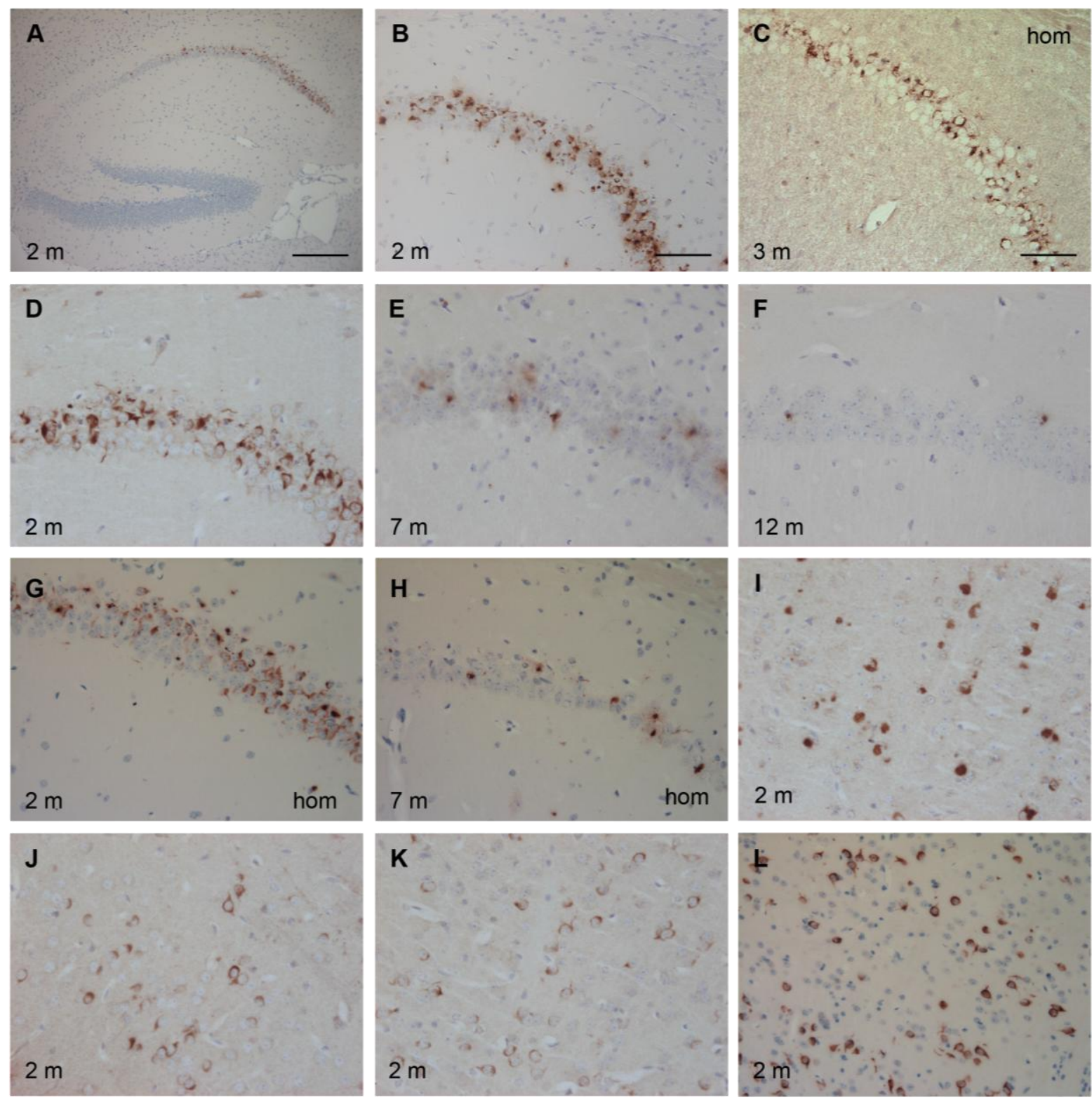

FIGURE 3.4 Immunohistochemical staining profile of Tg4-42 mice. Abundant intraneuronal A $\beta$ immunoreactivity was observed Tg4-42 mice in the CA1 pyramidal cell layer of the hippocampus starting at $(A, B, D) 2$ months of age. (D-F) $A \beta_{42}$ immunostaining declined with age. (G) $A \beta$ accumulation was also detected intraneuronally in the CA1 of Tg4-42 hom starting at 2 months. (G$\mathrm{H}) \mathrm{A} \beta$ staining decreased with age in Tg4-42 hom. Next to the (D-H) $A \beta_{42}$-specific antibody $A \beta 42$, the (A-B) polyclonal antiserum 24311 and (C) $A \beta 4-x$ binding antibody NT4X-167 detected $A \beta$ accumulation in the CA1. Other brain regions with $A \beta_{42}$ staining were (I) occipital cortex, (J) piriform cortex, $(K)$ striatum and (L) superior colliculus of 2-month-old Tg4-42 mice. Antibody: (AB) polyclonal antiserum 24311; (C) NT4X-167; (D-L): A 42 . Abbreviations: $m=$ months, hom = homozygous. Scale bar: (A) $200 \mu \mathrm{m}$; (B): $100 \mu \mathrm{m}$; (C-L): $50 \mu \mathrm{m}$. 
$A \beta$ expression in Tg4-42 mice was detected with the polyclonal antiserum 24311 recognizing pan-A $\beta$ and the $A \beta_{42}$-specific antibody $A \beta 42$. Furthermore, the novel monoclonal antibody NT4X-167, that recognizes the $\mathrm{N}$-terminus of $\mathrm{N}$-truncated $A \beta$ was used to analyze the transgene expression (Antonios et al., 2013).

Tg4-42 and $\operatorname{Tg} 4-42_{\text {hom }}$ mice brain sections showed strong intraneuronal $A \beta$ immunoreactivity predominantly in the CA1 region of the hippocampus beginning at two months of age (FIGURE 3.4 A, B, D and G). No difference in $A \beta$ expression was observed between the polyclonal antiserum 24311, the $A \beta_{42}$-specific antiserum $A \beta 42$ and the monoclonal NT4X-167 antibody (FIGURE $3.4 B$ and D). $A \beta$ expression in the CA1 pyramidal cell layer of the hippocampus declined during aging in Tg4-42 (FIGURE 3.4 DF) and Tg4-42 hom mice (FIGURE 3.4 G-I).

Intraneuronal $A \beta$ staining was also observed in Tg4-42 mice in the occipital cortex (FIGURE $3.4 \mathrm{I}$ ), piriform cortex (FIGURE $3.4 \mathrm{~J}$ ), striatum (FIGURE $3.4 \mathrm{~K}$ ) and superior colliculus (FIGURE $3.4 \mathrm{~L}$ ) starting at two months of age.

\subsubsection{Gliosis in Tg4-42 mice}

DAB immunohistochemistry was used to analyze gliosis in Tg4-42 mice (FIGURE 3.5).
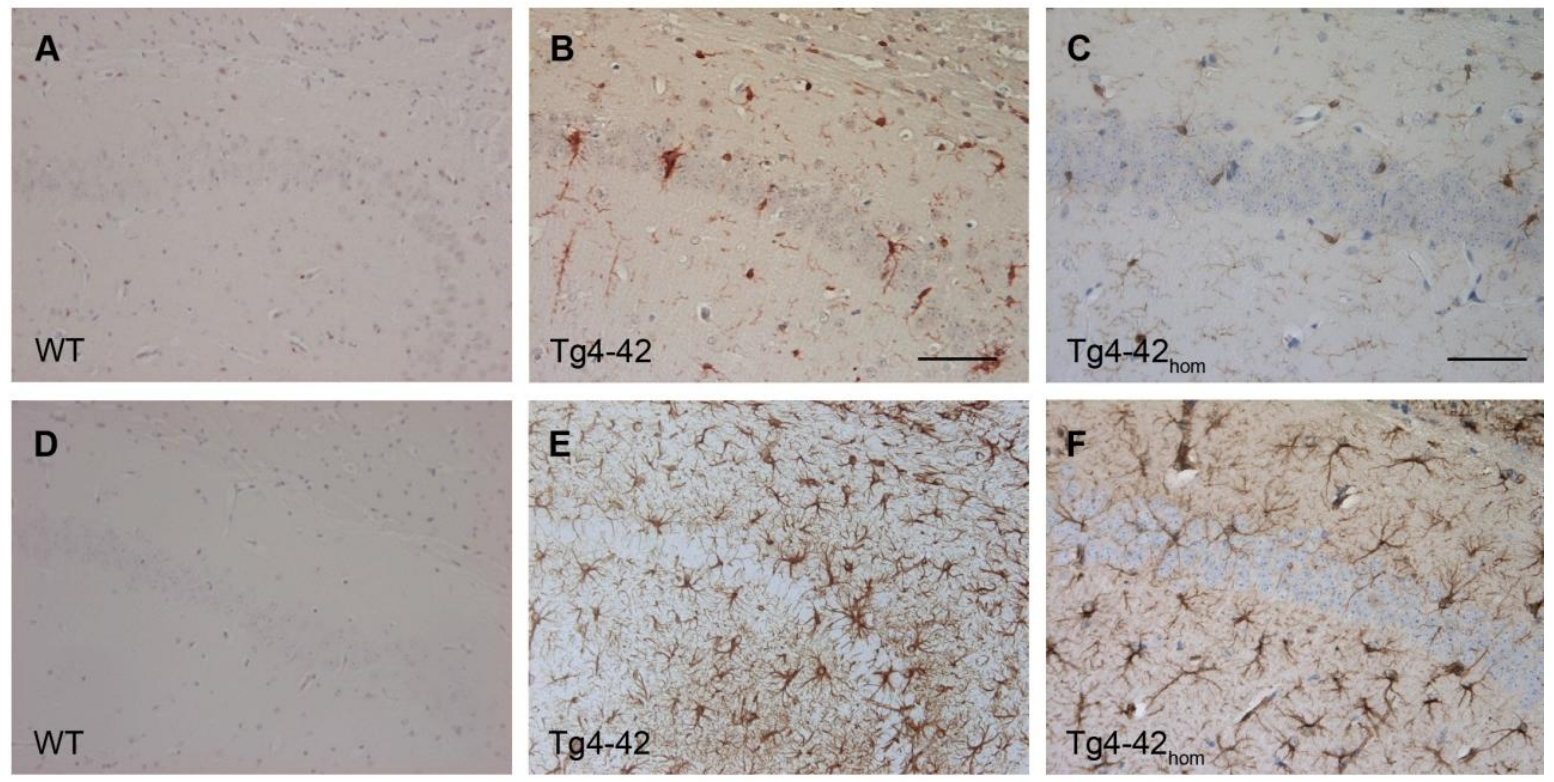

FIGURE 3.5 Reactive microglia and astroglia in Tg4-42 mice. (A-C) Microgliosis with IBA1 staining was detected at 2 months in (B) hemizygous Tg4-42 and (C) homozygous Tg4-42 mice. (D-F) Increased astrogliosis with GFAP staining was seen as early as 2 months of age in (E) hemizygous Tg4-42 and (F) homozygous Tg4-42 mice. No significant astro- and microgliosis were observed in wildtype (WT) controls (IBA1 A; GFAP D). Scale bar: (A-B, D-E) $100 \mu \mathrm{m}$; (C, F) $50 \mu \mathrm{m}$. 
Astrogliosis was examined with GFAP (glial fibrillary acidic protein) staining, while microgliosis was examined with IBA1 (ionized calcium binding adaptor molecule 1) staining. Reactive microglia were seen in the $\mathrm{CA} 1$ region of the hippocampus beginning at 2 months of age in Tg4-42 and Tg4-42 hom mice (FIGURE 3.5 B-C). Furthermore, visible increased astrogliosis was observed around the hippocampus in transgenic mice as early as two months (FIGURE 3.5 E-F). No significant astro- and microgliosis were detected in same-aged wild-type control mice (FIGURE 3.5).

\subsubsection{Physical and behavioral characterization of Tg4-42 mice}

The progressive memory decline is probably the most striking sign in AD patients (Alzheimer's Association, 2012). Therefore, a valid AD mouse model should reflect some of the behavioral changes observed in $A D$ patients. In order to determine if the $A \beta$ pathology in Tg4-42 mice was sufficient to induce functional impairments, cohorts of 3-, 8and 12-month-old Tg4-42 mice were subjected to a basic physical examination and a battery of behavioral tests. Furthermore, 3- and 8-month-old Tg4-42 hom were analyzed. All analyzed groups were sex-matched.

\subsection{General physical assessment of Tg4-42 mice}

Tg4-42 mice were subjected to a basic physical assessment to determine potential impairments caused by the $A \beta$ pathology. The physical examination revealed no abnormalities in Tg4-42 and Tg4-42 clasping phenotype during the tail suspension task regardless of sex and age. Transgenic mice tried to escape the grip by kicking their paws and twisting their whole body similar to healthy wild-type mice.

In order to determine possible effects on the physiological development, the body weight of Tg4-42 and wild-type mice was measured at three, eight and twelve months of age. Furthermore, the body weight of $\mathrm{Tg} 4-42_{\text {hom }}$ was measured at three and eight months of age. The sex distribution was balanced in all cohorts ( $n=12$ - 15 mice per group). Tg4-42 and Tg4-42 hom demonstrated no altered body weight compared to wild-type mice at all tested time points (FIGURE 3.6). Two-way repeated measures ANOVA revealed that there was no significant main effect of genotype (genotype: $p=0,064207$ ). Furthermore, all groups showed a comparable age-dependant weight gain (age: $p=0,000001$ ). 


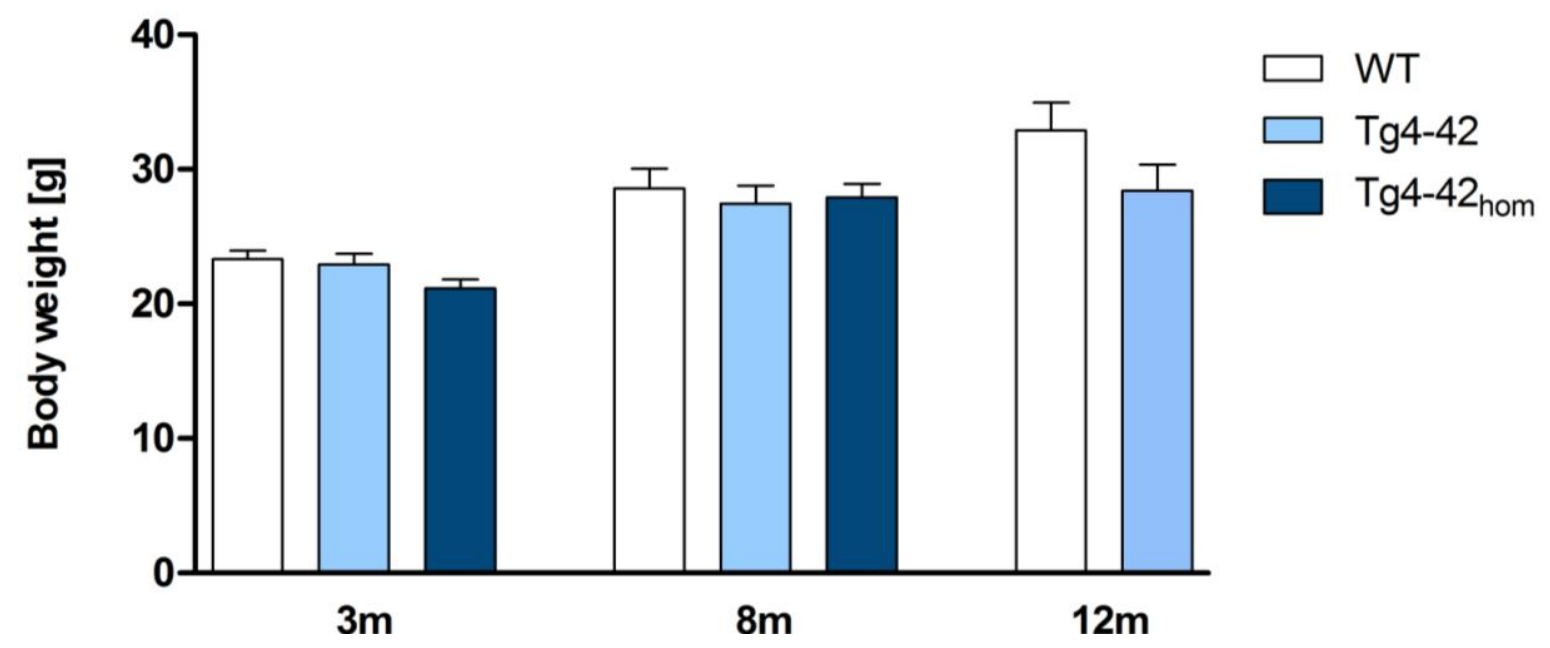

FIGURE 3.6 Body weight of Tg4-42 mice. 3-, 8- and 12-month-old Tg4-42 as well as 3- and 8-month-old Tg4-42 hom mice displayed normal body weight compared to aged-matched WT mice. Two-way repeated measures ANOVA, $n=12-15$ mice per group (equal sex distribution); data presented as mean \pm S.E.M; $m=$ months.

\subsection{Intact motor function in Tg4-42 mice}

Motor function in Tg4-42 mice was examined using the balance beam (FIGURE 3.7 B) and string suspension task (FIGURE 3.7 D). The balance beam task assesses fine motor coordinating and balance of mice (Hau and Schapiro, 2002; Luong et al., 2011). Grip and muscle strength as well as motor coordination of mice can be tested in the string suspension task (Hullmann, 2012; Arendash et al., 2001b). No significant difference between transgenic and wild-type control mice was detected at three, eight or twelve months of age in either motor test (FIGURE 3.7 A and C). Two-way repeated measures ANOVA revealed that there was no significant main effect of genotype (genotype: balance beam: $p=0,184313$; string suspension: $p=0,084051$ ) and Tg4-42 and Tg4-42 hom performed equally to same-aged wild-type control mice. Furthermore, performance in both tests declined with age independent of the genotype (two-way repeated measures ANOVA, age: balance beam: $p=0,000264$; string suspension: $p=0,008633)$. In summary, it can be stated that Tg4-42 and Tg4-42 hom mice showed no motor deficits. 


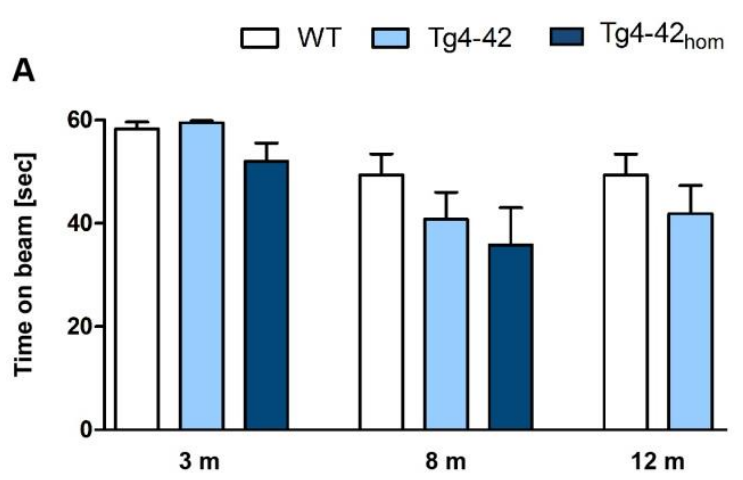

B

C
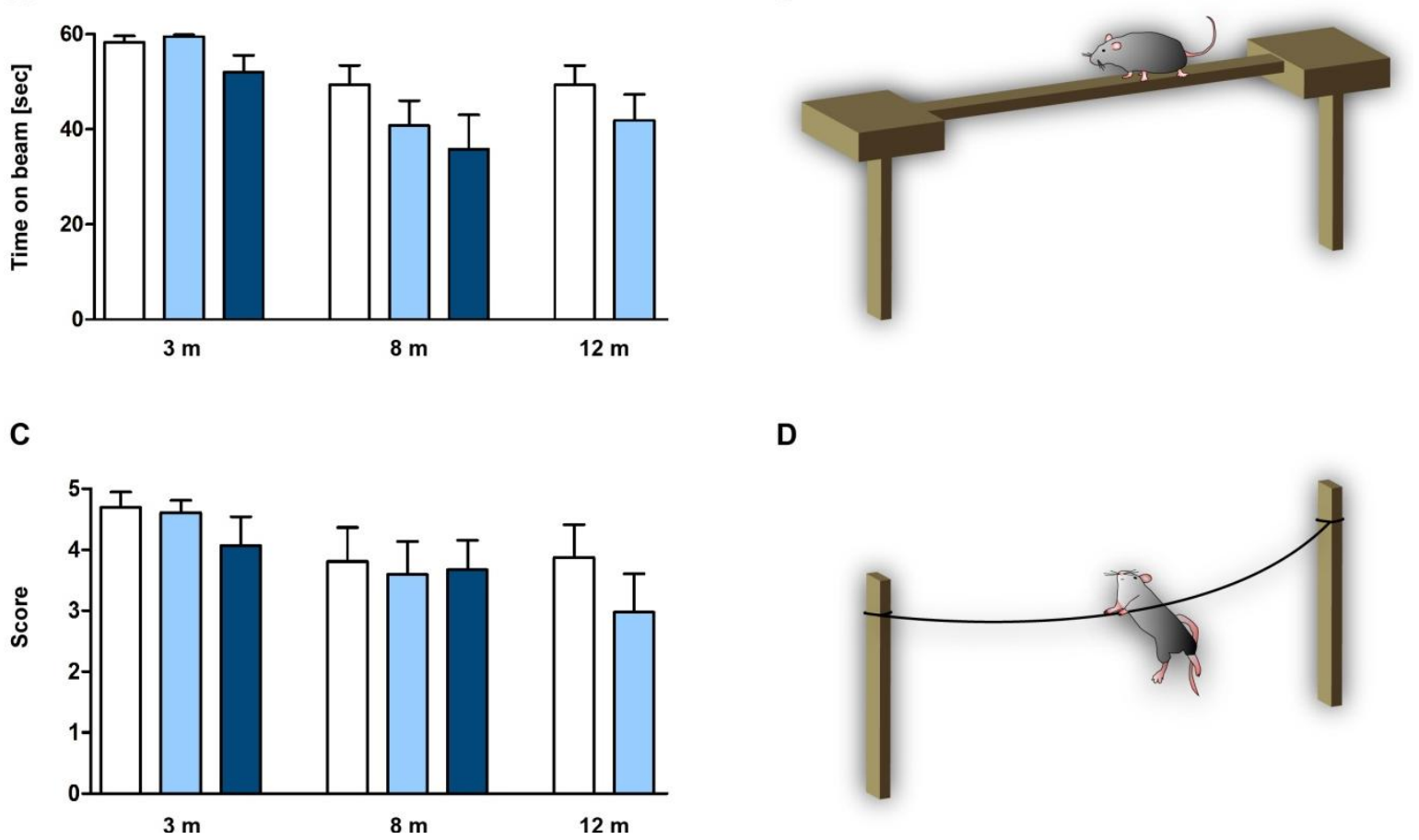

D

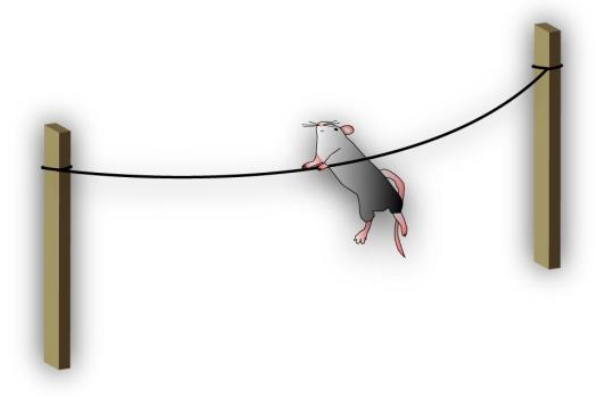

FIGURE 3.7 No motor deficits in Tg4-42 mice. Tg4-42 and Tg4-42 hom showed no deficits in the (A, $B)$ balance beam and the (C, D) string suspension task. 3, 8 and 12 months old Tg4-42 mice performed comparably to same-aged wild-type mice. Furthermore, no significant difference was detected between 3 and 8 months old Tg4-42 hom and wild-type controls. Two-way repeated measures ANOVA, $n=10-14$ per group; data presented as mean \pm S.E.M; $m=$ months.

\subsection{Anxiety behavior in Tg4-42 mice}

The elevated plus maze test was used to assess anxiety-like behavior in Tg4-42 mice (Campos et al., 2013). The time spent in the open arms was used as an indication of open space-induced anxiety. Anxiety reduction is indicated by an increased stay in the open arms (Komada et al., 2008). The anxiety levels of 3-, 8- and 12-month-old Tg4-42 as well as 3- and 8-month-old Tg4-42 hom were examined. Transgenic mice showed no altered anxiety phenotype compared to same-aged wild-type mice (FIGURE $3.8 \mathrm{~A}$, two-way repeated measures ANOVA, genotype: $p=0,169783)$. In addition, the distance travelled did not differ between Tg4-42, Tg4-42 hom and same-aged WT mice (FIGURE 3.8 B, twoway repeated measures ANOVA, genotype: $p=0,138556)$. All mice showed an equal age-dependant decreased anxiety behavior (two-way repeated measures ANOVA, age: $p$ 
$=0,001841$ ) and decreased agitation with age (two-way repeated measures ANOVA, age: $p=0,012224)$.
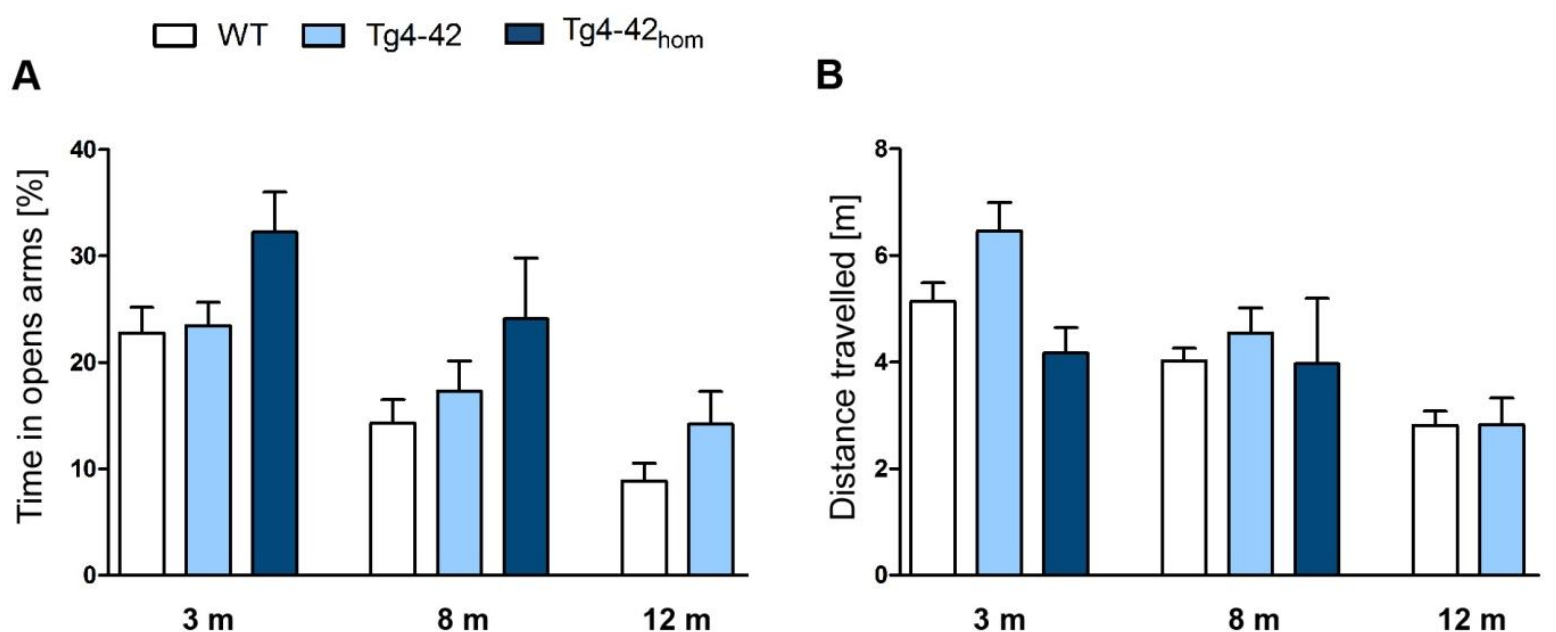

FIGURE 3.8 Unaltered anxiety behavior in Tg4-42 mice. (A) There was no significant difference between Tg4-42 and WT in the time spent in open arms and the (B) distance travelled at 3, 8 and 12 months of age. Furthermore, $\mathrm{Tg} 4-42_{\text {hom }}$ performed not significantly different to WT mice at 3 and 8 months in (A) the percentage time spent in open arms and (B) the distance travelled. Twoway repeated measures ANOVA. $n=10-15$ per group; data presented as mean \pm S.E.M; $m=$ month.

\subsection{Working memory in Tg4-42 mice}

Working memory in Tg4-42 mice was assessed in the cross maze by examining the alternation behavior of mice. Healthy mice tend to alternate between the arms of the maze and typically prefer to explore the least recently visited arm of the maze rather than returning to the one previously visited (Wietrzych et al., 2005). Working memory of 3-, 8and 12-month-old Tg4-42 as well as 3- and 8-month-old Tg4-42 hom was investigated in the cross maze. No significant difference was detected between transgenic and wild-type animals in spontaneous alternation independent of age (FIGURE 3.9 A two-way repeated measures ANOVA, genotype: $p=0,784478$, age: $p=1,000001)$. In addition, Tg4-42 and Tg4-42 hom travelled an equivalent distance as same-aged wild-type mice at all ages examined (FIGURE 3.9 B, two-way repeated measures ANOVA, genotype: $p=0,445393$, age: $p=0,796430)$. 


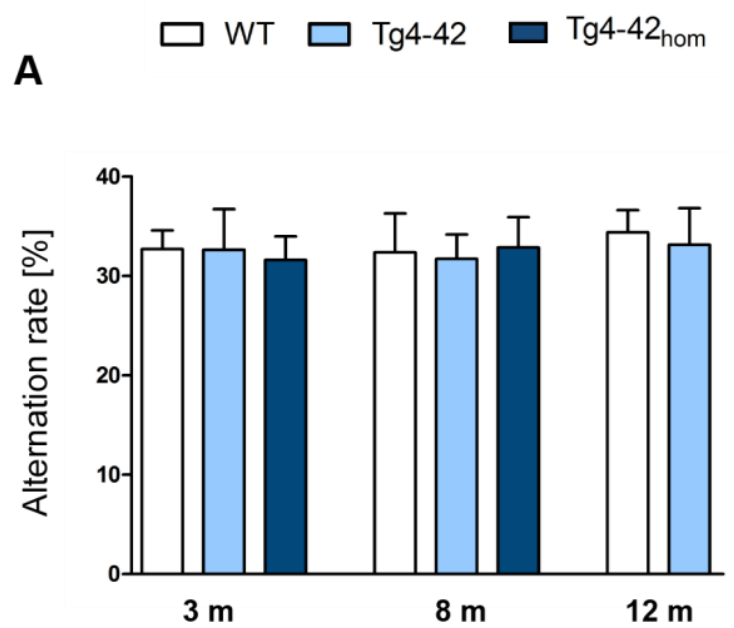

B

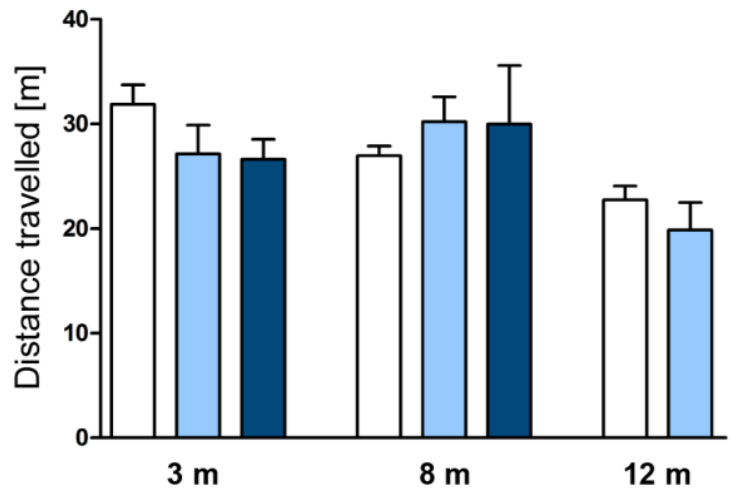

FIGURE 3.9 Intact working memory in Tg4-42 mice. (A) Alternation rate and (B) distance travelled did not alter between 3, 8 and 12 months old Tg4-42 and same-aged WT mice. Furthermore, no differences in alternation rate or distance travelled was detected between 3 and 8 months Tg4-42 hom and WT mice. Two-way repeated measures ANOVA. $n=9-15$ per group, data presented as mean \pm S.E.M; $m=$ month.

\subsubsection{5}

\section{Spatial memory deficits in Tg4-42 mice}

Spatial reference memory in Tg4-42, $\mathrm{Tg} 4-42_{\text {hom }}$ and wild-type mice was assessed using the Morris water maze. Hemizygous Tg4-42 mice and wild-type littermates were tested at three, eight and twelve months of age. Homozygous Tg4-42 (Tg4-42 hom) mice were examined at three and eight months of age.

Testing began with three days of cued training, were mice familiarized with the pool and learned to locate a marked platform. All mice reached criteria by showing progressively decreased escape latencies or reaching the platform in under 15 seconds after three days of training (FIGURE 3.10 A-C; two-way repeated measures ANOVA, $3 \mathrm{~m}$ and $8 \mathrm{~m}$ : main effect of days: $p<0,000001 ; 12 \mathrm{~m}$ : main effect of days: $p<0,000226)$. Swimming speed did not differ between genotypes at any tested time point (FIGURE 3.10 A-C; two-way repeated measures ANOVA, genotype: $p=0,271846)$. The cued training revealed that all mice are able to perform the water maze task as they have an intact vision and the motor abilities to swim.

Twenty-four hours after the cued training, mice performed five days of acquisition training (FIGURE 3.11). Their learning abilities to find the location of a submerged platform by using proximal and distal cues was tested. For the escape latency a significant main effect of age could be found (two-way repeated measures ANOVA, age: $p=$ 0,036700). In contrast, swimming speed did not significantly alter between three, eight 
and twelve month old animals (FIGURE 3.11 A -C; two-way repeated measures ANOVA, age: $p=0,776269$ ). These results show that younger mice performed superior to older animals while this difference was not due to differences in swimming speed due to agerelated motor deficits. Furthermore, the acquisition phase revealed a significant main effect of the factor genotype for the escape latencies (two-way repeated measures ANOVA, genotype: $p=0.012561$ ), while the swimming speed was not significantly affected by the genotype of animals (two-way repeated measures ANOVA, genotype: $p=$ 0.214047). Across the five days of acquisition the animals, irrespective of genotype and age, showed a significant decrease in the escape latencies. At three months of age, Tg4-42, Tg4-42 hom and WT mice improved significantly over the five days and showed no differences between the genotypes (FIGURE $3.11 \mathrm{~A}$; two-way repeated measures ANOVA, days: $p=0,000001$; genotype: $p=0.106729$ ). At eight and twelve months of age, the escape latencies decreased significantly over the training days (FIGURE 3.11 B and $\mathrm{C}$, two-way repeated measures ANOVA, $8 \mathrm{~m}$ days: $p=0,000001 ; 12 \mathrm{~m}$ days: $p=$ $0,000002)$. However, a significant difference between the groups was detected in both age groups (two-way repeated measures ANOVA, $8 \mathrm{~m}$ genotype: $p=0,004182 ; 12 \mathrm{~m}$ genotype: $p=0,010909$ ). Eight months old Tg4-42 hom performed significantly inferior to WT mice on day one, two, four and five of the acquisition training (FIGURE 3.11 B; t-test: day 1: $p=0,011037$; day $2: p=0,012627$; day $4: p=0,007121$; day $5: p=0,007482$ ). Furthermore, twelve months old Tg4-42 showed significantly longer escape latencies on day four and five (FIGURE $3.11 \mathrm{C}$; t-test: day 4: $p=0,003086$; day $5: p=0,023866$ ). These results suggest a slightly impaired spatial learning in twelve months old Tg4-42 and eight months old $\mathrm{Tg} 4-42_{\text {hom }}$ mice in the Morris water maze.

Twenty-four hours after the last acquisition trial, a probe trial was conducted to assess spatial reference memory. At three months of age, WT, Tg4-42 and Tg4-42 mice displayed a significant preference for the target quadrant, as indicated by the percentage time spent in the four quadrants of the pool (FIGURE $3.12 \mathrm{~A}$, one-way ANOVA, WT: $p<0.001$ target vs. all other quadrants; Tg4-42: $p<0.001$ target vs. left quadrant, $p<0.01$ target vs. right and opposite quadrant; Tg4-42 hom: $p<0.001$ target vs. all other quadrants). Eight months old Tg4-42 and WT mice also spent a significant higher percentage of time in the target quadrant in comparison to the other quadrants. However, eight months old Tg4-42 hom mice displayed no significant preference for the target quadrant indicating spatial reference memory deficits (FIGURE 3.12 B, one-way ANOVA, WT: $p<0.001$ target vs. all other quadrants; Tg4-42: $p<0.001$ target vs. all other quadrants). Furthermore, twelve months old Tg4-42 mice showed no quadrant preference, while same-aged WT mice still demonstrated a significant preference for the target quadrant (FIGURE 3.12 C, one-way ANOVA, WT: $p<0.001$ target vs. all other 
quadrants). Aged Tg4-42 and Tg4-42 hom showed an impaired spatial learning reflected by the absence of a preference for the target quadrant during the probe trial. Swimming speed did not differ during the probe trial between the groups at any age tested (FIGURE 3.12 A - C).

In summary, the results of the acquisition training and the probe trial suggest that $A \beta_{4-42}$ expression in the transgenic mice impairs spatial learning and spatial reference memory in the Morris water maze.

A
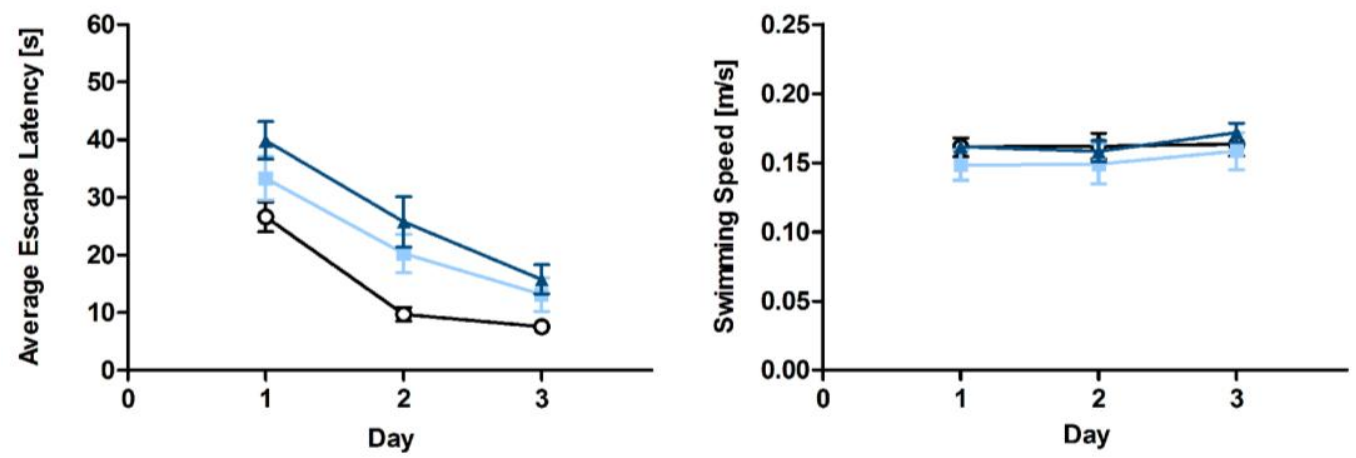

B
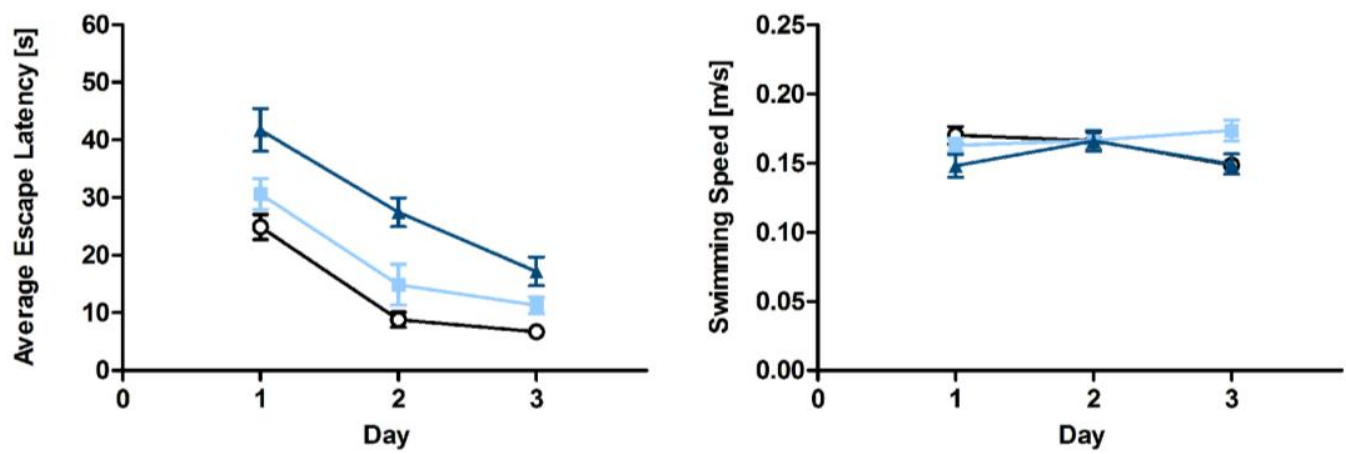

C
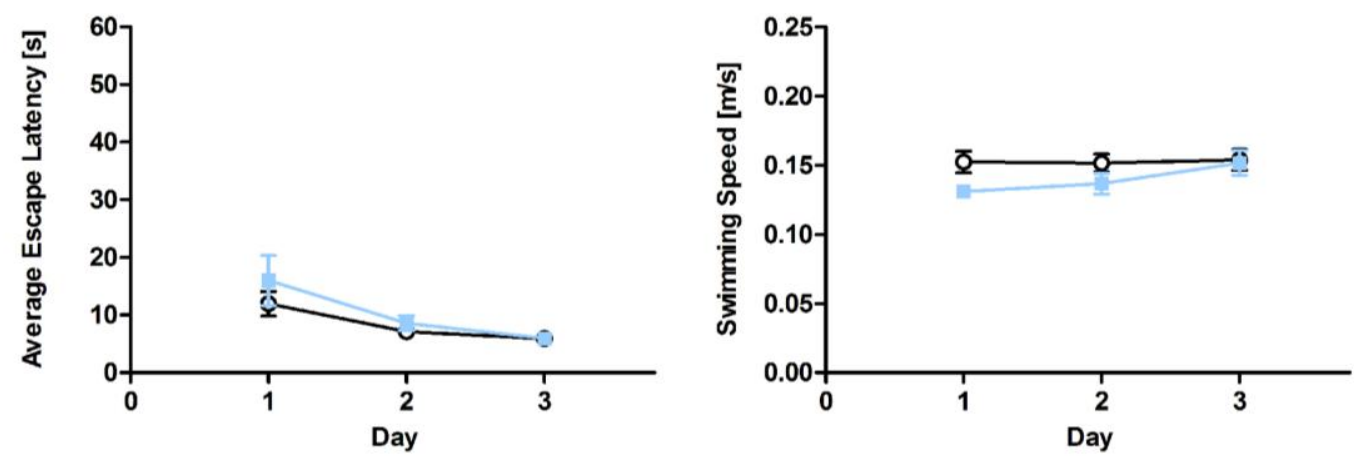

$8 \mathrm{~m}$

$12 \mathrm{~m}$

FIGURE 3.10 Cued training of the water maze reveals that Tg4-42 exhibit intact eyesight and the motor abilities to perform the test. Hemizygous Tg4-42 mice and WT littermate controls were examined at (A) 3, (B) 8 and (C) 12 months of age. In addition, Tg4-42 hom were tested at (A) 3 and (B) 8 months of age. All mice showed progressively shorter escape latencies in response to training and reached the criteria that allows continuation of the water maze. Swimming speed did not differ between genotypes at any tested time point (A)-(C). Escape latencies and swimming speed: two-way repeated measures ANOVA. $n=12-15$ per group; data presented as mean \pm S.E.M; $\mathrm{m}=$ months. 
A

- WT $-\mathrm{Tg} 4-42 \neq \mathrm{Tg} 4-42_{\text {hom }}$
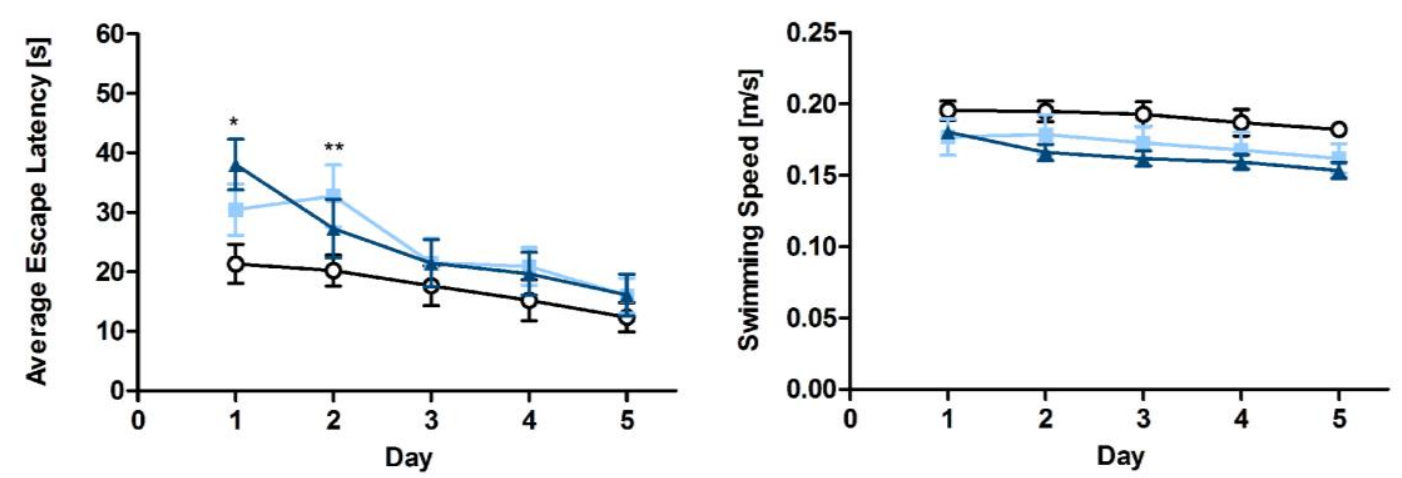

$3 \mathrm{~m}$

B
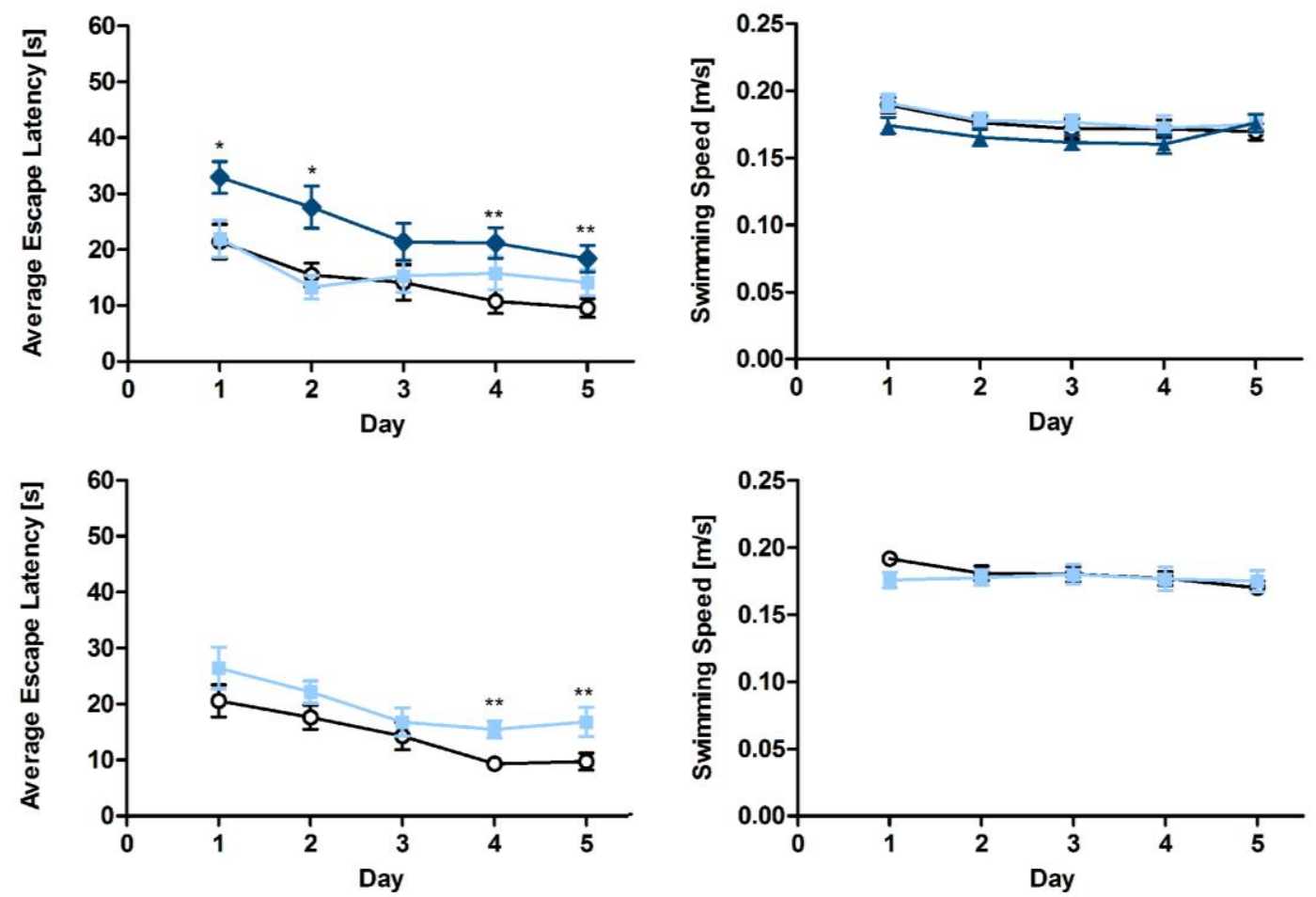

$12 \mathrm{~m}$

FIGURE 3.11 Spatial learning was assessed in the acquisition training of the Morris water maze. Hemizygous Tg4-42 mice and WT littermate controls were examined at (A) 3, (B) 8 and (C) 12 months of age. In addition, Tg4-42hom were tested at (A) 3 and (B) 8 months. Across the 5 days of acquisition all groups showed a significant decrease in the escape latencies. (A) Three months old Tg4-42 and Tg4-42 hom displayed similar learning curves during the acquisition training. (B) At 8 months Tg4-42 hom performed significantly inferior to WT and Tg4-42 . Furthermore, (C) 12-monthold WT performed significantly better than same-aged Tg4-42 mice. Swimming speed did not differ between genotypes at any tested time point (A)-(C). Escape latencies and swimming speed: two-way repeated measures ANOVA, ${ }^{* * *} \mathrm{p}<0.001,{ }^{* *} \mathrm{p}<0.01,{ }^{*} \mathrm{p}<0.05 . \mathrm{n}=12-15$ per group; data presented as mean \pm S.E.M; $m=$ months. 
A
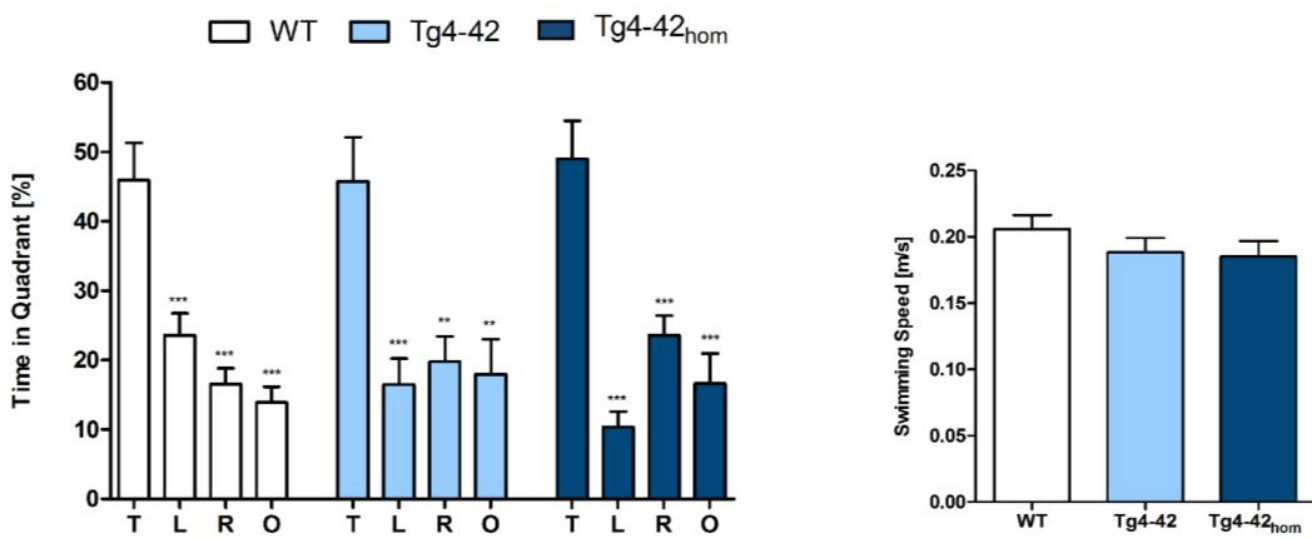

$3 \mathrm{~m}$

B
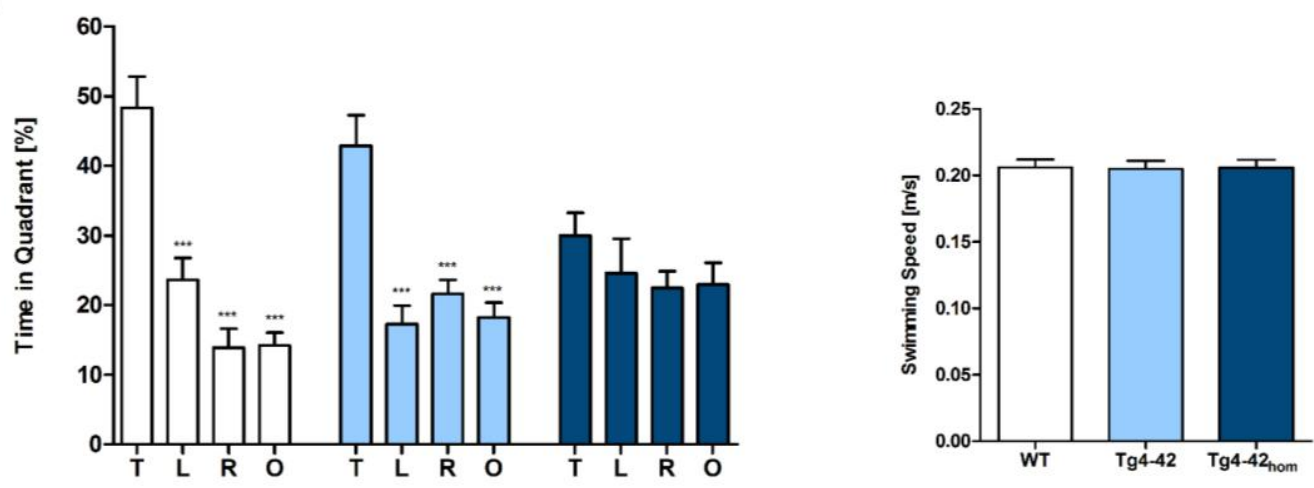

$8 \mathrm{~m}$

C
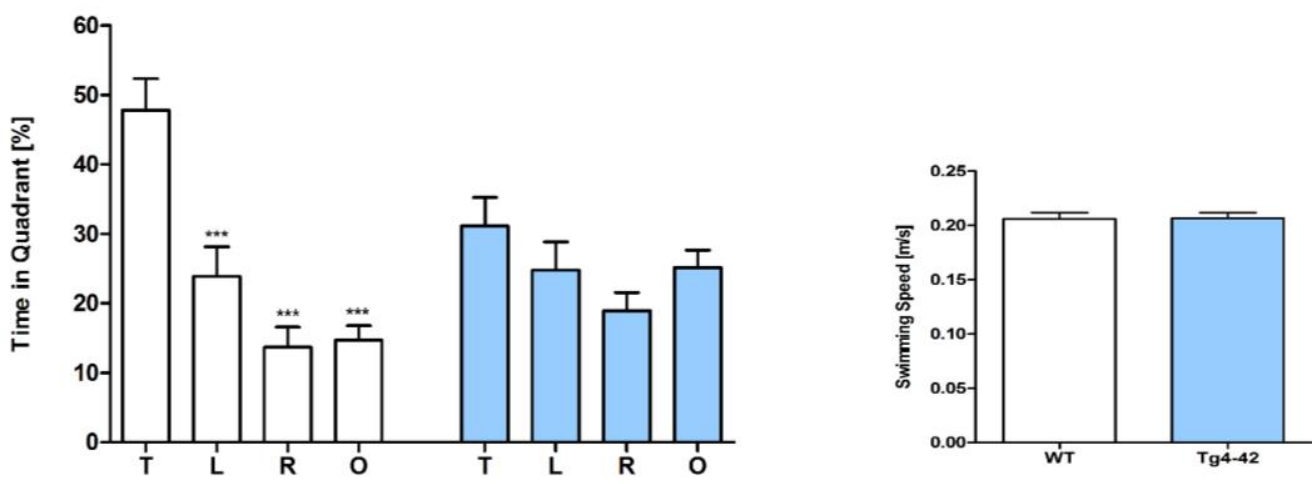

$12 \mathrm{~m}$

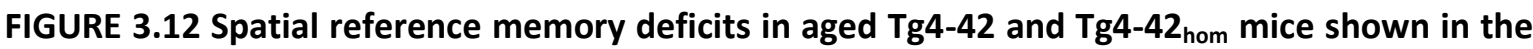
probe trial of the Morris water maze. Hemizygous Tg4-42 mice and WT littermate controls were tested at (A) 3, (B) 8 and (C) 12 months of age. In addition, Tg4-42 hom were examined at (A) 3 and (B) 8 months of age. (A) At 3 months of age all groups spent a greater percentage of time in the target quadrant (WT: $p<0.001$ T vs. all other quadrants; Tg4-42: $p<0.001$ T vs. $L, p<0.01$ T vs. R and 0; Tg4-42 hom: $\mathrm{p}<0.001 \mathrm{~T}$ vs. all other quadrants). (B) Probe trial revealed a significant reduced learning behavior for 8-month-old Tg4-42 hom mice as they showed no preference for the target quadrant. WT and Tg4-42 mice showed no learning deficits at this age (WT and Tg4-42: $p<0.001$ T vs. all other quadrants). (C) At 12 month of age, hemizygous Tg4-42 mice did not show a preference for the target quadrant. However, same-aged WT mice still had a significant preference for the target quadrant (WT and Tg4-42: $p<0.001 \mathrm{~T}$ vs. all other quadrants). (A-C) Swimming speed did not differ between the groups. Abbreviations: $T$ = target quadrant; $L=l e f t$ quadrant; $\mathrm{R}$ = right quadrant; $\mathrm{O}=$ opposite quadrant. Quadrant preference and swimming speed: One-way analysis of variance (ANOVA) followed by Bonferroni multiple comparisons, $* * * p<$ $0.001 ; * * \mathrm{p}<0.01$. $\mathrm{n}=12-15$ per group; data presented as mean \pm S.E.M; $\mathrm{m}=$ months. 


\subsection{Decreased contextual learning in Tg4-42 mice}

Contextual and tone fear conditioning were tested in 12-month-old Tg4-42 and WT mice. During the training session involving a tone-foot-shock pairing (CS/US) both genotypes exhibited comparable degrees of freezing (FIGURE 3.13 A).

A

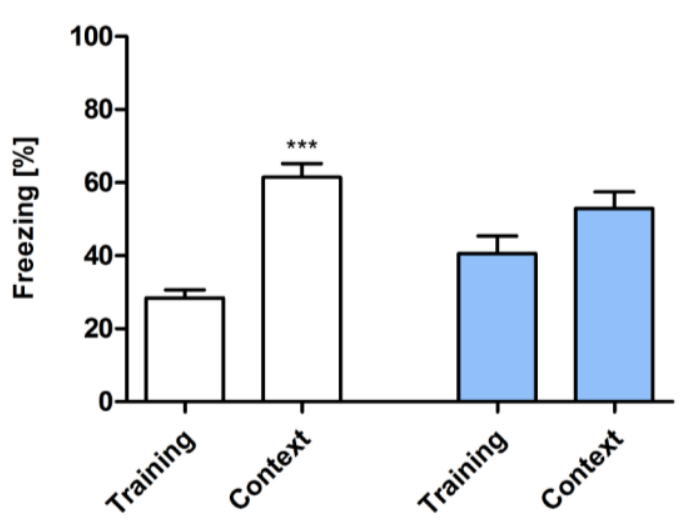

B

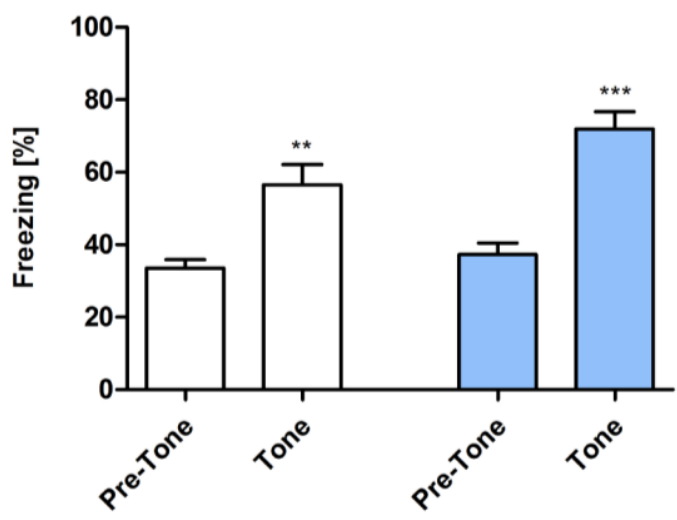

C
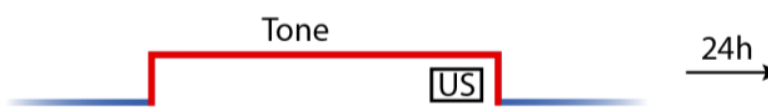

Re-exposure to context (CS)

D
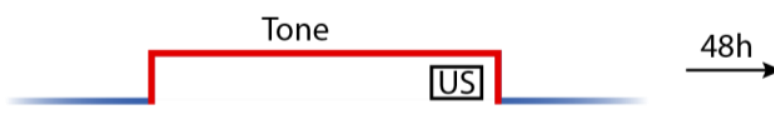

Tone (CS)

FIGURE 3.13 Impaired contextual conditioning in Tg4-42 mice. 12-month-old Tg4-42 and WT mice were trained with a CS/US pairing for contextual fear conditioning. (A, C) Mice were reintroduced to the original training context (CS) 24 hours post training and tested for contextual memory. Levels of freezing during the re-exposure were not significantly different from the training trial for Tg4-42 mice. In contrast, WT mice showed a significant increased freezing response to the context. (B, D) 48 hours post training mice were introduced to an altered chamber and tested for tone memory. Tg4-42 and WT showed an increased freezing response due to the tone (CS). (C, D): the blue line indicates the chamber, the red line the tone and the green line the altered chamber. $\mathrm{CS}=$ conditioned stimulus. US $=$ unconditioned stimulus. Freezing: paired t-test, ${ }^{* * *} \mathrm{p}<0.001,{ }^{* *} \mathrm{p}<0.01 . \mathrm{n}=18-20$ per group; .

When mice were tested 24 hours after the training trial for context fear conditioning (FIGURE 3.13 C), Tg4-42 mice demonstrated no significantly increased freezing behavior in response to the conditioning chamber. In contrast, WT mice displayed significantly increased freezing (FIGURE $3.13 \mathrm{~A}$, paired t-test, $p<0.001$ ) as a response to the context. 
Tg4-42 mice jumped and vocalized in response to the electric foot-shock to a similar degree as WT mice, suggesting normal pain perception in these mutant mice. However, transgenic mice were not able to attribute the pain of the foot-shock during the training trial to the context. Therefore, Tg4-42 showed an impaired contextual learning.

Twenty-four hours after the context testing (48 hours after training), the same mice were tested for conditioned fear of the tone (FIGURE 3.13 D). Therefore mice were reintroduced to the altered fear conditioning chamber. When the tone was presented without the foot-shock, both Tg4-42 and WT mice exhibited similar freezing responses (FIGURE $3.13 \mathrm{~B}$ ). In both mouse lines freezing increased significantly compared to the pre-tone period (paired t-test, WT $p<0.01$; Tg4-42: $p<0.001$ ). Therefore, all mice associated the tone with the previously received foot-shock and showed no impaired tone learning.

\subsubsection{Long-term exposure to $\mathrm{N}$-truncated $A \boldsymbol{\beta}_{4-42}$ induces neuron loss}

In order to investigate the effects of long-term exposure to $\mathrm{N}$-truncated $A \beta_{4-42}$ in $\mathrm{Tg} 4-42$ and $\mathrm{Tg} 4-42_{\text {hom }}$ mice the neuron number in the CA1 layer of the hippocampus was examined via DAPI staining and unbiased stereology.

DAPI staining revealed an obvious neuron loss in the CA1 layer of the hippocampus between eight months old wild-type mice and same-aged Tg4-42 mice (FIGURE 3.14 B). An even more pronounced neuron loss was observed in eight months old Tg4-42 hom mice (FIGURE 3.14 C).

In order to quantify the neuron numbers in the CA1 in an unbiased way designbased stereology was performed. The hippocampal pyramidal cell layer CA1 was quantified from Bregma -1.22 to -3.80 . Stereology revealed an age- and genotypedependent neuron loss in Tg4-42 and Tg4-42 hom mice (FIGURE 3.14 D, one-way repeated measures ANOVA, genotype: $p=0,000094$; age: $p=0,000011)$. At three months of age no significant difference in the number of neurons could be detected between wild-type $($ mean $=304,805$, SEM $\pm 12,560), \operatorname{Tg} 4-42$ (mean $=309,600, \operatorname{SEM} \pm 17,570)$ and Tg4-42 hom mice. However, Tg4-42 hom (mean = 244,808, SEM $\pm 21,320$ ) exhibited fewer neurons in the CA1 than same-aged Tg4-42 and WT mice. At eight months stereology revealed a $38 \%$ neuron loss in hemizygous Tg4-42 mice (mean $=178.014$, SEM $\pm 9,083$, one-way ANONA: $p<0.01$ ) compared to wild-type littermate controls (mean $=282.683$, SEM $\pm 15,670)$. In same-aged homozygous Tg4-42 hom mice the neuron loss was even more pronounced with a $65 \%$ decline (mean $=97,600$, SEM $\pm 14,300$, one-way ANONA: $p<0.001$ ) to WT controls. Homozygous 8-month-old Tg4-42 mice displayed $46 \%$ fewer cells than same-aged hemizygous Tg4-42 mice (one-way ANONA: $p<0.01$ ). 
A

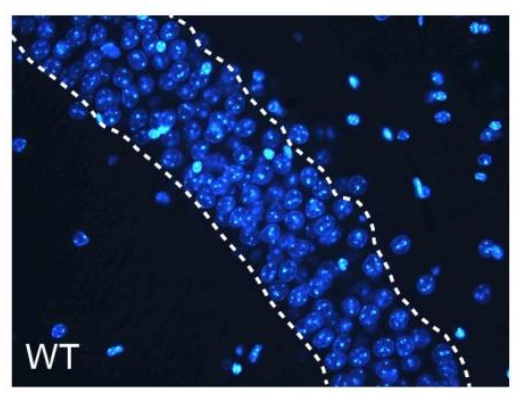

B

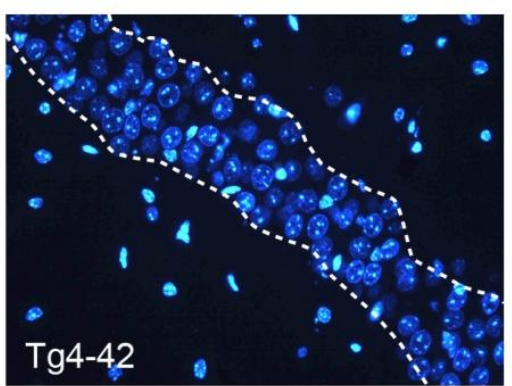

C

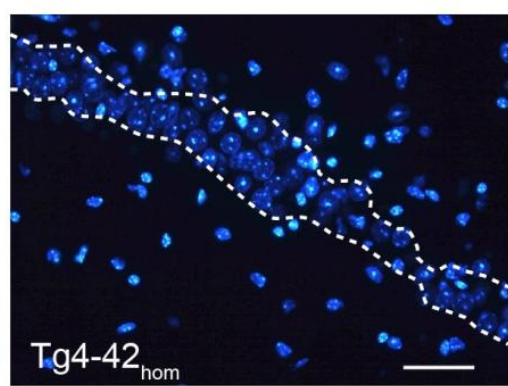

D

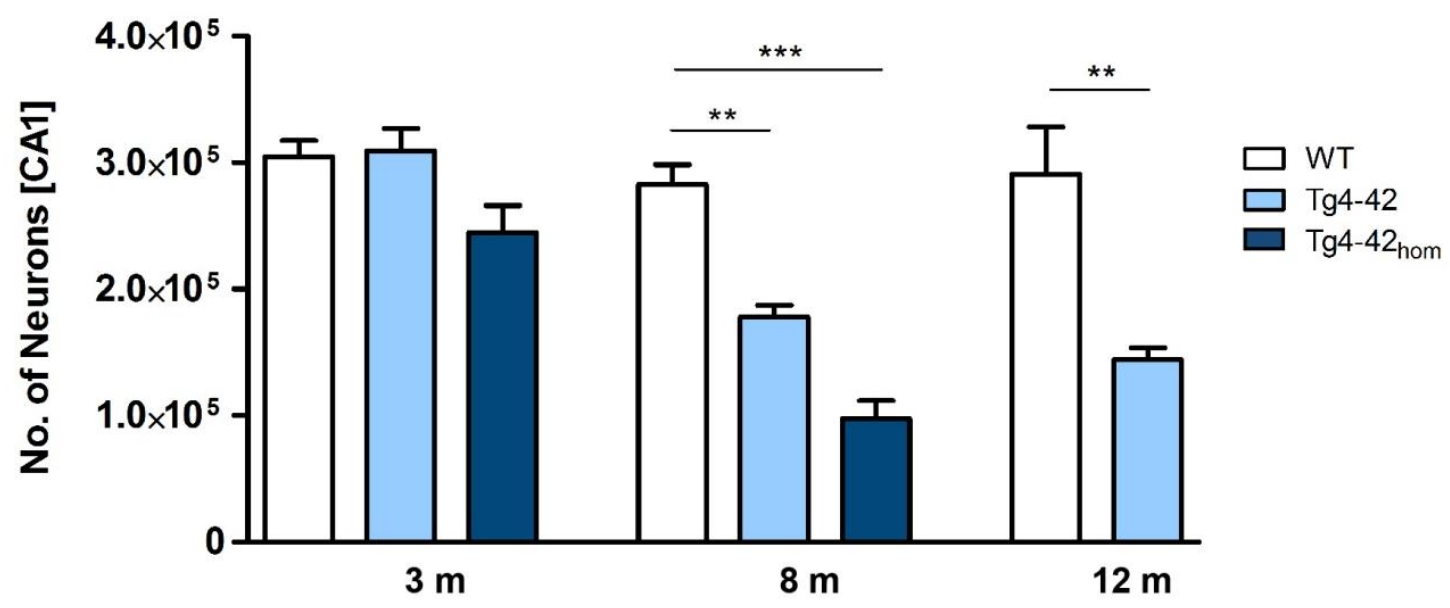

$\mathbf{E}$

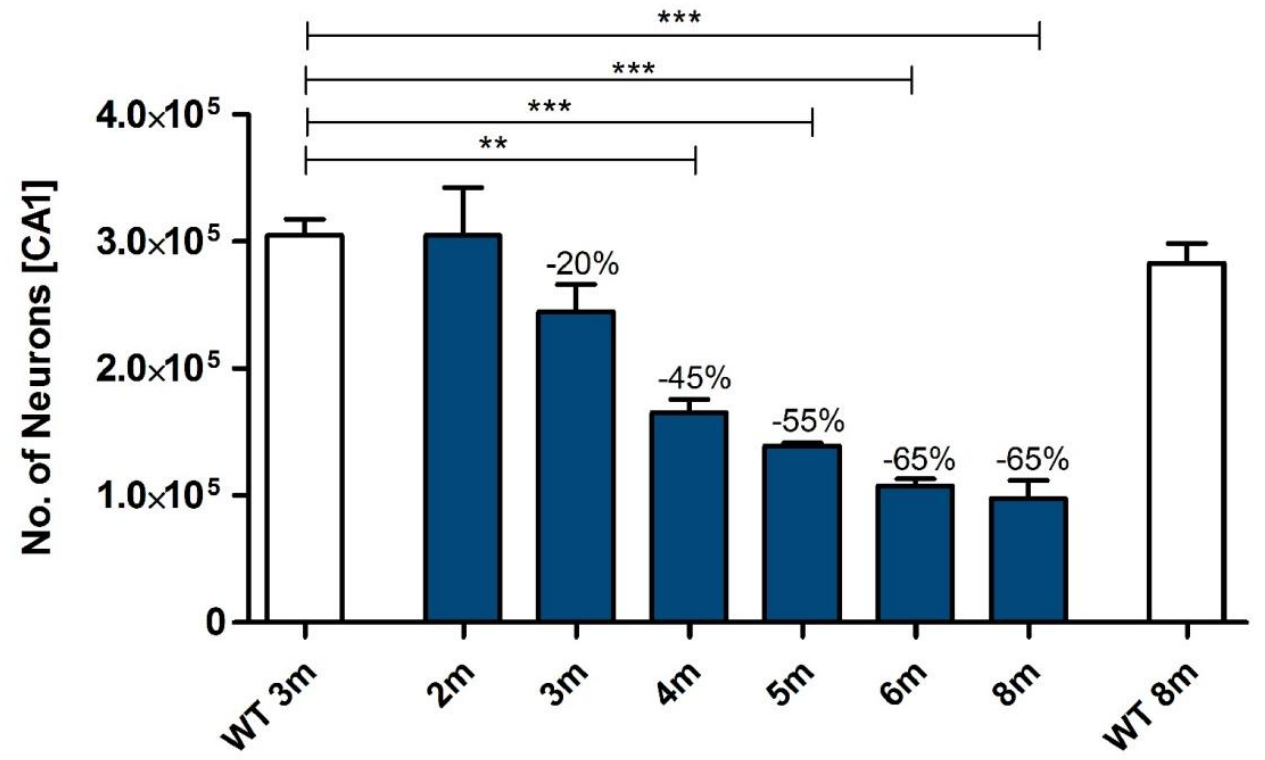

FIGURE 3.14 Age- and dose-dependent neuron loss in hippocampus of Tg4-42 mice. (A-C) DAPI staining revealed obvious neuron loss in the CA1 layer of the hippocampus in (B) eight months old Tg4-42 compared to same-aged (A) WT mice. (C) Neuron loss in eight months old Tg4-42 hom was even more pronounced. (D-E) Quantification of neurons in CA1 using unbiased stereology. Oneway ANOVA; $\mathrm{n}=3-5 ;{ }^{* *} p<0.01,{ }^{* * *} p<0.001$; data presented as mean \pm S.E.M; $\mathrm{m}=$ months. Scale bar: (A-C) $50 \mu \mathrm{m}$. 
Furthermore, stereology revealed a $51 \%$ loss in neuron number in the CA1 at the age of twelve months between hemizygous Tg4-42 (mean $=144,057, \mathrm{SEM} \pm 9,540$, one-way ANONA: $p<0.01$ ) and same-aged WT mice (mean $=291,053, \mathrm{SEM} \pm 37,530)$. Between eight and twelve months, the neuron loss in hemizygous mice increased by $20 \%$. In contrast, wild-type mice showed no age-dependant neuron loss.

In order to further investigate the neuron loss in Tg4-42 hom mice the number of neurons was additionally counted at two, four, five and six months of age (FIGURE 3.14 E). The number of neurons in 2-month-old Tg4-42 $2_{\text {hom }}$ (mean $=304,705$, SEM $\pm 37,750$ ) was comparable to 3-month-old old WT mice and not significantly different from 3-monthold Tg4-42 hom. However, 4-, 5- and 6-month-old homozygous mice displayed a significant neuron loss of $45 \%, 55 \%$ and $65 \%$, respectively (one-way ANONA: $4 m$ : mean = 165,244, SEM $\pm 10,660, p<0.01 ; 5 m$ : mean $=139,169$, SEM $\pm 2,218, p<0.001 ; 6 m$ : mean $=107,377$, SEM $\pm 5,760, p<0.001$ ). The neuron number in homozygous mice followed a exponential decay that seems to led to a plateau starting at six months with a neuron loss of $65 \%$.

In summary, it can be stated that long-term exposure to $\mathrm{N}$-truncated $A \beta_{4-42}$ induces an age- and dose-dependent neuron loss hemizygous and homozygous Tg4-42 in the CA1 layer of the hippocampus. 

decline and neuron loss in Tg4-42 and 5XFAD mice by deep sequencing

In the second part of the thesis, a comparative gene expression analysis of $\mathrm{Tg} 4-42$ and 5XFAD brain tissue was performed using next-generation sequencing. The wellestablished plaque developing 5XFAD mouse model (Oakley et al., 2006; Jawhar et al., 2010) was compared with the Tg4-42 mouse model that solely expresses $A \beta_{4-42}$ without extracellular plaque depositions (see section 3.1.3.2). Furthermore, memory deficits in these mouse models were analyzed and compared. In this part of the study only female mice were used. Young (3- to 4-month-old) and aged Tg4-42 (12-month-old) mice were compared with same-aged 5XFAD and wild-type mice. The aim of this study was to compare the behavior deficits and to elucidate the similarities and distinctions in expression profiles of these two AD mouse models.

\subsubsection{Amyloid beta accumulation in Tg4-42 and 5XFAD mice}

Transgene expression in $\operatorname{Tg} 4-42$ and 5XFAD mice was analyzed using immunohistochemistry.

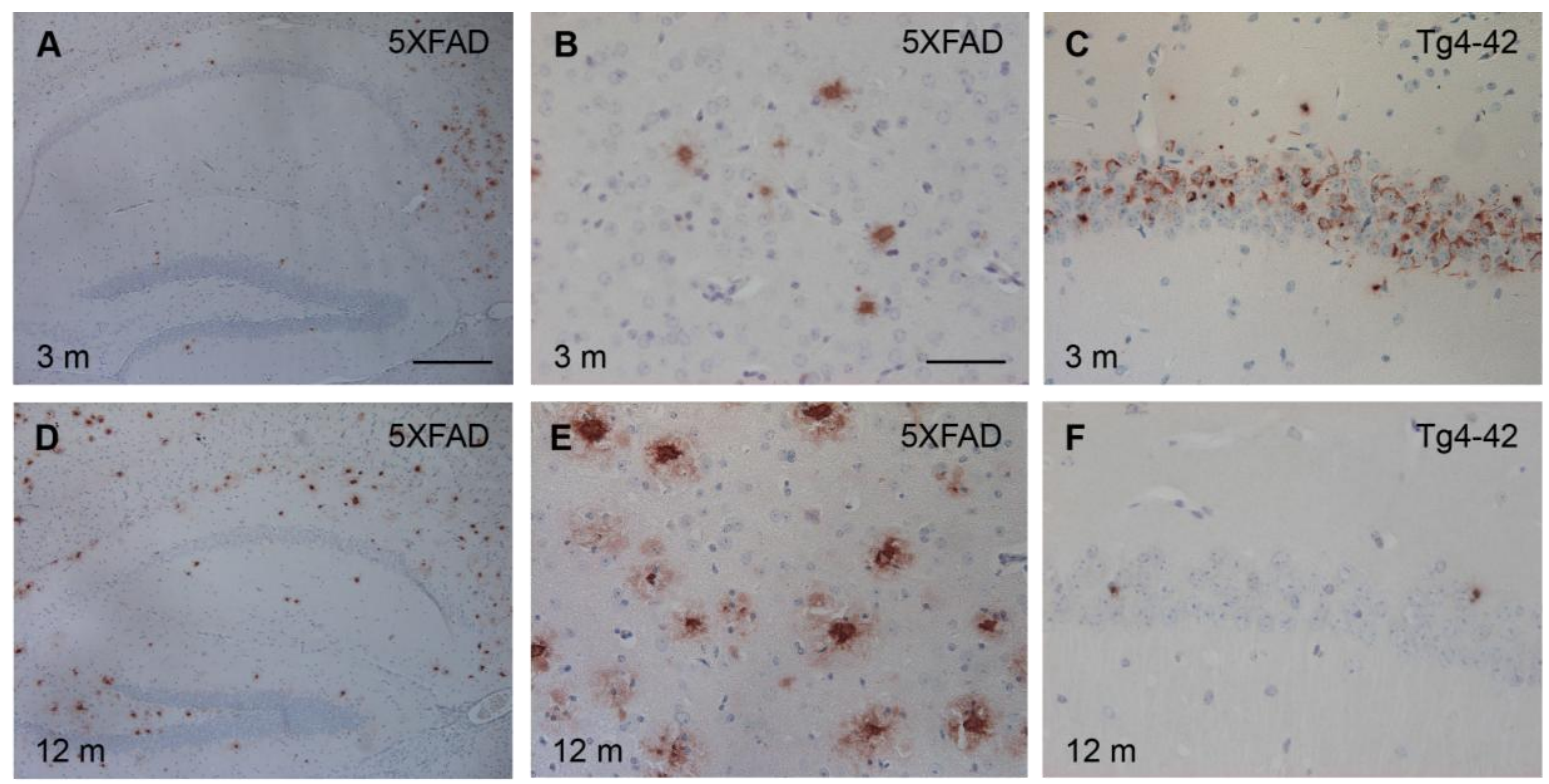

FIGURE 3.15 Immunohistochemical staining of 5XFAD and Tg4-42 mice. (A-B) Immunostaining demonstrating $A \beta$ accumulation in cortex of 3 months old 5XFAD. (D-E) 12 months old 5XFAD mice exhibited more intensive cortical plaque staining. (C) Abundant intraneuronally $A \beta$ staining in the CA1 of the hippocampus in 3 months old Tg4-42 mice. (F) A $\beta$ immunostaining declined in 12 months old Tg-42 mice. Antibody: (A-B) polyclonal antiserum 24311. Abbreviations: $m=$ months. Scale bar: (A and D) $200 \mu \mathrm{m}$; (B-C, E-F) $50 \mu \mathrm{m}$.

Immunohistochemical detection of $A \beta$, using the pan- $A \beta$ antibody $2431-1$, showed an abundant plaque-associated pathology in 3- and 12-month-old 5XFAD mice. Plaque 
pathology increased with age (FIGURE 3.15 A-B vs. D-E). In contrast to 5XFAD mice, young Tg4-42 mice showed strong intracellular immunoreactivity predominantly in the CA1 region of the hippocampus (FIGURE 3.15 C). The CA1 A $\beta$ expression declined during aging (FIGURE 3.15 F; section 3.1.3.2).

\subsubsection{Tg4-42 and 5XFAD mice display spatial memory deficits}

Spatial reference memory was assessed in three and twelve months old female Tg4-42, 5XFAD and WT mice using the Morris water maze.

To rule out effects from possible motor or sensory deficits and to familiarize with the pool mice performed three days of cued training with a marked platform. WT, Tg4-42 and 5XFAD mice showed progressively decreased escape latencies at all ages tested and reached the goal platform after three consecutive training days in under $15 \mathrm{sec}$ and therefore reached criteria (FIGURE 3.16). Furthermore, no overall differences in swimming speed could be detected between the genotypes (FIGURE 3.16, two-way repeated measures ANOVA, genotype: $p=0,1564)$. The cued training revealed that all mice had an intact vision and appropriate motor abilities to swim.

Twenty-four hours after the cued training, mice were subjected to acquisition training in order to test their learning abilities to find the location of a submerged platform using distal and proximal cues. A significant main effect of genotype for escape latencies (two-way rrepeated measures ANOVA, genotype: $p=0.0414$ ) was found. Young Tg4-42, 5XFAD and WT mice showed a significant decrease in the escape latencies to reach the hidden platform (FIGURE 3.17 A, two-way repeated measures ANOVA, escape latency: $p$ $=0.000011)$. Moreover, aged WT animals showed a significant decrease in the escape latencies while the escape latencies for aged Tg4-42 and 5XFAD did not improve over the five days of training (FIGURE 3.17 B, two-way repeated measures ANOVA, effect of genotype for escape latency: $p=0.0001)$. Aged 5XFAD mice performed significantly inferior to WT mice on day two, three, four and five of the acquisition training (t-test, escape latencies WT vs. 5XFAD: 2 day: $p=0,034007,3$ day: $p=0,037330,4$ day: $p=$ 0,003085, 5 day: $p=0,001532$ ). Furthermore, aged Tg4-42 mice showed significantly longer escape latencies on day four and five (t-test, escape latencies WT vs. Tg4-42: 4 day: $p=0,022892,5$ day: $p=0,007812)$. In contrast, the swimming speed across the five days of acquisition training showed no significant differences irrespective of genotype and age (FIGURE 3.17 A-B, two-way repeated measures ANOVA, $p=0.0566$ ). Young Tg4-42 and 5XFAD animals performed superior to older animals while this difference was not due to differences in swimming velocity due to age-related motor deficits. These results suggest that spatial learning is impaired in aged female Tg4-42 and 5XFAD mice. 
Twenty-four hours after the last acquisition trial, a probe trial was performed to assess spatial reference memory. Young Tg4-42, 5XFAD and WT mice displayed a significant preference for the target quadrant, as indicated by the percentage time spent in different quadrants of the pool (FIGURE 3.18, one-way ANOVA, WT: $p<0.001$ target vs. all other quadrants; 5XFAD: $p<0.001$ target vs. left and opposite quadrant, $p<0.01$ target vs. right quadrant; Tg4-42: $p<0.001$ target vs. opposite quadrant, $p<0.01$ target vs. right and left quadrant). No quadrant preference was found for aged Tg4-42 and 5XFAD mice, while WT mice still demonstrated significant preference for the target quadrant at that time point (FIGURE 3.18, one-way ANOVA, WT: $p<0.001$ target vs. left and opposite quadrant, $p<0.01$ target vs. right quadrant). Aged 5XFAD mice showed a reduced swimming speed during the probe trial (one-way ANOVA, 5XFAD vs WT: $p<$ $0,05 ; 5 X F A D$ vs Tg4-42: $p<0,01)$. The absence of a preference for the target quadrant as compared to the remaining quadrants during the probe trial demonstrates that aged Tg442 and 5XFAD mice display a robust deficit in spatial reference memory.

In summary, the results of the acquisition phase and the probe trial suggest that aged Tg4-42 and 5XFAD mice display an impaired spatial and spatial reference memory.

A
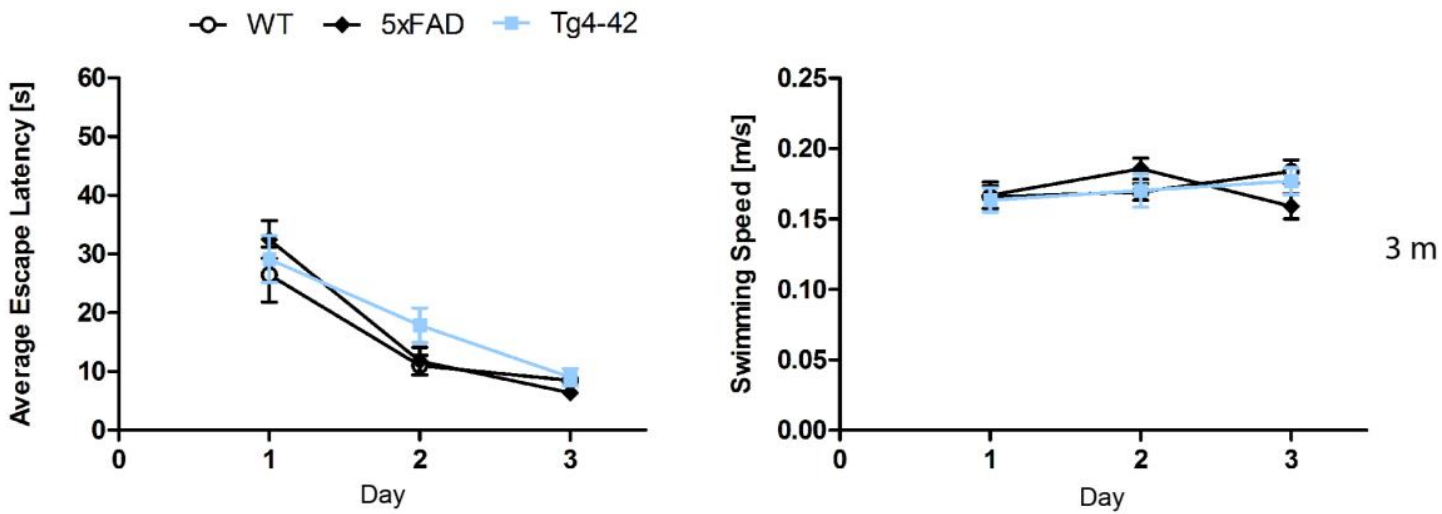

B
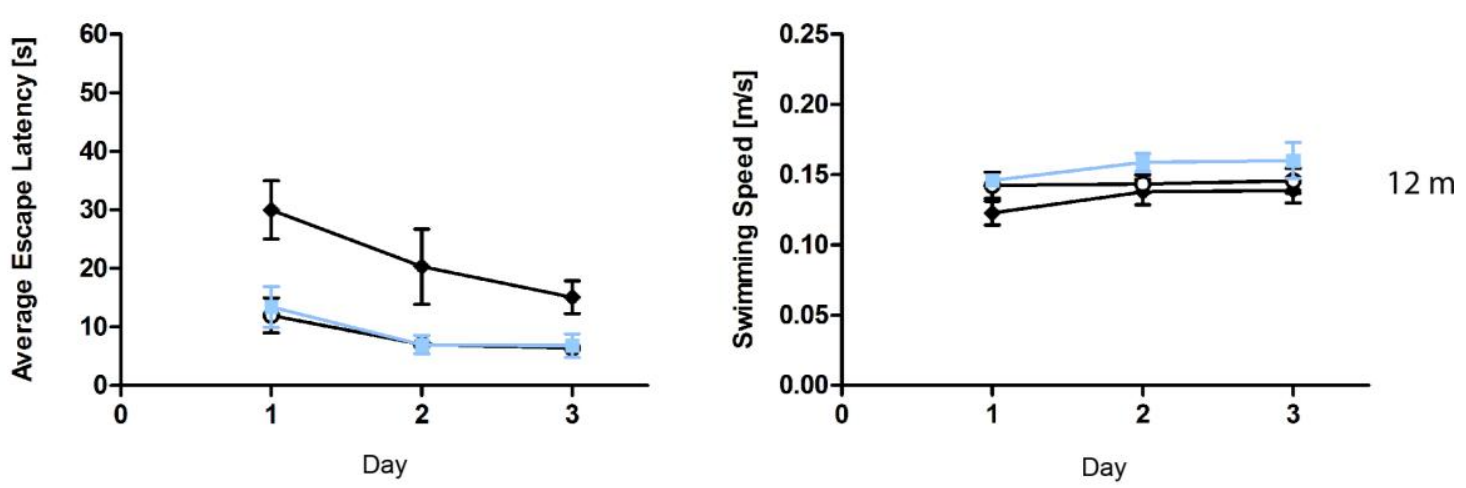

FIGURE 3.16 Cued training of the water maze reveals that Tg4-42 and 5XFAD exhibit intact eyesight and the motor abilities to perform the test. Female Tg4-42 and 5XFAD mice as well as WT littermate controls were examined at (A) 3 and (B) 12 months of age. All mice showed progressively shorter escape latencies in response to training and reached the criteria that allows continuation of the water maze. Swimming speed did not differ between genotypes at any tested time point (A-B). Escape latencies and swimming speed: two-way repeated measures ANOVA; $n=$ 8 - 11 per group; data presented as mean \pm S.E.M. 
A
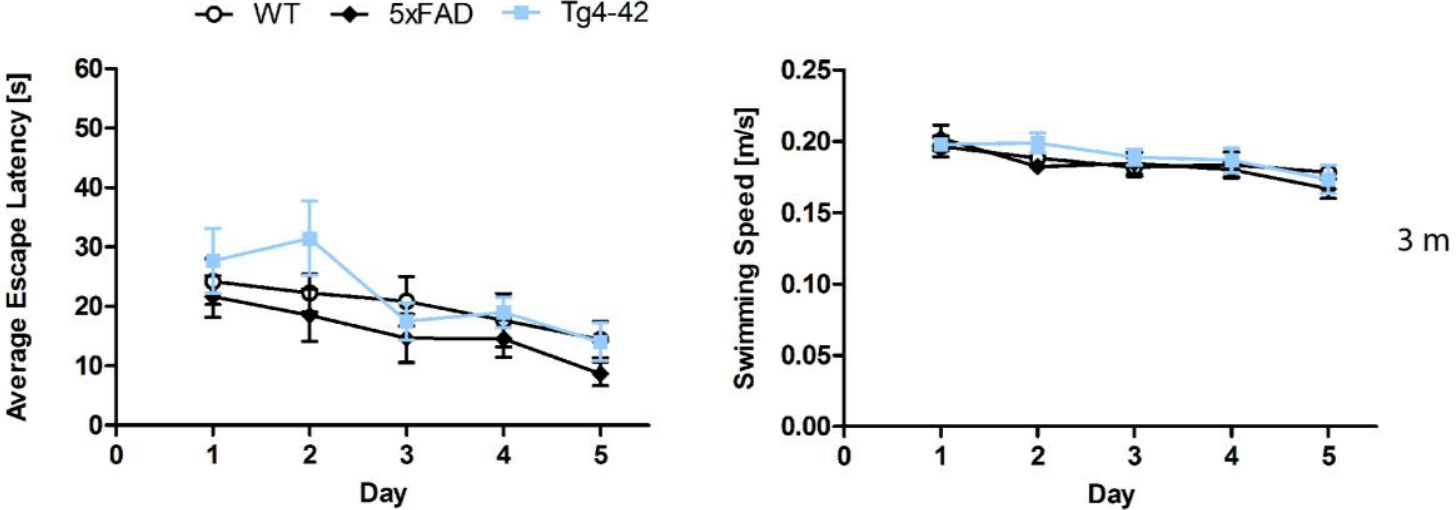

B
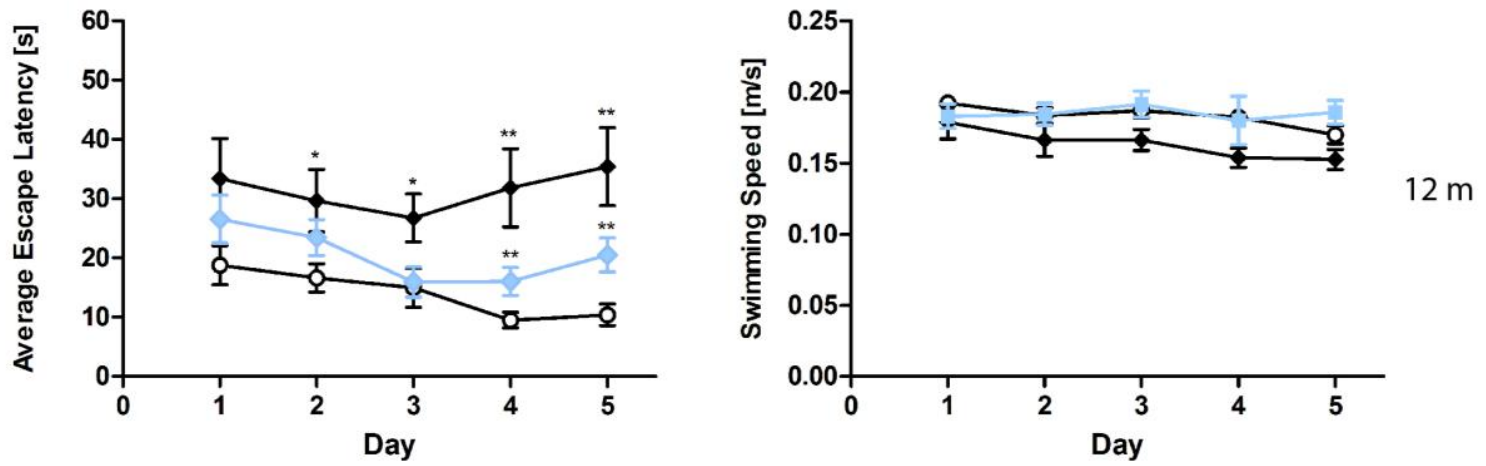

FIGURE 3.17 Spatial learning deficits in aged Tg4-42 and 5XFAD shown in the acquisition training of the Morris water maze. Female (A) 3- and (B) 12-month-old Tg4-42 mice, 5XFAD mice and WT littermate controls were tested. Animals tested underwent acquisition training to learn to use distal and proximal cues to navigate a direct path to a hidden platform. (A) Escape latencies of young mice decreased progressively over 5 days of training regardless of the genotype. Furthermore, (B) aged WT mice showed a progressive improvement in the escape latency over time. The escape latencies for aged Tg4-42 and 5XFAD did not improve over the five days of training. Swimming speed was not affected in all mice tested. Escape latency and swimming speed: two-way repeated measures ANOVA. ${ }^{* *} p<0.01 ;{ }^{*} p<0.05 . \mathrm{n}=8-11$ per group; data presented as mean \pm S.E.M. 
A
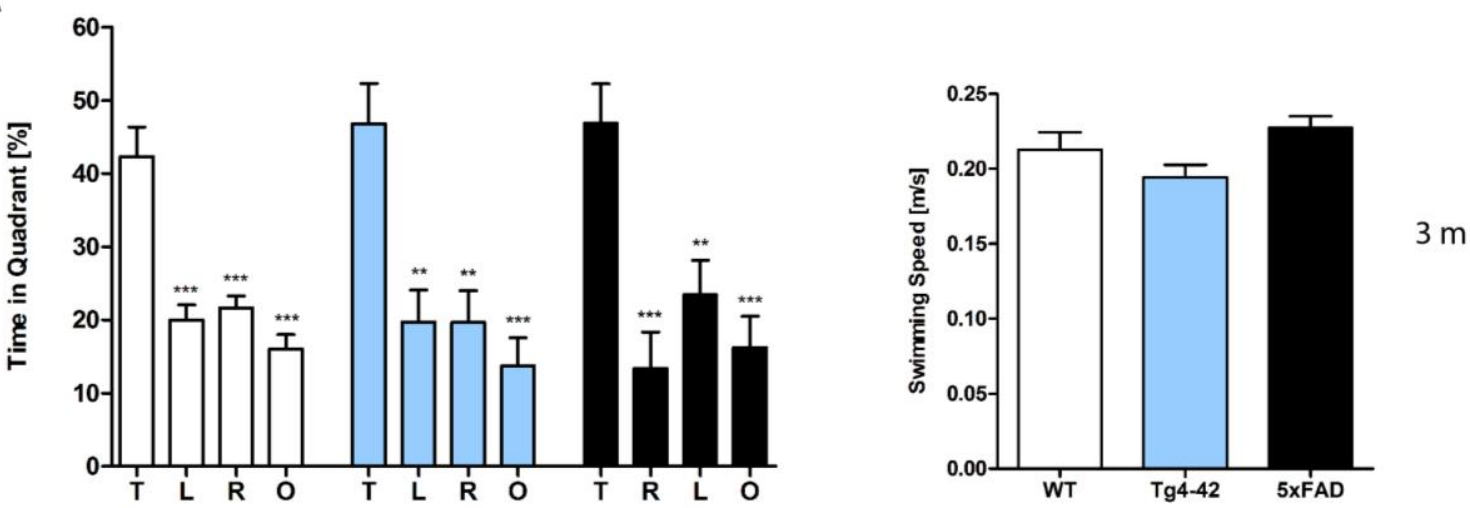

B
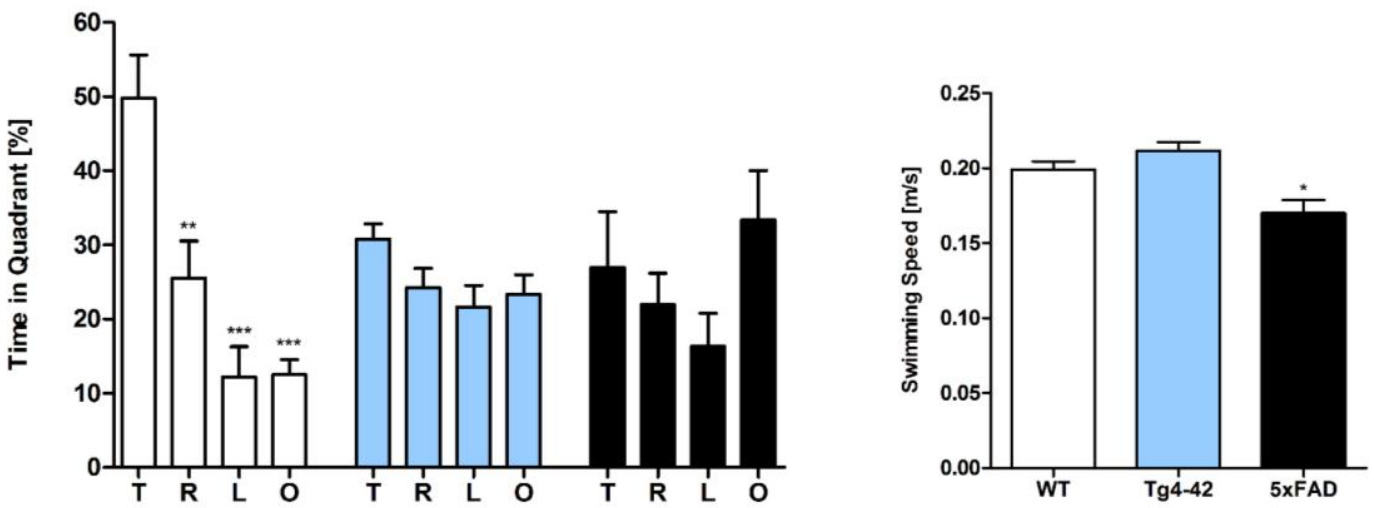

$12 \mathrm{~m}$

FIGURE 3.18 Spatial reference memory deficits in aged Tg4-42 and 5XFAD mice shown in the probe trial of the Morris water maze. Female 3- and 12-month-old Tg4-42 mice, 5XFAD mice and WT littermate controls were tested. The probe trial was given at the end of learning phase (acquisition training) to assess spatial reference memory. Quadrant preference and swimming speed for the first 30s of the probe trial were analyzed. (A) Young Tg4-42, 5XFAD and WT mice showed no impairment in spatial reference memory. All groups spent a significant greater percentage of time in the target quadrant. (WT: $p<0.001 \mathrm{~T}$ vs. all other quadrants; 5XFAD: $p<$ $0.001 \mathrm{~T}$ vs. $\mathrm{L}$ and $\mathrm{O}, p<0.01 \mathrm{~T}$ vs. $\mathrm{R} ; \mathrm{Tg} 4-42: p<0.001 \mathrm{~T}$ vs. $\mathrm{O}, p<0.01 \mathrm{~T}$ vs. $\mathrm{R}$ and $\mathrm{L}$ ) The swimming speed did not differ between the groups. (B) Probe trial revealed a significant reduced learning behavior for aged Tg4-42 and 5XFAD mice as they showed no preference for the target quadrant. WT mice have no learning deficits at this age (WT: $p<0.001 \mathrm{~T}$ vs. $L$ and $0, p<0.01 \mathrm{~T}$ vs. R). 5XFAD mice showed a reduced swimming speed during the probe trial. Abbreviations: $T=$ target quadrant; $L=$ left quadrant; $R=$ right quadrant; $O=$ opposite quadrant. Quadrant preference and swimming speed: One-way analysis of variance (ANOVA) followed by Bonferroni multiple comparisons. ${ }^{* *} p<0.001 ;{ }^{* *} p<0.01$. $\mathrm{n}=8-11$ per group; data presented as mean \pm S.E.M. 


\subsection{3}

Tg4-42 and 5XFAD mice exhibit decreased contextual learning

During the initial training sessions involving a tone-foot-shock pairing (CS/US), 12-monthold Tg4-42, 5XFAD and WT mice exhibited comparable degrees of freezing. When mice were tested for context fear conditioning 24 hours after the training trial, Tg4-42 and 5XFAD mice demonstrated no significantly increased freezing behavior as a response to the conditioning chamber (FIGURE 3.9). In contrast, WT mice displayed significantly increased freezing (one-way ANOVA, $p<0.001$ ) as a response to the context.

Tg4-42 and 5XFAD mice jumped and vocalized in response to the electric foot-shock to a similar degree as WT mice, suggesting normal pain perception in the mutant mice strains. However, transgenic mice were not able to attribute the pain of the foot-shock during the training trial to the context. Therefore, Tg4-42 and 5XFAD show impaired contextual learning.

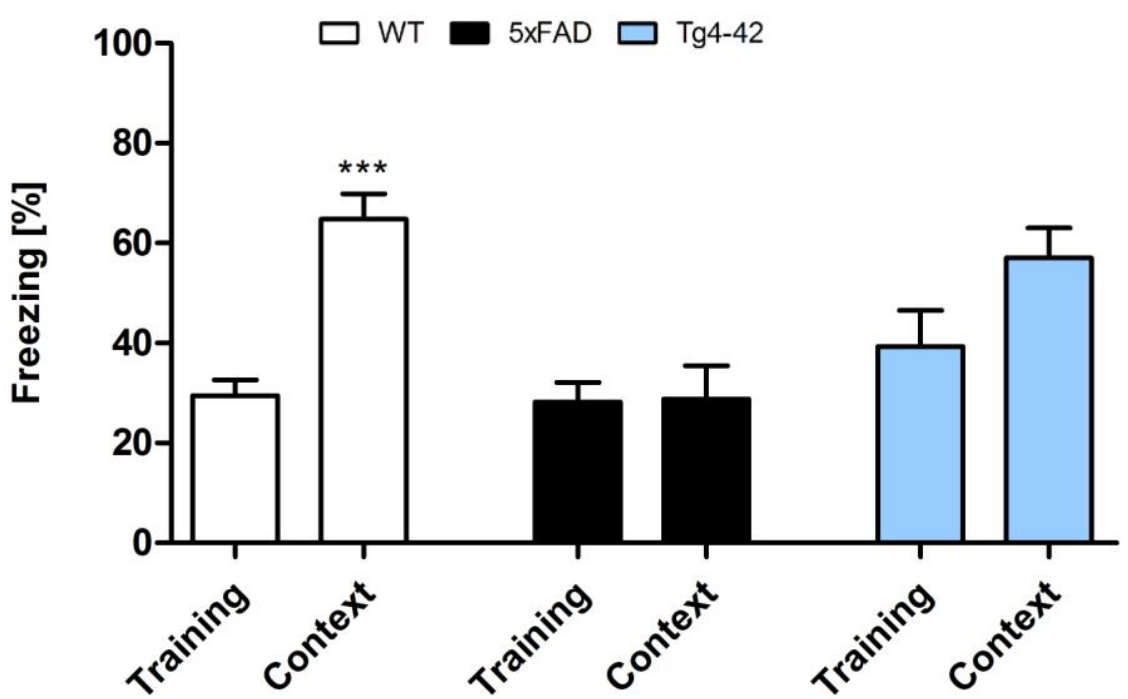

FIGURE 3.19 Impaired contextual conditioning in Tg4-42 and 5XFAD mice. Aged 5XFAD, Tg4-42 and WT mice were trained with a CS/US pairing for contextual fear conditioning. Mice were reintroduced to the original training context (CS) 24 hours post training and tested for contextual memory. Levels of freezing during the re-exposure were not different from the training trial for 5XFAD and Tg4-42. In contrast, WT mice showed a significant increase on freezing response to the context. $\mathrm{CS}=$ conditioned stimulus. Freezing: paired t-test; ${ }^{* * *} p<0.001 . \mathrm{n}=11-13$ per group; data presented as mean \pm S.E.M.

\section{2 .4}

\section{XFAD mice show impaired tone learning}

Twenty-four hours after the context testing (48 hours after training), the same mice were tested for conditioned fear of a tone. Therefore mice were reintroduced to the altered fear conditioning chamber. When the tone was presented without the foot-shock, both Tg4-42 and WT mice exhibited similar freezing responses (FIGURE 3.20). In both mouse lines 
freezing increased significantly compared to the pre-tone period (one-way ANOVA, WT and Tg4-42: $p<0.001)$. However, 5XFAD mice demonstrated substantially less freezing behavior in response to the tone. 5XFAD mice did not associate the tone with the previously received foot-shock as freezing was not significantly different between the training and the tone trial.

These results indicate that $\operatorname{Tg} 4-42$ mice exhibit a selective impairment of contextual fear learning, while their tone learning ability remains intact. 5XFAD mice on the other hand demonstrate both impaired contextual and tone fear learning.

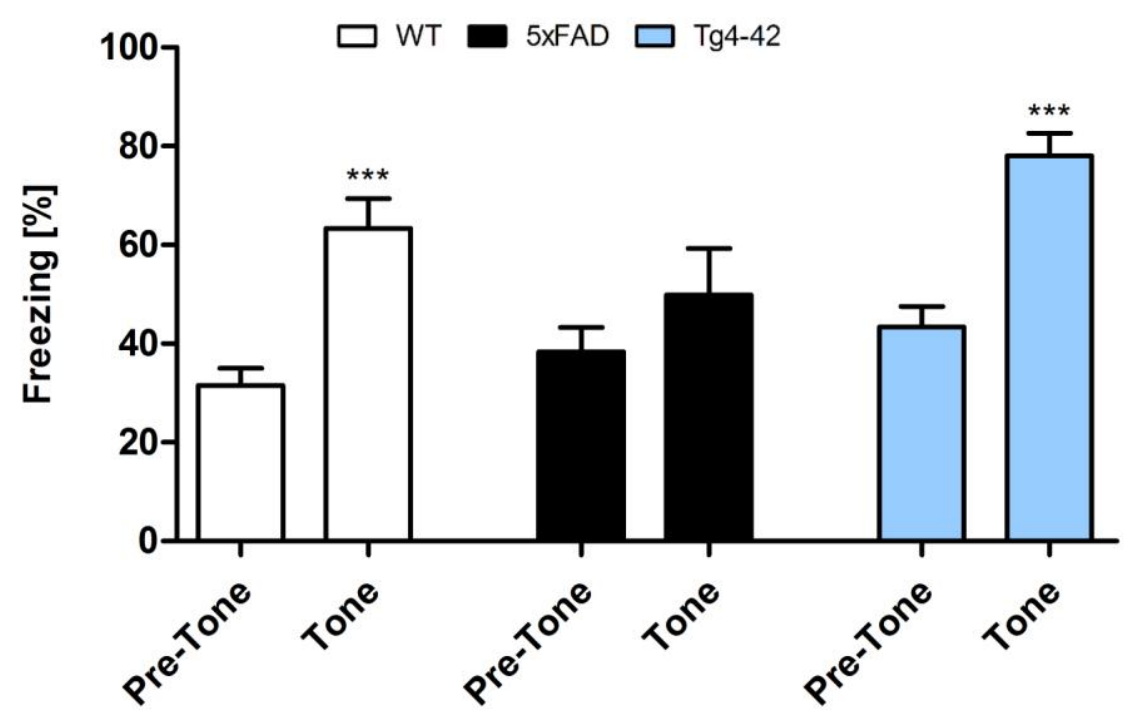

FIGURE 3.20 Impaired tone conditioning in 5XFAD mice Aged 5XFAD, Tg4-42 and WT mice at 12 months of age were trained with a CS/US pairing for tone fear conditioning. Mice were placed in an altered fear conditioning chamber 48 hours post training and tested for freezing during tone presentation (CS). WT and Tg4-42 mice shock froze significantly more during tone presentation compared to the training trial. In contrast, 5XFAD mice did not associate the tone with the received foot-shock as freezing was not significantly different between the training and the tone trial. $\mathrm{CS}=$ conditioned stimulus, $\mathrm{US}=$ unconditioned stimulus. Freezing: paired t-test; ${ }^{* * *} p<0.001 . \mathrm{n}=11-13$ per group; data presented as mean \pm S.E.M. 


\subsubsection{Deep sequencing of mouse brains}

In total, deep sequencing identified $15,711,910$ and $16,143,760$ sequence reads for young and old wild-type mice, respectively. For young wild-type mice, 6,230,197 reads (39.65\%) and for old wild-type mice, 5,512,056 reads (34.14\%) were mapped to exons. In young 5XFAD mice, the read mapping revealed 8,570,239 (60.28\%) of 14,216,258 reads in exonic regions. Out of 18,288,161 reads, 9,163,060 (50.10\%) hit exons in old 5XFAD mice. The brain exome of young Tg4-42 mice was covered by 6,342,018 (47.28 \%) out of 13,414,301 reads. For old Tg4-42 mice, 12,488,206 reads were detected in total, of which $4,976,552$ (39.85\%) could be mapped to exons. The numbers of exonic reads are summarized in TABLE 3.1.

TABLE 3.1 Number of exonic reads in brain tissue of wild-type and transgenic mice

\begin{tabular}{cc}
\hline Genotype & Number of reads in exons \\
\hline young WT & $6,230,197$ \\
aged WT & $5,512,056$ \\
young 5XFAD & $8,570,239$ \\
aged 5XFAD & $9,163,060$ \\
young Tg4-42 & $6,342,018$ \\
aged Tg4-42 & $4,976,552$ \\
\hline
\end{tabular}

3.2.5.1

Deep sequencing (RNASeq) identified over-expressed transgenes

5XFAD mice over-express human amyloid precursor protein (APP695) carrying the Swedish, London and Florida mutations as well as human presenilin-1 (PSEN-1) carrying the M146L/L286V mutations. Both peptides are expressed under the control of the neuronal Thy-1 promoter (Oakley et al., 2006). As expected, sequence reads pertaining to PSEN-1, APP and a Thy-1 promoter sequence (Moechars et al., 1996) were overrepresented in both young and aged 5XFAD brains (TABLE 3.2) and therefore served as a positive and internal control for RNA-Seq.

In Tg4-42 mice a Thy-1 promoter sequence (Moechars et al., 1996) was found to be over-expressed in both young and aged mice (TABLE 3.2). Again, this was expected as Tg4-42 mice express human $A \beta_{4-42}$ fused to the murine $T R H$ signal peptide under the control of the neuronal Thy-1 promoter (Bouter et al., 2013). 
TABLE 3.2 Over-expressed transgenes in 5XFAD and Tg4-42 mice identified through RNASeq

\begin{tabular}{cccc}
\hline Genotype & Gene name & Log2 fold change & Adjusted p-value \\
\hline young 5XFAD & App & 0.90 & $3.51 \mathrm{E}-02$ \\
young 5XFAD & Psen1 & 1.06 & $3.51 \mathrm{E}-02$ \\
young 5XFAD & Thy1 & 1.65 & $1.16 \mathrm{E}-08$ \\
aged 5XFAD & App & 0.90 & $5.41 \mathrm{E}-03$ \\
aged 5XFAD & Psen1 & 1.40 & $2.29 \mathrm{E}-05$ \\
aged 5XFAD & Thy1 & 2.91 & $2.45 \mathrm{E}-30$ \\
young Tg4-42 & Thy1 & 1.58 & $3.02 \mathrm{E}-08$ \\
aged Tg4-42 & Thy1 & 2.00 & $2.28 \mathrm{E}-14$ \\
\hline
\end{tabular}

\subsubsection{Differentially regulated genes in Tg4-42 and 5XFAD mice}

The total number of differentially regulated genes (DEG) in each mouse model is listed in TABLE 3.3 (excluding transgenes). Aged 5XFAD demonstrated the highest number of DEG followed by aged Tg4-42 and young 5XFAD. In contrast young Tg4-42 mice showed - except of the promoter sequence (section 3.2.5.1) - no genes that were significantly changed.

TABLE 3.3 Number of DEGs in 5XFAD and Tg4-42

\begin{tabular}{ccc}
\hline Genotype & Number of up-regulated genes & Number of down-regulated genes \\
\hline young 5XFAD & 13 & 6 \\
young Tg4-42 & - & - \\
aged 5XFAD & 62 & 69 \\
aged Tg4-42 & 49 & 7 \\
\hline
\end{tabular}

In order to demonstrate the expression changes volcano plots were created (FIGURE 3.21). Each dot represents one gene that is significantly differentially regulated when compared to same-aged WT mice. Red dots demonstrate down-regulated genes while green dots show up-regulated genes. 
A

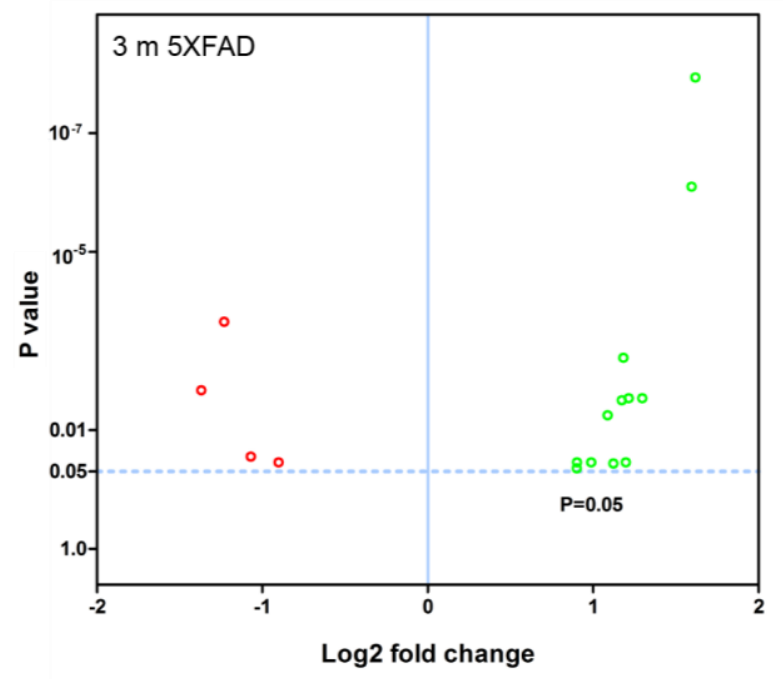

C

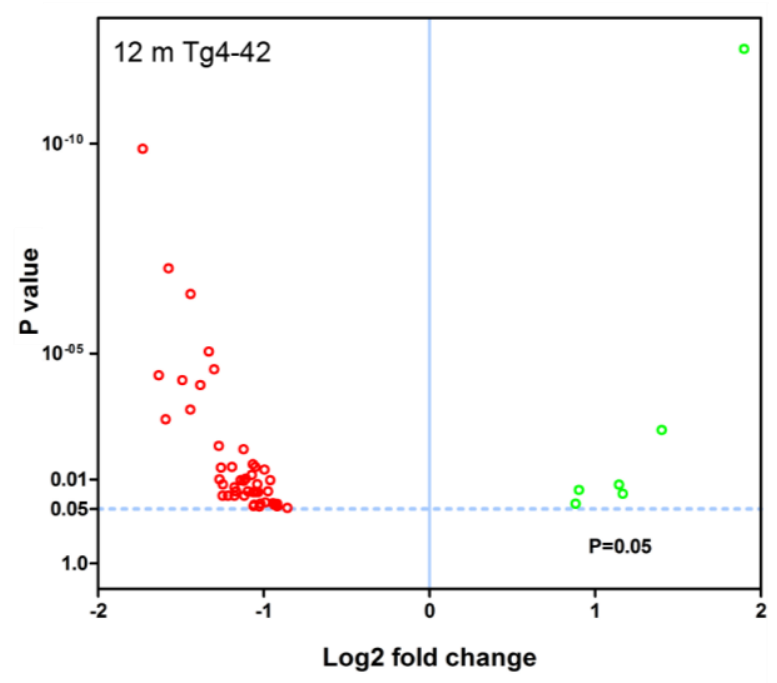

B

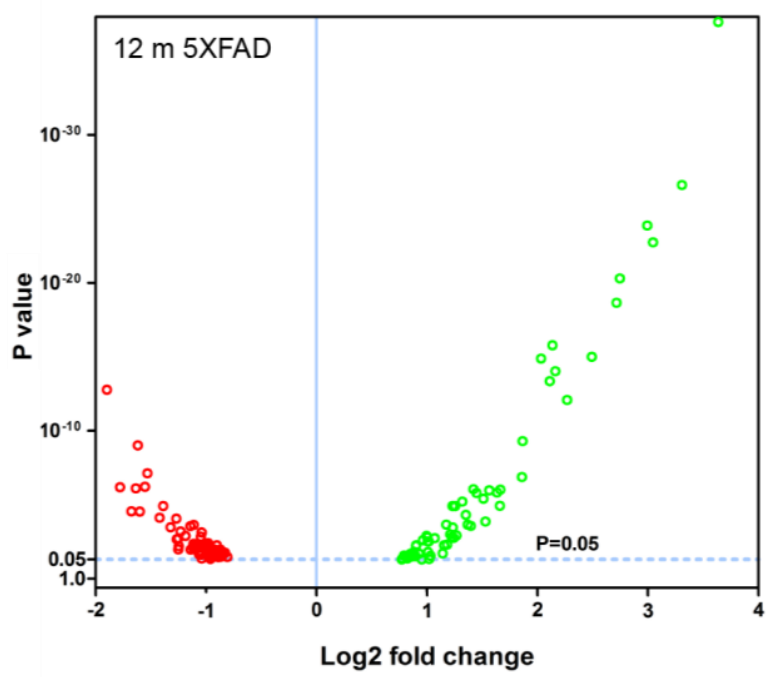

FIGURE 3.21 Volcano plots of the significant gene expression changes in Tg4-42 and 5XFAD mice. Fold changes in gene expression of (A) young 5XFAD, (B) aged 5XFAD and (C) aged Tg4-42 mice. Each dot represents one gene. Dashed lines illustrate statistical significance $(p=0.05)$. Red: down-regulated; green: up-regulated.

\subsubsection{Gene expression in young Tg4-42 and 5XFAD mice}

Nineteen genes were identified as significantly differentially expressed between young 5XFAD and age-matched WT mice. Thirteen genes were up-regulated, while six genes were down-regulated (FIGURE 3.21 A). DEGs encoded proteins from diverse functional categories, including translation (ribosomal proteins), glycolysis and ATP-binding, kinases 
and hydrolases (TABLE 3.4). In contrast, no differentially expressed genes could be detected in young $\mathrm{Tg} 4-42$ mice.

\subsubsection{Gene expression in aged Tg4-42 mice}

Fifty-six genes were differentially expressed in aged Tg4-42 mice. Seven genes were upregulated and 49 down-regulated (FIGURE $3.21 \mathrm{C}$ ). Twenty genes were solely differentially expressed in aged Tg4-42 (TABLE 3.5), among these only three genes were found to be up-regulated (Uqcc2, Beta-S and Kif1a). The 17 genes that were significantly down-regulated are involved in diverse biological processes including regulation of gene expression, nervous system development, cell communication, metal ion transport, neurogenesis and regulation of synaptic plasticity.

\subsubsection{Gene expression in aged 5XFAD mice}

In aged 5XFAD mice 131 genes with significant expression changes were identified. While 62 genes were up-regulated, 69 genes were down-regulated (FIGURE 3.21 B). 87 of the genes were only found to be altered in aged 5XFAD mice (TABLE 3.7), while 36 showed an overlap with aged $\mathrm{Tg} 4-42$ mice (TABLE 3.6) and eight were also differentially expressed in young 5XFAD mice (FIGURE 3.22).

According to the Gene Ontology (GO) annotation a notable group DEGs is involved in immune system processes and inflammation. These include, among others, innate immune response and adaptive immune response, immune effector processes, activation and regulation of immune response as well as immune system development. Furthermore, DEGs were also involved in cell communication and system development, signal transduction, synaptic transmission as well as regulation of gene expression and transcription.

\subsubsection{Genes similarly expressed in both aged Tg4-42 and 5XFAD mice}

Of the 56 DEGs in aged Tg4-42 mice, 36 were also found to be differentially expressed in aged 5XFAD mice (TABLE 3.6, FIGURE 3.22). Of these 36 genes four were up-regulated and 32 were down-regulated in aged Tg4-42 and 5XFAD mice and most showed similar expression levels in the two models.

The biggest differences between aged Tg4-42 and 5XFAD could be detected in the expression of Gfap and Xist. The intermediate filament protein GFAP encoding gene was found to be four times higher over-expressed in 5XFAD compared to Tg4-42. The non-protein coding RNA Xist was two-fold less abundant in Tg4-42 as compared to 
5XFAD mice. Apart from Gfap, Calmodulin 3, Fbxo2 and Gpm6a were also up-regulated in both aged mouse lines. The functional annotation of the jointly down-regulated genes includes the following GO (Ashburner et al., 2000) categories: regulation of cell differentiation and anatomical structure development, regulation of gene expression and transcription, histone modification, ion binding and protein methyltransferase activity, nervous system development and neurogenesis.

Two genes were similarly down-regulated in aged Tg4-42 and 5XFAD but also young 5XFAD animals (FIGURE 3.22). First, UbqIn2 which encodes a member of the ubiquilin family (Ubiquilin 2) that is involved in the protein degrading pathway as it regulates the degradation of ubiquitinated proteins (Ko et al., 2004). Second, the RNA binding protein neuro-oncological ventral antigen 2 encoding gene (Nova2).

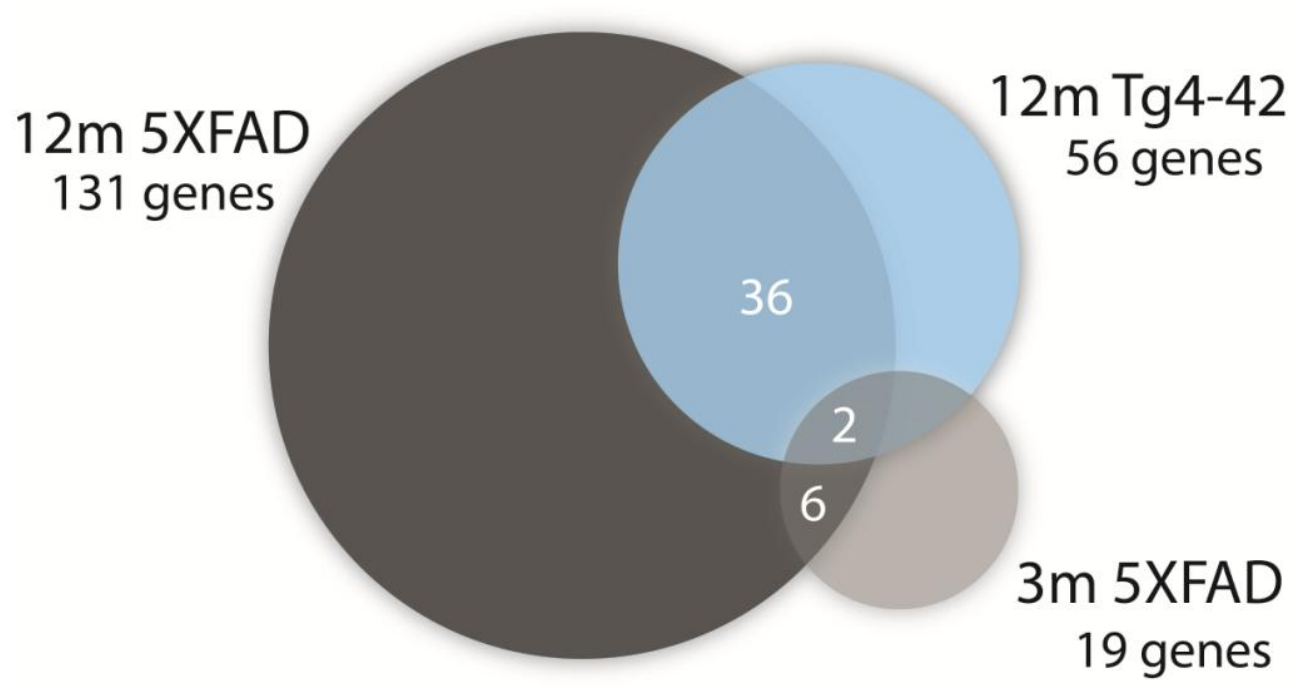

FIGURE 3.22 Venn diagram analysis for significantly regulated genes in Tg4-42 mice compared to 5XFAD mice. The numbers outside each circle represent the number of genes that were significantly differentially expressed in the respective mouse line (compared to WT mice). The numbers in the spaces of overlapping circles represent the number of genes that were affected in more than one condition. $p<0.05$.

\subsubsection{Genes similarly expressed in both young and aged 5XFAD mice}

Eight genes were found to be differentially expressed in both young and aged 5XFAD mice (FIGURE 3.22). Of these genes four were up-regulated and four down-regulated. The up-regulated genes are the ribosomal protein Rpl21, Aldolase A, Snora68 and the ribonuclease P RNA component H1. UbqIn2, Nova2, Atp1a2 and Rn45s showed reduced expression. 
3.2.5.8

Validation of differentially expressed genes identified by RNA-Seq using qRT-PCR

Deep sequencing results were validated through qRT-PCR analysis.

A

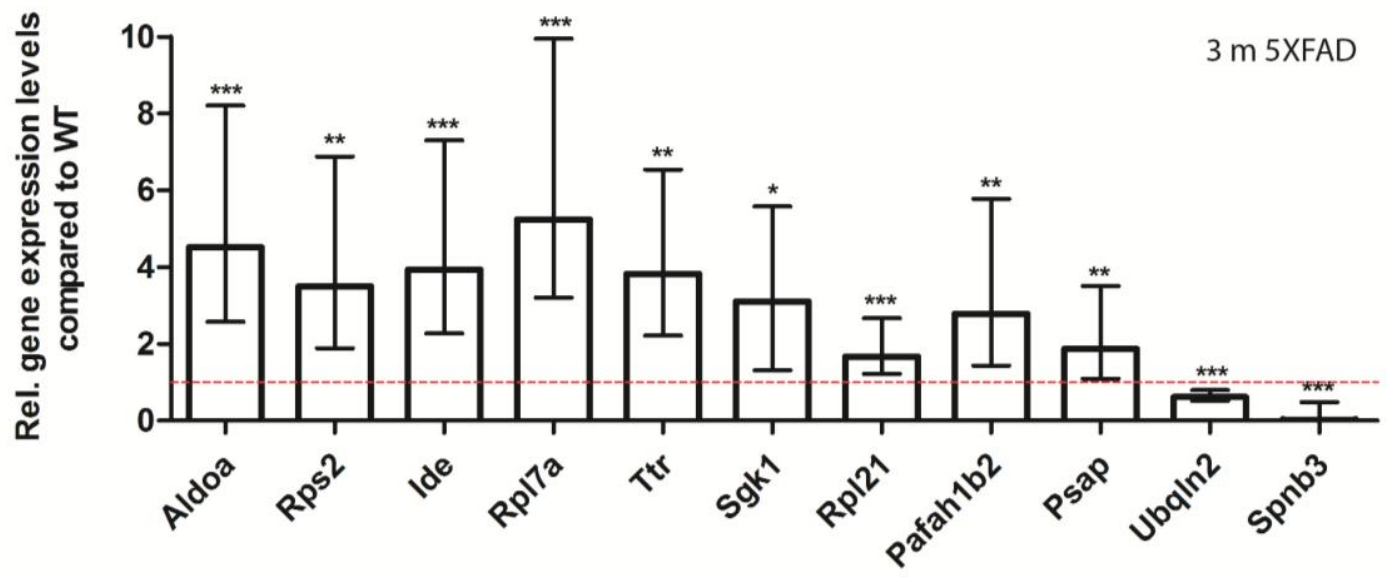

B

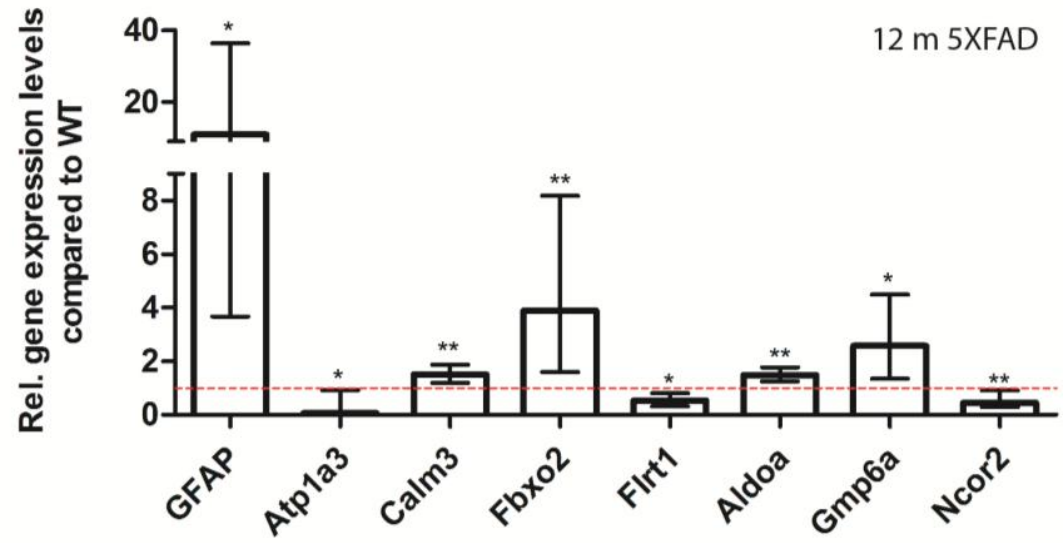

C

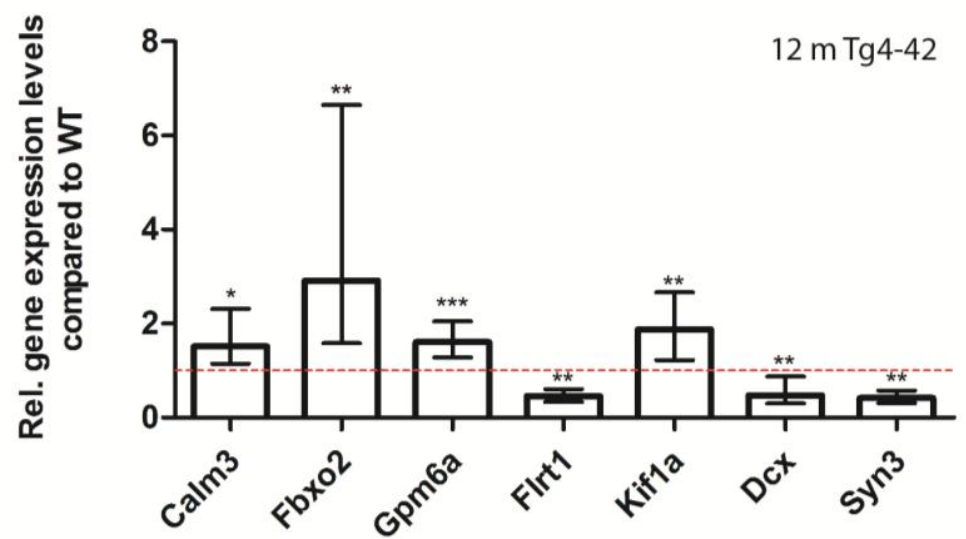

FIGURE 3.23 Validation of deep sequencing results through quantitative real-time polymerase chain reaction (qRT-PCR) analysis. To confirm the deep-sequencing data qRT-PCR experiments for various genes were performed on (A) young 5XFAD, (B) aged 5XFAD and (C) aged Tg4-42 mice. Expression levels of were compared to age-matched WT animals (dashed red line represents WT standard). Normalization was performed against the housekeeping gene $\beta$-Actin. ${ }^{* * *} p<0.001$; ${ }^{* *} p<0.01 ;{ }^{*} p<0.05 ; \mathrm{m}$ age in months; $\mathrm{n}=4-5$ per group; data presented as mean \pm S.E.M. 
The quality of the isolated RNA is crucial for obtaining reliable qRT-PCR results. Therefore, the quality of the RNA samples isolated from the mice brains was evaluated by assessing the integrity and purity of the RNA. All samples displayed A260/A230 ratios greater than 1.8 and A260/A280 ratios higher than 2.0 (data not shown) indicating an acceptable RNA purity.

Genes with more than 200 reads were successfully verified by real-time quantitative PCR and are listed in the following tables: TABLE 3.4, TABLE 3.5, TABLE 3.6 and TABLE 3.7. Genes with an expression level lower than 200 reads are not shown. For young 5XFAD (FIGURE 3.23 A), aged 5XFAD (FIGURE 3.23 B) and Tg4-42 (FIGURE $3.23 \mathrm{C})$ mice at least seven DEGs were randomly selected and validated using qRT-PCR. For all genes the qRT-PCR analysis revealed expression patterns similar to the deep sequencing results. 
TABLE 3.4 List of differentially expressed transcripts in young 5XFAD mice

\begin{tabular}{|c|c|c|c|c|c|}
\hline ID & $\begin{array}{l}\text { Gene } \\
\text { Name }\end{array}$ & Gene Description & $\begin{array}{l}\text { GO Biological Process } \\
\text { Annotation / functions }\end{array}$ & $\begin{array}{l}\text { log2 fold } \\
\text { change }\end{array}$ & $\begin{array}{l}\text { Adjusted } \\
\text { p-value }\end{array}$ \\
\hline MGI:87994 & Aldoa & $\begin{array}{l}\text { aldolase } A \text {, fructose- } \\
\text { bisphosphate }\end{array}$ & $\begin{array}{ll}\text { - } & \text { fructose-bisphosphate } \\
\text { aldolase activity } \\
\text { - } & \text { actin binding } \\
\text { - } & \text { cytoskeletal protein binding } \\
\text { - } & \text { tubulin binding } \\
\text { - } & \text { glycolysis }\end{array}$ & 1.62 & $1.16 \mathrm{E}-08$ \\
\hline MGI:2148181 & Snora68 & $\begin{array}{l}\text { small nucleolar RNA, H/ACA } \\
\text { box } 68\end{array}$ & $\begin{array}{ll}- & \text { non-coding RNA } \\
- & \text { uridine modifications }\end{array}$ & 1.60 & 7.99E-07 \\
\hline MGI:105110 & Rps2 & ribosomal protein S2 & $\begin{array}{ll}\text { - } & \text { mRNA binding } \\
\text { - } & \text { fibroblast growth factor } \\
\text { binding } \\
\text { - } \\
\text { structural constituent of } \\
\text { ribosome }\end{array}$ & 1.30 & $2.92 \mathrm{E}-03$ \\
\hline MGI:96412 & Ide & insulin degrading enzyme & $\begin{array}{ll}\text { - } & \text { insulysin activity } \\
\text { - } & \text { metalloendopeptidase } \\
\text { - } & \text { activity } \\
& \text { protein homodimerization } \\
\text { - } & \text { activity } \\
\text { - } & \text { hydrolase activity } \\
\text { - } & \text { geta-amyloid binding } \\
\text { - } & \text { ATP binding } \\
\text { - } & \text { zinc ion binding } \\
\text { - } & \text { ubiquitin binding }\end{array}$ & 1.22 & 2.92E-03 \\
\hline MGI:1353472 & $R p / 7 a$ & ribosomal protein $\mathrm{L} 7 \mathrm{a}$ & $\begin{array}{ll}- & \text { RNA binding } \\
\text { - } & \text { structural constituent of } \\
\text { ribosome }\end{array}$ & 1.20 & 3.51E-02 \\
\hline MGI:98865 & Ttr & transthyretin & $\begin{array}{ll}\text { - } & \text { hormone activity } \\
\text { - } & \text { protein heterodimerization } \\
\text { - } & \text { activity } \\
\text { retinol binding }\end{array}$ & 1.18 & $6.09 \mathrm{E}-04$ \\
\hline MGI:1340062 & Sgk1 & $\begin{array}{l}\text { serum/glucocorticoid regulated } \\
\text { kinase } 1\end{array}$ & $\begin{array}{ll}\text { - } & \text { kinase activity } \\
\text { - } & \text { potassium / calcium } \\
\text { channel regulator activity } \\
\text { - } & \text { ATP binding } \\
\text { - } & \text { response to DNA damage } \\
\text { stimulus }\end{array}$ & 1.17 & $3.16 \mathrm{E}-03$ \\
\hline MGI:1278340 & Rpl21 & ribosomal protein L21 & $\begin{array}{l}\text { - } \quad \begin{array}{l}\text { structural constituent of } \\
\text { ribosome }\end{array} \\
\text { - } \quad \text { RNA binding }\end{array}$ & 1.12 & 3.65E-02 \\
\hline MGl:108415 & Pafah1b2 & $\begin{array}{l}\text { platelet-activating factor } \\
\text { acetylhydrolase, isoform } 1 \mathrm{~b} \text {, } \\
\text { subunit } 2\end{array}$ & $\begin{array}{ll}\text { - } & \text { hydrolase activity } \\
\text { - } & \text { 1-alkyl-2 acetylglycero- } \\
\text { phosphocholine esterase } \\
\text { activity } \\
\text { - } & \text { homodimerization activity }\end{array}$ & 1.09 & 5.67E-03 \\
\hline MGI:99845 & Gdi2 & $\begin{array}{l}\text { guanosine diphosphate (GDP) } \\
\text { dissociation inhibitor } 2\end{array}$ & $\begin{array}{ll}\text { - } & \text { Rab GDP-dissociation } \\
\text { inhibitor activity } \\
\text { - } \quad \text { Rab GTPase activator } \\
\text { activity }\end{array}$ & 0.99 & 3.51E-02 \\
\hline MGI:1934664 & Rpph1 & $\begin{array}{l}\text { ribonuclease } \mathrm{P} \text { RNA } \\
\text { component } \mathrm{H} 1\end{array}$ & - $\quad$ endoribonuclease activity & 0.90 & 3.51E-02 \\
\hline MGI:97783 & Psap & prosaposin & $\begin{array}{ll}\text { - } & \text { glycoprotein } \\
\text { - } & \text { lipid binding } \\
\text { - } & \text { enzyme activator activity }\end{array}$ & 0.90 & 3.51E-02 \\
\hline MGI:97591 & $P k m$ & pyruvate kinase, muscle & $\begin{array}{ll}- & \text { magnesium ion binding } \\
- & \text { ATP binding } \\
\text { - } & \text { potassium ion binding } \\
& \text { pyruvate kinase activity }\end{array}$ & 0.90 & 4.41E-02 \\
\hline
\end{tabular}




\begin{tabular}{|c|c|c|c|c|c|}
\hline MGI:108391 & Kif1a & kinesin family member $1 \mathrm{~A}$ & $\begin{array}{ll}\text { - } & \text { ATP binding } \\
\text { - } & \text { phospholipid binding } \\
\text { - } & \text { motor activity } \\
\text { - } & \text { axonal neuropathies }\end{array}$ & -0.90 & $3.51 \mathrm{E}-02$ \\
\hline MGI:88106 & Atp1a2 & $\begin{array}{l}\text { ATPase, } \mathrm{Na}+\mathrm{K}+\text { transporting, } \\
\text { alpha } 2 \text { polypeptide }\end{array}$ & $\begin{array}{ll}\text { - } & \text { sodium:potassium- } \\
& \text { exchanging ATPase } \\
\text { activity } \\
\text { - } & \text { ATP binding } \\
\text { - } & \text { metal ion binding } \\
\text { - } & \text { hydrolase activity }\end{array}$ & -0.90 & $3.51 \mathrm{E}-02$ \\
\hline MGl:1860283 & Ubqln2 & ubiquilin 2 & $\begin{array}{l}\text { - } \quad \text { protein binding } \\
\text { - } \quad \text { cell death }\end{array}$ & -1.07 & 2.82E-02 \\
\hline MGI:1313261 & Spnb3 & $\begin{array}{l}\text { spectrin beta, non-erythrocytic } \\
2\end{array}$ & $\begin{array}{ll}\text { - } & \text { phospholipid binding } \\
\text { - } & \text { actin binding } \\
\text { - } & \text { structural constituent of } \\
& \text { cytoskeleton }\end{array}$ & -1.23 & $1.50 \mathrm{E}-04$ \\
\hline MGI:1337000 & $R n 45 s$ & 45S Pre-Ribosomal 5 & non-coding RNA & -1.23 & $1.50 \mathrm{E}-04$ \\
\hline MGI:104296 & Nova2 & $\begin{array}{l}\text { neuro-oncological ventral } \\
\text { antigen } 2\end{array}$ & - $\quad$ RNA binding & -1.37 & $2.14 \mathrm{E}-03$ \\
\hline
\end{tabular}


TABLE 3.5 List of transcripts exclusively expressed in aged Tg4-42 mice

\begin{tabular}{|c|c|c|c|c|c|}
\hline ID & $\begin{array}{l}\text { Gene } \\
\text { Name }\end{array}$ & Gene Description & $\begin{array}{l}\text { GO Biological Process } \\
\text { annotation / functions }\end{array}$ & $\begin{array}{l}\text { log2 fold } \\
\text { change }\end{array}$ & $\begin{array}{c}\text { Adjusted } \\
\text { p-value }\end{array}$ \\
\hline MGI:1914517 & Uqcc2 & $\begin{array}{l}\text { ubiquinol-cytochrome c } \\
\text { reductase complex assembly } \\
\text { factor } 2\end{array}$ & $\begin{array}{l}\text { - } \quad \text { regulation of insulin } \\
\text { secretion } \\
\text { - } \quad \text { ATP production }\end{array}$ & 1.17 & $2.18 \mathrm{E}-02$ \\
\hline MGI:5474852 & Beta-S & hemoglobin, beta adult s chain & $\begin{array}{ll}- & \text { iron ion binding } \\
\text { - } & \text { oxygen binding }\end{array}$ & 1.14 & $1.31 \mathrm{E}-02$ \\
\hline MGI:108391 & Kif1a & kinesin family member $1 \mathrm{~A}$ & $\begin{array}{l}\text { - } \quad \text { microtubule motor activity } \\
\text { - } \quad \text { ATP binding }\end{array}$ & 0.90 & $1.77 \mathrm{E}-02$ \\
\hline MGI:2153272 & Trrap & $\begin{array}{l}\text { transformation/transcription } \\
\text { domain-associated protein }\end{array}$ & $\begin{array}{l}\text { - } \quad \text { phosphotransferase activity } \\
\text { - } \quad \text { regulation of transcription }\end{array}$ & -0.86 & 4.74E-02 \\
\hline MGI:1194504 & Kcnj10 & $\begin{array}{l}\text { ATP-sensitive inward rectifier } \\
\text { potassium channel } 10\end{array}$ & $\begin{array}{l}\text { - } \quad \text { potassium channel activity } \\
\text { - } \quad \text { ATP binding }\end{array}$ & -0.93 & $3.76 \mathrm{E}-02$ \\
\hline MGI:3039582 & Lmtk3 & lemur tyrosine kinase 3 & $\begin{array}{l}\text { protein tyrosine kinase } \\
\text { activity }\end{array}$ & -0.94 & $3.98 \mathrm{E}-02$ \\
\hline MGI:1343180 & $V g f$ & nerve growth factor inducible & $\begin{array}{ll}\text { - } & \text { neuropeptide hormone } \\
\text { activity } \\
\text { - } \quad \text { synaptic plasticity } \\
\text { neurosecretory protein } \\
\text { (Jahn et al., 2011) } \\
\text { - } \quad \text { regulation of energy } \\
\text { balance (Jahn et al. 2011) } \\
\text { important for modulating } \\
\text { neuronal activity (Cocco et } \\
\text { al. 2010) }\end{array}$ & -0.95 & 3.64E-02 \\
\hline MGl:106374 & Zmiz2 & $\begin{array}{l}\text { zinc finger MIZ domain- } \\
\text { containing protein } 2\end{array}$ & $\begin{array}{l}\text { - } \quad \text { zinc ion binding } \\
\text { ligand-dependent nuclear } \\
\text { receptor transcription } \\
\text { coactivator activity }\end{array}$ & -0.98 & $1.90 \mathrm{E}-02$ \\
\hline MGI:1194488 & Slc32a1 & $\begin{array}{l}\text { Vesicular inhibitory amino acid } \\
\text { transporter } \\
\text { solute carrier family } 32 \text { (GABA } \\
\text { vesicular transporter), member } \\
1\end{array}$ & $\begin{array}{l}\text { - } \quad \text { glycine transporter activity } \\
\text { amino acid-polyamine } \\
\text { transporter activity } \\
\text { - } \quad \text { neurotransmitter transport }\end{array}$ & -1.02 & 3.76E-02 \\
\hline MGl:1277171 & $D c x$ & doublecortin & $\begin{array}{ll}\text { - } & \text { microtubule binding } \\
\text { - } & \text { protein kinase binding } \\
\text { neurogenesis }\end{array}$ & -1.03 & 4.42E-02 \\
\hline MGI:101947 & Hnrnpd & $\begin{array}{l}\text { heterogeneous nuclear } \\
\text { ribonucleoprotein D }\end{array}$ & $\begin{array}{ll}- & \text { regulation of transcription } \\
\text { - } & \text { RNA binding and telomeric } \\
\text { DNA binding }\end{array}$ & -1.04 & 1.99E-02 \\
\hline MGI:109591 & Nfic & nuclear factor I/C & $\begin{array}{l}\text { - } \quad \text { transcription factor activity } \\
\text { - } \quad \text { DNA binding }\end{array}$ & -1.10 & $1.90 \mathrm{E}-02$ \\
\hline MGI:2441726 & $B C 005537$ & cDNA sequence BC005537 & - unknown & -1.11 & $9.46 \mathrm{E}-03$ \\
\hline MGI:2673998 & Arhgap33 & $\begin{array}{l}\text { Rho GTPase activating protein } \\
33\end{array}$ & $\begin{array}{l}\text { - } \quad \text { Rac GTPase activator } \\
\text { activity } \\
\text { - } \quad \begin{array}{l}\text { phosphatidylinositol } \\
\text { binding }\end{array}\end{array}$ & -1.12 & $1.08 \mathrm{E}-02$ \\
\hline
\end{tabular}




\begin{tabular}{|c|c|c|c|c|c|}
\hline MGI:1330828 & Cdk5r2 & $\begin{array}{l}\text { Cyclin-dependent kinase } 5 \\
\text { activator } 2 \text { (p39) }\end{array}$ & $\begin{array}{ll}\text { - } & \text { ipid binding } \\
\text { - } & \text { cyclin-dependent protein } \\
\text { kinase } 5 \\
\text { - } & \text { activator activity } \\
\text { - } & \text { neuron-specific }\end{array}$ & -1.14 & 1.04E-02 \\
\hline MGI:2674092 & Zfp609 & zinc finger protein 609 & - $\quad$ zinc ion binding & -1.17 & $1.90 \mathrm{E}-02$ \\
\hline MGI:1915454 & $\begin{array}{l}2900060 \\
\text { B14Rik }\end{array}$ & $\begin{array}{l}\text { RIKEN cDNA } 2900060 \text { B14 } \\
\text { gene }\end{array}$ & unknown & -1.18 & $2.43 \mathrm{E}-02$ \\
\hline MGI:1351334 & Syn3 & synapsin III & $\begin{array}{l}\text { - } \quad \text { catalytic activity } \\
\text { - } \quad \text { ATP binding }\end{array}$ & -1.25 & $1.30 \mathrm{E}-02$ \\
\hline MGI:1920907 & Fbrs/1 & fibrosin-like 1 & - unknown & -1.25 & 2.43E-02 \\
\hline MGI:106589 & Hivep3 & $\begin{array}{l}\text { human immunodeficiency } \\
\text { virus type I enhancer binding } \\
\text { protein } 3\end{array}$ & $\begin{array}{ll}- & \text { DNA binding } \\
- & \text { zinc ion binding } \\
\text { - } & \text { transcription factor }\end{array}$ & -1.26 & $5.18 \mathrm{E}-03$ \\
\hline
\end{tabular}


TABLE 3.6 List of transcripts differentially expressed in both aged Tg4-42 and 5XFAD mice

\begin{tabular}{|c|c|c|c|c|c|c|c|}
\hline ID & $\begin{array}{l}\text { Gene } \\
\text { Name }\end{array}$ & Gene Description & $\begin{array}{l}\text { GO Biological Process } \\
\text { annotation / functions }\end{array}$ & $\begin{array}{c}\text { log2 } \\
\text { fold } \\
\text { change } \\
\text { Tg4-42 }\end{array}$ & $\begin{array}{l}\text { Adjusted } \\
\text { p-value } \\
\text { Tg4-42 }\end{array}$ & $\begin{array}{c}\text { log2 } \\
\text { fold } \\
\text { change } \\
\text { 5XFAD }\end{array}$ & $\begin{array}{l}\text { Adjusted } \\
\text { p-value } \\
\text { 5XFAD }\end{array}$ \\
\hline MGI:103249 & Calm3 & calmodulin 3 & $\begin{array}{ll}- & \text { ion channel binding } \\
- & \text { calcium ion binding } \\
\text { - } & \text { G-protein coupled receptor } \\
\text { protein signaling pathway }\end{array}$ & 1.90 & $5.49 \mathrm{E}-13$ & 1.00 & $1.28 \mathrm{E}-03$ \\
\hline MGl:2446216 & Fbxo2 & F-box protein 2 & $\begin{array}{ll}\text { - } & \text { ubiquitin-protein ligase } \\
\text { - } & \text { activity } \\
\text { - } & \text { glycoprotein binding } \\
\text { - } & \text { carbohydramyloid binding } \\
& \text { carbo binding }\end{array}$ & 1.40 & $6.60 \mathrm{E}-04$ & 1.24 & $1.73 \mathrm{E}-03$ \\
\hline MGI:107671 & Gpm6a & $\begin{array}{l}\text { neuronal membrane } \\
\text { glycoprotein M6-a }\end{array}$ & $\begin{array}{ll}\text { - } & \text { calcium channel activity } \\
\text { involved in neuronal } \\
\text { - } & \text { differentiation } \\
\text { role in neuronal plasticity }\end{array}$ & 0.90 & 1.77E-02 & 0.90 & $5.39 \mathrm{E}-03$ \\
\hline MGI:95697 & Gfap & glial fibrillary acidic protein & $\begin{array}{ll}\text { - } & \text { integrin binding } \\
\text { - } & \text { kinase binding } \\
\text { - } & \text { structural constituent of } \\
\text { cytoskeleton }\end{array}$ & 0.88 & 3.76E-02 & 3.64 & $2.36 \mathrm{E}-42$ \\
\hline MGI:1860283 & Ubqln2 & ubiquilin 2 & $\begin{array}{ll}\text { - } & \text { ubiquitin-binding } \\
\text { - } & \text { protein modification } \\
\text { - } & \text { proteolysis }\end{array}$ & -0.92 & 4.35E-02 & -1.27 & $8.71 \mathrm{E}-05$ \\
\hline MGI:97495 & $P b \times 1$ & $\begin{array}{l}\text { pre-B-cell leukemia } \\
\text { transcription factor } 1 \text { / pre B } \\
\text { cell leukemia homeobox } 1\end{array}$ & $\begin{array}{l}\text { - } \quad \text { transcription factor activity } \\
\text { protein heterodimerization } \\
\text { activity } \\
\text { involved in the regulation of } \\
\text { osteogenesis } \\
\text { required for skeletal } \\
\text { patterning and } \\
\text { programming }\end{array}$ & -0.92 & 3.79E-02 & -0.86 & $2.85 \mathrm{E}-02$ \\
\hline MGI:3647820 & Gm15800 & predicted gene 15800 & $\begin{array}{l}\text { - ubiquitin-protein ligase } \\
\text { activity }\end{array}$ & -0.96 & $1.04 \mathrm{E}-02$ & -0.83 & $1.84 \mathrm{E}-02$ \\
\hline MGI:96669 & Kcnc3 & $\begin{array}{l}\text { potassium voltage gated } \\
\text { channel, shaw-related } \\
\text { subfamily, member } 3\end{array}$ & $\begin{array}{l}\text { - } \quad \text { voltage-gated potassium } \\
\text { channel activity } \\
\text { delayed rectifier potassium } \\
\text { channel activity }\end{array}$ & -0.99 & 3.47E-02 & -1.04 & $6.53 E-03$ \\
\hline MGI:96828 & Lrp1 & $\begin{array}{l}\text { low density lipoprotein } \\
\text { receptor-related protein } 1\end{array}$ & $\begin{array}{ll}\text { - } & \text { endocytic receptor or } \\
\text { - } & \text { receptor activity } \\
\text { - } & \text { lipoprotein binding } \\
\text { - } & \text { calcium ion binding } \\
\text { - } & \text { beta-amoprotein binding } \\
\text { - } & \text { apoptotic cell clearance }\end{array}$ & -1.00 & $5.80 \mathrm{E}-03$ & -0.83 & 1.77E-02 \\
\hline MGI:2183691 & Nav2 & neuron navigator 2 & $\begin{array}{ll}\text { - } & \text { heparin binding } \\
\text { - } & \text { helicase activity } \\
\text { - } & \text { ATP binding } \\
\text { role in neuronal } \\
\text { development }\end{array}$ & -1.04 & $1.29 \mathrm{E}-02$ & -0.89 & 2.33E-02 \\
\hline MGI:1890563 & Wasf1 & WAS protein family, member 1 & - $\quad$ actin binding & -1.05 & $1.99 \mathrm{E}-02$ & -0.95 & $2.11 \mathrm{E}-02$ \\
\hline MGI:96995 & MII1 & $\begin{array}{l}\text { lysine }(\mathrm{K}) \text {-specific } \\
\text { methyltransferase } 2 \mathrm{~A}\end{array}$ & $\begin{array}{ll}- & \text { calcium ion binding } \\
\text { - } & \text { zinc ion binding } \\
\text { - } & \text { chromatin binding } \\
\text { - } & \text { histone methyltransferase } \\
\text { - } & \text { activity } \\
& \text { regulation of transcription }\end{array}$ & -1.05 & 4.97E-03 & -0.81 & 3.37E-02 \\
\hline MGI:2446229 & Tet3 & tet methylcytosine & $\begin{array}{l}\text { - methylcytosine } \\
\text { dioxygenase activity }\end{array}$ & -1.05 & $1.98 \mathrm{E}-02$ & -1.05 & $6.56 \mathrm{E}-03$ \\
\hline
\end{tabular}




\begin{tabular}{|c|c|c|c|c|c|c|c|}
\hline & & dioxygenase 3 & $\begin{array}{ll}\text { - } & \text { oxidoreductase activity } \\
\text { - } & \text { metal ion binding } \\
& \text { methylation process }\end{array}$ & & & & \\
\hline MGI:99948 & Zfh $\times 3$ & zinc finger homeobox 3 & $\begin{array}{ll}\text { - } & \text { GTP binding } \\
\text { sequence-specific DNA } \\
\text { binding transcription factor } \\
\text { activity } \\
\text { - } \\
\text { zinc ion binding }\end{array}$ & -1.06 & $3.98 \mathrm{E}-02$ & -1.12 & $7.24 \mathrm{E}-03$ \\
\hline MGl:1347464 & Foxg1 & forkhead box G1 & $\begin{array}{ll}\text { - } & \text { sequence-specific DNA } \\
\text { binding } \\
\text { negative regulation of } \\
\text { neuron differentiation } \\
\text { - } & \text { regulation of transcription } \\
\text { brain development } \\
\text { forebrain marker (Yahata et } \\
\text { al. 2011) }\end{array}$ & -1.06 & 4.30E-02 & -1.11 & $9.22 \mathrm{E}-03$ \\
\hline MGl:1919847 & Auts2 & $\begin{array}{l}\text { autism susceptibility candidate } \\
2\end{array}$ & - unknown & -1.07 & $2.00 \mathrm{E}-02$ & -1.05 & 7.45E-03 \\
\hline MGl:1915467 & Prrc2a & proline-rich coiled-coil 2A & - unknown & -1.07 & 4.33E-03 & -0.85 & 2.24E-02 \\
\hline MGl:1917685 & Inf2 & $\begin{array}{l}\text { inverted formin, } \mathrm{FH} 2 \text { and } \mathrm{WH} 2 \\
\text { domain containing }\end{array}$ & $\begin{array}{l}\text { - } \quad \text { Rho GTPase binding } \\
\text { actin binding }\end{array}$ & -1.07 & 7.70E-03 & -0.87 & 2.60E-02 \\
\hline MGl:2158663 & Inpp5j & $\begin{array}{l}\text { inositol polyphosphate 5- } \\
\text { phosphatase J }\end{array}$ & $\begin{array}{ll}\text { - } & \mathrm{SH} 3 \text { domain binding } \\
\text { - } & \text { hydrolase activity } \\
\text { - } & \text { phosphatase activity }\end{array}$ & -1.12 & 2.43E-02 & -1.78 & $6.55 \mathrm{E}-07$ \\
\hline MGI:2682319 & MII2 & $\begin{array}{l}\text { lysine (K)-specific } \\
\text { methyltransferase 2D }\end{array}$ & - histone methyltransferase & -1.12 & $1.90 \mathrm{E}-03$ & -1.05 & $1.48 \mathrm{E}-03$ \\
\hline MGI:3026647 & Flrt1 & $\begin{array}{l}\text { fibronectin leucine rich } \\
\text { transmembrane protein } 1\end{array}$ & $\begin{array}{l}\text { - receptor signaling protein } \\
\text { activity }\end{array}$ & -1.18 & $1.53 \mathrm{E}-02$ & -1.25 & $1.89 \mathrm{E}-03$ \\
\hline MGl:1888520 & Brd4 & bromodomain containing 4 & DNA binding & -1.19 & 4.97E-03 & -1.05 & $7.64 \mathrm{E}-03$ \\
\hline MGI:1916205 & Srrm4 & $\begin{array}{l}\text { serine/arginine repetitive } \\
\text { matrix } 4\end{array}$ & $\begin{array}{l}\text { - } \quad \text { mRNA binding } \\
\text { promotes alternative } \\
\text { splicing and inclusion of } \\
\text { neural-specific exons in } \\
\text { target mRNAs }\end{array}$ & -1.22 & 2.43E-02 & -1.25 & $6.20 \mathrm{E}-03$ \\
\hline MGl:1926106 & Fam163b & $\begin{array}{l}\text { family with sequence similarity } \\
163 \text {, member B }\end{array}$ & unknown & -1.27 & $9.85 \mathrm{E}-03$ & -1.60 & 2.91E-05 \\
\hline MGI:2685951 & Myo16 & myosin XVI & $\begin{array}{ll}\text { - } & \text { motor activity } \\
\text { - } & \text { ATP binding } \\
& \text { protein phosphatase } \\
& \text { binding }\end{array}$ & -1.27 & $1.57 \mathrm{E}-03$ & -1.09 & $4.62 \mathrm{E}-03$ \\
\hline MGl:1923304 & Prrc2b & proline-rich coiled-coil 2B & unknown & -1.30 & 2.37E-05 & -1.11 & $2.28 \mathrm{E}-04$ \\
\hline MGI:1923206 & Srrm2 & $\begin{array}{l}\text { serine/arginine repetitive } \\
\text { matrix } 2\end{array}$ & $\begin{array}{ll}\text { - } & \mathrm{C}_{2} \mathrm{H}_{2} \text { zinc finger domain } \\
\text { - } & \text { binding } \\
\text { - } & \text { RNA binding } \\
\text { involved in pre-mRNA } \\
\text { splicing }\end{array}$ & -1.33 & $8.92 E-06$ & -1.04 & 7.25E-04 \\
\hline MGl:1337080 & Ncor2 & $\begin{array}{l}\text { nuclear receptor co-repressor } \\
2\end{array}$ & $\begin{array}{ll}\text { - } & \text { chromatin binding } \\
\text { - } & \text { regulation of transcription } \\
\text { transcription corepressor } \\
\text { - } & \text { activity } \\
\quad \text { Notch binding }\end{array}$ & -1.38 & 5.62E-05 & -1.39 & $1.21 \mathrm{E}-05$ \\
\hline MGl:1306776 & Mtap1a & $\begin{array}{l}\text { microtubule-associated protein } \\
1 \mathrm{~A}\end{array}$ & $\begin{array}{ll}\text { - } & \text { structural molecule activity } \\
\text { - } & \text { microtubule assembly } \\
\text { perception of sound }\end{array}$ & -1.44 & 3.82E-07 & -1.62 & $9.68 \mathrm{E}-10$ \\
\hline
\end{tabular}




\begin{tabular}{|c|c|c|c|c|c|c|c|}
\hline MGI:104725 & Atn1 & atrophin 1 & $\begin{array}{l}\text { - } \quad \text { toxin receptor binding } \\
\text { transcription corepressor } \\
\text { activity }\end{array}$ & -1.44 & 2.15E-04 & -1.32 & 3.38E-04 \\
\hline MGI:104296 & Nova2 & $\begin{array}{l}\text { neuro-oncological ventral } \\
\text { antigen } 2\end{array}$ & - $\quad$ RNA binding & -1.49 & 4.27E-05 & -1.64 & 7.71E-07 \\
\hline MGI:3613677 & Shank1 & $\begin{array}{l}\text { SH3 and multiple ankyrin } \\
\text { Repeat Domains } 1\end{array}$ & $\begin{array}{ll}\text { - } & \text { SH3 domain binding } \\
\text { - } & \text { identical protein binding } \\
\text { - } & \text { synapse maturation }\end{array}$ & -1.57 & $9.26 \mathrm{E}-08$ & -1.53 & 7.43E-08 \\
\hline MGI:2679002 & Prr12 & proline rich 12 & DNA binding & -1.59 & $3.68 \mathrm{E}-04$ & -1.68 & $2.80 \mathrm{E}-05$ \\
\hline MGI:2143886 & Dot1I & $\begin{array}{l}\text { DOT1-like histone } \mathrm{H} 3 \\
\text { methyltransferase }\end{array}$ & $\begin{array}{ll}- & \text { transcription factor binding } \\
- & \text { DNA binding } \\
- & \text { histone-lysine } \mathrm{N}- \\
& \text { methyltransferase activity }\end{array}$ & -1.63 & $3.29 E-05$ & -1.06 & $1.72 \mathrm{E}-02$ \\
\hline MGI:88107 & Atp1a3 & $\begin{array}{l}\text { sodium/potassium- } \\
\text { transporting ATPase subunit } \\
\text { alpha-3 }\end{array}$ & $\begin{array}{ll}\text { - } & \text { sodium:potassium- } \\
\text { exchanging ATPase } \\
\text { activity } \\
\text { - } \quad \text { ATP binding } \\
\text { - } \quad \text { metal ion binding } \\
\text { - } & \text { hydrolase activity }\end{array}$ & -1.73 & $1.31 \mathrm{E}-10$ & -1.90 & $1.70 \mathrm{E}-13$ \\
\hline MGI:98974 & Xist & inactive $X$ specific transcripts & - $\quad$ non-protein coding & -1.73 & $1.31 \mathrm{E}-10$ & -0.90 & $5.70 \mathrm{E}-03$ \\
\hline
\end{tabular}


TABLE 3.7 List of differentially expressed transcripts in aged 5XFAD mice

\begin{tabular}{|c|c|c|c|c|c|}
\hline ID & $\begin{array}{l}\text { Gene } \\
\text { Name }\end{array}$ & Gene Description & $\begin{array}{l}\text { GO Biological Process } \\
\text { annotation / functions }\end{array}$ & $\begin{array}{l}\text { log2 fold } \\
\text { change }\end{array}$ & $\begin{array}{c}\text { Adjusted } \\
\text { p-value }\end{array}$ \\
\hline MGI:88228 & $c 4 b$ & complement component 4B & $\begin{array}{ll}\text { - } & \text { endopeptidase inhibitor } \\
\text { - } & \text { activity } \\
\text { - } & \text { Inflammatory response } \\
\text { - } & \text { immunement activation } \\
& \text { immesponse }\end{array}$ & 3.31 & 2.44E-27 \\
\hline MGI:88225 & C1qc & $\begin{array}{l}\text { complement component } 1 . q \\
\text { subcomponent. } \mathrm{C} \text { chain }\end{array}$ & $\begin{array}{l}\text { - } \quad \text { complement activation } \\
\text { immune response }\end{array}$ & 3.05 & $1.84 \mathrm{E}-23$ \\
\hline MGI:88223 & C1qa & $\begin{array}{l}\text { complement component } 1 . q \\
\text { subcomponent. alpha } \\
\text { polypeptide }\end{array}$ & $\begin{array}{ll}\text { - } & \text { phosphate transport } \\
\text { - } & \text { complement activation } \\
\text { - } & \text { immune response }\end{array}$ & 2.99 & $1.31 \mathrm{E}-24$ \\
\hline MGI:107341 & Ctss & cathepsin S & $\begin{array}{ll}\text { - } & \text { cysteine-type peptidase } \\
\text { - } & \text { hydivity } \\
\text { - } & \text { proteolysis } \\
\text { - } & \text { immune response }\end{array}$ & 2.75 & $5.00 \mathrm{E}-21$ \\
\hline MGI:88224 & $C 1 q b$ & $\begin{array}{l}\text { complement component } 1 . q \\
\text { subcomponent. beta } \\
\text { polypeptide }\end{array}$ & $\begin{array}{ll}\text { - } & \text { phosphate transport } \\
\text { - } & \text { complement activation } \\
\text { - } & \text { immune response }\end{array}$ & 2.72 & $2.22 \mathrm{E}-19$ \\
\hline MGI:1891190 & Ctsz & cathepsin Z & $\begin{array}{l}\text { - } \quad \text { cysteine-type peptidase } \\
\text { activity } \\
\text { - } \quad \text { hydrolase activity }\end{array}$ & 2.49 & $1.01 \mathrm{E}-15$ \\
\hline MGI:98932 & $\operatorname{Vim}$ & vimentin & $\begin{array}{ll}\text { - } & \text { structural constituent of } \\
\text { cytoskeleton } \\
\text { - } \quad \text { identical protein binding } \\
\text { - } \quad \text { apoptotic process }\end{array}$ & 2.27 & 8.36E-13 \\
\hline MGI:96074 & Hexb & hexosaminidase $B$ & $\begin{array}{l}\text { - } \quad \text { cation binding } \\
\text { protein homodimerization } \\
\text { activity } \\
\text { Beta- } \mathrm{N} \text { - } \\
\text { acetylhexosaminidase } \\
\text { activity } \\
\text { protein heterodimerization } \\
\text { activity }\end{array}$ & 2.16 & $9.49 \mathrm{E}-15$ \\
\hline MGI:88562 & Ctsd & cathepsin D & $\begin{array}{ll}\text { - } & \text { aspartic-type } \\
\text { endopeptidase activity } \\
\text { - } \quad \text { hydrolase activity }\end{array}$ & 2.13 & $1.66 \mathrm{E}-16$ \\
\hline MGI:2148181 & Snora68 & $\begin{array}{l}\text { small nucleolar RNA. H/ACA } \\
\text { box } 68\end{array}$ & $\begin{array}{ll}- & \text { non-coding RNA } \\
-\quad & \text { uridine modifications }\end{array}$ & 2.11 & 4.37E-14 \\
\hline MGI:87994 & Aldoa & $\begin{array}{l}\text { aldolase A. fructose- } \\
\text { bisphosphate }\end{array}$ & $\begin{array}{ll}\text { - } & \text { actin binding } \\
\text { - } & \text { fructose-bisphosphate } \\
& \text { aldolase activity } \\
\text { - } & \text { cytoskeletal protein binding } \\
\text { - } & \text { tubulin binding } \\
\text { - } & \text { glycolysis }\end{array}$ & 2.03 & $1.32 \mathrm{E}-15$ \\
\hline MGI:88127 & $B 2 m$ & beta-2 microglobulin & $\begin{array}{l}\text { - } \quad \text { MHC class I receptor } \\
\text { - } \quad \text { cellivity } \\
\text { - } \quad \text { innate immune response }\end{array}$ & 1.86 & $5.03 \mathrm{E}-10$ \\
\hline MGI:108046 & Laptm5 & $\begin{array}{l}\text { Iysosomal-associated protein } \\
\text { transmembrane } 5\end{array}$ & - transmembran transport & 1.86 & $1.32 \mathrm{E}-07$ \\
\hline
\end{tabular}




\begin{tabular}{|c|c|c|c|c|c|}
\hline MGI:1333815 & $C \times 3 c r 1$ & $\begin{array}{l}\text { chemokine (C-X3-C motif) } \\
\text { receptor } 1\end{array}$ & $\begin{array}{ll}\text { - } & \text { chemokine receptor activity } \\
\text { - } & \text { activitein coupled receptor } \\
\text { - } & \text { transmembrane protein } \\
\text { - } & \text { signal transduction }\end{array}$ & 1.66 & $9.43 E-07$ \\
\hline MGI:99554 & Lgals3bp & $\begin{array}{l}\text { lectin. galactoside-binding. } \\
\text { soluble. } 3 \text { binding protein }\end{array}$ & $\begin{array}{ll}- & \text { scavenger receptor activity } \\
- & \text { isomerase activity } \\
- & \text { cellular defense response } \\
\text { - } & \text { signal transduction }\end{array}$ & 1.66 & 1.19E-05 \\
\hline MGI:1921298 & $\begin{array}{l}4632428 \mathrm{NO} \\
5 \mathrm{Rik}\end{array}$ & $\begin{array}{l}\text { RIKEN cDNA 4632428N05 } \\
\text { gene }\end{array}$ & - $\quad$ receptor activity & 1.63 & $1.48 \mathrm{E}-06$ \\
\hline MGI:96614 & $\operatorname{ltg} b 5$ & integrin beta 5 & $\begin{array}{ll}- & \text { integrin binding } \\
- & \text { receptor activity } \\
\text { - } & \text { cell adhesion }\end{array}$ & 1.56 & $1.05 \mathrm{E}-06$ \\
\hline MGI:1914877 & Olfm/3 & olfactomedin-like 3 & - $\quad$ scaffold protein & 1.53 & 1.36E-04 \\
\hline MGI:96073 & Hexa & hexosaminidase $\mathrm{A}$ & $\begin{array}{l}\text { beta- } \mathrm{N}- \\
\text { acetylhexosaminidase } \\
\text { activity } \\
\text { - } \quad \text { protein heterodimerization } \\
\text { activity } \\
\text { - } \quad \text { hydrolase activity }\end{array}$ & 1.51 & $3.85 \mathrm{E}-06$ \\
\hline MGl:1339758 & Csf1r & $\begin{array}{l}\text { colony stimulating factor } 1 \\
\text { receptor }\end{array}$ & $\begin{array}{l}\text { - } \quad \text { macrophage colony- } \\
\text { stimulating factor receptor } \\
\text { activity } \\
\text { - } \quad \text { protein homodimerization } \\
\text { activity } \\
\text { - } \quad \text { ATP binding } \\
\text { immune response }\end{array}$ & 1.45 & $1.58 \mathrm{E}-06$ \\
\hline MGI:107387 & Aqp4 & aquaporin 4 & $\begin{array}{ll}\text { - } & \text { porin activity } \\
\text { - } & \text { water transmembrane } \\
\text { transporter activity }\end{array}$ & 1.42 & $8.90 \mathrm{E}-07$ \\
\hline MGI:1918089 & P2ry12 & $\begin{array}{l}\text { purinergic receptor P2Y. } \\
\text { G-protein coupled } 12\end{array}$ & $\begin{array}{ll}\text { - } & \text { ADP receptor activity } \\
\text { - } & \text { guanyl-nucleotide } \\
\text { exchange factor activity } \\
\text { - } & \text { G-protein coupled } \\
\text { - } & \text { adenosine receptor activity } \\
\text { signal transducer activity }\end{array}$ & 1.40 & $2.68 \mathrm{E}-04$ \\
\hline MGI:1278340 & Rpl21 & ribosomal protein L21 & $\begin{array}{l}\text { - } \quad \text { structural constituent of } \\
\text { ribosome } \\
\text { - } \quad \text { RNA binding }\end{array}$ & 1.37 & $2.20 \mathrm{E}-04$ \\
\hline MGI:107286 & Man2b1 & mannosidase 2. alpha B1 & $\begin{array}{ll}\text { - } & \text { carbohydrate binding } \\
\text { - } & \text { alpha-mannosidase activity } \\
\text { - } & \text { hydrolase activity }\end{array}$ & 1.35 & 4.77E-05 \\
\hline MGI:1096881 & Eef1a1 & $\begin{array}{l}\text { eukaryotic translation } \\
\text { elongation factor } 1 \text { alpha } 1\end{array}$ & $\begin{array}{ll}- & \text { GTPase activity } \\
- & \text { translation elongation } \\
& \text { factor activity } \\
\text { - } & \text { regulation of transcription }\end{array}$ & 1.32 & $6.22 \mathrm{E}-06$ \\
\hline MGI:1915213 & Npc2 & Niemann Pick type C2 & $\begin{array}{ll}\text { - } & \text { cholesterol binding } \\
\text { - } & \text { enzyme binding }\end{array}$ & 1.27 & $1.20 \mathrm{E}-03$ \\
\hline MGI:88561 & Ctsb & cathepsin B & $\begin{array}{ll}\text { - } & \text { cysteine-type peptidase } \\
\text { - } & \text { activity } \\
\text { - } & \text { imydrolase activity } \\
\text { immune response }\end{array}$ & 1.25 & $1.21 \mathrm{E}-05$ \\
\hline MGI:88564 & Ctsl & cathepsin L & $\begin{array}{ll}\text { - } & \text { cysteine-type peptidase } \\
\text { - } & \text { hydivity } \\
\text { - } & \text { histone binding } \\
\text { - } & \text { immune response }\end{array}$ & 1.23 & $3.42 \mathrm{E}-04$ \\
\hline
\end{tabular}




\begin{tabular}{|c|c|c|c|c|c|}
\hline MGl:1934664 & Rpph1 & $\begin{array}{l}\text { ribonuclease P RNA } \\
\text { component } \mathrm{H} 1\end{array}$ & - $\quad$ endoribonuclease activity & 1.23 & $1.21 \mathrm{E}-05$ \\
\hline MGI:1920174 & Anln & anillin & $\begin{array}{l}\text { - } \quad \text { actin binding } \\
\text { - } \quad \text { phospholipid binding }\end{array}$ & 1.22 & $1.53 \mathrm{E}-03$ \\
\hline MGl:95832 & Grn & granulin & $\begin{array}{ll}\text { - } & \text { growth factor activity } \\
\text { - } & \text { cytokine activity } \\
\text { - } & \text { signal transduction }\end{array}$ & 1.21 & $1.00 \mathrm{E}-03$ \\
\hline MGI:98729 & Tgfbr2 & $\begin{array}{l}\text { transforming growth factor. } \\
\text { beta receptor II }\end{array}$ & $\begin{array}{ll}\text { - } & \text { ATP binding } \\
\text { - } & \text { transmembrane receptor } \\
& \text { protein serine/threonine } \\
& \text { kinase activity } \\
\text { - } & \text { transferase activity } \\
\text { - } & \text { receptor activity } \\
\text { - } & \text { SMAD binding } \\
\text { - } & \text { signal transduction }\end{array}$ & 1.18 & 4.94E-03 \\
\hline MGI:894320 & $\operatorname{Prd} \times 6$ & peroxiredoxin 6 & $\begin{array}{ll}\text { - } & \text { glutathione peroxidase } \\
\text { - } & \text { octivity } \\
\text { - } & \text { antioreductase activity } \\
\text { - } & \text { response to oxidative } \\
\text { stress }\end{array}$ & 1.17 & 2.21E-04 \\
\hline MGI:1921305 & Plce1 & phospholipase C. epsilon 1 & $\begin{array}{ll}\text { - } & \text { guanyl-nucleotide } \\
\text { - } & \text { calchange factor activity } \\
\text { - } & \text { receptor signaling protein } \\
& \text { activity } \\
\text { - } & \text { hydrolase activity } \\
\text { - } & \text { signal transducer activity }\end{array}$ & 1.15 & $5.42 E-03$ \\
\hline MGl:107357 & Inpp5d & $\begin{array}{l}\text { inositol polyphosphate-5- } \\
\text { phosphatase D }\end{array}$ & $\begin{array}{ll}- & \text { SH3 domain binding } \\
\text { - } & \text { PTB domain binding } \\
\text { - } & \text { hydrolase activity } \\
\text { - } & \text { signal transducer activity } \\
\text { - } & \text { immune Response }\end{array}$ & 1.14 & 1.96E-02 \\
\hline MGI:1330838 & Lgmn & legumain & $\begin{array}{ll}\text { - } & \text { cysteine-type } \\
& \text { endopeptidase activity } \\
\text { - } & \text { peptidase activity } \\
\text { - } & \text { immune Response } \\
\text { - } & \text { hydrolase activity }\end{array}$ & 1.07 & $1.82 \mathrm{E}-03$ \\
\hline MGI:1917329 & Golm1 & golgi membrane protein 1 & $\begin{array}{ll}\text { - } & \text { protein modification } \\
\text { - } & \text { nucleus organization }\end{array}$ & 1.03 & $2.85 \mathrm{E}-02$ \\
\hline MGI:88385 & Cfh & $\begin{array}{l}\text { complement component } \\
\text { factor } \mathrm{h}\end{array}$ & $\begin{array}{ll}\text { - } & \text { heparin binding } \\
\text { - } & \text { heparan sulfate } \\
\text { proteoglycan binding } \\
\text { - } \quad \text { complement activation } \\
\text { immune response }\end{array}$ & 1.02 & 4.65E-02 \\
\hline MGI:95640 & Gapdh & $\begin{array}{l}\text { glyceraldehyde-3-phosphate } \\
\text { dehydrogenase }\end{array}$ & $\begin{array}{ll}-\quad & \text { microtubule binding } \\
-\quad & \text { NADP Binding }\end{array}$ & 1.01 & 3.07E-03 \\
\hline MGI:1924096 & Rps9 & ribosomal protein S9 & $\begin{array}{ll}\text { - } & \text { structural constituent of } \\
\text { - } & \text { ribosome } \\
\text { - } & \text { RNA binding } \\
\text { translation regulator activity }\end{array}$ & 1.01 & $1.59 \mathrm{E}-02$ \\
\hline MGI:97171 & Mt1 & metallothionein 1 & $\begin{array}{l}\text { organic cyclic compound } \\
\text { binding } \\
\text { - } \quad \text { hormone binding } \\
\text { - } \quad \text { copper ion binding }\end{array}$ & 1.01 & $3.08 \mathrm{E}-03$ \\
\hline
\end{tabular}




\begin{tabular}{|c|c|c|c|c|c|}
\hline MGl:97591 & $P k m$ & pyruvate kinase. muscle & $\begin{array}{ll}\text { - } & \text { magnesium ion binding } \\
\text { - } & \text { pyruvate kinase activity } \\
& \text { ATP Binding }\end{array}$ & 1.00 & $1.56 \mathrm{E}-03$ \\
\hline MGl:88423 & Clu & clusterin & $\begin{array}{ll}\text { - } & \text { ATPase activity } \\
\text { - } & \text { ubiquitin protein ligase } \\
\text { binding } \\
\text { - } \quad \text { misfolded protein binding } \\
\text { - } \quad \text { immune response }\end{array}$ & 0.96 & $2.50 \mathrm{E}-03$ \\
\hline MGI:1338892 & Padi2 & $\begin{array}{l}\text { peptidyl arginine deiminase. } \\
\text { type II }\end{array}$ & $\begin{array}{ll}\text { - } & \text { protein-arginine deiminase } \\
& \text { activity } \\
\text { - } & \text { calcium ion binding } \\
\text { - } & \text { hydrolase activity } \\
\text { - } & \text { immune response }\end{array}$ & 0.95 & $4.86 \mathrm{E}-02$ \\
\hline MGI:1915472 & Tubb4b & tubulin. beta 4B class IVB & $\begin{array}{ll}\text { - } & \text { structural molecule activity } \\
\text { - } & \text { GTPase Activity } \\
\text { double-stranded RNA } \\
\text { binding } \\
\text { - } & \begin{array}{l}\text { structural constituent of } \\
\text { cytoskeleton }\end{array} \\
\text { - } & \text { unfolded protein binding }\end{array}$ & 0.93 & 2.03E-02 \\
\hline MGl:2445114 & Pisd & $\begin{array}{l}\text { phosphatidylserine } \\
\text { decarboxylase }\end{array}$ & - $\quad$ lyase activity & 0.93 & $1.94 \mathrm{E}-02$ \\
\hline MGI:96247 & Hsp90ab1 & $\begin{array}{l}\text { heat shock protein } 90 \text { alpha } \\
\text { (cytosolic). class B member } 1\end{array}$ & $\begin{array}{ll}\text { - } & \text { unfolded protein binding } \\
\text { - } & \text { GTP binding } \\
\text { - } & \text { ATP binding } \\
\text { - } & \text { double-stranded RNA } \\
\text { - } & \text { binding } \\
\text { - } & \text { immune response } \\
\text { - } & \text { negative regulation of } \\
& \text { neuron apoptotic process }\end{array}$ & 0.90 & $6.13 E-03$ \\
\hline MGl:1925017 & Ermn & ermin. ERM-like protein & actin filament binding & 0.90 & $3.02 \mathrm{E}-02$ \\
\hline MGI:105959 & Cox8a & $\begin{array}{l}\text { cytochrome c oxidase subunit } \\
\text { VIIla }\end{array}$ & $\begin{array}{l}\text { - cytochrome-c oxidase } \\
\text { activity }\end{array}$ & 0.88 & $1.52 \mathrm{E}-02$ \\
\hline MGl:96748 & Lamp2 & $\begin{array}{l}\text { Iysosomal-associated } \\
\text { membrane protein } 2\end{array}$ & $\begin{array}{ll}\text { - } & \text { membrane glycoprotein } \\
\text { - } & \text { TRNA ligase activity } \\
\text { - } & \text { ATP binding } \\
\text { hemostasis }\end{array}$ & 0.88 & $2.94 \mathrm{E}-02$ \\
\hline MGI:97748 & Ctsa & cathepsin A & $\begin{array}{ll}\text { - } & \text { enzyme activator activity } \\
\text { - } & \text { cerine-type } \\
\text { - } & \text { hydrolase activity }\end{array}$ & 0.86 & 3.15E-02 \\
\hline MGI:103099 & Cox6a1 & $\begin{array}{l}\text { cytochrome c oxidase subunit } \\
\text { Vla polypeptide } 1\end{array}$ & $\begin{array}{l}\text { - cytochrome-c oxidase } \\
\text { activity }\end{array}$ & 0.85 & $2.02 \mathrm{E}-02$ \\
\hline MGI:1346074 & Fxr2 & $\begin{array}{l}\text { fragile } X \text { mental retardation. } \\
\text { autosomal homolog } 2\end{array}$ & $\begin{array}{ll}\text { - } & \text { RNA binding } \\
\text { - } & \text { identical protein binding }\end{array}$ & 0.84 & $3.11 \mathrm{E}-02$ \\
\hline MGI:99607 & Abca1 & $\begin{array}{l}\text { ATP-binding cassette. sub- } \\
\text { family } A(A B C 1) \text {. member } 1\end{array}$ & $\begin{array}{ll}\text { - } & \text { apolipoprotein binding } \\
\text { - } & \text { phospholipid binding } \\
\text { - } & \text { cholesterol binding } \\
\text { - } & \text { ATP binding }\end{array}$ & 0.82 & 4.37E-02 \\
\hline MGI:88252 & Calr & calreticulin & $\begin{array}{ll}\text { - } & \text { iron ion binding } \\
\text { - } & \text { calcium ion binding } \\
\text { - } & \text { hormone binding } \\
\text { - } & \text { mRNA binding } \\
\text { - } & \text { regulation of transcription } \\
\text { - } & \text { signal transduction } \\
\text { - } & \text { immune system }\end{array}$ & 0.81 & $3.02 E-02$ \\
\hline
\end{tabular}




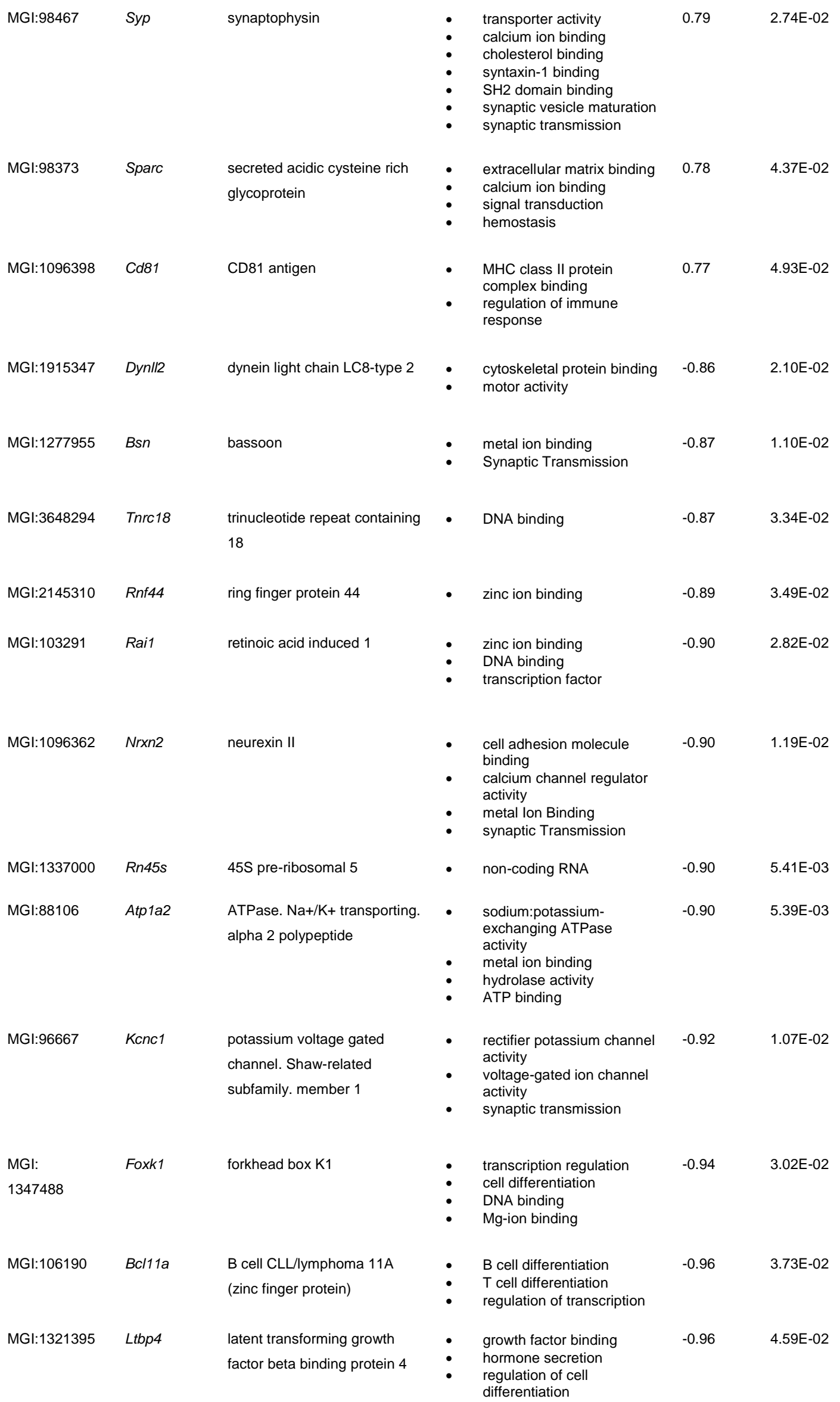




\begin{tabular}{|c|c|c|c|c|c|}
\hline MGl:2176606 & Scrt1 & $\begin{array}{l}\text { scratch homolog 1. zinc finger } \\
\text { protein }\end{array}$ & - $\quad$ transcription regulation & -0.96 & 2.09E-02 \\
\hline MGI:1925589 & Ttyh3 & tweety homolog 3 & $\begin{array}{ll}\text { - } & \text { chloride channel activity } \\
\text { - } & \text { transmembrane transport }\end{array}$ & -0.96 & 7.91E-03 \\
\hline MGI:2444218 & Ahdc1 & $\begin{array}{l}\text { AT hook. DNA binding motif. } \\
\text { containing } 1\end{array}$ & - $\quad$ DNA binding & -0.97 & $1.84 \mathrm{E}-02$ \\
\hline MGI:98460 & Syn1 & synapsin I & $\begin{array}{l}\text { - } \quad \text { Neurotransmitter secretion } \\
\text { - } \quad \text { Synaptic vesicle transport }\end{array}$ & -0.98 & $3.98 \mathrm{E}-03$ \\
\hline MGI:95617 & Gabra5 & $\begin{array}{l}\text { gamma-aminobutyric acid } \\
\text { (GABA) A receptor. subunit } \\
\text { alpha } 5\end{array}$ & $\begin{array}{ll}- & \text { GABA- receptor } \\
- & \text { chloride transport } \\
- & \text { gamma-aminobutyric acid } \\
& \text { signaling pathway }\end{array}$ & -0.99 & $1.52 \mathrm{E}-02$ \\
\hline MGI:2143099 & Al593442 & $\begin{array}{l}\text { expressed sequence } \\
\text { Al593442 }\end{array}$ & - $\quad$ unclassified & -1.00 & 4.30E-03 \\
\hline MGI:1346031 & Tshz1 & $\begin{array}{l}\text { teashirt zinc finger family } \\
\text { member } 1\end{array}$ & $\begin{array}{l}\text { - } \quad \text { transcription factor } \\
\text { DNA binding }\end{array}$ & -1.00 & 1.73E-02 \\
\hline MGI:2444817 & $\begin{array}{l}\text { C530008M1 } \\
\text { 7Rik }\end{array}$ & $\begin{array}{l}\text { RIKEN cDNA C530008M17 } \\
\text { gene }\end{array}$ & - unknown & -1.00 & 2.26E-02 \\
\hline MGI:2441680 & Tmem8b & transmembrane protein $8 \mathrm{~B}$ & $\begin{array}{l}\text { - } \quad \text { cell cycle regulation } \\
\text { - } \quad \text { cell matrix adhesion }\end{array}$ & -1.00 & 2.27E-02 \\
\hline MGI:109169 & Epas1 & $\begin{array}{l}\text { endothelial PAS domain } \\
\text { protein } 1\end{array}$ & $\begin{array}{ll}\text { - } & \text { regulation of transcription } \\
\text { - } & \text { angiogenesis } \\
\text { - } & \text { transcription } \\
\text { - } & \text { signal transduction } \\
\text { cellular stress response }\end{array}$ & -1.01 & $6.32 \mathrm{E}-03$ \\
\hline MGI:1351323 & Snord33 & $\begin{array}{l}\text { small nucleolar RNA. C/D box } \\
33\end{array}$ & - unknown & -1.04 & 4.35E-02 \\
\hline MGI:107363 & Stxbp1 & syntaxin binding protein 1 & $\begin{array}{ll}\text { - } & \text { release of } \\
& \text { neurotransmitters via } \\
\text { syntaxin regulation } \\
\text { - } \quad & \text { vesicle transport } \\
\text { - } \quad \text { exocytosis } \\
\text { - } \quad \text { regulation of insulin } \\
\text { secretion }\end{array}$ & -1.04 & 7.75E-04 \\
\hline MGI:2443847 & Sdk2 & sidekick homolog 2 & $\begin{array}{ll}\text { - } & \text { chemotaxis } \\
\text { - } & \text { protein targeting } \\
\text { - } & \text { cell adhesion }\end{array}$ & -1.04 & $2.43 \mathrm{E}-02$ \\
\hline MGI:1919559 & Tmem158 & transmembrane protein 158 & Ras pathway & -1.06 & 2.27E-02 \\
\hline MGI:102858 & Fosl2 & fos-like antigen 2 & $\begin{array}{ll}\text { - } & \text { regulation of transcription } \\
\text { - } & \text { cell regulation } \\
\text { - } & \text { fibroblasten proliferation }\end{array}$ & -1.10 & $1.07 \mathrm{E}-02$ \\
\hline MGI:2686934 & Zfhx2 & zinc finger homeobox 2 & $\begin{array}{l}\text { - } \quad \text { DNA binding } \\
\text { transcriptional factor } \\
\text { activity }\end{array}$ & -1.11 & $6.32 \mathrm{E}-03$ \\
\hline
\end{tabular}




\begin{tabular}{|c|c|c|c|c|c|}
\hline MGI:96434 & $\lg f 2$ & insulin-like growth factor 2 & $\begin{array}{ll}\text { - } & \text { hormone activity } \\
\text { - } & \text { growth factor activity } \\
\text { - } & \text { cell proliferation } \\
\text { - } & \text { regulation of cell cycle } \\
\text { - } & \text { hetein Metabolism } \\
\text { - } & \text { signal transduction }\end{array}$ & -1.11 & 4.47E-03 \\
\hline MGl:2444034 & $\begin{array}{l}9530091 C 0 \\
8 R i k\end{array}$ & $\begin{array}{l}\text { RIKEN cDNA 9530091C08 } \\
\text { gene }\end{array}$ & $\begin{array}{l}\text { - unclassified non-coding } \\
\text { RNA gene }\end{array}$ & -1.14 & 1.10E-02 \\
\hline MGI:1313277 & Vamp2 & $\begin{array}{l}\text { vesicle-associated membrane } \\
\text { protein } 2\end{array}$ & $\begin{array}{l}\text { - } \quad \text { vesicle mediate transport } \\
\text { - } \quad \text { synaptic vesicle exocytosis } \\
\quad \text { regulation of insulin } \\
\text { secretion }\end{array}$ & -1.14 & $2.79 \mathrm{E}-04$ \\
\hline MGI:1890616 & Scube1 & $\begin{array}{l}\text { signal peptide. CUB domain. } \\
\text { EGF-like } 1\end{array}$ & $\begin{array}{ll}- & \text { inflammatory response } \\
\text { - } & \text { endothelial cell } \\
& \text { differentiation }\end{array}$ & -1.19 & $1.28 \mathrm{E}-03$ \\
\hline MGI:2444210 & Nr1d1 & $\begin{array}{l}\text { nuclear receptor subfamily } 1 . \\
\text { group D. member } 1\end{array}$ & $\begin{array}{ll}\text { - } & \text { transcription factor } \\
\text { - } & \text { insulin secretion } \\
\text { - } & \text { metabolic processes } \\
\text { - } & \text { inflammatory processes }\end{array}$ & -1.23 & $6.34 \mathrm{E}-04$ \\
\hline MGI:2444521 & Rnf165 & ring finger protein 165 & - $\quad$ zinc ion binding & -1.25 & $1.05 \mathrm{E}-02$ \\
\hline MGl:1351339 & Grm2 & $\begin{array}{l}\text { glutamate receptor. } \\
\text { metabotropic } 2\end{array}$ & $\begin{array}{ll}-\quad & \text { synaptic transmission } \\
\text { - } \quad & \text { glutamate secretion }\end{array}$ & -1.27 & 2.12E-03 \\
\hline MGI:102703 & Gng4 & $\begin{array}{l}\text { guanine nucleotide binding } \\
\text { protein (G protein). gamma } 4\end{array}$ & $\begin{array}{ll}- & \text { signal transduction } \\
- & \text { GTPase activity } \\
\text { - } & \text { hemostasis } \\
\text { - } & \text { synaptic transmission } \\
\text { - } & \text { glucagon response } \\
\text { - } & \text { transmembrane transport } \\
& \text { of small molecules }\end{array}$ & -1.42 & 7.64E-05 \\
\hline $\begin{array}{l}\text { MGl: } \\
95295\end{array}$ & Egr1 & early growth response 1 & $\begin{array}{ll}\text { - } & \text { transcriptional regulator } \\
\text { - } & \text { immune response } \\
\text { - } & \text { T cell differentiation }\end{array}$ & -1.55 & $6.15 \mathrm{E}-07$ \\
\hline
\end{tabular}




\section{$4 \quad$ DISCUSSION}

\subsection{Project 1: Characterization of the Tg4-42 mouse model}

$A \beta_{4-42}$ is one of the major $A \beta$ isoforms in the $A D$ brain. However, little is known about its contribution to the etiology of AD. The main objective of the first part of the thesis was to investigate the consequences of $A \beta_{4-42}$ in vivo by examining Tg4-42 mice expressing $A \beta_{4-42 .}$.

Next to $A \beta$ starting with aspartate as the first amino acid, a variety of modified and $N$-truncated $A \beta$ variants have been characterized (Kummer and Heneka, 2014). $\mathrm{N}$-truncated $A \beta$ can be found plaque-associated as well as intraneuronal (Gouras et al., 2000). $A \beta_{4-42}$ has first been discovered in 1985 by Masters et al. They demonstrated that $64 \%$ of $A \beta$ peptides started with a Phe- 4 residue in plaque cores of two sporadic $A D$ cases (Masters et al., 1985). Later it could be shown that $A \beta_{4-42}$ is relatively prevalent in $A D$, vascular dementia patients and aged controls (Lewis et al., 2006). While the exact amount of $A \beta_{4-42}$ is not clear, a recent study, using immunoprecipitation and mass spectroscopy, could show that $A \beta_{4-42}$ is one of the major fractions in the cortex and hippocampus of $A D$ patients (Portelius et al., 2010). Moreover, $A \beta_{4-42}$ has also been identified in amyloid deposits from familial Danish dementia and vascular dementia patients (Tomidokoro et al., 2005; Lewis et al., 2006). To date, it is not fully understood whether $\mathrm{N}$-truncated $A \beta_{4-x}$ is derived from full-length $A \beta_{1-42}$ or directly from APP. It is proposed that the degrading enzyme neprilysin is responsible for the cleavage of $A \beta_{1-42}$ and the generation of $A \beta_{4-42}$ (Howell et al., 1995; Iwata et al., 2001; Carson and Turner, 2002).

Pike et al. (1995b) showed that N-terminal deletions enhance aggregation and toxicity of $A \beta$. Peptides with $N$-terminal deletions including $A \beta_{4-42}$ showed in comparison to full-length $A \beta$ enhanced peptide aggregation into neurotoxic, $\beta$-sheet fibrils. Moreover, is has been demonstrated that the $N$-terminus specifies the fibrillization behavior (Haupt et al., 2012). In addition, it has also been reported that pyroglutamate $A \beta$ is more toxic than full-length $A \beta$ (Russo et al., 1997). Here it could be shown that freshly prepared 
$N$-truncated $A \beta$ variants $A \beta_{4-38}, A \beta_{4-40}, A \beta_{4-42}$ and $A \beta_{P E 3-42}$ rapidly form stable aggregates similar to $A \beta_{1-42}$ under reducing conditions. All peptides displayed dimeric oligomers and monomers, while $A \beta_{1-42}, A \beta_{p E 3-42}$ and $A \beta_{4-42}$ also developed SDS-stable tri- or tetrameric oligomers. Moreover, aged $A \beta_{1-42}, A \beta_{p E 3-42}$ and $A \beta_{4-42}$ peptides retained this pattern and exhibited in addition higher molecular weight aggregates. In line with these observations, we could recently show that $A \beta_{4-42}, A \beta_{p E 3-42}$ and $A \beta_{1-42}$ are unstructured in the monomeric state and have a high propensity to form folded structures upon heating. Using NMR spectroscopy, far-UV CD spectroscopy and dynamic light scattering, we demonstrated that $A \beta_{4-42}$ and $A \beta_{p E 3-42}$ have a higher tendency to form stable aggregates than $A \beta_{1-42}$ (Bouter et al., 2013). The observation that $A \beta_{4-42}$ and $A \beta_{p E 3-42}$ have a more pronounced tendency to form aggregates than the $N$-terminally intact $A \beta_{1-42}$ indicate that $A \beta_{4-42}$ and $A \beta_{P E 3-42}$ aggregation may precede $A \beta_{1-42}$ aggregation in vivo.

There is increasing evidence, that $N$-truncated $A \beta$ variants, like $A \beta_{4-42}$, may contribute to the AD pathology (Jawhar et al., 2011; Bouter et al., 2013). The removal of the first three amino acids increases $A \beta$ hydrophobicity of the $N$-terminal $A \beta$ part and may influence its toxicity. We could lately demonstrate through short-term treatment of primary cortical neurons, that $A \beta_{4-42}$ is as toxic as $A \beta_{p E 3-42}$ and $A \beta_{1-42}$. Furthermore, treatment of wild-type mice using intraventricular $A \beta$ injection with $A \beta_{4-42}$ induced significant working memory deficits. These deficits were similar to the ones induced through $A \beta_{p E 3-42}$ and $A \beta_{1-42}$ (Bouter et al., 2013).

\subsection{1}

\section{$A \beta_{4-x}$ precedes $A \beta_{p E 3-x}$ accumulation in 5XFAD mice}

Next to human $A D$ patients $N$-terminal truncated and modified $A \beta$ variants have also been observed in several $A D$ mouse models. In $A P P / P S 1 K I$ mice a variety of $N$-truncated $A \beta$ peptides have been described. Applying two dimensional gel electrophoresis in combination with mass spectroscopy a complex pattern of $\mathrm{N}$-truncated variants, including $A \beta_{4 / 5-42}, A \beta_{8 / 9 / 10 / 11-42}$ and $A \beta_{2 / 3-42}$, were detected starting at 2.5 months of age. Moreover, pyroGlu-modified $A \beta_{3-x}$ could be shown at 6 months and increased with age (Casas et al., 2004). Pyroglutamate $A \beta$ peptides are a highly abundant $N$-truncated $A \beta$ species in the brain of $A D$ patients and have been detected in several other $A D$ mouse models next to APP/PS1KI (Hartig et al., 2010; Jawhar et al., 2011; Frost et al., 2013). Harigaya et al. (2000) reported that $25 \%$ of the total $A \beta_{x-42}$ in $A D$ plaques consisted of $A \beta_{p E 3-42}$.

As stated before, $N$-truncated $A \beta$ variants show a higher toxicity compared to fulllength $A \beta$. To further analyze the in vivo toxicity of pyroglutamate $A \beta$ several mouse models over-expressing $A \beta_{3-42}$ were generated. TBA2.1 is an example for a mouse model expressing $A \beta_{3-42}$ with glutamine rather than the glutamate normally found at the third 
position of $A \beta$. The alteration promotes the spontaneous and enzymatic catalyzed formation of $A \beta_{p E 3-42 .}$ TBA2.1 mice exhibit abundant intracellular $A \beta_{p E 3-42}$ and neuron loss in $A \beta_{p E 3-42}$ accumulating neurons (Wirths et al., 2009; Alexandru et al., 2011). Using mass spectrometric analysis, high amounts of $A \beta_{p E 3-42}$ were detected in the widely used 5XFAD mouse model (Wittnam et al., 2012). Similar to APP/PS1KI mice, 5XFAD animals displayed a highly heterogeneous constitution of $\mathrm{N}$-truncated and modified $A \beta$ peptides. Interestingly, both transgenic lines develop a significant brain-area specific neuron loss (Casas et al., 2004; Oakley et al., 2006; Breyhan et al., 2009; Christensen et al., 2010; Jawhar et al., 2010; Bouter et al., 2013). The pathological observations in 5XFAD and APP/PS1KI mice might be at least partly triggered by truncated and modified $A \beta$ including $A \beta_{4-42}$. The following $A \beta$ variants were detected in the brain of 5 XFAD mice: $A \beta_{1-42}, A \beta_{1-40}$, $A \beta_{p E 3-40}, A \beta_{p E 3-42}, A \beta_{3-42}, A \beta_{4-42}$ and $A \beta_{5-42}$. Strikingly, $A \beta_{4-42}$ was the most abundant $\mathrm{N}$-truncated $A \beta$ form in 5XFAD mice (Wittnam et al., 2012). The newly generated antibody NT4X-167 recognizes the $N$-terminus of $N$-truncated $A \beta$ with a preference for $A \beta_{4-42}$. Therefore it allows to differentiate between the two major $N$-truncated $A \beta$ variants, $A \beta_{P E 3-42}$ and $A \beta_{4-42}$ (Antonios et al., 2013). Here, NT4X-167 was used in 5XFAD mice in combination with IC16 that is specific for $A \beta_{1-x}$ and 1-57, an antibody that exclusively reacts with $A \beta_{p E 3-x}$. It could be shown that $A \beta_{4-x}$ preceded $A \beta_{p E 3-x}$ accumulation in the brain of young $5 X F A D$ mice. More importantly, $A \beta_{4-x}$ could be detected together with $A \beta_{1-x}$ intraneuronal in cortical neurons of 6 weeks old homozygous 5XFAD mice. Previously, it has been demonstrated that cortical neurons are prone to degeneration in aged 5XFAD mice (Jawhar et al., 2010; Eimer and Vassar, 2013). Transient and early intraneuronal accumulation of $A \beta$ also correlates with neuron loss in a variety of other APP/A $\beta$ mouse models (Casas et al., 2004; Christensen et al., 2008; Christensen et al., 2010; Alexandru et al., 2011). As expected, NT4X-167 also detected abundant $A \beta_{4-x}$ accumulation in amyloid plaques of 12-month-old 5XFAD mice. The results in 5XFAD mice using the NT4X-167 antibody emphasizes the important role of $A \beta_{4-x}$ in the pathology of $A D$.

\subsection{2}

\section{Intraneuronal $A \beta$ expression in Tg4-42 mice}

In order to study the long-lasting neurotoxic effects of $A \beta_{4-42}$ the transgenic mouse model Tg4-42 was generated (Bouter et al., 2012). This model is the first transgenic mouse model expressing exclusively $\mathrm{N}$-truncated $A \beta_{4-42}$. Tg4-42 mice express human $N$-terminally truncated $A \beta_{42}$ in which the first three amino acids have been removed. $A$ murine TRH signal peptide is fused to the $A \beta N$-terminus and routes $A \beta_{4-42}$ through the secretory pathway. After release of the TRH signal peptide, $A \beta_{4-42}$ is liberated into the trans-Golgi and secretory granules. The $A \beta_{4-42}$ expression in Tg4-42 mice happens in a 
region-specific manner consistent with the transgene expression driven by the murine Thy1 promoter (Caroni, 1997). Intracellular A $\beta$ was predominantly expressed in the CA1 of the hippocampus beginning at two months of age. $A \beta_{4-42}$ expression in the CA1 pyramidal cell layer of the hippocampus declined during aging in Tg4-42 and Tg4-42 hom mice. The decrease of $A \beta$ expression can be attributed to the extensive neuron loss in this brain region. Intraneuronal $A \beta$ was also observed in Tg4-42 mice in the occipital cortex, piriform cortex, striatum and superior colliculus starting at two months of age.

Next to Tg4-42 mice several other transgenic mouse models have been generated expressing major $A \beta$ forms without over-expressing mutant APP or PSEN. The direct expression of $A \beta_{42}$ in the APP48 mouse model and the $G 2$ model led to specific intracellular $A \beta_{42}$ expression in the brain. In both models intracellular toxicity of $A \beta_{42}$ could be demonstrated, while extracellular $A \beta$ deposits were mostly lacking (LaFerla et al., 1995; Abramowski et al., 2012). Similar to these models, Tg4-42 mice showed intracellular $A \beta$ accumulation in the absence of extracellular deposits.

Growing evidence suggests that intraneuronal $A \beta$ accumulation contributes to the pathological events in $A D$. Intraneuronal $A \beta$ accumulation has also been detected in a variety of AD mouse models including Tg2576, 3xTg, APP/PS1KI and 5XFAD (Takahashi et al., 2002; Oddo et al., 2003; Oakley et al., 2006). Grundke-lqbal et al. (1989) reported already 25 years ago that intracellular $A \beta$ precedes $A \beta$ plaque formation in $A D$ patients. In addition, it has been shown that intracellular $A \beta$ also appears before NFT (Gouras et al., 2000; Fernandez-Vizarra et al., 2004). Later, it could be demonstrated that mainly longer $A \beta_{42}$ variants accumulate within neurons in $A D$ vulnerable regions. CA1 pyramidal neurons of sporadic and familial $A D$ patients showed significantly increased $A \beta_{42}$ levels compared to healthy individuals (Aoki et al., 2008). With increasing plaque pathology and cognitive impairments, the amount of $A \beta$ within neurons decreased. It is hypothesized that intraneuronal $A \beta$ causes early neuronal impairments (Gouras et al., 2000). Furthermore, it has been suggested that intraneuronal accumulation of $A \beta_{42}$ might lead to cell lysis and thus contributing to increased extracellular amyloid deposits and neuron loss (D'Andrea et al., 2001; Wirths and Bayer, 2012).

\subsection{3}

\section{Neuron loss in Tg4-42 mice}

Neuron loss and atrophy are one of the major neuropathological hallmarks in AD patients. However, many transgenic $A D$ mouse models lack this cardinal feature of $A D$. Most mouse models that do show a substantial neuron loss express multiple APP and PSEN-1 mutations. For example, 5XFAD mice display a significant neuron loss in the fifth cortical layer of the frontal cortex beginning at 9 months. Using unbiased stereology it could be 
demonstrate that 5XFAD mice develop a significant selective neuron loss in layer 5 of the cortex, while the overall neuron number of the total frontal cortex and hippocampus remained unaltered. Strikingly, these observations are in line with the accumulation of intraneuronal $A \beta$ in the cortical layer 5, but not in the CA1 (Jawhar et al., 2010; Eimer and Vassar, 2013). Similar to 5XFAD mice, a massive neuron loss was observed in APP/PS1K1 mice. Neuron loss was found in the frontal cortex and in the CA1 of the hippocampus, both regions with extensive $A \beta$ intraneuronal accumulation (Christensen et al., 2008; Breyhan et al., 2009). Strikingly, in both mouse models, despite the appearance of plaques, neuron loss occurred only in regions with intraneuronal $A \beta$. These observations argue against the role of extracellular plaques in terms of $A \beta$ toxicity.

A recently developed mouse model expressing human APP harboring the E693 $\triangle$ mutation show neuron loss in the CA3 of the hippocampus. Intraneuronal A $\beta$ oligomer accumulation preceded the neuron loss in these mice, while mice showed no extracellular plaques (Tomiyama et al., 2010). In line with these findings, long-term exposure to $\mathrm{N}$-truncated $A \beta_{4-42}$ induced an age- and dose-dependent neuron loss in hemizygous and homozygous Tg4-42 mice in the CA1 layer of the hippocampus. Unbiased stereology revealed a $38 \%$ neuron loss in hemizygous Tg4-42 mice and a $65 \%$ neuron loss in Tg4-42 $2_{\text {hom }}$ at eight months. Furthermore, 12-month-old Tg4-42 mice showed a $51 \%$ decrease in neuron number in the CA1 compared to same-aged WT. To expand these findings and determine in greater detail the degree to which $\mathrm{Tg} 4-42$ mirror the progressive neuron loss observed in human $A D$, neurons were additionally counted at ages two, four, five and six months of age. Compared to 2-month-old Tg4-42 hom mice 3-month-old mice already showed a decreased number of neurons, although this decrease was not significant. However, this trend suggests early neurodegeneration, even at a time when statistically significant neuron loss is not yet evident. Furthermore, the detailed stereological characterization of $\mathrm{Tg} 4-42_{\text {hom }}$ mice revealed a progressive neuron loss starting with four months of age. The hippocampus belongs to the brain areas that are known to be affected in $A D$ and contributes to the learning and memory deficits observed in $A D$ patients. Some of the earliest damages in $A D$ brains have been reported in the hippocampus. AD patients display significant neuronal loss in the hippocampus. Padurariu et al. (2012) reported a decrease in neuronal density especially in the CA1 and CA3 regions of the hippocampus, although the effects in the CA1 area were even more severe.

The progressive loss of neurons in Tg4-42 and Tg4-42 ${ }_{\text {hom }}$ correlates strongly with intraneuronal $A \beta_{4-42}$ accumulation in these mice. Tg4-42 and Tg4-42 ${ }_{\text {hom }}$ mice showed intracellular $A \beta$ beginning at two months. Strikingly, intraneuronal $A \beta$ in the $C A 1$ of the hippocampus showed strong immunoreactivity at two months of age which decreased with aging until it was almost absent at twelve months in Tg4-42 and eight months in Tg4-42 hom 
mice, respectively. The decrease can be attributed to neuronal death occurring in cells with $A \beta_{4-42}$ expression. It would be interesting to also determine the neuron number in the other brain areas expressing intraneuronal $A \beta$. Similar to Tg4-42, TBA2.1 transgenic mice expressing only $A \beta_{p E 3-42}$ develop a massive neuron loss in the CA1 of the hippocampus. $A$ similar pattern has been described in this mouse model as neuron loss in the CA1 region was associated with an reduced intraneuronal $A \beta$ signal (Alexandru et al., 2011).

In summary, it can be stated that the progressive neuron loss in the hippocampus of Tg4-42 and Tg4-42 hom mice correlates strongly with intraneuronal $A \beta_{4-42}$. The observations point to an crucial role of intraneuronal $A \beta$ in the process on neurodegeneration in $A D$. Furthermore, the results suggest that $N$-truncated $A \beta_{4-42}$ may play an important role in triggering neuron loss in $A D$ patients as $A \beta_{4-42}$ is highly abundant in the brain of $A D$ patients.

\subsubsection{Enhanced inflammation in Tg4-42 mice}

Neuroinflammation is a major pathological feature of $A D$ and occurs in pathologically vulnerable regions of the AD brain. Activated microglia and astrocytes have been detected in abundance near neurons and plaques and may contribute to the progression of $A D$ (Krause and Müller, 2010). Under physiological conditions, astrocytes act as 'multifunctional housekeeping' cells. Among others, they control local $\mathrm{pH}$ and ion concentrations, remove neuronal waste and provide general metabolic support to neurons. Astrocytes are activated in response to neuronal injuries (Li et al., 2011). When activated, microglia and astrocytes produce several pro-inflammatory signal molecules, including cytokines, growth factors, complement molecules and chemokines (Rubio-Perez and Morillas-Ruiz, 2012). It has been demonstrated that $A \beta$ is able to trigger astrocytes reactivation ( $\mathrm{Li}$ et al., 2011). Furthermore, a direct role of astrocytes in the degradation of abeta has also been proposed (Wyss-Coray et al., 2003). Under pathological conditions in $A D$ brains, neuroinflammatory processes become chronically active. Various inflammatory responses may contribute to the symptoms of $A D$ (Rebeck et al., 2010).

In several $A D$ mouse models, reactive astrocytes were found around plaques similar to the observations made in human AD patients. Moreover, the amount of gliosis increased simultaneously with the plaque load in Tg2576 and 5XFAD mice (Hsiao et al., 1996; Oakley et al., 2006). In contrast, TBA42 mice expressing $A \beta_{\mathrm{pE} 3-42}$ exhibited strong astrogliosis in the region of the hippocampus without showing plaques. The activation of astrocytes appeared in close proximity to the accumulation of $A \beta$ in these mice (Wittnam et al., 2012). Furthermore, in HOM TBA2.1 mice gliosis in the CA1 of the hippocampus corresponded closely to the $A \beta / A \beta_{p E}$ deposits and the observed neuron loss (Alexandru et 
al., 2011). Similar to these observations, activated astrocytes and microglia could be observed throughout the hippocampus in Tg4-42 and Tg4-42 hom mice. Mice showed increased astrogliosis with GFAP staining and microgliosis with IBA1 staining in the hippocampus as early as two months of age. Inflammation could be associated with the intracellular $A \beta$ accumulations in the hippocampus in these mice. The significant gliosis developed in the area of strong intraneuronal $A \beta$ immunoreactivity and neuronal loss in the absence of plauqes. These observations are an indicator for neuroinflammation associated neurodegeneration as it has been reported in several AD mouse models (Games et al., 1995; Sturchler-Pierrat et al., 1997). It should be noted that the activation of astrocytes and microglia precede the neuron loss in the hippocampus. However, it remains ambiguous if gliosis aggravates neuropathology through excitotoxic mechanism or whether it has an contrary protective role in A $\beta$ turnover (Domenici et al., 2002; WyssCoray et al., 2003; Block et al., 2007; Alexandru et al., 2011). In summary, the data suggest that intraneuronal $A \beta_{4-42}$ in vivo is a contributing factor to neuronal death as well as reactive gliosis in Tg4-42 mice.

\subsubsection{No weight loss in Tg4-42 mice}

Next to cognitive and behavioral deficits, $A D$ is frequently associated with nutritional disorders including weight loss and eating disorders. Epidemiologic studies have shown that weight loss appears in about $40 \%$ of patients (Guérin et al., 2005). It can occur in all stages of the disease but the odds of weight loss increase with the severity and progression of $A D$ and is especially common in end-stage $A D$ patients (Wallace et al., 1995; Gillette-Guyonnet et al., 2000; Tamura et al., 2007). AD-associated weight loss leads to reduced muscle mass and contributes to the decline of general health. It can ultimately contribute to increased risk of systemic infection, falls and loss of autonomy (Riviere et al., 2001; Guérin et al., 2005). The cause of weight loss in not clear yet but it seems multifactorial. Weight loss has also been detected in several AD mouse models. For example, nine months old 5XFAD mice showed a significant reduced body weight in comparison to their WT littermates (Jawhar et al., 2010). Weight loss has also been detected in HOM TBA2.1 and TASTPM mice (Pugh et al., 2007; Alexandru et al., 2011). In contrast, $\mathrm{Tg} 4-42$ and $\mathrm{Tg} 4-42_{\text {hom }}$ mice did not show any weight loss as they showed a comparable body weight to same-aged WT mice. Furthermore, the Tg4-42 and Tg4-42 hom experienced a similar body weight gain over time to WT mice. Previously, it has been reported that body weight significantly correlates with mice performance in the rotarod, a test that evaluates next to motor coordination grip strength and balance (Brown and Wong, 2007; Shiotsuki et al., 2010). It is conceivable that a weight loss accompanied with 
potential muscle weakness might also influence the outcome of motor tests including the balance beam and string suspension task. Therefore the lack of weight loss seems beneficial for analyzing motor function in mice.

\subsubsection{No motor deficits or anxiety-behavior in Tg4-42 mice}

Mice were subjected to a battery of behavior tests that included cognitive and noncognitive tests. The assessment of motor function in AD mice is a crucial aspect, as some reference memory task including the Morris Water maze are highly influenced by any disturbance in motor performance. Therefore motor impairments can result in deficient findings in the cognitive tests.

$A D$ is characterized by progressive cognitive impairments. In addition, patients develop neuropsychiatric symptoms including motor impairments, depression and agitation. Motor dysfunctions have been particularly observed in the late stages of $A D$. However, deficits have also been reported in about $10 \%$ of $A D$ patients in the early stages of the disease (Scarmeas et al., 2004; Wirths and Bayer, 2008). Sensory-motor abilities were analyzed in Tg4-42 mice using the balance beam and string suspension task. The balance beam task assesses fine motor coordinating and balance of mice (Hau and Schapiro, 2002; Luong et al., 2011). The string suspension task evaluates, besides motor coordination, grip and muscle strength of mice (Hullmann, 2012; Arendash et al., 2001b). Tg4-42 and Tg4-42 hom showed no motor deficits as they exhibited no deficits in grip strength, coordination or balance. Furthermore, motor performance declined slightly with age similar to WT mice.

It is crucial to observe motor function and anxiety behavior before assessing cognitive functions in $A D$ mice to avoid unwanted misinterpretations of the data. Several studies have described altered motor abilities in transgenic AD mice. APP/PS1KI as well as 5XFAD mice showed age-dependant motor impairments that are attributed to axonopathy (Wirths et al., 2008; Wirths and Bayer, 2008; Jawhar et al., 2010). In contrast, $3 \times \mathrm{Tg}$ mice performed superior than WT control animals in the rotarod test, a test also designed to test motor skills (Filali et al., 2012). Motor coordination impairments might be due to pathology in various CNS regions including spinal cord and cerebellum, areas that do not express $A \beta_{4-42}$ in Tg4-42 mice (Lalonde and Strazielle, 2007; Wirths and Bayer, 2008).

Furthermore, mice did not show a clasping phenotype. An abnormal clasping pattern has been observed in several mouse models including mice transgenic for mutant human APP and human four-repeat tau (Lalonde et al., 2012; Probst et al., 2000), as well as APP/PS1KI and 5XFAD mice. All of these mice showed signs of axonopathy (Tesseur 
et al., 2000; Wirths et al., 2007; Jawhar et al., 2010). Motor dysfunctions make it more difficult to study memory deficits, as several reference memory tasks including the Morris water maze can be highly influenced by impaired motor functions. Overall, Tg4-42 and Tg4-42 hom mice showed no signs for impaired motor abilities.

$A D$ patients can suffer from a wide range of neuropsychological symptoms including anxiety, disinhibition and depression (Mega et al., 1996; Lyketsos et al., 2002). Patients may be very withdrawn due to anxiety, while other patients may act totally disinhibited showing socially unacceptable behavior and inappropriate euphoria (Nash et al., 2007). The elevated-plus maze test is widely used to study anxiety-related behavior in mice. More time spend in the open arms of the maze reflects reduced anxiety and disinhibition, while less time spend in the open arms indicated increased anxiety levels (Walf and Frye, 2007). No anxiety related behavior deficits were seen Tg4-42 and $\mathrm{Tg} 4-42_{\text {hom }}$ at any age tested. Mice showed an equal age-dependant decreased anxiety behavior and decreased agitation with age as same-aged WT mice. Interestingly, AD mouse models showed inconsisted anxiety profiles. APP/PS1, 5XFAD and Tg2576 mice showed reduced anxiety-behavior shown by increased time spent in the open arms compared to same-aged WT animals. Whereas, other mouse models like 3xTg, APP23 and APP/PS1KI showed increased or unaltered anxiety levels (Lalonde et al., 2002; Espana et al., 2010; Webster et al., 2013). Elevated anxiety has been associated with intraneuronal $A \beta$ in the amygdala, an area that is not affected in Tg4-42 mice (Espana et al., 2010).

In summary, the results demonstrate that Tg4-42 and Tg4-42 $2_{\text {hom }}$ mice develop no deficits in either motor function or anxiety-behavior that could prevent or potentially confound memory testing of these mice.

\subsubsection{Age-dependant memory deficits in Tg4-42 mice}

Progressive memory decline is probably the most striking feature of AD. Therefore, a valid $A D$ mouse model should exhibit cognitive deficits that can be correlated with the ones observed in human AD patients. A wide range of cognitive tests are available to assess learning and memory abilities in mice.

Working memory in mice is widely investigated using maze type tasks like the $\mathrm{Y}$ maze, T-maze or cross maze. Working memory in rodents is related to spatial search strategies that are essential for foraging in the wilderness (Dember and Fowler, 1958; Dember and Richman, 1989). The cross maze task relays on the natural exploratory behavior of mice and is regarded as a rather rudimentary task to assess working memory. Alternation in the cross maze reflect the motivation of mice to explore an new 
environment. Healthy mice typically prefer to explore the least recently visited arm of the maze rather than returning to the one previously visited (Olton et al., 1979; Wietrzych et al., 2005). Age-dependant decrease in the spontaneous alternation has been observed in several AD mouse models including Tg2576, 5XFAD and 3xTg (Hsiao et al., 1996; Carroll et al., 2007; Jawhar et al., 2010). In contrast APP+PS1 mice showed even at 16 months of age no such phenotype (Arendash et al., 2001a). Tg4-42 and Tg4-42 ${ }_{\text {hom }}$ showed, irrespective of age, comparable spontaneous alternation rates to WT mice. The hippocampus is critically involved in spontaneous alternation and lesions in this brain area have been reported to alter the alternation behavior of rodents (Lalonde, 2002). However, despite a significant neuron loss in Tg4-42 and Tg4-42 ${ }_{\text {hom }}$ no impairments in the working memory version of the cross maze task could be detected in these mice. It has been speculated that the cross maze task fails to recognize hippocampal damage as hippocampectomized animals show side preferences. Such a preference, for example turning always left in a maze, could lead to high spontaneous alternation levels and therefore similar scores to WT animals (Deacon and Rawlins, 2006). Furthermore, next to the hippocampus other brain areas including the thalamus, substantia innominata and cerebellum are highly involved in spontaneous alternation (Lalonde, 2002).

In contrast, the Morris water maze (MWM) is a particularly sensitive test to examine age-related memory impairments in $A D$ mice. This task allows to assess hippocampus-dependant spatial learning and memory (Morris, 1984). In contrast to other memory test, such as fear conditioning and cross maze, nearly all well established $A D$ mouse models show age-related deficits the MWM. Mouse models carrying a single transgenic APP or tau mutation as well as mice carrying integrated APP and PSEN mutations or triple transgenic mice have been reported to display deficits in the MWM. APP23, Tg2576, 3xTg, 5XFAD and APP/PS1KI are only a few examples of mouse lines exhibiting spatial memory deficits (Westerman et al., 2002; van Dam et al., 2003; Billings et al., 2005; Webster et al., 2013; Bouter et al., 2014). The MWM consists of a large pool with a submerged platform in opaque water. Mice must learn to navigate to the hidden platform using spatial cues. This task is rather complex as it requires several cognitive processes simultaneously: adapting to a new and stressful situation, dropping ineffective search strategies, locating the escape platform based on spatial cues and encoding information to long-term memory (Tanila, 2012). The MWM allows to differentiate between spatial learning and long-term spatial reference memory. Furthermore, visual and motor performance can be evaluated in this behavior task, especially in the cued training. No differences were observed in Tg4-42, $\mathrm{Tg} 4-42_{\text {hom }}$ and WT mice in the cued training. Independently of age, mice demonstrated the appropriate skills to perform the MWM as they showed neither motor nor sensory deficits. The cued training is also striatum- 
dependant, a region that showed no neuron loss in Tg4-42 mice (Baldan Ramsey, Lissandra C. and Pittenger, 2010). Tg4-42 mice are not born with learning or memory deficits, as 3-month-old Tg4-42 and Tg4-42 hom mice display normal spatial reference memory. However, spatial memory was slightly impaired in the acquisition training in 8-month-old Tg4-42 hom and 12-month-old Tg4-42, respectively. Furthermore, the lack of a quadrant preference in the probe trial revealed spatial reference memory deficits in 8-month-old Tg4-42 ${ }_{\text {hom }}$ and 12-month-old Tg4-42 mice.

Reference memory is highly dependent on the hippocampus, one of the heavily affected brain region in AD patients (West, 1993). The observed impaired spatial memory in eight months old Tg4-42 $2_{\text {hom }}$ mice is accompanied by a severe neuron loss in the CA1 region (66\%). Despite a $38 \%$ neuron loss in the CA1 eight months old hemizygous Tg442 mice demonstrated no memory deficits in the MWM. Broadbent et al (Broadbent et al., 2004) examined the relationship between hippocampal lesion size and spatial memory in rats. Spatial memory impairment started after bilateral dorsal hippocampal lesions covering $30-50 \%$ of the total volume and as lesion size increased from $50 \%$ to $100 \%$ of total hippocampal volume, performance was similarly impaired. In addition, Moser et al (Moser et al., 1995) claimed that only $20-40 \%$ of the total hippocampus is required for efficient spatial learning. These findings demonstrate that the hippocampus is important for spatial memory albeit a significant neuron loss can be compensated. Next to the hippocampus additional brain structures have been reported to affect spatial memory by influencing movement organization, spatial navigation or motor performance. Among these structures are amygdala, prefontal cortex, striatum, cerebellum, thalamic structures and locus coeruleus (D'Hooge and De Deyn, P P, 2001; Puzzo et al., 2014). The results from Tg4-42 mice are in good agreement with these observations as a $38 \%$ neuron loss in the CA1 of the hippocampus in 8-month-old hemizygous Tg4-42 mice has no consequence on spatial reference memory performance. However, Tg4-42 hom mice with a $66 \%$ neuron loss demonstrate significant impaired spatial learning in the MWM. Both the age-dependent deficits in spatial reference memory and the severe hippocampal neuron loss in Tg4-42 $2_{\text {hom }}$ mice are compatible with AD-typical changes.

Twelve months old heterozygous Tg4-42 mice were also tested in the fear conditioning (FC) paradigm. $\mathrm{FC}$ is a form of associate learning that is based on conditioning (Puzzo et al., 2014). In patients with mild to moderate AD impaired conditioned fear responses have been shown (Hamann et al., 2002; Hoefer et al., 2008). Amygdala and hippocampus are crucial brain structures in FC learning. The amygdala is critical for the formation and storage of emotional memories, while the hippocampus is involved in remembering the context that triggered fear (Phillips and LeDoux, 1992; LeDoux, 2003; Sanders et al., 2003; Puzzo et al., 2014). Aged Tg4-42 exhibited an 
impaired context fear conditioning. The impairment is shown by the lack of freezing as a response to the context. In contrast, Tg4-42 mice exhibited a freezing response to the tone similar to WT mice. Freezing is a natural response mechanism of rodents to feign death in front of predators (Mongeau et al., 2003). Phillips and LeDoux (1992) reported that lesions of the hippocampus interfered with contextual fear conditioning but not with tone fear conditioning. In contrast, a functional amygdala is required for appropriate fear conditioning for both context and tone. These observations are well in line with the severe neuron loss detected in the hippocampus of twelve months old Tg4-42 mice and the lack of $A \beta$ expression in the amygdala. Consistent with the observations in Tg4-42 mice, several other AD transgenic models, including Tg2576, APP/PS1, 5XFAD and 3xTg, displayed impaired context fear conditioning (Corcoran et al., 2002; Billings et al., 2005; Knafo et al., 2009). 5XFAD also mice showed an impairment in tone conditioning (see section 3.2.4) (Bouter et al., 2014).

There is multiple evidence that soluble $A \beta$ oligomers plays a role in the memory impairment of $A D$ (Renner et al., 2010). It has been shown that soluble $A \beta_{42}$ oligomers and not plaque-associated $A \beta$ correlates best with the cognitive decline in $A D$ (McLean et al., 1999; Naslund et al., 2000). Furthermore, synaptic changes and memory deficits have been detected in several mouse models before plaque pathology (Holcomb et al., 1998). Oligomers are preferentially formed intracellular within neurons and synapses (Walsh et al., 2000; Takahashi et al., 2004). The mode of $A \beta$ toxicity, especially $A \beta_{4-42}$, is not clear to date (Bouter et al., 2013). Glabe and Kayed (2006) proposed that membrane permeabilization by amyloid oligomers may initiate a common group of downstream pathologic processes. These processes include production of reactive oxygen species, altered signaling pathways intracellular calcium dyshomeostasis and mitochondrial dysfunction that represent key effectors of cellular dysfunction and cell death (Glabe and Kayed, 2006). A oligomers can act as pathogenic ligands binding to particular synapses, disturbing their function, morphology and maintenance (Renner et al., 2010). Naturally secreted $A \beta$ have been shown to impair hippocampal long-term potentiating (Haass and Selkoe, 2007). Furthermore, it has been demonstrated that $A \beta_{1-42}$ oligomers induce the abnormal accumulation and over-stabilization of a glutamate receptor, thereby providing the molecular basis for $A \beta$ oligomer-induced early synaptic failure (Renner et al., 2010). As soluble and aggregated $A \beta_{4-42}$ is as toxic as $A \beta_{1-42}$ a similar mechanism might be true for $A \beta_{4-42}$

In conclusion, the behavior analysis of $\mathrm{Tg} 4-42$ and $\mathrm{Tg} 4-42_{\mathrm{hom}}$ mice revealed hippocampus-dependant memory deficits similar to AD patients. Tg4-42 and Tg4-42 hom are profoundly impaired in their spatial reference memory. Moreover, aged Tg4-42 showed a decline in contextual fear memory. The over-expression of $A \beta_{4-42}$ in this mouse 
model induces severe age-dependant memory deficits that can be attributed to the massive neuron loss in the hippocampus.

\subsection{8}

\section{Summary - Is Tg4-42 a valid model for AD?}

To date, a wide range of animal models of $A D$ have been generated and contributed to an better understanding of the pathogenesis of $A D$. Most of these models mimic certain aspects of the disease including amyloid plaques, NFTs, neuronal loss, gliosis or memory and cognitive deficits. However, so far no AD model represents the full range of pathological and degenerative alternations (Elder et al., 2010). For examples, as stated before, most transgenic mouse models expressing human APP show a robust plaque pathology but many of them lacking a significant neuron loss (Duyckaerts et al., 2008; Götz and Ittner, 2008; Cavanaugh et al., 2014). In most AD mouse models that do show neuron loss it is induced by a combination of multiple mutations that individually seem to be sufficient enough to cause AD in humans (Christensen et al., 2008; Breyhan et al., 2009; Elder et al., 2010; Jawhar et al., 2010). Strikingly, in Tg4-42 mice a massive neuron loss in the CA1 is induced by long-term exposure to $A \beta_{4-42}$ alone.

Despite being one of the major $A \beta$ isoforms in $A D$ brains, little has been known about its contribution to the etiology of AD (Portelius et al., 2010). The Tg4-42 mouse model expressing human $A \beta_{4-42}$ allows to investigate the neurotoxic effects of $A \beta_{4-42}$ in vivo. $\mathrm{N}$-truncated $A \beta$ peptides are very abundant in brains of patients diagnosed with sporadic and familial $A D$. Of the $N$-truncated species $A \beta_{4-x}$ and $A \beta_{p E 3-x}$ are the most consistently reported (Portelius et al., 2010; Bayer and Wirths, 2014). In many AD mouse model a accumulation of $\mathrm{N}$-terminal cleaved $A \beta$ was not been described. However, intraneuronal accumulation of $N$-truncated $A \beta$ has been linked to neuron loss in some $A D$ model including APP/PS1KI and 5XFAD mice. Moreover, $A \beta_{4-42}$ was the most abundant $\mathrm{N}$-truncated $A \beta$ form in 5XFAD mice (Bayer and Wirths, 2008; Wittnam et al., 2012).

$A D$ is a multifactorial and heterogeneous disease (lqbal et al., 2005b). Familial AD represent only a fraction of all $A D$ cases, still nearly all models for $A D$ are associated with FAD mutations. Around $99 \%$ of all AD cases are sporadic and the causes underlying these cases are still unclear (Zetterberg et al., 2014). There is an urgent need to generate disease-relevant SAD animal models, however so far there has been nearly no success in the generation of such animal models (lqbal et al., 2013). An animal model that shows at least some aspects of SAD has been generated by intracerebroventricular (icv) injection of streptozotocin (STZ). These rodents are generated based on the detection of an insulin-resistant brain state in SAD. STZ is injected to induce such a brain insulin resistant state. These icv-STZ mice show memory and learning deficits, decreased energy 
metabolism, neuroinflammation, altered synaptic proteins and increased hyperphosphorylated tau in the brain (Salkovic-Petrisic et al., 2006; de la Monte, Suzanne M. and Wands, 2008; Chen et al., 2012; Chen et al., 2013). The transgenic mouse model Tg4-42 is unique as it harbors no mutations in the $A \beta$ sequence. Moreover, the Tg4-42 mouse model can be considered as a model for sporadic $A D$, as no familial $A D$ mutation in $A \beta, A P P, P S E N-1$ or PSEN-2 is needed for inducing neurodegeneration in these mice. The over-expression of $A \beta_{4-42}$ induces gliosis, neuron loss and behavior deficits in Tg4-42 and Tg4-42 hom mice. Therefore, the Tg4-42 mouse model shows many aspects of an SAD brain, however they do not develop amyloid plaques or NFTs. Tg4-42 and Tg4-42 hom mice showed region-specific intraneuronal $A \beta$ deposits predominantly in the CA1 of the hippocampus. The expression of $A \beta_{4-42}$ was sufficient to induce neurodegeneration in Tg4-42 mice that is associated with gliosis and progressive and massive neuron loss in the hippocampus. Furthermore, $\mathrm{Tg} 4-42$ and $\mathrm{Tg} 4-42_{\text {hom }}$ mice develop an age-dependent hippocampus-related reference memory deficits in the Morris water maze due to the drastic CA1 neuron loss. Moreover, aged Tg4-42 show a decreased contextual learning in the fear conditioning task. Importantly, the observed memory deficits in Tg4-42 mice are not confounded by motor impairments or abnormal anxiety. Based on these findings, the Tg4-42 line represents a disease-relevant model of sporadic AD.

Despite being a major isoform in the brain of $A D$ patients, $A \beta_{4-x}$ has not received much attention as a potential therapeutic target. The massive neuron loss coupled with age- and disease related cognitive decline and the high physiological relevance of the Tg4-42 mouse model make it well-suited for utilization of preclicical testing of AD-relevant therapeutics. Intriguingly, the newly generated antibody NT4X-167 could be used for passive immunization of $\mathrm{Tg} 4-42$ mice. The antibody has been shown to significantly rescue $A \beta_{4-42}$ toxicity in vitro, while no beneficial effects were observed against $A \beta_{1-42}$ or $A \beta_{p E 3-42}$ toxicity (Antonios et al., 2013).

In summary, the pathology of Tg4-42 mice is unique in its massive neuron loss and severe memory behavior deficits induced through intraneuronal accumulation of $\mathrm{N}$-terminally truncated $A \beta_{4-42}$ (FIGURE 4.1). It can be stated that Tg4-42 mice emphasize the toxicity of $A \beta_{4-42}$ in vivo. Moreover, the resulting neurodegeneration associated with neuron loss, gliosis and memory deficits appears to be a direct consequence of intracellular neurotoxicity of $A \beta$. In summary, Tg4-42 is a valid AD mouse model showing key features of sporadic $A D$. 


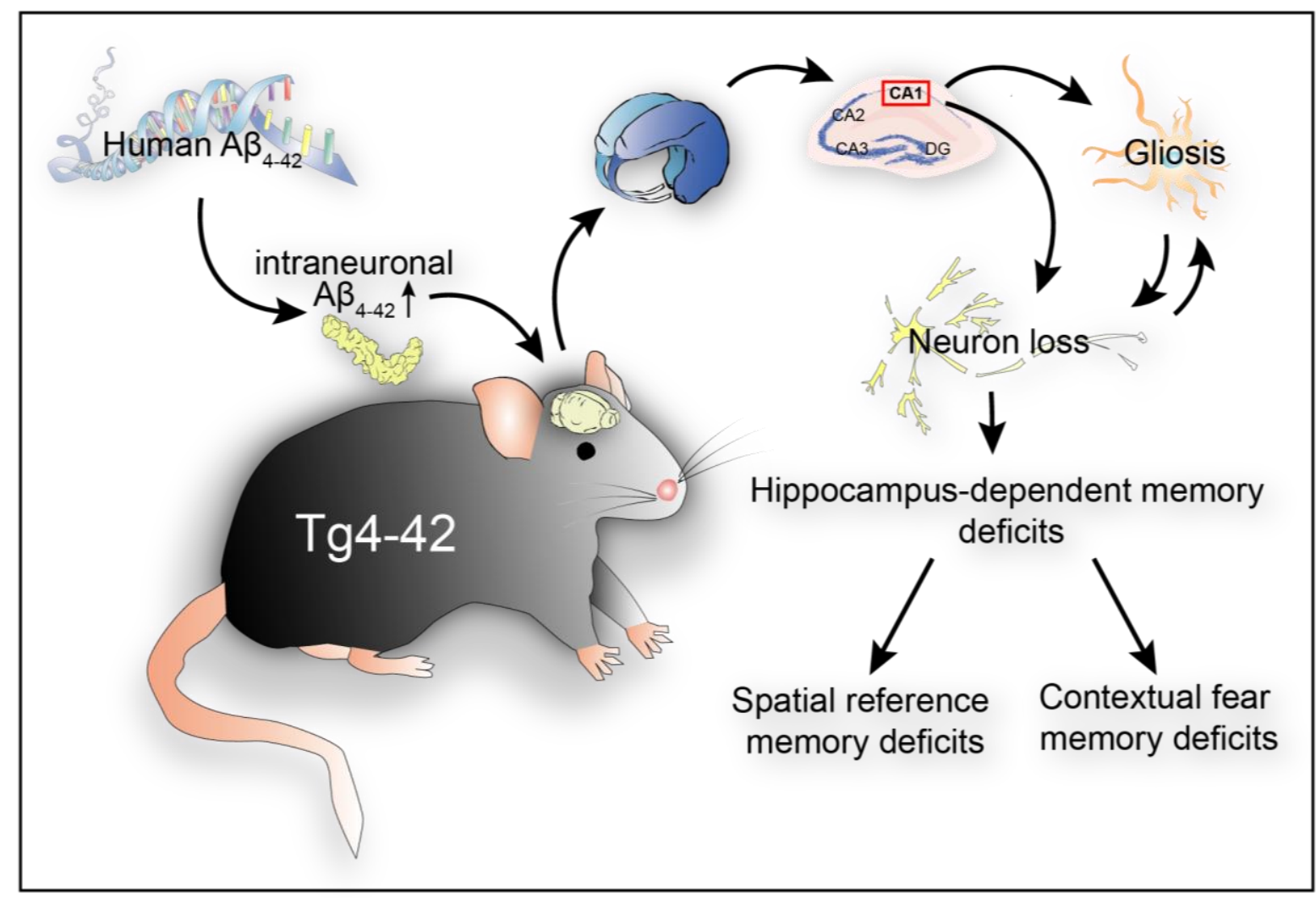

FIGURE 4.1 Key features of the Tg4-42 mouse model. Intraneuronal A 3 is expressed predominantly in the CA1 of the hippocampus. Tg4-42 mice develop a massive neuron loss in the CA1 and severe memory deficits induced through intraneuronal accumulation of $A \beta_{4-42}$. Inflammation could be associated with the intracellular $A \beta$ accumulations in the hippocampus in these mice. The significant gliosis developed in the area of strong intraneuronal $A \beta$ immunoreactivity and neuronal loss in the absence of plauqes. However, it remains unclear if gliosis aggravates neuropathology through excitotoxic mechanism or whether it has an contrary protective role in $A \beta$ turnover. 
The main objective of the second part of the thesis was to elucidate the similarities and distinctions in the expression profiles of the Tg4-42 and the 5XFAD mouse model. Therefore a comparative gene expression analysis of brain tissue of these $A D$ mouse models was performed using next-generation sequencing.

\subsection{1 $\quad$ Advantages of RNA Deep Sequencing}

The transcriptome includes all RNA transcripts expressed in a given tissue and reflects the genes that are expressed at the studied time point. Altered gene expression profiles may therefore provide information about the genes and mechanisms involved in the molecular pathogenesis of diseases like $A D$ and ultimately promote the search for new therapeutic drugs.

Microarrays have been used in the past as a standard process for transcriptome profiling. The method has been proven to be valuable to quantify simultaneously large numbers of mRNA transcripts (Courtney et al., 2010). Commercially available microarrays can analyze up to 15,000 - 30,000 different mRNAs and facilitate genome-wide gene expression profiling (Altar et al, 2009). Oligonucleotide and cDNA microarrays are both affordable and offer a high-throughput approach. However, due to the use of indirect signal detection by hybridization microarray techniques possess several limitations (Courtney et al., 2010). The limitations include reliance upon knowledge of already known sequences, poor range of quantification and relative low sensitivity and specificity (Choi et al., 2013). Furthermore, non-specific binding of samples make the detection of low expressed transcripts against the background noise difficult (Sutherland et al., 2011) and unsuitable for quantification of over- and under-expressed genes with fold changes smaller than two (Wang et al., 2009). Bakel and colleagues (2010) reported that hybridization signals from microarrays can lead to a high number of false positive signals especially from transcripts with low expression levels.

Several microarray studies on amyloid mouse models for $A D$ have been reported (Stein and Johnson, 2002; Dickey et al., 2003; Wu et al., 2006; Selwood et al., 2009; Wirz et al., 2013). The transgenic models included APP/PS1 $1_{\triangle \mathrm{E} \times 9}$, PDAPP, Tg2576 and combinations with different mutant PSEN-1 genetic variants. All of these models represent

\footnotetext{
${ }^{8}$ published in Bouter et al. (2014)
} 
models for familial $A D$ and abundant plaque formation without severe neuron loss. Therefore, in this study two models were compared that do show a robust behavioral deficit and in addition harbor a significant neuron loss.

RNA-Seq (deep-sequencing) allows to cope with many of the problems described for microarrays and has a number of advantages over microarray technology. Most importantly, deep sequencing does not rely on known genome sequence data and therefore novel transcripts can be detected (Courtney et al., 2010). It is possible to detect billions of nucleotide information within a single experiment (Cheng et al., 2013). Furthermore, problems with saturation and background signal do not exist as each molecule is individually sequenced and mapped to unique regions of the genome. RNASeq offers a larger dynamic range than microarray technology as no upper or lower levels exist in this quantification technique (Courtney et al., 2010). In comparison to microarrays deep sequencing has a low false positive rate and is moreover highly reproducible (Nagalakshmi et al., 2008).

\subsubsection{Comparison of Tg4-42 and 5XFAD mice}

Using deep sequencing technology the RNA profiles from the two AD models 5XFAD and Tg4-42 were analyzed (TABLE 4.1). These two models were compared as they show a robust behavioral deficit and develop a significant neuron loss.

5XFAD is a model for familial $A D$ that shows massive and early plaque formation, intraneuronal $A \beta$ aggregation, behavioral deficits and neuron loss in the cortical layer 5 (Oakley et al., 2006; Jawhar et al., 2010). In many aspects 5XFAD represents a very good $A D$ model. Due to the massive plaque formation in 5XFAD, many molecular pathways that are exclusively correlating to plaque formation may cover those pathways directly connected to neuron loss. Using mass spectrometric analysis, it could previously been demonstrated that 5XFAD mice exhibit high amounts of pyroglutamate $A \beta_{\mathrm{pE3}-42}$ and other $A \beta$ isoforms. Besides $A \beta_{1-42}$, the following peptides were also identified in 5XFAD mice, in order of abundance: $A \beta_{1-40}, A \beta_{4-42}$, pyroglutamate $A \beta_{p E 3-42}$ and $A \beta_{3-42}$ (Wittnam et al., 2012).

In vitro and in vivo analysis of amyloid deposits in $\mathrm{AD}$ revealed $\mathrm{N}$ - and $\mathrm{C}$-terminal variants of the $A \beta$ peptide (Masters et al., 1985; Prelli et al., 1988; Miller et al., 1993). Masters et al. (1985) discovered that the majority (64\%) of the peptides in amyloid plaques of $A D$ begin with a phenylalanine residue corresponding to position 4 of the fulllength sequence. Moreover, they detected dimeric and tetrameric $A \beta$ aggregates from the HPLC separations of plaques from $A D$ having the same ragged $\mathrm{NH}_{2}$-terminal ends. The importance of $A \beta_{4-42}$ was later supported by the finding that it represents a dominant 
fraction in the hippocampus and cortex of AD patients (Portelius et al., 2010). In addition, Lewis et al. (2006) reported that $A \beta_{4-42}$ is a relatively abundant species in $A D$, aged controls and vascular dementia patients.

In order to investigate the long-lasting neurotoxic effect of $A \beta_{4-42}$, the novel mouse transgenic mouse model Tg4-42 expressing exclusively $A \beta_{4-42}$ was recently generated (Bouter et al., 2013). The model is unique as it harbors no mutations in the $A \beta$ sequence and therefore it can be regarded as a model for sporadic AD. Tg4-42 mice develop severe hippocampal neuron loss and memory deficits that correlate well with the hippocampusspecific intraneuronal expression of $A \beta_{4-42}$ (see section 4.1.2). These findings are corroborated by previous mouse models expressing full-length mutant APP. For example, APP/PS1KI mice exhibit neuron loss in the CA1 region of the hippocampus (Casas et al., 2004; Breyhan et al., 2009), the frontal cortex (Christensen et al., 2008), and in distinct cholinergic nuclei (Christensen et al., 2010). The APP/PS1KI model is characterized by age-dependent accumulation of heterogeneous $\mathrm{N}$-terminal truncated $A \beta$ peptides with $A \beta_{4-42}$ being one of the most abundant variants (Casas et al., 2004). In 5XFAD mice a heterogeneous mixture of full-length, $\mathrm{N}$-truncated and modified $A \beta$ peptides, including $A \beta_{4-42}$, was found (Wittnam et al., 2012). Hence, the pathological events observed in the APP/PS1 $\mathrm{KI}$ and 5XFAD mouse models might be at least partly triggered by $\mathrm{N}$-terminal truncated $A \beta_{4-42}$.

TABLE 4.1 Comparison of the two transgenic mouse models 5XFAD and Tg4-42

\begin{tabular}{lll}
\hline Features & 5XFAD & Tg4-42 \\
\hline Mutations & APP695 (Swedish, Florida, London) & None \\
& PSEN-1 (M146L and L286V) & \\
Genetic background & C57BI6 & C57BI6 \\
Transient intraneuronal A $\beta$ & Yes & Yes \\
Prevalence of A $\beta$ variants & A $\beta_{1-42}>_{1-40}>{ }_{4-42}$ pyroglutamate3-42 $_{14}$ & only A $\beta_{4-42}$ \\
Plaques & Plaque deposits starting at 3 months & None \\
Neuron loss & $38 \%$ loss in cortical layer 5 & $49 \%$ loss in CA1 \\
Gliosis & Yes & Yes \\
Behavioral deficits & Yes & Yes \\
\hline
\end{tabular}




\subsection{3}

Learning and memory deficits in 5XFAD and Tg4-42 mice

In the present work it could be shown that Tg4-42 mice and 5XFAD mice feature comparable learning and memory deficits. Both mouse lines exhibited age-dependent spatial reference memory deficits assessed by the Morris water maze. Aged Tg4-42 and 5XFAD mice have also been tested in the contextual fear conditioning paradigm and exhibited deficits in this hippocampus-dependent memory task. Tg4-42 and 5XFAD mice displayed hippocampus-dependent memory deficits similar to those of other AD transgenic models (Chen et al., 2000; Stover and Brown, 2012; Kishimoto et al., 2013). Furthermore, 5XFAD mice demonstrated an impairment in conditioned learning in response to a tone stimulus.

Classical fear conditioning is assumed to be highly dependent on the hippocampus (Bast et al., 2003). Phillips and LeDoux (1992) reported that lesions of the hippocampus interfered with contextual fear conditioning but not with cue and tone fear conditioning. In contrast a functional amygdala is required for appropriate fear conditioning for both context and tone. Moreover, anxiety behavior was claimed to correlate with the presence of intraneuronal $A \beta$ in the amygdala (España et al., 2010). These observations are in agreement with the impairment in conditioned learning in response to a tone stimulus of aged 5XFAD mice, but not of age matched Tg4-42 animals.

\subsubsection{Deep sequencing in 5XFAD and Tg4-42 mice}

It could be shown that 5XFAD and Tg4-42 mice do develop comparable AD typical learning deficits at 12 months of age. Both mouse models also exhibit two cardinal features of $A D$ : accumulation of intraneuronal $A \beta$ and neuron loss (Oakley et al., 2006). In order to detect gene expression changes in the two $A D$ mouse models deep sequencing analysis was performed on young as well as aged 5XFAD and Tg4-42 mice. A wide range of differentially expressed genes (DEGs) could be identified in aged Tg4-42 as well as in young and aged 5XFAD mice compared to age-matched wild-type controls, respectively. Even though the potential for false positive results cannot be eliminated completely, more than 25 transcript changes detected by RNA-Seq could be successfully confirmed by qRT-PCR and therefore validated the deep sequencing results. Furthermore, the detection of the transgenic human PSEN-1 and APP sequences in young and aged 5XFAD mice as well as the promoter sequences through deep sequencing is also an indication for the quality of the method.

The expression changes detected in the transgenic mice give a broad picture of the profound physiological changes that accompany the neuron loss and the detected memory deficits in 5XFAD and Tg4-42 mice. Some of the DEGs have been reported 
before, while many genes are described for the first time in the context of AD. The observed parallel expression of these genes now offers new perspectives in understanding the pathology of $A D$.

\subsubsection{Differentially expressed genes in young 5XFAD and Tg4-42 mice}

In young 5XFAD mice a substantial number of genes is differentially expressed prior to robust amyloid deposition and neuron loss. The 19 DEGs encoded proteins from diverse functional categories, including translation, glycolysis and ATP-binding, kinases and hydrolases. The 5XFAD model has been reported to develop plaque deposition starting already at the age of 3 months (Jawhar et al., 2010). Intraneuronal $A \beta$ is evident at 1.5 months of age, just before the first appearance of amyloid deposits (Oakley et al., 2006). The data of young 5XFAD mice elucidate the expression profile at the commencement of plaque formation, before learning and memory deficits are apparent. Several DEGs that are involved in the clearance of $A \beta$. For example, transthyretin (Ttr) (Li and Buxbaum, 2011) and insulin-degrading enzyme (Ide) (Farris et al., 2003; Miners et al., 2009) are found up-regulated. The serum- and glucocorticoid-inducible-kinase-1 (SGK1) participates among others in the regulation of neuroexcitability, inflammation, cell proliferation and apoptosis. It is assumed that it takes part in the signaling of brain-derived neurotrophic factor (BDNF) which is involved in neuronal survival and plasticity (Lang et al., 2010).

No DEGs were detected in young Tg4-42 mice, which suggests that the pathology is weak at that age and points to a later onset of the pathological events underlying the phenotypic changes observed at later ages.

\subsubsection{Common molecular signature of Tg4-42 and 5XFAD mice}

Interestingly, 36 genes were differentially expressed in both mouse models indicating common disease pathways associated with behavioral deficits and neuron loss occurring in these mouse models. Nearly half of the DEGs in aged Tg4-42 were also differentially expressed in 5XFAD mice.

Many of the genes that showed differential regulation in 5XFAD alone belong to neuroinflammatory processes typically found associated with plaques. As Tg4-42 mice do not develop any plaques, but massive neuron loss, it can be assumed that the genes isolated in both models and those in Tg4-42 alone are defining the molecular signature underlying memory decline in $A D$.

The DEGs that were found in both models fall in a broad range of functional categories: regulation of cell differentiation and anatomical structure development, 
regulation of gene expression and transcription, histone modification, ion binding and protein methyltransferase activity, nervous system development and neurogenesis. Together with Calm3, Fbxo2 and Gpm6a only Gfap was found to be up-regulated in both aged 5XFAD and Tg4-42 mice. The astrocyte marker glial fibrillary acidic protein gene (Gfap) was found to be similarly up-regulated in both mouse lines. Increased astrogliosis was previously described in both mouse lines (Bouter et al., 2013; Oakley et al., 2006). Increased astrogliosis, measured by GFAP concentration, is also found in cortex, thalamus, brainstem and cerebellum in AD brains (Delacourte, 1990).

Next to the up-regulated genes 32 genes were commonly down-regulated in aged transgenic mice compared to same-aged WT mice. Among others Lrp1 was altered. Kanekiyo et al. (Kanekiyo et al., 2013) demonstrated that receptor-mediated endocytosis in neurons by LRP1 plays a critical role in A $\beta$ clearance in the brain. Decreased levels of Shank1 RNA were also found in both mouse lines. The levels of the postsynaptic proteins SHANK1 and SHANK3 are also regulated in patients with Alzheimer's disease and in the brains of amyloid precursor protein transgenic mice. It has been proposed that $A \beta$ reduces Shank levels in the dendrites (Pham et al., 2010).

The gene coding for the lysine (K)-specific methyltransferase 2D (MII2), also known as $K m t 2 b$, that is highly expressed throughout development as well as in adult tissue (Glaser et al., 2006) is down-regulated in aged 5XFAD and Tg4-42. Kerimoglu et al. (Kerimoglu et al., 2013) showed that mice lacking MII2 in the adult forebrain displayed impaired hippocampus-dependent memory function. Furthermore, the loss of MLL2 leads to down-regulation of genes implicated in neuronal plasticity. 5XFAD and Tg4-42 also showed hippocampus-dependent memory impairments. The down-regulation of MII2 that is reported to be crucial for memory consolidation and regulation of hippocampal plasticity genes is well in line with these findings.

Besides the RNA binding protein neuro-oncological ventral antigen 2 gene, Ubiquilin 2 (Ubqln2) was similarly down-regulated in aged Tg4-42 and both young and aged 5XFAD mice. UBQLN2 is a member of the ubiquilin family that is involved in the protein-degrading pathway as it regulates the degradation of ubiquitinated proteins (Ko et al., 2004). Ubiquilin has recently been linked to intraneuronal inclusions in amyotrophic lateral sclerosis (ALS), a rapidly progressive motoneuron disease. Around $20 \%$ of ALS patients also develop a frontotemporal dementia (Deng et al., 2011). Noticeable, in the hippocampus of patients with sporadic and familiar ALS ubiquilin 2 pathology correlates with dementia (Deng et al., 2011). Furthermore, ubiquilin 1 and ubiquilin 2 protein levels have been shown to be reduced in the brains of AD patients (Stieren et al., 2011; Nölle et al., 2013). It could be shown that ubiquilin 1 modulates $y$-secretase cleavage (Viswanathan et al., 2011) and that down-regulation of ubiquilin 1 results in an increase of 
amyloid precursor protein (APP) fragments (Stieren et al., 2011). It was suggested that ubiquilin 2 decline contributes to the AD pathology via APP processing as well and that ubiquilin 2 may be generally involved in the pathology of neurodegenerative dementias (Nölle et al., 2013).

\subsubsection{Differentially expressed genes in aged Tg4-42 mice}

Up-regulated genes in aged Tg4-42 mice

Twenty genes were solely differentially expressed in aged Tg4-42. Among these, only the genes Uqcc2, Beta-S and Kif1a were found to be up-regulated. Kif1a is a member of the kinesin family (KIFs) (Takemura et al., 1996) and has previously been connected to AD (Kondo et al., 2012). These microtubule-based motor proteins transport membrane organelles, mRNA and proteins (Hirokawa et al., 2009). By transporting those complexes KIFs play important roles in neuronal function and plasticity as well as morphogenesis and survival (Hirokawa et al., 2010). In neurons KIF1A transports components of synaptic vesicles containing synaptic vesicle proteins such as synaptophysin and synaptotagmin (Hirokawa et al., 2010). Recently, Kondo et al. (2012) showed that an up-regulation of KIF1A contributes to synaptogenesis in the hippocampus.

\section{Down-regulated genes in aged Tg4-42 mice}

The 17 genes that were significantly down-regulated in aged Tg4-42 are involved in diverse biological processes. These include regulation of gene expression, nervous system development, cell communication, metal ion transport, neurogenesis and regulation of synaptic plasticity. The gene encoding the nerve growth factor inducible protein (VGF), which is down-regulated in aged Tg4-42, is a neurosecretory protein that is solely expressed in neurons (van den Pol et al., 1994). Adult VGF is detected in several areas in the brain including the olfactory system, cerebral cortex, hypothalamus and hippocampus as well as the adrenal medulla and motorneurons of the spinal cord (van den Pol et al., 1994; Snyder and Salton, 1998; Thakker-Varia and Alder, 2009). Several groups proposed VGF as a potential biomarker for AD (Carrette et al., 2003; Jahn et al., 2011). They detected lower protein levels of VGF in the cerebrospinal fluid (CSF) of AD patients compared to healthy controls. Recently, it has been proposed that VGF plays a role in the regulation of energy balance as knockout mice are hypermetabolic and thin (Levi et al., 2004). It could be speculated that the down-regulation of VGF is connected to the weight loss observed in some AD patients (Carrette et al., 2003). Tg4-42 mice however did not display any weight loss (section 3.1.3.4.1). 
Another notable down-regulated gene in aged Tg4-42 mice codes for doublecortin (Dcx). Doublecortin is a microtubule-associated protein that is expressed in migrating neuronal precursors of the developing CNS and immature neurons (Couillard-Despres et al., 2005). DCX is often used as a marker for neurogenesis (Couillard-Despres et al., 2005; Verwer et al., 2007). In AD mouse models expressing mutant forms of APP or PSEN-1 neurogenesis was found to be impaired. $A \beta$ was found to disrupt neurogenesis in the subventricular zone and the hippocampus in these mice (Haughey et al., 2002a; Haughey et al., 2002b). Jin et al. (2004) however described increased levels of doublecortin in the hippocampus of $A D$ patients brains and therefore suggested that neurogenesis is increased in AD hippocampus.

Furthermore, the pathology of $A D$ has recently been linked to the deregulation of cyclin-dependent kinase 5 (CDK5) (Shukla et al., 2012). CDK5 is regulated by the neuronspecific cyclin-related proteins p35 (CDK5R1) and p39 (CDK5R2). Activated CDK5 plays an important role in neurogenesis, synaptic plasticity and neuronal survival (Nikolic et al., 1996; Tan et al., 2003; Shukla et al., 2012). CDK5 phosphorylates tau and the CDK5 complex is involved in posttranslational modification of APP and PSEN (Rademakers et al., 2005). Various neurotoxic events, including oxidative stress and elevated $A \beta$ levels, result in calpain cleavage of the regulatory proteins p39 and p35. The resulting C-terminal truncated proteins p29 and p25 lead to hyperactivation and mislocalization of CDK5. The introduction of p25 in primary neurons leads to the deregulation of CDK5 causing, among others, phosphorylation of tau and neuronal cell death (Cruz and Tsai, 2004; Rademakers et al., 2005). It can be hypothesized that the over-expression of $A \beta_{4-42}$ in Tg4-42 mice stimulates activation of calpain and therefore down-regulation of Cyclin-dependent kinase 5 activator 2.

It is noticeable that several DEGs in aged Tg4-42 mice have an ion binding function. The proteins ZMIZ2 and ZFP609 bind to zinc ions while Beta-S is an iron ion binding protein. Furthermore, the metal ion binding proteins MLL1, ZFHX3, SRRM2 and ATP1A are down-regulated in both aged Tg4-42 and 5XFAD mice. The binding targets zinc and iron, in addition to copper, have been shown to be involved in the pathology of $A D$. Zinc promotes the aggregation of $A \beta$ (Watt et al., 2010) and was found to be enriched in AD plaques (Lovell et al., 1998; Leskovjan et al., 2011; Roberts et al., 2012). While the overall zinc level in the aging brain is relatively constant, the zinc transporter ZnT3 has been shown to decrease with age (Roberts et al., 2012). Furthermore, disruption of zinc homeostasis in the brain leads to synaptic and memory deficits (Watt et al., 2010). Aged 5XFAD mice also displayed a variety of DEGs involved in metal binding, for example Bsn, Rnf44, Rai1, Atp1a2 and Rnf165. 


\subsection{8}

Differentially expressed genes in aged 5XFAD mice

In aged 5XFAD mice 131 genes with significant expression changes were identified. 87 of these genes were only found to be altered in this mouse line and not in Tg4-42. Compared to aged Tg4-42 mice a significant larger number of genes were differentially expressed in aged 5XFAD mice.

\section{Inflammatory processes}

A large number of DEGs are involved in regulation of immune system processes and inflammation. The respective transcripts are involved, among others, in adaptive immune response, regulation and activation of immune response as well as immune system development. Inflammatory processes in the brain are a well-described feature of AD. It has been shown that plaque deposition in $A D$ brains is associated with chronic inflammation characterized by increased inflammatory cytokine expression and activation of microglia, astrocytes and complement factors (Akiyama et al., 2000). Inflammation is thought to be a downstream process appearing after $A \beta$ plaques, NFT and neuron degeneration (Arnaud et al., 2006). 5XFAD mice display distinct neuroinflammatory features. The number of reactive astrocytes and microglia increases proportionally to the amyloid burden in this mouse line (Oakley et al., 2006; Kalinin et al., 2009).

5XFAD mice also show a dramatic increase in $A \beta_{42}$ in comparison to $A \beta_{40}$. This results in an early pathology onset with plaque deposition seen as early as three months of age. The plaque pathology increases dramatically in an age-dependent manner (Oakley et al., 2006; Jawhar et al., 2010). Aggregation of $A \beta$ results in activated microglia and induces the production of reactive-oxygen species, pro-inflammatory cytokines, chemokines and prostaglandines leading to degenerative changes in neurons (Akiyama et al., 2000). A large number of DEGs in aged 5XFAD mice have a role in inflammatory pathways (including Scube1 and Nr1d1). Strikingly, four genes of the complement system (C4b, C1qa, C1qb and C1qc) are highly up-regulated in 12-month-old 5XFAD mice. Complement activation is a major inflammatory process and is thought be activated in $A D$ by the interaction of complement proteins with the aggregated forms of $A \beta$ and tau (Rogers et al., 1992; Shen et al., 2001). Furthermore, it is assumed that soluble, nonfibriallar $A \beta$ peptides are also able to activate the complement system but to a smaller extent (Bergamaschini et al., 1999). The activation of complement enhances other toxic processes (Loeffler et al., 2006).

Notably, five genes, encoding the cysteine proteases Cathepsin B, Cathepsin L, Cathepsin S and Cathepsin Z as well as the aspartyl protease Cathepsin D, were up- 
regulated in aged 5XFAD mice. Cathepsin $D$ is a lysosomal enzyme found in neuritic plaques and is considered to be involved in APP processing (Schuur et al., 2011). Cataldo et al. (Cataldo et al., 1995) showed an up-regulation of Cathepsin D mRNA in the pyramidal neurons of $A D$ brains. The cysteine protease Cathepsin $B$ has been proposed as an alternative candidate $\beta$-secretase in the regulated secretory pathway of neurons, where it produces A $\beta$ by cleavage of the WT $\beta$-secretase site of APP (Hook et al., 2009; Wang et al., 2012). Hook et al. (2009) demonstrated that deletion of Cathepsin B in a hAPPwt transgenic mouse model significantly reduced the levels of $A \beta_{40}$ and $A \beta_{42}$. Therefore, Cathepsin $B$ might be a valid target for developing inhibitors to lower brain $A \beta$ levels in AD patients.

Another interesting gene that showed an up-regulated expression in aged 5XFAD mice is clusterin $(\mathrm{Clu})$ also known as apolipoprotein $\mathrm{J}$. Clusterin is a chaperone glycoprotein that affects many cellular processes, including inflammation. Clusterin is elevated in AD affected brain regions and CSF from AD patients (Lidström et al., 1998; Nilselid et al., 2006). Furthermore, it was found to be associated with $A D$ in several large genome-wide association studies (GWAS) (Harold et al., 2009; Lambert et al., 2009; Carrasquillo et al., 2010). Recent studies suggest that Clusterin contributes to the pathology to $A D$ through various pathways, including lipid metabolism, neuroinflammation and apoptosis. Interestingly, it is reported to increase $A \beta$ aggregation as well as $A \beta$ clearance (Yu and Tan, 2012).

It should be noted that Inpp5d RNA was found to be differentially expressed in 5XFAD mice. This gene was recently described as a new locus for $A D$ in a GWAS (Lambert et al., 2013). Inpp5d encodes a member of the inositol polyphosphate-5phosphatase family of enzymes involved in second messenger signaling in myeloid cells. INPP5D influences pathways that are associated with cell proliferation and inflammatory responses (Medway and Morgan, 2014).

\section{Evidence for diverse molecular pathways}

In addition to genes involved in inflammatory processes, DEGs in aged 5XFAD mice were also involved in cell communication and system development, signal transduction, synaptic transmission as well as regulation of gene expression and transcription. Significant transcriptional changes of genes with synaptic function in aged 5XFAD mice could be observed. For instance, the gene products of Bsn, Nrxn2, Kcnc1, Grm2 and Gng4 all play a role in synaptic transmission and are down-regulated in 12-month-old 5XFAD mice. Syn1, the gene encoding Synapsin1, a neuronal phosphoprotein associated with the cytoplasmic surface of synaptic vesicles, is significantly down-regulated in aged 
5XFAD mice. It is involved in synapse formation and promotion of neurotransmitter release (Südhof, 1990; Jaffrey et al., 2002). Qin et al. (2004) showed that synapsin levels were also significantly decreased in the CA1 and the dentate gyrus in AD patients.

Wirz et al. (2013) studied the genome-wide gene expression of another AD double transgenic APP/PS1 mouse model using microarrays. A vast range of genes was altered in these $A P P / P S 1_{\triangle E \times 9}$ mice that are involved in immune response and inflammation. In contrast to the observations in 5XFAD mice, no changes in the expression of genes involved in synaptic plasticity or transmission were found. However, in AD patients dominant gene expression changes concerning synaptic plasticity or transmission were recently described in a genome-wide gene expression study of the prefrontal cortex (Bossers et al., 2010). It can be argued that deep sequencing and the use of 5XFAD mice are more informative and better suited to identify the expression changes in a model system of $A D$.

\subsubsection{Limitations of the study}

Finally, it can be stated that RNA-Seq is a powerful technique to analyze the expression profiles in $A D$ mice. The detection of hundreds of differentially expressed genes may offer a new perspective on the biological processes underlying the pathology of AD. However, even though there is a strong correlation between gene expression levels and abundances of the respectively corresponding proteins in mammalian cells (Lundberg et al., 2010), it has to be kept in mind that proteins, rather than mRNAs, are the main mediators of physiological processes and that there is a considerable body of data that suggests a major role for post-transcriptional processes in controlling protein abundances (Vogel and Marcotte, 2012).

While investigating the role of DEGs on the protein level is beyond the scope of this study, the presented dataset could provide an important source of information for the validation in both mouse and human tissue in independent studies. A wide range of detected genes were previously shown to be regulated in AD, however, a variety of DEGs in the studied mouse models were not previously associated with $A D$ in humans. It remains to be seen if these genes are also regulated in $A D$ cases.

\subsubsection{Conclusions}

In conclusion, (1) the Tg4-42 model could be validated as a valuable model for AD. The comparison with 5XFAD, an established plaque-developing AD mouse model, revealed a remarkable overlap in the molecular signature. The jointly differentially expressed genes might indicate common pathways that are involved in the comparable learning and 
memory decline apparent at twelve months of age in both transgenic models. (2) The pool of genes that showed differential expression exclusively in Tg4-42 is likely associated to soluble $A \beta$ as no extracellular plaques are found in this model. In addition, the robust CA1 neuron loss could also contribute to the differential expression profile. (3) As most of the genes with expression levels exclusively altered in 5XFAD mice belong to inflammationassociated pathways, it can be concluded that the majority of these genes are regulated in connection with plaque formation in this model and are not associated with neuron loss and memory decline. (4) As expected the deep sequencing approach identified a plethora of genes that have so far not been linked to $A D$, which might opens up new avenues of research into the etiology of this devastating neurodegenerative disorder. 
Besides $A \beta$ staring with an aspartate at position 1, a variety of modified and truncated $A \beta$ forms have been detected in $A D$ brains. Together with full length $A \beta_{1-42}$ and $A \beta_{1-40}$, $N$-truncated $A \beta p_{E 3-42}$ and $A \beta_{4-42}$ are major variants in $A D$ (Bayer and Wirths, 2014). $A \beta_{4-42}$ has been identified as a particular abundant $A \beta$ species in the hippocampus and cortex of AD patients and was discovered as early as 1985 (Masters et al., 1985; Portelius et al., 2010). Sedimentation studies indicate that $A \beta_{4-42}$ exhibits fast aggregation kinetics (Pike et al., 1995b). Interestingly, short-term treatment of primary cortical neurons revealed that $A \beta_{4-42}$ is as toxic as pyroglutamate $A \beta_{3-42}$ and $A \beta_{1-42}$. Moreover, treatment of wild-type mice using intraventricular $A \beta$ injection with $A \beta_{4-42}$ induced significant working memory deficits. These deficits were similar to the ones induced through pyroglutamate $A \beta_{3-42}$ and $A \beta_{1-42}$ (Bouter et al., 2013). However, relatively little is known about the contribution of $A \beta_{4-42}$ to the development and progression of $A D$. In this study, it could be shown that $A \beta_{4-x}$ precedes $A \beta_{p E 3-x}$ accumulation in the brain of young 5XFAD mice by using the novel antibody NT4X-167. Moreover, $A \beta_{4-x}$ could be detected together with $A \beta_{1-x}$ intraneuronal in cortical neurons of six weeks old homozygous SXFAD mice. The results emphasize the significance of $A \beta_{4-42}$ in $A D$ pathology.

In the present work, the effects of chronic exposure of $A \beta_{4-42}$ in vivo were studied. Nearly all transgenic mouse models for $A D$ rely on artificial combinations of mutations. However, the vast majority of $A D$ patients do not possess mutations and suffer from the sporadic form of the disease (Elder et al., 2010; Zetterberg and Mattsson, 2014). Therefore, direct expression of a specific $A \beta$ species in vivo may demonstrate a more physiologically relevant AD model. Given these considerations the Tg4-42 mouse model, that exclusively expresses human $A \beta_{4-42}$, was recently generated in our lab. (Bouter et al., 2013). Here, the new homozygous Tg4-42 line was generated ( $\mathrm{Tg} 4-42_{\text {hom }}$ ) in addition to heterozygous mice. Using immunohistochemistry, it could be shown that Tg4-42 and Tg4-42 $2_{\text {hom }}$ mice develop region-specific intraneuronal accumulation of $A \beta$ accompanied by gliosis. Tg4-42 and Tg4-42 ${ }_{\text {hom }}$ mice showed strong intraneuronal $A \beta$ immunoreactivity predominantly in the CA1 pyramidal cell layer of the hippocampus beginning at two 
months of age. Intraneuronal $A \beta$ was also detected in Tg4-42 mice in the occipital cortex, piriform cortex, striatum and superior colliculus starting a two months of age. $A \beta_{4-42}$ expression in the CA1 declined during aging in Tg4-42 and Tg4-42 ${ }_{\text {hom }}$ mice. The decrease of $A \beta$ expression can be attributed to the extensive neuron loss in this brain region. Longterm exposure to $\mathrm{N}$-truncated $A \beta_{4-42}$ induced an age- and dose-dependent neuron loss in hemizygous and homozygous Tg4-42 mice in the CA1 layer of the hippocampus. At eight months unbiased stereology revealed a $38 \%$ neuron loss in hemizygous Tg4-42 mice and a $65 \%$ neuron loss in Tg4-42 hom. Furthermore, 12-month-old Tg4-42 mice showed a $51 \%$ decrease in neuron number in the CA1 compared to same-aged WT.

The behavior analysis of $\mathrm{Tg} 4-42$ and $\mathrm{Tg} 4-42_{\text {hom }}$ mice revealed that mice develop no deficits in either motor function or anxiety-behavior that could prevent or potentially confound memory testing of these mice. Strikingly, mice showed hippocampus-dependant memory deficits similar to AD patients. $\mathrm{Tg} 4-42$ and $\mathrm{Tg} 4-42_{\text {hom }}$ were profoundly impaired in their spatial reference memory. Moreover, aged Tg4-42 showed a decline in contextual fear memory. The over-expression of $A \beta_{4-42}$ in this mouse model induces severe agedependant memory deficits that can be attributed to the massive neuron loss in the hippocampus.

In summary, the pathology of Tg4-42 mice is unique in its massive neuron loss and severe memory behavior deficits induced through intraneuronal accumulation of $\mathrm{N}$-terminally truncated $A \beta_{4-42}$ albeit without plaque formation. It can be stated that $\mathrm{Tg} 4-42$ mice emphasize the toxicity of $A \beta_{4-42}$ in vivo. Moreover, the resulting neurodegeneration associated with neuron loss, gliosis and memory deficits appears to be a direct consequence of intracellular neurotoxicity of $A \beta$. In summary, Tg4-42 is a valid AD mouse model showing key features of sporadic AD.

One of the central research questions on the etiology of $A D$ is the elucidation of the molecular signatures triggered by the amyloid cascade of pathological events. Next generation sequencing allows the identification of genes involved in disease processes in an unbiased manner. A comparative gene expression analysis of brain tissue of Tg4-42 and 5XFAD mice was performed using next-generation sequencing. As stated before, Tg4.42 mice express $N$-truncated $A \beta_{4-42}$ and develop intraneuronal $A \beta$ aggregation, neuron loss and behavioral deficits albeit without plaque formation. The widely used 5XFAD model, which is based on the expression of mutant amyloid precursor protein and presenilin-1 genes, is a typical model for early plaque formation, intraneuronal $A \beta$ aggregation, neuron loss and behavioral deficits (Oakley et al., 2006; Jawhar et al., 2010). Interestingly, the results showed that learning and memory deficits in the Morris water maze and fear conditioning tasks in Tg4-42 mice at twelve months of age are similar to 
the deficits in 5XFAD animals. This suggested that comparative gene expression analysis between the models would allow the dissection of plaque-related and -unrelated disease relevant factors. Therefore brain transcriptomes of young and old Tg4-42, 5XFAD, and wild-type control mice were analyzed by deep-sequencing of non-ribosomal RNA (RNASeq). Differentially expressed genes represented by more than 200 deep sequencing reads (DEGs) were verified by qRT-PCR. Nineteen DEGs were identified in presymptomatic young 5XFAD mice, while no DEGs could be isolated in young Tg4-42 mice. In the aged cohort, 131 DEGs were found in 5XFAD and 56 DEGs in Tg4-42 mice. Intriguingly, 36 DEGs were identified in both mouse models indicating common disease pathways associated with behavioral deficits and neuron loss. The pool of genes that showed differential expression exclusively in Tg4-42 is likely associated to soluble $A \beta$ as no extracellular plaques are found in this model. In addition, the robust CA1 neuron loss could also contribute to the differential expression profile as 5XFAD mice display no neuron loss in this brain region. Many of the DEGs specific to the 5XFAD model belong to neuroinflammatory processes typically associated with plaques. As Tg4-42 mice do not develop any plaques, but still show massive neuron loss, it can be concluded that only the DEGs common to both models together with those specific to Tg4-42 are defining the molecular signature underlying memory decline in AD.

Taken together, the results of this thesis demonstrate $A \beta_{4-42}$ as a toxic $A \beta$ variant that likely plays a dominant role in triggering $A D$ pathology. 
Abramowski, D., Rabe, S., Upadhaya, A.R., Reichwald, J., Danner, S., Staab, D., Capetillo-Zarate, E., Yamaguchi, H., Saido, T.C., and Wiederhold, K.-H., et al. (2012). Transgenic expression of intraneuronal Abeta42 but not Abeta40 leads to cellular Abeta lesions, degeneration, and functional impairment without typical Alzheimer's disease pathology. J Neurosci 32, 1273-1283.

Akiyama, H., Barger, S., Barnum, S., Bradt, B., Bauer, J., Cole, G.M., Cooper, N.R., Eikelenboom, P., Emmerling, M., and Fiebich, B.L., et al. (2000). Inflammation and Alzheimer's disease. Neurobiol. Aging 21, 383-421.

Albert, M.S., DeKosky, S.T., Dickson, D., Dubois, B., Feldman, H.H., Fox, N.C., Gamst, A., Holtzman, D.M., Jagust, W.J., and Petersen, R.C., et al. (2011). The diagnosis of mild cognitive impairment due to Alzheimer's disease: recommendations from the National Institute on Aging-Alzheimer's Association workgroups on diagnostic guidelines for Alzheimer's disease. Alzheimers Dement 7, 270-279.

Aldwin, C., and Gilmer, D. (2013). Health, Illness, and Optimal Aging, Second Edition: Biological and Psychosocial Perspectives (Springer Publishing Company).

Alexandru, A., Jagla, W., Graubner, S., Becker, A., Bäuscher, C., Kohlmann, S., Sedlmeier, R., Raber, K.A., Cynis, H., and Rönicke, R., et al. (2011). Selective hippocampal neurodegeneration in transgenic mice expressing small amounts of truncated $A \beta$ is induced by pyroglutamate- $A \beta$ formation. J. Neurosci. 31, 12790-12801.

Almeida, C.G., Takahashi, R.H., and Gouras, G.K. (2006). Beta-amyloid accumulation impairs multivesicular body sorting by inhibiting the ubiquitin-proteasome system. J Neurosci 26, 4277-4288.

Alonso, A.C., Grundke-lqbal, I., and lqbal, K. (1996). Alzheimer's disease hyperphosphorylated tau sequesters normal tau into tangles of filaments and disassembles microtubules. Nat. Med. 2, 783-787.

Altar, C.A.; Vawter, M.P. and Ginsberg, S.D. (2009). Target identification for CNS diseases by transcriptional profiling. Neuropsychopharmacology 34, 18-54.

Alzheimer, A. (1907). "Über eine eigenartige Erkankung der Hirnrinde". Allg. Z. Psychiatr. 64, 146-148.

Alzheimer, A., Stelzmann, R.A., Schnitzlein, H.N., and Murtagh, F.R. (1995). An English translation of Alzheimer's 1907 paper, "Über eine eigenartige Erkankung der Hirnrinde". Clin Anat 8, 429-431.

Alzheimer's Association (2012). 2012 Alzheimer's disease facts and figures. Alzheimers Dement 8, 131-168.

American Psychiatric Association. (1995) Diagnostic and statistical manual of mental disorders, fourth edition. Primary care version DSM-IV-PC. Washington, DC, American Psychiatric Association 
Anders, S., and Huber, W. (2010). Differential expression analysis for sequence count data. Genome Biol. 11, R106.

Antonios, G., Saiepour, N., Bouter, Y., Richard, B.C., Paetau, A., Verkkoniemi-Ahola, A., Lannfelt, L., Ingelsson, M., Kovacs, G.G., and Pillot, T., et al. (2013). N-truncated Abeta starting with position four: early intraneuronal accumulation and rescue of toxicity using NT4X-167, a novel monoclonal antibody. Acta Neuropathol Commun 1, 56.

Aoki, M., Volkmann, I., Tjernberg, L.O., Winblad, B., and Bogdanovic, N. (2008). Amyloid beta-peptide levels in laser capture microdissected cornu ammonis 1 pyramidal neurons of Alzheimer's brain. Neuroreport 19, 1085-1089.

Aprahamian, I., Martinelli, J.E., Neri, A.L., and Yassuda, M.S. (2010). The accuracy of the Clock Drawing Test compared to that of standard screening tests for Alzheimer's disease: results from a study of Brazilian elderly with heterogeneous educational backgrounds. Int Psychogeriatr 22, 64-71.

Arendash, G.W., Gordon, M.N., Diamond, D.M., Austin, L.A., Hatcher, J.M., Jantzen, P., DiCarlo, G., Wilcock, D., and Morgan, D. (2001a). Behavioral assessment of Alzheimer's transgenic mice following long-term Abeta vaccination: task specificity and correlations between Abeta deposition and spatial memory. DNA Cell Biol 20, 737-744.

Arendash, G.W., King, D.L., Gordon, M.N., Morgan, D., Hatcher, J.M., Hope, C.E., and Diamond, D.M. (2001b). Progressive, age-related behavioral impairments in transgenic mice carrying both mutant amyloid precursor protein and presenilin-1 transgenes. Brain Res. 891, 42-53.

Arnaud, L., Robakis, N.K., and Figueiredo-Pereira, M.E. (2006). It may take inflammation, phosphorylation and ubiquitination to 'tangle' in Alzheimer's disease. Neurodegener Dis 3, 313-319.

Asai, M., Hattori, C., Szabo, B., Sasagawa, N., Maruyama, K., Tanuma, S.-I., and Ishiura, S. (2003). Putative function of ADAM9, ADAM10, and ADAM17 as APP alpha-secretase. Biochem Biophys Res Commun 301, 231-235.

Ashburner, M., Ball, C.A., Blake, J.A., Botstein, D., Butler, H., Cherry, J.M., Davis, A.P., Dolinski, K., Dwight, S.S., and Eppig, J.T., et al. (2000). Gene ontology: tool for the unification of biology. The Gene Ontology Consortium. Nat. Genet. 25, 25-29.

Baldan R., Lissandra C., and Pittenger, C. (2010). Cued and spatial learning in the water maze: equivalent learning in male and female mice. Neurosci Lett 483, 148-151.

Ballard, C., Gauthier, S., Corbett, A., Brayne, C., Aarsland, D., and Jones, E. (2011). Alzheimer's disease. The Lancet 377, 1019-1031.

Barbas, C.F., Burton, D.R., Scott, J.K., and Silverman, G.J. (2007). Quantitation of DNA and RNA. CSH Protoc 2007, pdb.ip47.

Bartus, R.T., Dean, R.L., Beer, B., and Lippa, A.S. (1982). The cholinergic hypothesis of geriatric memory dysfunction. Science $217,408-414$.

Bast, T., Zhang, W.-N., and Feldon, J. (2003). Dorsal hippocampus and classical fear conditioning to tone and context in rats: effects of local NMDA-receptor blockade and stimulation. Hippocampus 13, 657-675.

Bayer, T.A., Cappai, R., Masters, C.L., Beyreuther, K., and Multhaup, G. (1999). It all sticks together--the APP-related family of proteins and Alzheimer's disease. Mol Psychiatry 4, 524-528.

Bayer, T.A., and Wirths, O. (2008). Review on the APP/PS1KI mouse model: intraneuronal Abeta accumulation triggers axonopathy, neuron loss and working memory impairment. Genes Brain Behav 7 Suppl 1, 6-11. 
Bayer, T.A., and Wirths, O. (2010). Intracellular accumulation of amyloid-Beta - a predictor for synaptic dysfunction and neuron loss in Alzheimer's disease. Front Aging Neurosci 2, 8.

Bayer, T.A., and Wirths, O. (2011). Intraneuronal $A \beta$ as a trigger for neuron loss: can this be translated into human pathology? Biochem. Soc. Trans. 39, 857-861.

Bayer, T.A., and Wirths, O. (2014). Focusing the amyloid cascade hypothesis on $\mathrm{N}$ truncated Abeta peptides as drug targets against Alzheimer's disease. Acta Neuropathol.

Benilova, I., Karran, E., and DeStrooper, B. (2012). The toxic A $\beta$ oligomer and Alzheimer's disease: an emperor in need of clothes. Nat. Neurosci. 15, 349-357.

Bergamaschini, L., Canziani, S., Bottasso, B., Cugno, M., Braidotti, P., and Agostoni, A. (1999). Alzheimer's beta-amyloid peptides can activate the early components of complement classical pathway in a C1q-independent manner. Clin. Exp. Immunol. 115, 526-533.

Berglund, E.C., Kiialainen, A., and Syvänen, A.-C. (2011). Next-generation sequencing technologies and applications for human genetic history and forensics. Investig Genet 2, 23.

Bertram, L., Lill, C.M., and Tanzi, R.E. (2010). The genetics of Alzheimer disease: back to the future. Neuron 68, 270-281.

Bertram, L., and Tanzi, R.E. (2001). Dancing in the dark? The status of late-onset Alzheimer's disease genetics. J. Mol. Neurosci. 17, 127-136.

Bertram, L., and Tanzi, R.E. (2005). The genetic epidemiology of neurodegenerative disease. J. Clin. Invest. 115, 1449-1457.

Bettens, K., Sleegers, K., and van Broeckhoven, C. (2013). Genetic insights in Alzheimer's disease. Lancet Neurol 12, 92-104.

Bibl, M., Gallus, M., Welge, V., Esselmann, H., Wolf, S., Ruther, E., and Wiltfang, J. (2012). Cerebrospinal fluid amyloid-beta 2-42 is decreased in Alzheimer's, but not in frontotemporal dementia. J Neural Transm 119, 805-813.

Bickeboller, H., Campion, D., Brice, A., Amouyel, P., Hannequin, D., Didierjean, O., Penet, C., Martin, C., Perez-tur, J., and Michon, A., et al. (1997). Apolipoprotein E and Alzheimer disease: genotype-specific risks by age and sex. Am J Hum Genet 60, 439-446.

Bien, J., Jefferson, T., Causevic, M., Jumpertz, T., Munter, L., Multhaup, G., Weggen, S., Becker-Pauly, C., and Pietrzik, C.U. (2012). The metalloprotease meprin beta generates amino terminal-truncated amyloid beta peptide species. J Biol Chem 287, 33304-33313.

Billings, L.M., Oddo, S., Green, K.N., McGaugh, J.L., and LaFerla, F.M. (2005). Intraneuronal Abeta causes the onset of early Alzheimer's disease-related cognitive deficits in transgenic mice. Neuron 45, 675-688.

Blennow, K., deLeon, M.J., and Zetterberg, H. (2006). Alzheimer's disease. Lancet 368, 387-403.

Block, M.L., Zecca, L., and Hong, J.-S. (2007). Microglia-mediated neurotoxicity: uncovering the molecular mechanisms. Nat Rev Neurosci 8, 57-69.

Bossers, K., Wirz, K.T.S., Meerhoff, G.F., Essing, A.H.W., van Dongen, J.W., Houba, P., Kruse, C.G., Verhaagen, J., and Swaab, D.F. (2010). Concerted changes in transcripts in the prefrontal cortex precede neuropathology in Alzheimer's disease. Brain 133, 36993723.

Bottino, C. M., Castro, C.C., Gomes, Regina L E, Buchpiguel, C.A., Marchetti, R.L., and Neto, Mario R Louza (2002). Volumetric MRI measurements can differentiate Alzheimer's disease, mild cognitive impairment, and normal aging. Int Psychogeriatr 14, 59-72. 
Bouter, Y., Dietrich, K., Wittnam, J.L., Rezaei-Ghaleh, N., Pillot, T., Papot-Couturier, S., Lefebvre, T., Sprenger, F., Wirths, O., and Zweckstetter, M., et al. (2013). N-truncated amyloid $\beta$ (A $\beta$ ) 4-42 forms stable aggregates and induces acute and long-lasting behavioral deficits. Acta Neuropathol. 126, 189-205.

Bouter, Y., Kacprowski, T., Weissmann, R., Dietrich, K., Borgers, H., Brauß, A., Sperling, C., Wirths, O., Albrecht, M., and Jensen, L.R., et al. (2014). Deciphering the Molecular Profile of Plaques, Memory Decline and Neuron Loss in Two Mouse Models for Alzheimer's Disease by Deep Sequencing. Front. Aging Neurosci. 6.

Braak, H., and Braak, E. (1991). Neuropathological stageing of Alzheimer-related changes. Acta Neuropathol. 82, 239-259.

Breyhan, H., Wirths, O., Duan, K., Marcello, A., Rettig, J., and Bayer, T.A. (2009). APP/PS1KI bigenic mice develop early synaptic deficits and hippocampus atrophy. Acta Neuropathol. 117, 677-685.

Broadbent, N.J., Squire, L.R., and Clark, R.E. (2004). Spatial memory, recognition memory, and the hippocampus. Proc Natl Acad Sci USA 101, 14515-14520.

Brown, R.E., and Wong, A.A. (2007). The influence of visual ability on learning and memory performance in 13 strains of mice. Learn. Mem. 14, 134-144.

Bullock, R., and Dengiz, A. (2005). Cognitive performance in patients with Alzheimer's disease receiving cholinesterase inhibitors for up to 5 years. Int. J. Clin. Pract. 59, 817822.

Bullock, R., Touchon, J., Bergman, H., Gambina, G., He, Y., Rapatz, G., Nagel, J., and Lane, R. (2005). Rivastigmine and donepezil treatment in moderate to moderately-severe Alzheimer's disease over a 2-year period. Current Medical Research and Opinion.

Campion, D., Dumanchin, C., Hannequin, D., Dubois, B., Belliard, S., Puel, M., ThomasAnterion, C., Michon, A., Martin, C., and Charbonnier, F., et al. (1999). Early-onset autosomal dominant Alzheimer disease: prevalence, genetic heterogeneity, and mutation spectrum. Am. J. Hum. Genet. 65, 664-670.

Campos, A.C., Fogaça, M.V., Aguiar, D.C., and Guimarães, F.S. (2013). Animal models of anxiety disorders and stress. Rev Bras Psiquiatr 35 Suppl 2, S101-11.

Caroni, P. (1997). Overexpression of growth-associated proteins in the neurons of adult transgenic mice. J Neurosci Methods 71, 3-9.

Carrasquillo, M.M., Belbin, O., Hunter, T.A., Ma, L., Bisceglio, G.D., Zou, F., Crook, J.E., Pankratz, V.S., Dickson, D.W., and Graff-Radford, N.R., et al. (2010). Replication of CLU, CR1, and PICALM associations with alzheimer disease. Arch. Neurol. 67, 961-964.

Carrette, O., Demalte, I., Scherl, A., Yalkinoglu, O., Corthals, G., Burkhard, P., Hochstrasser, D.F., and Sanchez, J.-C. (2003). A panel of cerebrospinal fluid potential biomarkers for the diagnosis of Alzheimer's disease. Proteomics 3, 1486-1494.

Carroll, J.C., Rosario, E.R., Chang, L., Stanczyk, F.Z., Oddo, S., LaFerla, F.M., and Pike, C.J. (2007). Progesterone and estrogen regulate Alzheimer-like neuropathology in female 3xTg-AD mice. J Neurosci 27, 13357-13365.

Carson, J.A., and Turner, A.J. (2002). Beta-amyloid catabolism: roles for neprilysin (NEP) and other metallopeptidases? J Neurochem 81, 1-8.

Casas, C., Sergeant, N., Itier, J.-M., Blanchard, V., Wirths, O., van der Kolk, N., Vingtdeux, V., van de Steeg, E., Ret, G., and Canton, T., et al. (2004). Massive CA1/2 neuronal loss with intraneuronal and $\mathrm{N}$-terminal truncated Abeta42 accumulation in a novel Alzheimer transgenic model. Am. J. Pathol. 165, 1289-1300. 
Castellani, R.J., Nunomura, A., Lee, H.-g., Perry, G., and Smith, M.A. (2008). Phoshorylated tau: toxic, protective, or none of the above. J Alzheimers Dis 14, 377-383.

Castellano, J.M., Kim, J., Stewart, F.R., Jiang, H., DeMattos, R.B., Patterson, B.W., Fagan, A.M., Morris, J.C., Mawuenyega, K.G., and Cruchaga, C., et al. (2011). Human apoE Isoforms Differentially Regulate Brain Amyloid- Peptide Clearance. Science Translational Medicine 3, 89ra57.

Cataldo, A.M., Barnett, J.L., Berman, S.A., Li, J., Quarless, S., Bursztajn, S., Lippa, C., and Nixon, R.A. (1995). Gene expression and cellular content of cathepsin D in Alzheimer's disease brain: evidence for early up-regulation of the endosomal-lysosomal system. Neuron 14, 671-680.

Cavanaugh, S.E., Pippin, J.J., and Barnard, N.D. (2014). Animal models of Alzheimer disease: historical pitfalls and a path forward. ALTEX 31, 279-302.

Chen, G., Chen, K.S., Knox, J., Inglis, J., Bernard, A., Martin, S.J., Justice, A., McConlogue, L., Games, D., and Freedman, S.B., et al. (2000). A learning deficit related to age and beta-amyloid plaques in a mouse model of Alzheimer's disease. Nature 408, 975-979.

Chen, Y., Liang, Z., Blanchard, J., Dai, C.-L., Sun, S., Lee, M.H., Grundke-lqbal, I., lqbal, K., Liu, F., and Gong, C.-X. (2013). A non-transgenic mouse model (icv-STZ mouse) of Alzheimer's disease: similarities to and differences from the transgenic model (3xTg-AD mouse). Mol. Neurobiol. 47, 711-725.

Chen, Y., Tian, Z., Liang, Z., Sun, S., Dai, C.-L., Lee, M.H., LaFerla, F.M., Grundke-lqbal, I., Iqbal, K., and Liu, F., et al. (2012). Brain gene expression of a sporadic (icv-STZ Mouse) and a familial mouse model (3xTg-AD mouse) of Alzheimer's disease. PLoS ONE 7, e51432.

Cheng, L., Quek, C.Y.J., Sun, X., Bellingham, S.A., and Hill, A.F. (2013). The detection of microRNA associated with Alzheimer's disease in biological fluids using next-generation sequencing technologies. Front Genet 4, 150.

Chishti, M.A., Yang, D.S., Janus, C., Phinney, A.L., Horne, P., Pearson, J., Strome, R., Zuker, N., Loukides, J., and French, J., et al. (2001). Early-onset amyloid deposition and cognitive deficits in transgenic mice expressing a double mutant form of amyloid precursor protein 695. J. Biol. Chem. 276, 21562-21570.

Choi, J.W., Kang, S.M., Lee, Y., Hong, S.H., Sanek, N.A., Young, W.S., and Lee, H.J. (2013). MicroRNA profiling in the mouse hypothalamus reveals oxytocin-regulating microRNA. J. Neurochem. 126, 331-337.

Chow, V.W., Mattson, M.P., Wong, P.C., and Gleichmann, M. (2010). An overview of APP processing enzymes and products. Neuromolecular Med. 12, 1-12.

Christensen, D.Z., Bayer, T.A., and Wirths, O. (2010). Intracellular Aß triggers neuron loss in the cholinergic system of the APP/PS1KI mouse model of Alzheimer's disease. Neurobiol. Aging 31, 1153-1163.

Christensen, D.Z., Kraus, S.L., Flohr, A., Cotel, M.-C., Wirths, O., and Bayer, T.A. (2008). Transient intraneuronal A beta rather than extracellular plaque pathology correlates with neuron loss in the frontal cortex of APP/PS1KI mice. Acta Neuropathol. 116, 647-655.

Chui, D.H., Dobo, E., Makifuchi, T., Akiyama, H., Kawakatsu, S., Petit, A., Checler, F., Araki, W., Takahashi, K., and Tabira, T. (2001). Apoptotic neurons in Alzheimer's disease frequently show intracellular Abeta42 labeling. J Alzheimers Dis 3, 231-239.

Citron, M., Oltersdorf, T., Haass, C., McConlogue, L., Hung, A.Y., Seubert, P., VigoPelfrey, C., Lieberburg, I., and Selkoe, D.J. (1992). Mutation of the beta-amyloid precursor 
protein in familial Alzheimer's disease increases beta-protein production. Nature 360, $672-$ 674.

Citron, M., Teplow, D.B., and Selkoe, D.J. (1995). Generation of amyloid beta protein from its precursor is sequence specific. Neuron 14, 661-670.

Corcoran, K.A., Lu, Y., Turner, R.S., and Maren, S. (2002). Overexpression of hAPPswe impairs rewarded alternation and contextual fear conditioning in a transgenic mouse model of Alzheimer's disease. Learn Mem 9, 243-252.

Corder, E.H., Saunders, A.M., Strittmatter, W.J., Schmechel, D.E., Gaskell, P.C., Small, G.W., Roses, A.D., Haines, J.L., and Pericak-Vance, M.A. (1993). Gene dose of apolipoprotein $\mathrm{E}$ type 4 allele and the risk of Alzheimer's disease in late onset families. Science 261, 921-923.

Couillard-Despres, S., Winner, B., Schaubeck, S., Aigner, R., Vroemen, M., Weidner, N., Bogdahn, U., Winkler, J., Kuhn, H.-G., and Aigner, L. (2005). Doublecortin expression levels in adult brain reflect neurogenesis. Eur. J. Neurosci. 21, 1-14.

Coulson, E.J., Paliga, K., Beyreuther, K., and Masters, C.L. (2000). What the evolution of the amyloid protein precursor supergene family tells us about its function. Neurochem Int $36,175-184$.

Courtney, E., Kornfeld, S., Janitz, K., and Janitz, M. (2010). Transcriptome profiling in neurodegenerative disease. J. Neurosci. Methods 193, 189-202.

Cras, P., Smith, M.A., Richey, P.L., Siedlak, S.L., Mulvihill, P., and Perry, G. (1995). Extracellular neurofibrillary tangles reflect neuronal loss and provide further evidence of extensive protein cross-linking in Alzheimer disease. Acta Neuropathol. 89, 291-295.

Cruz, J.C., and Tsai, L.-H. (2004). Cdk5 deregulation in the pathogenesis of Alzheimer's disease. Trends Mol Med 10, 452-458.

D'Andrea, M.R., Nagele, R.G., Wang, H.Y., Peterson, P.A., and Lee, D.H. (2001). Evidence that neurones accumulating amyloid can undergo lysis to form amyloid plaques in Alzheimer's disease. Histopathology 38, 120-134.

Dawson, G.R., Seabrook, G.R., Zheng, H., Smith, D.W., Graham, S., O'Dowd, G., Bowery, B.J., Boyce, S., Trumbauer, M.E., and Chen, H.Y., et al. (1999). Age-related cognitive deficits, impaired long-term potentiation and reduction in synaptic marker density in mice lacking the $\beta$-amyloid precursor protein. Neuroscience 90, 1-13.

de la Monte, Suzanne M., and Wands, J.R. (2008). Alzheimer's Disease Is Type 3 Diabetes-Evidence Reviewed. J Diabetes Sci Technol 2, 1101-1113.

Deacon, Robert M J, and Rawlins, J Nicholas P (2006). T-maze alternation in the rodent. Nat Protoc 1, 7-12.

DeKosky, S.T., and Scheff, S.W. (1990). Synapse loss in frontal cortex biopsies in Alzheimer's disease: correlation with cognitive severity. Ann. Neurol. 27, 457-464.

Delacourte, A. (1990). General and dramatic glial reaction in Alzheimer brains. Neurology 40, 33-37.

Dember, W.N., and Fowler, H. (1958). Spontaneous alternation behavior. Psychological Bulletin 55, 412-428.

Dember, W.N., and Richman, C.L. (1989). Spontaneous Alternation Behavior (New York, NY: Springer New York).

Deng, H.-X., Chen, W., Hong, S.-T., Boycott, K.M., Gorrie, G.H., Siddique, N., Yang, Y., Fecto, F., Shi, Y., and Zhai, H., et al. (2011). Mutations in UBQLN2 cause dominant Xlinked juvenile and adult-onset ALS and ALS/dementia. Nature 477, 211-215. 
De-Paula, V.J., Radanovic, M., Diniz, B.S., and Forlenza, O.V. (2012). Alzheimer's disease. Subcell. Biochem. 65, 329-352.

Deutsche Alzheimer Gesellschaft. (2014) Die Häufigkeit von Demenzerkrankungen 2014.Selbsthilfe Demenz, Informationsblatt

Devi, L., and Ohno, M. (2010). Genetic reductions of beta-site amyloid precursor proteincleaving enzyme 1 and amyloid-beta ameliorate impairment of conditioned taste aversion memory in 5XFAD Alzheimer's disease model mice. Eur. J. Neurosci 31, 110-118.

Devi, L., and Ohno, M. (2013). Mechanisms that lessen benefits of $\beta$-secretase reduction in a mouse model of Alzheimer's disease. Transl Psychiatry 3, e284.

D'Hooge, R., and De Deyn, P P (2001). Applications of the Morris water maze in the study of learning and memory. Brain Res Brain Res Rev 36, 60-90.

Di Fede, G., Catania, M., Morbin, M., Rossi, G., Suardi, S., Mazzoleni, G., Merlin, M., Giovagnoli, A.R., Prioni, S., and Erbetta, A., et al. (2009). A recessive mutation in the APP gene with dominant-negative effect on amyloidogenesis. Science 323, 1473-1477.

Dickey, C.A., Loring, J.F., Montgomery, J., Gordon, M.N., Eastman, P.S., and Morgan, D. (2003). Selectively reduced expression of synaptic plasticity-related genes in amyloid precursor protein + presenilin-1 transgenic mice. J. Neurosci. 23, 5219-5226.

Ditaranto, K., Tekirian, T.L., and Yang, A.J. (2001). Lysosomal Membrane Damage in Soluble A $\beta$-Mediated Cell Death in Alzheimer's Disease. Neurobiology of Disease 8, 1931.

Dodart, J.C., Mathis, C., Saura, J., Bales, K.R., Paul, S.M., and Ungerer, A. (2000). Neuroanatomical abnormalities in behaviorally characterized APP(V717F) transgenic mice. Neurobiol Dis 7, 71-85.

Domenici, M.R., Paradisi, S., Sacchetti, B., Gaudi, S., Balduzzi, M., Bernardo, A., AjmoneCat, M.A., Minghetti, L., and Malchiodi-Albedi, F. (2002). The presence of astrocytes enhances beta amyloid-induced neurotoxicity in hippocampal cell cultures. J Physiol Paris 96, 313-316.

Drew, S.C., Masters, C.L., and Barnham, K.J. (2010). Alzheimer's Abeta peptides with disease-associated $\mathrm{N}$-terminal modifications: influence of isomerisation, truncation and mutation on Cu2+ coordination. PLoS ONE 5, e15875.

Duff, K., Eckman, C., Zehr, C., Yu, X., Prada, C.M., Perez-tur, J., Hutton, M., Buee, L., Harigaya, Y., and Yager, D., et al. (1996). Increased amyloid-beta42(43) in brains of mice expressing mutant presenilin 1. Nature 383, 710-713.

Duyckaerts, C., Delatour, B., and Potier, M.-C. (2009). Classification and basic pathology of Alzheimer disease. Acta Neuropathol 118, 5-36.

Duyckaerts, C., Potier, M.-C., and Delatour, B. (2008). Alzheimer disease models and human neuropathology: similarities and differences. Acta Neuropathol 115, 5-38.

Eckman, C.B., Mehta, N.D., Crook, R., Perez-tur, J., Prihar, G., Pfeiffer, E., Graff-Radford, N., Hinder, P., Yager, D., and Zenk, B., et al. (1997). A New Pathogenic Mutation in the APP Gene (I716V) Increases the Relative Proportion of A 42(43). Human Molecular Genetics 6, 2087-2089.

Eimer, W.A., and Vassar, R. (2013). Neuron loss in the 5XFAD mouse model of Alzheimer's disease correlates with intraneuronal $A \beta 42$ accumulation and Caspase- 3 activation. Mol Neurodegener 8, 2.

Elder, G.A., Gama Sosa, Miguel A, and Gasperi, R. de (2010). Transgenic mouse models of Alzheimer's disease. Mt. Sinai J. Med. 77, 69-81. 
Eminaga, S., Christodoulou, D.C., Vigneault, F., Church, G.M., and Seidman, J.G. (2013). Quantification of microRNA expression with next-generation sequencing. Curr Protoc Mol Biol Chapter 4, Unit 4.17.

Esch, F.S., Keim, P.S., Beattie, E.C., Blacher, R.W., Culwell, A.R., Oltersdorf, T., McClure, D., and Ward, P.J. (1990). Cleavage of amyloid beta peptide during constitutive processing of its precursor. Science 248, 1122-1124.

Esh, C., Patton, L., Kalback, W., Kokjohn, T.A., Lopez, J., Brune, D., Newell, A.J., Beach, T., Schenk, D., and Games, D., et al. (2005). Altered APP processing in PDAPP (Val717 - Phe) transgenic mice yields extended-length Abeta peptides. Biochemistry 44, 1380713819.

Espana, J., Gimenez-Llort, L., Valero, J., Minano, A., Rabano, A., Rodriguez-Alvarez, J., LaFerla, F.M., and Saura, C.A. (2010). Intraneuronal beta-amyloid accumulation in the amygdala enhances fear and anxiety in Alzheimer's disease transgenic mice. Biol Psychiatry 67, 513-521.

Farris, W., Mansourian, S., Chang, Y., Lindsley, L., Eckman, E.A., Frosch, M.P., Eckman, C.B., Tanzi, R.E., Selkoe, D.J., and Guenette, S. (2003). Insulin-degrading enzyme regulates the levels of insulin, amyloid beta-protein, and the beta-amyloid precursor protein intracellular domain in vivo. Proc. Natl. Acad. Sci. U.S.A. 100, 4162-4167.

Fernandez-Vizarra, P., Fernandez, A.P., Castro-Blanco, S., Serrano, J., Bentura, M.L., Martinez-Murillo, R., Martinez, A., and Rodrigo, J. (2004). Intra- and extracellular Abeta and PHF in clinically evaluated cases of Alzheimer's disease. Histol Histopathol 19, 823844.

Fiandaca, M.S., Mapstone, M.E., Cheema, A.K., and Federoff, H.J. (2014). The critical need for defining preclinical biomarkers in Alzheimer's disease. Alzheimers Dement 10, S196-212.

Filali, M., Lalonde, R., Theriault, P., Julien, C., Calon, F., and Planel, E. (2012). Cognitive and non-cognitive behaviors in the triple transgenic mouse model of Alzheimer's disease expressing mutated APP, PS1, and Mapt (3xTg-AD). Behav Brain Res 234, 334-342.

Franklin, K.B. and Paxinos, G. Paxinos and Franklin's (2012) The mouse brain in stereotaxic coordinates. 4th edition Academic Pr Inc

Fratiglioni, L., Paillard-Borg, S., and Winblad, B. (2004). An active and socially integrated lifestyle in late life might protect against dementia. The Lancet Neurology 3, 343-353.

Frost, J.L., Le, K.X., Cynis, H., Ekpo, E., Kleinschmidt, M., Palmour, R.M., Ervin, F.R., Snigdha, S., Cotman, C.W., and Saido, T.C., et al. (2013). Pyroglutamate-3 amyloid-beta deposition in the brains of humans, non-human primates, canines, and Alzheimer disease-like transgenic mouse models. Am J Pathol 183, 369-381.

Furukawa, K., Sopher, B.L., Rydel, R.E., Begley, J.G., Pham, D.G., Martin, G.M., Fox, M., and Mattson, M.P. (1996). Increased activity-regulating and neuroprotective efficacy of alpha-secretase-derived secreted amyloid precursor protein conferred by a C-terminal heparin-binding domain. J Neurochem 67, 1882-1896.

Galimberti, D., and Scarpini, E. (2012). Progress in Alzheimer's disease. J. Neurol. 259, 201-211.

Games, D., Adams, D., Alessandrini, R., Barbour, R., Berthelette, P., Blackwell, C., Carr, T., Clemens, J., Donaldson, T., and Gillespie, F. (1995). Alzheimer-type neuropathology in transgenic mice overexpressing V717F beta-amyloid precursor protein. Nature 373, 523527.

Games, D., Buttini, M., Kobayashi, D., Schenk, D., and Seubert, P. (2006). Mice as models: transgenic approaches and Alzheimer's disease. J. Alzheimers Dis 9, 133-149. 
Gatz, M., Reynolds, C.A., Fratiglioni, L., Johansson, B., Mortimer, J.A., Berg, S., Fiske, A., and Pedersen, N.L. (2006). Role of genes and environments for explaining Alzheimer disease. Arch. Gen. Psychiatry 63, 168-174.

George, A.J., Gordon, L., Beissbarth, T., Koukoulas, I., Holsinger, R.M., Perreau, V., Cappai, R., Tan, S.-S., Masters, C.L., and Scott, H.S., et al. (2010). A serial analysis of gene expression profile of the Alzheimer's disease Tg2576 mouse model. Neurotox Res 17, 360-379.

Gillette-Guyonnet, S., Nourhashemi, F., Andrieu, S., Glisezinski, I. de, Ousset, P.J., Riviere, D., Albarede, J.L., and Vellas, B. (2000). Weight loss in Alzheimer disease. Am. J. Clin. Nutr. 71, 637S-642S.

Ginzinger, D.G. (2002). Gene quantification using real-time quantitative PCR: an emerging technology hits the mainstream. Exp. Hematol. 30, 503-512.

Glabe, C.G., and Kayed, R. (2006). Common structure and toxic function of amyloid oligomers implies a common mechanism of pathogenesis. Neurology 66, S74-8.

Glaser, S., Schaft, J., Lubitz, S., Vintersten, K., van der Hoeven, F., Tufteland, K.R., Aasland, R., Anastassiadis, K., Ang, S.-L., and Stewart, A.F. (2006). Multiple epigenetic maintenance factors implicated by the loss of Mll2 in mouse development. Development 133, 1423-1432.

Goate, A., Chartier-Harlin, M.C., Mullan, M., Brown, J., Crawford, F., Fidani, L., Giuffra, L., Haynes, A., Irving, N., and James, L. (1991). Segregation of a missense mutation in the amyloid precursor protein gene with familial Alzheimer's disease. Nature 349, 704-706.

Goedert, M., and Jakes, R. (2005). Mutations causing neurodegenerative tauopathies. Biochim Biophys Acta 1739, 240-250.

Goedert, M., and Spillantini, M.G. (2006). A century of Alzheimer's disease. Science 314, 777-781.

Gomez-Isla, T., Hollister, R., West, H., Mui, S., Growdon, J.H., Petersen, R.C., Parisi, J.E., and Hyman, B.T. (1997). Neuronal loss correlates with but exceeds neurofibrillary tangles in Alzheimer's disease. Ann Neurol 41, 17-24.

Gomez-Isla, T., Price, J.L., McKeel, D W Jr, Morris, J.C., Growdon, J.H., and Hyman, B.T. (1996). Profound loss of layer II entorhinal cortex neurons occurs in very mild Alzheimer's disease. J Neurosci 16, 4491-4500.

Götz, J., and Ittner, L.M. (2008). Animal models of Alzheimer's disease and frontotemporal dementia. Nat. Rev. Neurosci. 9, 532-544.

Gouras, G.K., Tampellini, D., Takahashi, R.H., and Capetillo-Zarate, E. (2010). Intraneuronal beta-amyloid accumulation and synapse pathology in Alzheimer's disease. Acta Neuropathol. 119, 523-541.

Gouras, G.K., Tsai, J., Naslund, J., Vincent, B., Edgar, M., Checler, F., Greenfield, J.P., Haroutunian, V., Buxbaum, J.D., and Xu, H., et al. (2000). Intraneuronal Abeta42 accumulation in human brain. Am J Pathol 156, 15-20.

Grabowski, T.J., Cho, H.S., Vonsattel, J.P., Rebeck, G.W., and Greenberg, S.M. (2001). Novel amyloid precursor protein mutation in an lowa family with dementia and severe cerebral amyloid angiopathy. Ann Neurol 49, 697-705.

Gu, Y., Nieves, J.W., Stern, Y., Luchsinger, J.A., and Scarmeas, N. (2010). Food combination and Alzheimer disease risk: a protective diet. Arch. Neurol. 67, 699-706.

Guérin, O., Andrieu, S., Schneider, S.M., Milano, M., Boulahssass, R., Brocker, P., and Vellas, B. (2005). Different modes of weight loss in Alzheimer disease: a prospective study of 395 patients. Am. J. Clin. Nutr. 82, 435-441. 
Guerreiro, R., Wojtas, A., Bras, J., Carrasquillo, M., Rogaeva, E., Majounie, E., Cruchaga, C., Sassi, C., Kauwe, John S K, and Younkin, S., et al. (2013). TREM2 variants in Alzheimer's disease. N. Engl. J. Med. 368, 117-127.

Guntert, A., Dobeli, H., and Bohrmann, B. (2006). High sensitivity analysis of amyloid-beta peptide composition in amyloid deposits from human and PS2APP mouse brain. Neuroscience 143, 461-475.

Gyure, K.A., Durham, R., Stewart, W.F., Smialek, J.E., and Troncoso, J.C. (2001). Intraneuronal abeta-amyloid precedes development of amyloid plaques in Down syndrome. Arch Pathol Lab Med 125, 489-492.

Haass, C., and Selkoe, D.J. (2007). Soluble protein oligomers in neurodegeneration: lessons from the Alzheimer's amyloid beta-peptide. Nat. Rev. Mol. Cell Biol. 8, 101-112.

Hall, C.B., Lipton, R.B., Sliwinski, M., Katz, M.J., Derby, C.A., and Verghese, J. (2009). Cognitive activities delay onset of memory decline in persons who develop dementia. Neurology 73, 356-361.

Hamann, S., Monarch, E.S., and Goldstein, F.C. (2002). Impaired fear conditioning in Alzheimer's disease. Neuropsychologia 40, 1187-1195.

Handel, A.E., Disanto, G., and Ramagopalan, S.V. (2013). Next-generation sequencing in understanding complex neurological disease. Expert Rev Neurother 13, 215-227.

Hardy, J., and Allsop, D. (1991). Amyloid deposition as the central event in the aetiology of Alzheimer's disease. Trends Pharmacol. Sci. 12, 383-388.

Harigaya, Y., Saido, T.C., Eckman, C.B., Prada, C.M., Shoji, M., and Younkin, S.G. (2000). Amyloid beta protein starting pyroglutamate at position 3 is a major component of the amyloid deposits in the Alzheimer's disease brain. Biochem Biophys Res Commun 276, $422-427$.

Harold, D., Abraham, R., Hollingworth, P., Sims, R., Gerrish, A., Hamshere, M.L., Pahwa, J.S., Moskvina, V., Dowzell, K., and Williams, A., et al. (2009). Genome-wide association study identifies variants at CLU and PICALM associated with Alzheimer's disease. Nat. Genet. 41, 1088-1093.

Hartig, W., Goldhammer, S., Bauer, U., Wegner, F., Wirths, O., Bayer, T.A., and Grosche, J. (2010). Concomitant detection of beta-amyloid peptides with $\mathrm{N}$-terminal truncation and different $\mathrm{C}$-terminal endings in cortical plaques from cases with Alzheimer's disease, senile monkeys and triple transgenic mice. J Chem Neuroanat 40, 82-92.

Hau, J., and Schapiro, S.J. (2002). Handbook of Laboratory Animal Science, Second Edition: Essential Principles and Practices (Taylor \& Francis).

Haughey, N.J., Liu, D., Nath, A., Borchard, A.C., and Mattson, M.P. (2002a). Disruption of neurogenesis in the subventricular zone of adult mice, and in human cortical neuronal precursor cells in culture, by amyloid beta-peptide: implications for the pathogenesis of Alzheimer's disease. Neuromolecular Med. 1, 125-135.

Haughey, N.J., Nath, A., Chan, S.L., Borchard, A.C., Rao, M.S., and Mattson, M.P. (2002b). Disruption of neurogenesis by amyloid beta-peptide, and perturbed neural progenitor cell homeostasis, in models of Alzheimer's disease. J. Neurochem. 83, 15091524.

Haupt, C., Leppert, J., Rönicke, R., Meinhardt, J., Yadav, J.K., Ramachandran, R., Ohlenschläger, O., Reymann, K.G., Görlach, M., and Fändrich, M. (2012). Structural basis of $\beta$-amyloid-dependent synaptic dysfunctions. Angew. Chem. Int. Ed. Engl. 51, 15761579. 
He, W., and Barrow, C.J. (1999). The A beta 3-pyroglutamyl and 11-pyroglutamyl peptides found in senile plaque have greater beta-sheet forming and aggregation propensities in vitro than full-length A beta. Biochemistry 38, 10871-10877.

Heber, S., Herms, J., Gajic, V., Hainfellner, J., Aguzzi, A., Rulicke, T., Kretzschmar, H. von, Koch, C. von, Sisodia, S., and Tremml, P., et al. (2000). Mice with combined gene knock-outs reveal essential and partially redundant functions of amyloid precursor protein family members. J Neurosci 20, 7951-7963.

Heneka, M.T., O'Banion, M.K., Terwel, D., and Kummer, M.P. (2010). Neuroinflammatory processes in Alzheimer's disease. J Neural Transm 117, 919-947.

Hirokawa, N., Niwa, S., and Tanaka, Y. (2010). Molecular motors in neurons: transport mechanisms and roles in brain function, development, and disease. Neuron 68, 610-638.

Hirokawa, N., Noda, Y., Tanaka, Y., and Niwa, S. (2009). Kinesin superfamily motor proteins and intracellular transport. Nat. Rev. Mol. Cell Biol. 10, 682-696.

Hoefer, M., Allison, S.C., Schauer, G.F., Neuhaus, J.M., Hall, J., Dang, J.N., Weiner, M.W., Miller, B.L., and Rosen, H.J. (2008). Fear conditioning in frontotemporal lobar degeneration and Alzheimer's disease. Brain 131, 1646-1657.

Holcomb, L., Gordon, M.N., McGowan, E., Yu, X., Benkovic, S., Jantzen, P., Wright, K., Saad, I., Mueller, R., and Morgan, D., et al. (1998). Accelerated Alzheimer-type phenotype in transgenic mice carrying both mutant amyloid precursor protein and presenilin 1 transgenes. Nat Med 4, 97-100.

Hollingworth, P., Harold, D., Sims, R., Gerrish, A., Lambert, J.-C., Carrasquillo, M.M., Abraham, R., Hamshere, M.L., Pahwa, J.S., and Moskvina, V., et al. (2011). Common variants at ABCA7, MS4A6A/MS4A4E, EPHA1, CD33 and CD2AP are associated with Alzheimer's disease. Nat. Genet. 43, 429-435.

Holmes, C., Boche, D., Wilkinson, D., Yadegarfar, G., Hopkins, V., Bayer, A., Jones, R.W., Bullock, R., Love, S., and Neal, J.W., et al. (2008). Long-term effects of Abeta42 immunisation in Alzheimer's disease: follow-up of a randomised, placebo-controlled phase I trial. Lancet 372, 216-223.

Holtzman, D.M., Morris, J.C., and Goate, A.M. (2011). Alzheimer's disease: the challenge of the second century. Sci Transl Med 3, 77sr1.

Hook, V., Toneff, T., Bogyo, M., Greenbaum, D., Medzihradszky, K.F., Neveu, J., Lane, W., Hook, G., and Reisine, T. (2005). Inhibition of cathepsin B reduces beta-amyloid production in regulated secretory vesicles of neuronal chromaffin cells: evidence for cathepsin B as a candidate beta-secretase of Alzheimer's disease. Biol Chem 386, 931940.

Hook, V.Y., Kindy, M., Reinheckel, T., Peters, C., and Hook, G. (2009). Genetic cathepsin $B$ deficiency reduces beta-amyloid in transgenic mice expressing human wild-type amyloid precursor protein. Biochem. Biophys. Res. Commun. 386, 284-288.

Hou, L., Kang, I., Marchant, R.E., and Zagorski, M.G. (2002). Methionine 35 oxidation reduces fibril assembly of the amyloid abeta-(1-42) peptide of Alzheimer's disease. J Biol Chem 277, 40173-40176.

Howell, S., Nalbantoglu, J., and Crine, P. (1995). Neutral endopeptidase can hydrolyze beta-amyloid(1-40) but shows no effect on beta-amyloid precursor protein metabolism. Peptides 16, 647-652.

Hsiao, K., Chapman, P., Nilsen, S., Eckman, C., Harigaya, Y., Younkin, S., Yang, F., and Cole, G. (1996). Correlative memory deficits, Abeta elevation, and amyloid plaques in transgenic mice. Science 274, 99-102. 
Hsieh, C.L., Koike, M., Spusta, S.C., Niemi, E.C., Yenari, M., Nakamura, M.C., and Seaman, W.E. (2009). A role for TREM2 ligands in the phagocytosis of apoptotic neuronal cells by microglia. J. Neurochem. 109, 1144-1156.

Hussain, I., Powell, D., Howlett, D.R., Tew, D.G., Meek, T.D., Chapman, C., Gloger, I.S., Murphy, K.E., Southan, C.D., and Ryan, D.M., et al. (1999). Identification of a novel aspartic protease (Asp 2) as beta-secretase. Mol Cell Neurosci 14, 419-427.

Hutton, M., Lendon, C.L., Rizzu, P., Baker, M., Froelich, S., Houlden, H., Pickering-Brown, S., Chakraverty, S., Isaacs, A., and Grover, A., et al. (1998). Association of missense and 5 '-splice-site mutations in tau with the inherited dementia FTDP-17. Nature 393, 702-705.

Ingelsson, M., Fukumoto, H., Newell, K.L., Growdon, J.H., Hedley-Whyte, E.T., Frosch, M.P., Albert, M.S., Hyman, B.T., and Irizarry, M.C. (2004). Early Abeta accumulation and progressive synaptic loss, gliosis, and tangle formation in AD brain. Neurology 62, 925931.

lqbal, K., Alonso, Alejandra del C, Chen, S., Chohan, M.O., El-Akkad, E., Gong, C.-X., Khatoon, S., Li, B., Liu, F., and Rahman, A., et al. (2005a). Tau pathology in Alzheimer disease and other tauopathies. Biochim. Biophys. Acta 1739, 198-210.

Iqbal, K., Flory, M., Khatoon, S., Soininen, H., Pirttila, T., Lehtovirta, M., Alafuzoff, I., Blennow, K., Andreasen, N., and Vanmechelen, E., et al. (2005b). Subgroups of Alzheimer's disease based on cerebrospinal fluid molecular markers. Ann Neurol 58, 748757.

Iqbal, K., Bolognin, S., Wang, X., Basurto-Islas, G., Blanchard, J., and Tung, Y.C. (2013). Animal models of the sporadic form of Alzheimer's disease: focus on the disease and not just the lesions. J. Alzheimers Dis. 37, 469-474.

Irizarry, M.C., Soriano, F., McNamara, M., Page, K.J., Schenk, D., Games, D., and Hyman, B.T. (1997). Abeta deposition is associated with neuropil changes, but not with overt neuronal loss in the human amyloid precursor protein V717F (PDAPP) transgenic mouse. J Neurosci 17, 7053-7059.

Itagaki, S., McGeer, P., Akiyama, H., Zhu, S., and Selkoe, D. (1989). Relationship of microglia and astrocytes to amyloid deposits of Alzheimer disease. Journal of Neuroimmunology 24, 173-182.

Iwata, N., Tsubuki, S., Takaki, Y., Shirotani, K., Lu, B., Gerard, N.P., Gerard, C., Hama, E., Lee, H.J., and Saido, T.C. (2001). Metabolic regulation of brain Abeta by neprilysin. Science 292, 1550-1552.

Iwatsubo, T., Odaka, A., Suzuki, N., Mizusawa, H., Nukina, N., and Ihara, Y. (1994). Visualization of A beta 42(43) and A beta 40 in senile plaques with end-specific A beta monoclonals: evidence that an initially deposited species is A beta 42(43). Neuron 13, $45-$ 53.

Jack, C.R., Albert, M.S., Knopman, D.S., McKhann, G.M., Sperling, R.A., Carrillo, M.C., Thies, B., and Phelps, C.H. (2011). Introduction to the recommendations from the National Institute on Aging-Alzheimer's Association workgroups on diagnostic guidelines for Alzheimer's disease. Alzheimers Dement 7, 257-262.

Jack, C R Jr, Shiung, M.M., Weigand, S.D., O'Brien, P.C., Gunter, J.L., Boeve, B.F., Knopman, D.S., Smith, G.E., Ivnik, R.J., and Tangalos, E.G., et al. (2005). Brain atrophy rates predict subsequent clinical conversion in normal elderly and amnestic $\mathrm{MCl}$. Neurology 65, 1227-1231.

Jaffrey, S.R., Benfenati, F., Snowman, A.M., Czernik, A.J., and Snyder, S.H. (2002). Neuronal nitric-oxide synthase localization mediated by a ternary complex with synapsin and CAPON. Proc. Natl. Acad. Sci. U.S.A. 99, 3199-3204. 
Jagust, W. (2006). Positron emission tomography and magnetic resonance imaging in the diagnosis and prediction of dementia. Alzheimers Dement 2, 36-42.

Jahn, H., Wittke, S., Zürbig, P., Raedler, T.J., Arlt, S., Kellmann, M., Mullen, W., Eichenlaub, M., Mischak, H., and Wiedemann, K. (2011). Peptide fingerprinting of Alzheimer's disease in cerebrospinal fluid: identification and prospective evaluation of new synaptic biomarkers. PLoS ONE 6, e26540.

Jan, A., Gokce, O., Luthi-Carter, R., and Lashuel, H.A. (2008). The ratio of monomeric to aggregated forms of Abeta40 and Abeta42 is an important determinant of amyloid-beta aggregation, fibrillogenesis, and toxicity. J. Biol. Chem. 283, 28176-28189.

Jankowsky, J.L., Younkin, L.H., Gonzales, V., Fadale, D.J., Slunt, H.H., Lester, H.A., Younkin, S.G., and Borchelt, D.R. (2007). Rodent A beta modulates the solubility and distribution of amyloid deposits in transgenic mice. J Biol Chem 282, 22707-22720.

Jarrett, J.T., Berger, E.P., and Lansbury, P.T. (1993). The carboxy terminus of the .beta. amyloid protein is critical for the seeding of amyloid formation: Implications for the pathogenesis of Alzheimer's disease. Biochemistry 32, 4693-4697.

Jawhar, S., Trawicka, A., Jenneckens, C., Bayer, T.A., and Wirths, O. (2010). Motor deficits, neuron loss, and reduced anxiety coinciding with axonal degeneration and intraneuronal Abeta aggregation in the 5XFAD mouse model of Alzheimer's disease. Neurobiol. Aging.

Jawhar, S., Wirths, O., and Bayer, T.A. (2011). Pyroglutamate amyloid- $\beta$ (A $\beta$ ): a hatchet man in Alzheimer disease. J. Biol. Chem. 286, 38825-38832.

Jin, K., Peel, A.L., Mao, X.O., Xie, L., Cottrell, B.A., Henshall, D.C., and Greenberg, D.A. (2004). Increased hippocampal neurogenesis in Alzheimer's disease. Proc. Natl. Acad. Sci. U.S.A. 101, 343-347.

Jin, S.C., Pastor, P., Cooper, B., Cervantes, S., Benitez, B.A., Razquin, C., Goate, A., and Cruchaga, C. (2012). Pooled-DNA sequencing identifies novel causative variants in PSEN1, GRN and MAPT in a clinical early-onset and familial Alzheimer's disease lberoAmerican cohort. Alzheimers Res Ther 4, 34.

Johnston, J.A., Liu, W.W., Todd, S.A., Coulson, D.T., Murphy, S., Irvine, G.B., and Passmore, A.P. (2005). Expression and activity of beta-site amyloid precursor protein cleaving enzyme in Alzheimer's disease. Biochem Soc Trans 33, 1096-1100.

Jonghe, C. de, Esselens, C., Kumar-Singh, S., Craessaerts, K., Serneels, S., Checler, F., Annaert, W., van Broeckhoven, C., and Strooper, B. de (2001). Pathogenic APP mutations near the gamma-secretase cleavage site differentially affect Abeta secretion and APP C-terminal fragment stability. Hum Mol Genet 10, 1665-1671.

Jonsson, T., Stefansson, H., Steinberg, S., Jonsdottir, I., Jonsson, P.V., Snaedal, J., Bjornsson, S., Huttenlocher, J., Levey, A.I., and Lah, J.J., et al. (2013). Variant of TREM2 associated with the risk of Alzheimer's disease. N. Engl. J. Med. 368, 107-116.

Kalback, W., Watson, M.D., Kokjohn, T.A., Kuo, Y.-M., Weiss, N., Luehrs, D.C., Lopez, J., Brune, D., Sisodia, S.S., and Staufenbiel, M., et al. (2002). APP transgenic mice Tg2576 accumulate Abeta peptides that are distinct from the chemically modified and insoluble peptides deposited in Alzheimer's disease senile plaques. Biochemistry 41, 922-928.

Kalinin, S., Richardson, J.C., and Feinstein, D.L. (2009). A PPARdelta agonist reduces amyloid burden and brain inflammation in a transgenic mouse model of Alzheimer's disease. Curr Alzheimer Res 6, 431-437.

Kaminski, W.E., Orsó, E., Diederich, W., Klucken, J., Drobnik, W., and Schmitz, G. (2000). Identification of a novel human sterol-sensitive ATP-binding cassette transporter (ABCA7). Biochem. Biophys. Res. Commun. 273, 532-538. 
Kanekiyo, T., Cirrito, J.R., Liu, C.-C., Shinohara, M., Li, J., Schuler, D.R., Shinohara, M., Holtzman, D.M., and Bu, G. (2013). Neuronal clearance of amyloid- $\beta$ by endocytic receptor LRP1. J. Neurosci. 33, 19276-19283.

Karl, T., Pabst, R., and Hörsten, S. von (2003). Behavioral phenotyping of mice in pharmacological and toxicological research. Exp. Toxicol. Pathol. 55, 69-83.

Kasuga, K., Shimohata, T., Nishimura, A., Shiga, A., Mizuguchi, T., Tokunaga, J., Ohno, T., Miyashita, A., Kuwano, R., and Matsumoto, N., et al. (2009). Identification of independent APP locus duplication in Japanese patients with early-onset Alzheimer disease. J. Neurol. Neurosurg. Psychiatr. 80, 1050-1052.

Kayed, R., Head, E., Thompson, J.L., Mclntire, T.M., Milton, S.C., Cotman, C.W., and Glabe, C.G. (2003). Common structure of soluble amyloid oligomers implies common mechanism of pathogenesis. Science 300, 486-489.

Kerimoglu, C., Agis-Balboa, R.C., Kranz, A., Stilling, R., Bahari-Javan, S., BenitoGaragorri, E., Halder, R., Burkhardt, S., Stewart, A.F., and Fischer, A. (2013). Histonemethyltransferase MLL2 (KMT2B) is required for memory formation in mice. J. Neurosci. 33, 3452-3464.

Kielar, D., Kaminski, W.E., Liebisch, G., Piehler, A., Wenzel, J.J., Möhle, C., Heimerl, S., Langmann, T., Friedrich, S.O., and Böttcher, A., et al. (2003). Adenosine triphosphate binding cassette $(A B C)$ transporters are expressed and regulated during terminal keratinocyte differentiation: a potential role for ABCA7 in epidermal lipid reorganization. J. Invest. Dermatol. 121, 465-474.

Kim, J., Basak, J.M., and Holtzman, D.M. (2009). The role of apolipoprotein E in Alzheimer's disease. Neuron 63, 287-303.

Kishimoto, Y., Higashihara, E., Fukuta, A., Nagao, A., and Kirino, Y. (2013). Early impairment in a water-finding test in a longitudinal study of the Tg2576 mouse model of Alzheimer's disease. Brain Res. 1491, 117-126.

Kivipelto, M. (2001). Midlife vascular risk factors and Alzheimer's disease in later life: longitudinal, population based study. BMJ 322, 1447-1451.

Kivipelto, M., Ngandu, T., Fratiglioni, L., Viitanen, M., Kåreholt, I., Winblad, B., Helkala, E.L., Tuomilehto, J., Soininen, H., and Nissinen, A. (2005). Obesity and vascular risk factors at midlife and the risk of dementia and Alzheimer disease. Arch. Neurol. 62, 1556-1560.

Knafo, S., Venero, C., Merino-Serrais, P., Fernaud-Espinosa, I., Gonzalez-Soriano, J., Ferrer, I., Santpere, G., and DeFelipe, J. (2009). Morphological alterations to neurons of the amygdala and impaired fear conditioning in a transgenic mouse model of Alzheimer's disease. J. Pathol. 219, 41-51.

Ko, H.S., Uehara, T., Tsuruma, K., and Nomura, Y. (2004). Ubiquilin interacts with ubiquitylated proteins and proteasome through its ubiquitin-associated and ubiquitin-like domains. Ubqln2. FEBS Lett. 566, 110-114.

Komada, M., Takao, K., and Miyakawa, T. (2008). Elevated plus maze for mice. J Vis Exp. 22

Kondo, M., Takei, Y., and Hirokawa, N. (2012). Motor protein KIF1A is essential for hippocampal synaptogenesis and learning enhancement in an enriched environment. Neuron 73, 743-757.

Korenberg, J.R., Pulst, S.M., Neve, R.L., and West, R. (1989). The Alzheimer amyloid precursor protein maps to human chromosome 21 bands q21.105-q21.05. Genomics 5, 124-127.

Krause, D.L., and Müller, N. (2010). Neuroinflammation, microglia and implications for anti-inflammatory treatment in Alzheimer's disease. Int J Alzheimers Dis 2010. 
Krumlauf, R. (1994). Analysis of gene expression by northern blot. Mol Biotechnol 2, 227242.

Kuhn, P.-H., Wang, H., Dislich, B., Colombo, A., Zeitschel, U., Ellwart, J.W., Kremmer, E., Rossner, S., and Lichtenthaler, S.F. (2010). ADAM10 is the physiologically relevant, constitutive alpha-secretase of the amyloid precursor protein in primary neurons. EMBO $\mathrm{J}$ 29, 3020-3032.

Kumar, S., Rezaei-Ghaleh, N., Terwel, D., Thal, D.R., Richard, M., Hoch, M., Mc Donald, Jessica M, Wüllner, U., Glebov, K., and Heneka, M.T., et al. (2011). Extracellular phosphorylation of the amyloid $\beta$-peptide promotes formation of toxic aggregates during the pathogenesis of Alzheimer's disease. EMBO J. 30, 2255-2265.

Kummer, M.P., and Heneka, M.T. (2014). Truncated and modified amyloid-beta species. Alzheimers Res Ther 6, 28.

LaFerla, F.M., Tinkle, B.T., Bieberich, C.J., Haudenschild, C.C., and Jay, G. (1995). The Alzheimer's A beta peptide induces neurodegeneration and apoptotic cell death in transgenic mice. Nat Genet 9, 21-30.

Lalonde, R. (2002). The neurobiological basis of spontaneous alternation. Neuroscience \& Biobehavioral Reviews 26, 91-104.

Lalonde, R., Dumont, M., Staufenbiel, M., Sturchler-Pierrat, C., and Strazielle, C. (2002). Spatial learning, exploration, anxiety, and motor coordination in female APP23 transgenic mice with the Swedish mutation. Brain Res 956, 36-44.

Lalonde, R., and Strazielle, C. (2007). Brain regions and genes affecting postural control. Prog Neurobiol 81, 45-60.

Lalonde, R., Fukuchi, K.-I., and Strazielle, C. (2012). Neurologic and motor dysfunctions in APP transgenic mice. Reviews in the neurosciences 23, 363-379.

Lambert, J.-C., Heath, S., Even, G., Campion, D., Sleegers, K., Hiltunen, M., Combarros, O., Zelenika, D., Bullido, M.J., and Tavernier, B., et al. (2009). Genome-wide association study identifies variants at CLU and CR1 associated with Alzheimer's disease. Nat. Genet. 41, 1094-1099.

Lambert, J.-C., Ibrahim-Verbaas, C.A., Harold, D., Naj, A.C., Sims, R., Bellenguez, C., Jun, G., Destefano, A.L., Bis, J.C., and Beecham, G.W., et al. (2013). Meta-analysis of 74,046 individuals identifies 11 new susceptibility loci for Alzheimer's disease. Nat. Genet. $45,1452-1458$.

Lang, F., Strutz-Seebohm, N., Seebohm, G., and Lang, U.E. (2010). Significance of SGK1 in the regulation of neuronal function. J. Physiol. (Lond.) 588, 3349-3354.

Larson, M.E., and Lesné, S.E. (2012). Soluble $A \beta$ oligomer production and toxicity. J. Neurochem. 120 Suppl 1, 125-139.

LeDoux, J. (2003). The emotional brain, fear, and the amygdala. Cell Mol Neurobiol 23, 727-738.

Leibson, C.L., Rocca, W.A., Hanson, V.A., Cha, R., Kokmen, E., O'Brien, P.C., and Palumbo, P.J. (1997). Risk of dementia among persons with diabetes mellitus: a population-based cohort study. Am. J. Epidemiol. 145, 301-308.

Leskovjan, A.C., Kretlow, A., Lanzirotti, A., Barrea, R., Vogt, S., and Miller, L.M. (2011). Increased brain iron coincides with early plaque formation in a mouse model of Alzheimer's disease. Neuroimage 55, 32-38.

Lesne, S., Kotilinek, L., and Ashe, K.H. (2008). Plaque-bearing mice with reduced levels of oligomeric amyloid-beta assemblies have intact memory function. Neuroscience 151, 745-749. 
Levi, A., Ferri, G.-L., Watson, E., Possenti, R., and Salton, S.R.J. (2004). Processing, distribution, and function of VGF, a neuronal and endocrine peptide precursor. Cell. Mol. Neurobiol. 24, 517-533.

Lewis, H., Beher, D., Cookson, N., Oakley, A., Piggott, M., Morris, C.M., Jaros, E., Perry, R., Ince, P., and Kenny, R.A., et al. (2006). Quantification of Alzheimer pathology in ageing and dementia: age-related accumulation of amyloid-beta(42) peptide in vascular dementia. Neuropathol. Appl. Neurobiol. 32, 103-118.

Li, C., Zhao, R., Gao, K., Wei, Z., Yin, M.Y., Lau, L.T., Chui, D., and Hoi Yu, Albert Cheung (2011). Astrocytes: implications for neuroinflammatory pathogenesis of Alzheimer's disease. Curr Alzheimer Res 8, 67-80.

Li, S., Hong, S., Shepardson, N.E., Walsh, D.M., Shankar, G.M., and Selkoe, D. (2009). Soluble oligomers of amyloid Beta protein facilitate hippocampal long-term depression by disrupting neuronal glutamate uptake. Neuron 62, 788-801.

$\mathrm{Li}, \mathrm{X}$., and Buxbaum, J.N. (2011). Transthyretin and the brain re-visited: is neuronal synthesis of transthyretin protective in Alzheimer's disease? Mol Neurodegener 6, 79.

Liao, M.-C., Ahmed, M., Smith, S.O., and Van Nostrand, William E (2009). Degradation of amyloid beta protein by purified myelin basic protein. J Biol Chem 284, 28917-28925.

Lidström, A.M., Bogdanovic, N., Hesse, C., Volkman, I., Davidsson, P., and Blennow, K. (1998). Clusterin (apolipoprotein J) protein levels are increased in hippocampus and in frontal cortex in Alzheimer's disease. Exp. Neurol. 154, 511-521.

Liu, K., Doms, R.W., and Lee, V.M.-Y. (2002). Glu11 site cleavage and N-terminally truncated A beta production upon BACE overexpression. Biochemistry 41, 3128-3136.

Liu, R.-Q., Zhou, Q.-H., Ji, S.-R., Zhou, Q., Du Feng, Wu, Y., and Sui, S.-F. (2010). Membrane localization of beta-amyloid 1-42 in lysosomes: a possible mechanism for lysosome labilization. J Biol Chem 285, 19986-19996.

Loeffler, D.A., Camp, D.M., and Conant, S.B. (2006). Complement activation in the Parkinson's disease substantia nigra: an immunocytochemical study. J Neuroinflammation 3, 29.

Lovell, M.A., Robertson, J.D., Teesdale, W.J., Campbell, J.L., and Markesbery, W.R. (1998). Copper, iron and zinc in Alzheimer's disease senile plaques. J. Neurol. Sci. 158, 47-52.

Luong, T.N., Carlisle, H.J., Southwell, A., and Patterson, P.H. (2011). Assessment of motor balance and coordination in mice using the balance beam. $J$ Vis Exp.

Lyketsos, C.G., Lopez, O., Jones, B., Fitzpatrick, A.L., Breitner, J., and DeKosky, S. (2002). Prevalence of neuropsychiatric symptoms in dementia and mild cognitive impairment: results from the cardiovascular health study. JAMA 288, 1475-1483.

Magalhães, J.P. de, Finch, C.E., and Janssens, G. (2010). Next-generation sequencing in aging research: emerging applications, problems, pitfalls and possible solutions. Ageing Res. Rev. 9, 315-323.

Hullmann M. (2012). Evaluation of the effects of inhaled nanoparticles on the central nervous system of mice. Doktorarbeit, Heinrich Heine Universität Düsseldorf.

Malone, J.H., and Oliver, B. (2011). Microarrays, deep sequencing and the true measure of the transcriptome. BMC Biol. 9, 34.

Martinelli, J.E., Cecato, J.F., Bartholomeu, D., and Montiel, J.M. (2014). Comparison of the diagnostic accuracy of neuropsychological tests in differentiating Alzheimer's disease from mild cognitive impairment: can the montreal cognitive assessment be better than the cambridge cognitive examination? Dement Geriatr Cogn Dis Extra 4, 113-121. 
Masters, C.L., Simms, G., Weinman, N.A., Multhaup, G., McDonald, B.L., and Beyreuther, K. (1985). Amyloid plaque core protein in Alzheimer disease and Down syndrome. Proc. Natl. Acad. Sci. U.S.A. 82, 4245-4249.

Mattson, M.P. (1997). Cellular actions of beta-amyloid precursor protein and its soluble and fibrillogenic derivatives. Physiol Rev 77, 1081-1132.

Mattsson, N., Blennow, K., and Zetterberg, H. (2009). CSF biomarkers: pinpointing Alzheimer pathogenesis. Ann. N. Y. Acad. Sci. 1180, 28-35.

McCullagh, C.D. (2001). Risk factors for dementia. Advances in Psychiatric Treatment 7, 24-31.

McKhann, G. (1984). Clinical diagnosis of alzheimer's disease. Report of the NINCDSADRDA work group under the auspices of department of health and human services task force on alzheimer's disease. Neurology 34, 939-944.

McKhann, G.M., Knopman, D.S., Chertkow, H., Hyman, B.T., Jack, C.R., Kawas, C.H., Klunk, W.E., Koroshetz, W.J., Manly, J.J., and Mayeux, R., et al. (2011). The diagnosis of dementia due to Alzheimer's disease: recommendations from the National Institute on Aging-Alzheimer's Association workgroups on diagnostic guidelines for Alzheimer's disease. Alzheimers Dement 7, 263-269.

McLean, C.A., Cherny, R.A., Fraser, F.W., Fuller, S.J., Smith, M.J., Beyreuther, K., Bush, A.I., and Masters, C.L. (1999). Soluble pool of Abeta amyloid as a determinant of severity of neurodegeneration in Alzheimer's disease. Ann. Neurol. 46, 860-866.

McShane, R., Areosa Sastre, A., and Minakaran, N. (2006). Memantine for dementia. Cochrane Database Syst Rev, CD003154.

Medway, C., and Morgan, K. (2014). Review: The genetics of Alzheimer's disease; putting flesh on the bones. Neuropathol. Appl. Neurobiol. 40, 97-105.

Mega, M.S., Cummings, J.L., Fiorello, T., and Gornbein, J. (1996). The spectrum of behavioral changes in Alzheimer's disease. Neurology 46, 130-135.

Metzker, M.L. (2010). Sequencing technologies - the next generation. Nat Rev Genet 11, 31-46.

Milla, M.A., Butler, E., Huete, A.R., Wilson, C.F., Anderson, O., and Gustafson, J.P. (2002). Expressed sequence tag-based gene expression analysis under aluminum stress in rye. Plant Physiol 130, 1706-1716.

Miller, B.R., Dorner, J.L., Shou, M., Sari, Y., Barton, S.J., Sengelaub, D.R., Kennedy, R.T., and Rebec, G.V. (2008). Up-regulation of GLT1 expression increases glutamate uptake and attenuates the Huntington's disease phenotype in the R6/2 mouse. Neuroscience 153, 329-337.

Miller, D.L., Papayannopoulos, I.A., Styles, J., Bobin, S.A., Lin, Y.Y., Biemann, K., and lqbal, K. (1993). Peptide compositions of the cerebrovascular and senile plaque core amyloid deposits of Alzheimer's disease. Arch. Biochem. Biophys. 301, 41-52.

Miners, J.S., Baig, S., Tayler, H., Kehoe, P.G., and Love, S. (2009). Neprilysin and insulindegrading enzyme levels are increased in Alzheimer disease in relation to disease severity. J. Neuropathol. Exp. Neurol. 68, 902-914.

Miravalle, L., Calero, M., Takao, M., Roher, A.E., Ghetti, B., and Vidal, R. (2005). Aminoterminally truncated Abeta peptide species are the main component of cotton wool plaques. Biochemistry 44, 10810-10821.

Moechars, D., Lorent, K., Strooper, B. de, Dewachter, I., and van Leuven, F. (1996). Expression in brain of amyloid precursor protein mutated in the alpha-secretase site 
causes disturbed behavior, neuronal degeneration and premature death in transgenic mice. EMBO J. 15, 1265-1274.

Mohamed, A., and Posse de Chaves, Elena (2011). A $\beta$ internalization by neurons and glia. Int J Alzheimers Dis 2011, 127984.

Mongeau, R., Miller, G.A., Chiang, E., and Anderson, D.J. (2003). Neural correlates of competing fear behaviors evoked by an innately aversive stimulus. J Neurosci $23,3855-$ 3868 .

Moran, P.M., Higgins, L.S., Cordell, B., and Moser, P.C. (1995). Age-related learning deficits in transgenic mice expressing the 751-amino acid isoform of human beta-amyloid precursor protein. Proc. Natl. Acad. Sci. U.S.A. 92, 5341-5345.

Mori, C., Spooner, E.T., Wisniewsk, K.E., Wisniewski, T.M., Yamaguch, H., Saido, T.C., Tolan, D.R., Selkoe, D.J., and Lemere, C.A. (2002). Intraneuronal Abeta42 accumulation in Down syndrome brain. Amyloid 9, 88-102.

Mori, H., Takio, K., Ogawara, M., and Selkoe, D.J. (1992). Mass spectrometry of purified amyloid beta protein in Alzheimer's disease. J Biol Chem 267, 17082-17086.

Morris, R. (1984). Developments of a water-maze procedure for studying spatial learning in the rat. J. Neurosci. Methods 11, 47-60.

Moser, M.B., Moser, E.I., Forrest, E., Andersen, P., and Morris, R.G. (1995). Spatial learning with a minislab in the dorsal hippocampus. Proc Natl Acad Sci U S A 92, 96979701.

Mullan, M., Crawford, F., Axelman, K., Houlden, H., Lilius, L., Winblad, B., and Lannfelt, L. (1992). A pathogenic mutation for probable Alzheimer's disease in the APP gene at the Nterminus of beta-amyloid. Nat Genet 1, 345-347.

Murayama, K.S., Kametani, F., Tabira, T., and Araki, W. (2007). A novel monoclonal antibody specific for the amino-truncated beta-amyloid Abeta5-40/42 produced from caspase-cleaved amyloid precursor protein. J Neurosci Methods 161, 244-249.

Mutz, K.-O., Heilkenbrinker, A., Lönne, M., Walter, J.-G., and Stahl, F. (2013). Transcriptome analysis using next-generation sequencing. Curr. Opin. Biotechnol. 24, $22-$ 30.

Nagalakshmi, U., Wang, Z., Waern, K., Shou, C., Raha, D., Gerstein, M., and Snyder, M. (2008). The transcriptional landscape of the yeast genome defined by RNA sequencing. Science 320, 1344-1349.

Naj, A.C., Jun, G., Beecham, G.W., Wang, L.-S., Vardarajan, B.N., Buros, J., Gallins, P.J., Buxbaum, J.D., Jarvik, G.P., and Crane, P.K., et al. (2011). Common variants at MS4A4/MS4A6E, CD2AP, CD33 and EPHA1 are associated with late-onset Alzheimer's disease. Nat. Genet. 43, 436-441.

Nash, S., Henry, J.D., McDonald, S., Martin, I., Brodaty, H., and Peek-O'Leary, M.-A. (2007). Cognitive disinhibition and socioemotional functioning in Alzheimer's disease. J Int Neuropsychol Soc 13, 1060-1064.

Naslund, J., Haroutunian, V., Mohs, R., Davis, K.L., Davies, P., Greengard, P., and Buxbaum, J.D. (2000). Correlation between elevated levels of amyloid beta-peptide in the brain and cognitive decline. JAMA 283, 1571-1577.

Naslund, J., Schierhorn, A., Hellman, U., Lannfelt, L., Roses, A.D., Tjernberg, L.O., Silberring, J., Gandy, S.E., Winblad, B., and Greengard, P. (1994). Relative abundance of Alzheimer A beta amyloid peptide variants in Alzheimer disease and normal aging. Proc Natl Acad Sci U S A 91, 8378-8382. 
Nikolic, M., Dudek, H., Kwon, Y.T., Ramos, Y.F., and Tsai, L.H. (1996). The cdk5/p35 kinase is essential for neurite outgrowth during neuronal differentiation. Genes Dev. 10, 816-825.

Nilsberth, C., Westlind-Danielsson, A., Eckman, C.B., Condron, M.M., Axelman, K., Forsell, C., Stenh, C., Luthman, J., Teplow, D.B., and Younkin, S.G., et al. (2001). The 'Arctic' APP mutation (E693G) causes Alzheimer's disease by enhanced Abeta protofibril formation. Nat Neurosci 4, 887-893.

Nilselid, A.-M., Davidsson, P., Nägga, K., Andreasen, N., Fredman, P., and Blennow, K. (2006). Clusterin in cerebrospinal fluid: analysis of carbohydrates and quantification of native and glycosylated forms. Neurochem. Int. 48, 718-728.

Nixon, R.A. (2005). Endosome function and dysfunction in Alzheimer's disease and other neurodegenerative diseases. Neurobiol Aging 26, 373-382.

Nölle, A., van Haastert, E.S., Zwart, R., Hoozemans, J.J.M., and Scheper, W. (2013). Ubiquilin 2 is not associated with tau pathology. PLoS ONE 8, e76598.

Oakley, H., Cole, S.L., Logan, S., Maus, E., Shao, P., Craft, J., Guillozet-Bongaarts, A., Ohno, M., Disterhoft, J., and van Eldik, L., et al. (2006). Intraneuronal beta-Amyloid Aggregates, Neurodegeneration, and Neuron Loss in Transgenic Mice with Five Familial Alzheimer's Disease Mutations: Potential Factors in Amyloid Plaque Formation. Journal of Neuroscience 26, 10129-10140.

O'Brien, R.J., and Wong, P.C. (2011). Amyloid precursor protein processing and Alzheimer's disease. Annu. Rev. Neurosci. 34, 185-204.

Oddo, S., Caccamo, A., Shepherd, J.D., Murphy, M.P., Golde, T.E., Kayed, R., Metherate, R., Mattson, M.P., Akbari, Y., and LaFerla, F.M. (2003). Triple-transgenic model of Alzheimer's disease with plaques and tangles: intracellular Abeta and synaptic dysfunction. Neuron 39, 409-421.

Ohno, M. (2009). Failures to reconsolidate memory in a mouse model of Alzheimer's disease. Neurobiol Learn Mem 92, 455-459.

Ohno, M., Chang, L., Tseng, W., Oakley, H., Citron, M., Klein, W.L., Vassar, R., and Disterhoft, J.F. (2006). Temporal memory deficits in Alzheimer's mouse models: rescue by genetic deletion of BACE1. Eur J Neurosci 23, 251-260.

Olton, D.S., Becker, J.T., and Handelmann, G.E. (1979). Hippocampus, space, and memory. Behav Brain Sci 2, 313-322.

Orgogozo, J.-M., Rigaud, A.-S., Stoffler, A., Mobius, H.-J., and Forette, F. (2002). Efficacy and Safety of Memantine in Patients With Mild to Moderate Vascular Dementia: A Randomized, Placebo-Controlled Trial (MMM 300). Stroke 33, 1834-1839.

Padurariu, M., Ciobica, A., Mavroudis, I., Fotiou, D., and Baloyannis, S. (2012). Hippocampal neuronal loss in the CA1 and CA3 areas of Alzheimer's disease patients. Psychiatr Danub 24, 152-158.

Parsons, C.G., Danysz, W., Dekundy, A., and Pulte, I. (2013). Memantine and cholinesterase inhibitors: complementary mechanisms in the treatment of Alzheimer's disease. Neurotox Res 24, 358-369.

Parsons, C.G., Stöffler, A., and Danysz, W. (2007). Memantine: a NMDA receptor antagonist that improves memory by restoration of homeostasis in the glutamatergic system--too little activation is bad, too much is even worse. Neuropharmacology 53, 699723.

Perrin, R.J., Fagan, A.M., and Holtzman, D.M. (2009). Multimodal techniques for diagnosis and prognosis of Alzheimer's disease. Nature 461, 916-922. 
Peskind, E.R., Potkin, S.G., Pomara, N., Ott, B.R., Graham, S.M., Olin, J.T., and McDonald, S. (2006). Memantine treatment in mild to moderate Alzheimer disease: a 24week randomized, controlled trial. Am J Geriatr Psychiatry 14, 704-715.

Petersen, R.C. (2004). Mild cognitive impairment as a diagnostic entity. J. Intern. Med. 256, 183-194.

Pfaffl, M.W., Horgan, G.W., and Dempfle, L. (2002). Relative expression software tool (REST) for group-wise comparison and statistical analysis of relative expression results in real-time PCR. Nucleic Acids Res. 30, e36.

Pham, E., Crews, L., Ubhi, K., Hansen, L., Adame, A., Cartier, A., Salmon, D., Galasko, D., Michael, S., and Savas, J.N., et al. (2010). Progressive accumulation of amyloid-beta oligomers in Alzheimer's disease and in amyloid precursor protein transgenic mice is accompanied by selective alterations in synaptic scaffold proteins. Shank1. FEBS J. 277, 3051-3067.

Phillips, R.G., and LeDoux, J.E. (1992). Differential contribution of amygdala and hippocampus to cued and contextual fear conditioning. Behav. Neurosci. 106, 274-285.

Pike, C.J., Cummings, B.J., and Cotman, C.W. (1995a). Early association of reactive astrocytes with senile plaques in Alzheimer's disease. Experimental Neurology 132, $172-$ 179.

Pike, C.J., Overman, M.J., and Cotman, C.W. (1995b). Amino-terminal Deletions Enhance Aggregation of Amyloid Peptides in Vitro. Journal of Biological Chemistry 270, 2389523898.

Pimplikar, S.W. (2009). Reassessing the Amyloid Cascade Hypothesis of Alzheimer's Disease. Int J Biochem Cell Biol 41, 1261-1268.

Plassman, B.L., Havlik, R.J., Steffens, D.C., Helms, M.J., Newman, T.N., Drosdick, D., Phillips, C., Gau, B.A., Welsh-Bohmer, K.A., and Burke, J.R., et al. (2000). Documented head injury in early adulthood and risk of Alzheimer's disease and other dementias. Neurology 55, 1158-1166.

Portelius, E., Bogdanovic, N., Gustavsson, M.K., Volkmann, I., Brinkmalm, G., Zetterberg, H., Winblad, B., and Blennow, K. (2010). Mass spectrometric characterization of brain amyloid beta isoform signatures in familial and sporadic Alzheimer's disease. Acta Neuropathol 120, 185-193.

Portelius, E., Zetterberg, H., Dean, R.A., Marcil, A., Bourgeois, P., Nutu, M., Andreasson, U., Siemers, E., Mawuenyega, K.G., and Sigurdson, W.C., et al. (2012). Amyloid- $\beta(1-$ 15/16) as a marker for $y$-secretase inhibition in Alzheimer's disease. J. Alzheimers Dis. 31, 335-341.

Prelli, F., Castaño, E., Glenner, G.G., and Frangione, B. (1988). Differences between vascular and plaque core amyloid in Alzheimer's disease. J. Neurochem. 51, 648-651.

Prince, M., Cullen, M., and Mann, A. (1994). Risk factors for Alzheimer's disease and dementia: a case-control study based on the MRC elderly hypertension trial. Neurology 44, 97-104.

Probst, A., Gotz, J., Wiederhold, K.H., Tolnay, M., Mistl, C., Jaton, A.L., Hong, M., Ishihara, T., Lee, V.M., and Trojanowski, J.Q., et al. (2000). Axonopathy and amyotrophy in mice transgenic for human four-repeat tau protein. Acta Neuropathol 99, 469-481.

Prüßing, K., Voigt, A., and Schulz, J.B. (2013). Drosophila melanogaster as a model organism for Alzheimer's disease. Mol Neurodegener 8, 35.

Pugh, P.L., Richardson, J.C., Bate, S.T., Upton, N., and Sunter, D. (2007). Non-cognitive behaviours in an APP/PS1 transgenic model of Alzheimer's disease. Behav Brain Res $178,18-28$. 
Puzzo, D., Lee, L., Palmeri, A., Calabrese, G., and Arancio, O. (2014). Behavioral assays with mouse models of Alzheimer's disease: practical considerations and guidelines. Biochem Pharmacol 88, 450-467.

Qin, S., Hu, X.-Y., Xu, H., and Zhou, J.-N. (2004). Regional alteration of synapsin I in the hippocampal formation of Alzheimer's disease patients. Acta Neuropathol. 107, 209-215.

Qiu, C., Winblad, B., Marengoni, A., Klarin, I., Fastbom, J., and Fratiglioni, L. (2006). Heart failure and risk of dementia and Alzheimer disease: a population-based cohort study. Arch. Intern. Med. 166, 1003-1008.

Rademakers, R., Sleegers, K., Theuns, J., van den Broeck, M., Bel Kacem, S., Nilsson, L.-G., Adolfsson, R., van Duijn, C.M., van Broeckhoven, C., and Cruts, M. (2005). Association of cyclin-dependent kinase 5 and neuronal activators p35 and p39 complex in early-onset Alzheimer's disease. Neurobiol. Aging 26, 1145-1151.

Rebeck, G.W., Hoe, H.-S., and Moussa, C.E.-H. (2010). Amyloid1-42 Gene Transfer Model Exhibits Intraneuronal Amyloid, Gliosis, Tau Phosphorylation, and Neuronal Loss. J Biol Chem 285, 7440-7446.

Reilly, J.F., Games, D., Rydel, R.E., Freedman, S., Schenk, D., Young, W.G., Morrison, J.H., and Bloom, F.E. (2003). Amyloid deposition in the hippocampus and entorhinal cortex: quantitative analysis of a transgenic mouse model. Proc. Natl. Acad. Sci. U.S.A. 100, 4837-4842.

Reitz, C., and Mayeux, R. (2014). Alzheimer disease: epidemiology, diagnostic criteria, risk factors and biomarkers. Biochem Pharmacol 88, 640-651.

Renner, M., Lacor, P.N., Velasco, P.T., Xu, J., Contractor, A., Klein, W.L., and Triller, A. (2010). Deleterious effects of amyloid beta oligomers acting as an extracellular scaffold for mGluR5. Neuron 66, 739-754.

Ring, S., Weyer, S.W., Kilian, S.B., Waldron, E., Pietrzik, C.U., Filippov, M.A., Herms, J., Buchholz, C., Eckman, C.B., and Korte, M., et al. (2007). The secreted beta-amyloid precursor protein ectodomain APPs alpha is sufficient to rescue the anatomical, behavioral, and electrophysiological abnormalities of APP-deficient mice. J Neurosci 27, 7817-7826.

Risso, D., Schwartz, K., Sherlock, G., and Dudoit, S. (2011). GC-content normalization for RNA-Seq data. BMC Bioinformatics 12, 480.

Riviere, S., Gillette-Guyonnet, S., Voisin, T., Reynish, E., Andrieu, S., Lauque, S., Salva, A., Frisoni, G., Nourhashemi, F., and Micas, M., et al. (2001). A nutritional education program could prevent weight loss and slow cognitive decline in Alzheimer's disease. $\mathrm{J}$ Nutr Health Aging 5, 295-299.

Roberts, B.R., Ryan, T.M., Bush, A.I., Masters, C.L., and Duce, J.A. (2012). The role of metallobiology and amyloid- $\beta$ peptides in Alzheimer's disease. J. Neurochem. 120 Suppl 1, 149-166.

Rogaev, E.I., Sherrington, R., Rogaeva, E.A., Levesque, G., Ikeda, M., Liang, Y., Chi, H., Lin, C., Holman, K., and Tsuda, T. (1995). Familial Alzheimer's disease in kindreds with missense mutations in a gene on chromosome 1 related to the Alzheimer's disease type 3 gene. Nature 376, 775-778.

Rogers, J., Cooper, N.R., Webster, S., Schultz, J., McGeer, P.L., Styren, S.D., Civin, W.H., Brachova, L., Bradt, B., and Ward, P. (1992). Complement activation by betaamyloid in Alzheimer disease. Proc. Natl. Acad. Sci. U.S.A. 89, 10016-10020.

Rogers, S.L. (1998). Donepezil Improves Cognition and Global Function in Alzheimer DiseaseA 15-Week, Double-blind, Placebo-Controlled Study. Arch Intern Med 158, 1021. 
Roychaudhuri, R., Yang, M., Hoshi, M.M., and Teplow, D.B. (2009). Amyloid beta-protein assembly and Alzheimer disease. J. Biol. Chem. 284, 4749-4753.

Rubio-Perez, J.M., and Morillas-Ruiz, J.M. (2012). A review: inflammatory process in Alzheimer's disease, role of cytokines. ScientificWorldJournal 2012, 756357.

Rumble, B., Retallack, R., Hilbich, C., Simms, G., Multhaup, G., Martins, R., Hockey, A., Montgomery, P., Beyreuther, K., and Masters, C.L. (1989). Amyloid A4 protein and its precursor in Down's syndrome and Alzheimer's disease. N Engl J Med 320, 1446-1452.

Runz, H., Rietdorf, J., Tomic, I., Bernard, M. de, Beyreuther, K., Pepperkok, R., and Hartmann, T. (2002). Inhibition of intracellular cholesterol transport alters presenilin localization and amyloid precursor protein processing in neuronal cells. J Neurosci 22, 1679-1689.

Russo, C., Saido, T.C., DeBusk, L.M., Tabaton, M., Gambetti, P., and Teller, J.K. (1997). Heterogeneity of water-soluble amyloid $\beta$-peptide in Alzheimer's disease and Down's syndrome brains. FEBS Letters 409, 411-416.

Ryan, N.S., and Rossor, M.N. (2010). Correlating familial Alzheimer's disease gene mutations with clinical phenotype. Biomark Med 4, 99-112.

Saido, T.C., Iwatsubo, T., Mann, D.M., Shimada, H., Ihara, Y., and Kawashima, S. (1995). Dominant and differential deposition of distinct beta-amyloid peptide species, A beta N3(pE), in senile plaques. Neuron 14, 457-466.

Salkovic-Petrisic, M., Tribl, F., Schmidt, M., Hoyer, S., and Riederer, P. (2006). Alzheimerlike changes in protein kinase $B$ and glycogen synthase kinase-3 in rat frontal cortex and hippocampus after damage to the insulin signalling pathway. J Neurochem 96, 10051015.

Sandbrink, R., Masters, C.L., and Beyreuther, K. (1996). APP gene family. Alternative splicing generates functionally related isoforms. Ann N Y Acad Sci 777, 281-287.

Sanders, M.J., Wiltgen, B.J., and Fanselow, M.S. (2003). The place of the hippocampus in fear conditioning. Animal Models of Anxiety Disorders 463, 217-223.

Sarasa, M., and Pesini, P. (2009). Natural Non-Trasgenic Animal Models for Research in Alzheimers Disease. CAR 6, 171-178.

Scarmeas, N., Hadjigeorgiou, G.M., Papadimitriou, A., Dubois, B., Sarazin, M., Brandt, J., Albert, M., Marder, K., Bell, K., and Honig, L.S., et al. (2004). Motor signs during the course of Alzheimer disease. Neurology 63, 975-982.

Schaeffer, E.L., Figueiro, M., and Gattaz, W.F. (2011). Insights into Alzheimer disease pathogenesis from studies in transgenic animal models. Clinics (Sao Paulo) 66, 45-54.

Scheff, S.W., DeKosky, S.T., and Price, D.A. (1990). Quantitative assessment of cortical synaptic density in Alzheimer's disease. Neurobiology of Aging 11, 29-37.

Scheff, S.W., and Price, D.A. (1993). Synapse loss in the temporal lobe in Alzheimer's disease. Ann Neurol 33, 190-199.

Scheff, S.W., Price, D.A., Schmitt, F.A., DeKosky, S.T., and Mufson, E.J. (2007). Synaptic alterations in CA1 in mild Alzheimer disease and mild cognitive impairment. Neurology 68, 1501-1508.

Scheuner, D., Eckman, C., Jensen, M., Song, X., Citron, M., Suzuki, N., Bird, T.D., Hardy, J., Hutton, M., and Kukull, W., et al. (1996). Secreted amyloid $\beta$-protein similar to that in the senile plaques of Alzheimer's disease is increased in vivo by the presenilin 1 and 2 and APP mutations linked to familial Alzheimer's disease. Nat Med 2, 864-870. 
Schilling, S., Hoffmann, T., Manhart, S., Hoffmann, M., and Demuth, H.-U. (2004). Glutaminyl cyclases unfold glutamyl cyclase activity under mild acid conditions. FEBS Lett 563, 191-196.

Schilling, S., Zeitschel, U., Hoffmann, T., Heiser, U., Francke, M., Kehlen, A., Holzer, M., Hutter-Paier, B., Prokesch, M., and Windisch, M., et al. (2008). Glutaminyl cyclase inhibition attenuates pyroglutamate Abeta and Alzheimer's disease-like pathology. Nat Med 14, 1106-1111.

Schlenzig, D., Manhart, S., Cinar, Y., Kleinschmidt, M., Hause, G., Willbold, D., Funke, S.A., Schilling, S., and Demuth, H.-U. (2009). Pyroglutamate formation influences solubility and amyloidogenicity of amyloid peptides. Biochemistry 48, 7072-7078.

Schmand, B., Walstra, G., Lindeboom, J., Teunisse, S., and Jonker, C. (2000). Early detection of Alzheimer's disease using the Cambridge Cognitive Examination (CAMCOG). Psychol Med 30, 619-627.

Schmitz, C., and Hof, P.R. (2005). Design-based stereology in neuroscience. Neuroscience 130, 813-831.

Schmitz, C., Rutten, B.P.F., Pielen, A., Schäfer, S., Wirths, O., Tremp, G., Czech, C., Blanchard, V., Multhaup, G., and Rezaie, P., et al. (2004). Hippocampal neuron loss exceeds amyloid plaque load in a transgenic mouse model of Alzheimer's disease. Am. J. Pathol. 164, 1495-1502.

Schupf, N. (2002). Genetic and host factors for dementia in Down's syndrome. The British Journal of Psychiatry 180, 405-410.

Schuur, M., Ikram, M.A., van Swieten, J.C., Isaacs, A., Vergeer-Drop, J.M., Hofman, A., Oostra, B.A., Breteler, M.M.B., and van Duijn, C.M. (2011). Cathepsin D gene and the risk of Alzheimer's disease: a population-based study and meta-analysis. Neurobiol. Aging 32, 1607-1614.

Selkoe, D.J. (2011). Alzheimer's disease. Cold Spring Harb Perspect Biol 3.

Selkoe, D.J., Abraham, C.R., Podlisny, M.B., and Duffy, L.K. (1986). Isolation of lowmolecular-weight proteins from amyloid plaque fibers in Alzheimer's disease. $J$ Neurochem 46, 1820-1834.

Selkoe, D.J., Podlisny, M.B., Joachim, C.L., Vickers, E.A., Lee, G., Fritz, L.C., and Oltersdorf, T. (1988). Beta-amyloid precursor protein of Alzheimer disease occurs as 110to 135-kilodalton membrane-associated proteins in neural and nonneural tissues. Proc Natl Acad Sci U S A 85, 7341-7345.

Selwood, S.P., Parvathy, S., Cordell, B., Ryan, H.S., Oshidari, F., Vincent, V., Yesavage, J., Lazzeroni, L.C., and Murphy, G.M. (2009). Gene expression profile of the PDAPP mouse model for Alzheimer's disease with and without Apolipoprotein E. Neurobiol. Aging 30, 574-590.

Sergeant, N., Bombois, S., Ghestem, A., Drobecq, H., Kostanjevecki, V., Missiaen, C., Wattez, A., David, J.-P., Vanmechelen, E., and Sergheraert, C., et al. (2003). Truncated beta-amyloid peptide species in pre-clinical Alzheimer's disease as new targets for the vaccination approach. J Neurochem 85, 1581-1591.

Serrano-Pozo, A., Frosch, M.P., Masliah, E., and Hyman, B.T. (2011). Neuropathological alterations in Alzheimer disease. Cold Spring Harb Perspect Med 1, a006189.

Seshadri, S., Fitzpatrick, A.L., Ikram, M.A., Destefano, A.L., Gudnason, V., Boada, M., Bis, J.C., Smith, A.V., Carassquillo, M.M., and Lambert, J.C., et al. (2010). Genome-wide analysis of genetic loci associated with Alzheimer disease. JAMA 303, 1832-1840.

Shankar, G.M., Li, S., Mehta, T.H., Garcia-Munoz, A., Shepardson, N.E., Smith, I., Brett, F.M., Farrell, M.A., Rowan, M.J., and Lemere, C.A., et al. (2008). Amyloid-beta protein 
dimers isolated directly from Alzheimer's brains impair synaptic plasticity and memory. Nat. Med. 14, 837-842.

Shapira, R., Austin, G.E., and Mirra, S.S. (1988). Neuritic Plaque Amyloid in Alzheimer's Disease Is Highly Racemized. J Neurochem 50, 69-74.

Sharma, M., Krüger, R., and Gasser, T. (2014). From genome-wide association studies to next-generation sequencing: lessons from the past and planning for the future. JAMA Neurol 71, 5-6.

Shen, Y., Lue, L., Yang, L., Roher, A., Kuo, Y., Strohmeyer, R., Goux, W.J., Lee, V., Johnson, G.V., and Webster, S.D., et al. (2001). Complement activation by neurofibrillary tangles in Alzheimer's disease. Neurosci. Lett. 305, 165-168.

Sherva, R., and Farrer, L.A. (2011). Power and pitfalls of the genome-wide association study approach to identify genes for Alzheimer's disease. Curr Psychiatry Rep 13, 138146.

Shiotsuki, H., Yoshimi, K., Shimo, Y., Funayama, M., Takamatsu, Y., Ikeda, K., Takahashi, R., Kitazawa, S., and Hattori, N. (2010). A rotarod test for evaluation of motor skill learning. J. Neurosci. Methods 189, 180-185.

Shukla, V., Skuntz, S., and Pant, H.C. (2012). Deregulated Cdk5 activity is involved in inducing Alzheimer's disease. Arch. Med. Res. 43, 655-662.

Sinha, S., Anderson, J.P., Barbour, R., Basi, G.S., Caccavello, R., Davis, D., Doan, M., Dovey, H.F., Frigon, N., and Hong, J., et al. (1999). Purification and cloning of amyloid precursor protein beta-secretase from human brain. Nature 402, 537-540.

Sisodia, S.S. (1992). Beta-amyloid precursor protein cleavage by a membrane-bound protease. Proc Natl Acad Sci U S A 89, 6075-6079.

Sisodia, S.S., Koo, E.H., Beyreuther, K., Unterbeck, A., and Price, D.L. (1990). Evidence that beta-amyloid protein in Alzheimer's disease is not derived by normal processing. Science 248, 492-495.

Sivanandam, T.M., and Thakur, M.K. (2012). Traumatic brain injury: a risk factor for Alzheimer's disease. Neurosci Biobehav Rev 36, 1376-1381.

Skovronsky, D.M., Doms, R.W., and Lee, V.M.-Y. (1998). Detection of a Novel Intraneuronal Pool of Insoluble Amyloid ? Protein that Accumulates with Time in Culture. $\mathrm{J}$ Cell Biol 141, 1031-1039.

Slattery, C., Beck, J., Harper, L., Adamson, G., Abdi, Z., Uphill, J., Campbell, T., Druyeh, R., Mahoney, C., and Rohrer, J., et al. (2014). Trem2 variants increase risk of typical early-onset Alzheimer's disease but not of prion or frontotemporal dementia. J. Neurol. Neurosurg. Psychiatr. 85, e3.

Sleegers, K., Brouwers, N., Gijselinck, I., Theuns, J., Goossens, D., Wauters, J., DelFavero, J., Cruts, M., van Duijn, Cornelia M, and van Broeckhoven, C. (2006). APP duplication is sufficient to cause early onset Alzheimer's dementia with cerebral amyloid angiopathy. Brain 129, 2977-2983.

Slunt, H.H., Thinakaran, G., Koch, C. von, Lo, A.C., Tanzi, R.E., and Sisodia, S.S. (1994). Expression of a ubiquitous, cross-reactive homologue of the mouse beta-amyloid precursor protein (APP). J Biol Chem 269, 2637-2644.

Snowdon, D.A. (2003). Healthy aging and dementia: findings from the Nun Study. Ann Intern Med 139, 450-454.

Snyder, S.E., and Salton, S.R. (1998). Expression of VGF mRNA in the adult rat central nervous system. J. Comp. Neurol. 394, 91-105. 
Sola, C., Mengod, G., Probst, A., and Palacios, J.M. (1993). Differential regional and cellular distribution of beta-amyloid precursor protein messenger RNAs containing and lacking the Kunitz protease inhibitor domain in the brain of human, rat and mouse. Neuroscience 53, 267-295.

Sperling, R.A., Aisen, P.S., Beckett, L.A., Bennett, D.A., Craft, S., Fagan, A.M., Iwatsubo, T., Jack, C.R., Kaye, J., and Montine, T.J., et al. (2011). Toward defining the preclinical stages of Alzheimer's disease: recommendations from the National Institute on AgingAlzheimer's Association workgroups on diagnostic guidelines for Alzheimer's disease. Alzheimers Dement 7, 280-292.

Stein, T.D., and Johnson, J.A. (2002). Lack of neurodegeneration in transgenic mice overexpressing mutant amyloid precursor protein is associated with increased levels of transthyretin and the activation of cell survival pathways. J. Neurosci. 22, 7380-7388.

Stenh, C., Nilsberth, C., Hammarback, J., Engvall, B., Naslund, J., and Lannfelt, L. (2002). The Arctic mutation interferes with processing of the amyloid precursor protein. Neuroreport 13, 1857-1860.

Stieren, E.S., El Ayadi, A., Xiao, Y., Siller, E., Landsverk, M.L., Oberhauser, A.F., Barral, J.M., and Boehning, D. (2011). Ubiquilin-1 is a molecular chaperone for the amyloid precursor protein. J. Biol. Chem. 286, 35689-35698.

Stover, K.R., and Brown, R.E. (2012). Age-related changes in visual acuity, learning and memory in the APPswe/PS1dE9 mouse model of Alzheimer's disease. Behav. Brain Res. $231,75-85$.

Strittmatter, W.J., Saunders, A.M., Schmechel, D., Pericak-Vance, M., Enghild, J., Salvesen, G.S., and Roses, A.D. (1993). Apolipoprotein E: high-avidity binding to betaamyloid and increased frequency of type 4 allele in late-onset familial Alzheimer disease. Proc Natl Acad Sci U S A 90, 1977-1981.

Strooper, B. de, Iwatsubo, T., and Wolfe, M.S. (2012). Presenilins and Y-secretase: structure, function, and role in Alzheimer Disease. Cold Spring Harb Perspect Med 2, a006304.

Sturchler-Pierrat, C., Abramowski, D., Duke, M., Wiederhold, K.H., Mistl, C., Rothacher, S., Ledermann, B., Burki, K., Frey, P., and Paganetti, P.A., et al. (1997). Two amyloid precursor protein transgenic mouse models with Alzheimer disease-like pathology. Proc Natl Acad Sci U S A 94, 13287-13292.

Su, J.H., Cummings, B.J., and Cotman, C.W. (1998). Plaque biogenesis in brain aging and Alzheimer's disease. II. Progressive transformation and developmental sequence of dystrophic neurites. Acta Neuropathol 96, 463-471.

Südhof, T.C. (1990). The structure of the human synapsin I gene and protein. J. Biol. Chem. 265, 7849-7852.

Sultan, M., Schulz, M.H., Richard, H., Magen, A., Klingenhoff, A., Scherf, M., Seifert, M., Borodina, T., Soldatov, A., and Parkhomchuk, D., et al. (2008). A global view of gene activity and alternative splicing by deep sequencing of the human transcriptome. Science 321, 956-960.

Sutherland, G.T., Janitz, M., and Kril, J.J. (2011). Understanding the pathogenesis of Alzheimer's disease: will RNA-Seq realize the promise of transcriptomics? J. Neurochem. 116, 937-946.

Suzuki, N., Cheung, T.T., Cai, X.D., Odaka, A., Otvos, L., JR, Eckman, C., Golde, T.E., and Younkin, S.G. (1994). An increased percentage of long amyloid beta protein secreted by familial amyloid beta protein precursor (beta APP717) mutants. Science 264, 13361340. 
Szendrei, G.I., Fabian, H., Mantsch, H.H., Lovas, S., Nyeki, O., Schon, I., and Otvos, L., JR (1994). Aspartate-bond isomerization affects the major conformations of synthetic peptides. Eur J Biochem 226, 917-924.

Takahashi, K., Rochford, Christian D P, and Neumann, H. (2005). Clearance of apoptotic neurons without inflammation by microglial triggering receptor expressed on myeloid cells2. J. Exp. Med. 201, 647-657.

Takahashi, R.H., Almeida, C.G., Kearney, P.F., Yu, F., Lin, M.T., Milner, T.A., and Gouras, G.K. (2004). Oligomerization of Alzheimer's beta-amyloid within processes and synapses of cultured neurons and brain. J Neurosci 24, 3592-3599.

Takahashi, R.H., Capetillo-Zarate, E., Lin, M.T., Milner, T.A., and Gouras, G.K. (2013). Accumulation of intraneuronal $\beta$-amyloid 42 peptides is associated with early changes in microtubule-associated protein 2 in neurites and synapses. PLoS ONE 8, e51965.

Takahashi, R.H., Milner, T.A., Li, F., Nam, E.E., Edgar, M.A., Yamaguchi, H., Beal, M.F., $\mathrm{Xu}$, H., Greengard, P., and Gouras, G.K. (2002). Intraneuronal Alzheimer Abeta42 Accumulates in Multivesicular Bodies and Is Associated with Synaptic Pathology. Am J Pathol 161, 1869-1879.

Takemura, R., Nakata, T., Okada, Y., Yamazaki, H., Zhang, Z., and Hirokawa, N. (1996). mRNA expression of KIF1A, KIF1B, KIF2, KIF3A, KIF3B, KIF4, KIF5, and cytoplasmic dynein during axonal regeneration. J. Neurosci. 16, 31-35.

Tambo, K., Yamaguchi, T., Kobayashi, K., Terauchi, E., Ichi, I., and Kojo, S. (2013). Racemization of the aspartic acid residue of amyloid-beta peptide by a radical reaction. Biosci Biotechnol Biochem 77, 416-418.

Tamura, B.K., Masaki, K.H., and Blanchette, P. (2007). Weight loss in patients with Alzheimer's disease. J Nutr Elder 26, 21-38.

Tan, T.C., Valova, V.A., Malladi, C.S., Graham, M.E., Berven, L.A., Jupp, O.J., Hansra, G., McClure, S.J., Sarcevic, B., and Boadle, R.A., et al. (2003). Cdk5 is essential for synaptic vesicle endocytosis. Nat. Cell Biol. 5, 701-710.

Tanila, H. (2012). Wading pools, fading memories-place navigation in transgenic mouse models of Alzheimer's disease. Front Aging Neurosci 4, 11.

Tariot, P.N., Farlow, M.R., Grossberg, G.T., Graham, S.M., McDonald, S., and Gergel, I. (2004). Memantine treatment in patients with moderate to severe Alzheimer disease already receiving donepezil: a randomized controlled trial. JAMA 291, 317-324.

Terry, A.V., and Buccafusco, J.J. (2003). The cholinergic hypothesis of age and Alzheimer's disease-related cognitive deficits: recent challenges and their implications for novel drug development. J. Pharmacol. Exp. Ther. 306, 821-827.

Tesseur, I., van Dorpe, J., Bruynseels, K., Bronfman, F., Sciot, R., van Lommel, A., and van Leuven, F. (2000). Prominent axonopathy and disruption of axonal transport in transgenic mice expressing human apolipoprotein E4 in neurons of brain and spinal cord. Am J Pathol 157, 1495-1510.

Thakker-Varia, S., and Alder, J. (2009). Neuropeptides in depression: role of VGF. Behav. Brain Res. 197, 262-278.

Thal, D.R., Rub, U., Orantes, M., and Braak, H. (2002). Phases of A beta-deposition in the human brain and its relevance for the development of AD. Neurology 58, 1791-1800.

Tharp, W.G., and Sarkar, I.N. (2013). Origins of amyloid-B. BMC Genomics 14, 290.

Thomas, P., and Fenech, M. (2007). A review of genome mutation and Alzheimer's disease. Mutagenesis 22, 15-33. 
Thonberg, H., Fallström, M., Björkström, J., Schoumans, J., Nennesmo, I., and Graff, C. (2011). Mutation screening of patients with Alzheimer disease identifies APP locus duplication in a Swedish patient. BMC Res Notes 4, 476.

Tomidokoro, Y., Lashley, T., Rostagno, A., Neubert, T.A., Bojsen-Moller, M., Braendgaard, H., Plant, G., Holton, J., Frangione, B., and Revesz, T., et al. (2005). Familial Danish dementia: co-existence of Danish and Alzheimer amyloid subunits (ADan and Abeta) in the absence of compact plaques. J Biol Chem 280, 36883-36894.

Tomiyama, T., Asano, S., Furiya, Y., Shirasawa, T., Endo, N., and Mori, H. (1994). Racemization of Asp23 residue affects the aggregation properties of Alzheimer amyloid beta protein analogues. J Biol Chem 269, 10205-10208.

Tomiyama, T., Matsuyama, S., Iso, H., Umeda, T., Takuma, H., Ohnishi, K., Ishibashi, K., Teraoka, R., Sakama, N., and Yamashita, T., et al. (2010). A mouse model of amyloid beta oligomers: their contribution to synaptic alteration, abnormal tau phosphorylation, glial activation, and neuronal loss in vivo. J Neurosci 30, 4845-4856.

Tosto, G., and Reitz, C. (2013). Genome-wide association studies in Alzheimer's disease: a review. Curr Neurol Neurosci Rep 13, 381.

Tuppo, E.E., and Arias, H.R. (2005). The role of inflammation in Alzheimer's disease. Int. J. Biochem. Cell Biol. 37, 289-305.

Twine, N.A., Janitz, K., Wilkins, M.R., and Janitz, M. (2011). Whole transcriptome sequencing reveals gene expression and splicing differences in brain regions affected by Alzheimer's disease. PLoS ONE 6, e16266.

van Bakel, H., Nislow, C., Blencowe, B.J., and Hughes, T.R. (2010). Most "dark matter" transcripts are associated with known genes. PLoS Biol. 8, e1000371.

van Dam, D., D'Hooge, R., Staufenbiel, M., van Ginneken, C., van Meir, F., and De Deyn, Peter P (2003). Age-dependent cognitive decline in the APP23 model precedes amyloid deposition. Eur J Neurosci 17, 388-396.

van den Pol, A.N., Bina, K., Decavel, C., and Ghosh, P. (1994). VGF expression in the brain. J. Comp. Neurol. 347, 455-469.

Van Nostrand, W E, Melchor, J.P., Cho, H.S., Greenberg, S.M., and Rebeck, G.W. (2001). Pathogenic effects of D23N lowa mutant amyloid beta -protein. J Biol Chem 276, 32860-32866.

Van Vickle, G D, Esh, C.L., Kokjohn, T.A., Patton, R.L., Kalback, W.M., Luehrs, D.C., Beach, T.G., Newel, A.J., Lopera, F., and Ghetti, B., et al. (2008). Presenilin-1 280Glu-Ala mutation alters C-terminal APP processing yielding longer abeta peptides: implications for Alzheimer's disease. Mol Med 14, 184-194.

Vassar, R., Bennett, B.D., Babu-Khan, S., Kahn, S., Mendiaz, E.A., Denis, P., Teplow, D.B., Ross, S., Amarante, P., and Loeloff, R., et al. (1999). Beta-secretase cleavage of Alzheimer's amyloid precursor protein by the transmembrane aspartic protease BACE. Science 286, 735-741.

Velculescu, V.E., Zhang, L., Vogelstein, B., and Kinzler, K.W. (1995). Serial analysis of gene expression. Science 270, 484-487.

Verkhratsky, A., Olabarria, M., Noristani, H.N., Yeh, C.-Y., and Rodriguez, J.J. (2010). Astrocytes in Alzheimer's disease. Neurotherapeutics 7, 399-412.

Verwer, R.W.H., Sluiter, A.A., Balesar, R.A., Baayen, J.C., Noske, D.P., Dirven, C.M.F., Wouda, J., van Dam, A.M., Lucassen, P.J., and Swaab, D.F. (2007). Mature astrocytes in the adult human neocortex express the early neuronal marker doublecortin. Brain 130, 3321-3335. 
Vidal, M., Morris, R., Grosveld, F., and Spanopoulou, E. (1990). Tissue-specific control elements of the Thy-1 gene. EMBO J. 9, 833-840.

Villemagne, V.L., Fodero-Tavoletti, M.T., Pike, K.E., Cappai, R., Masters, C.L., and Rowe, C.C. (2008). The ART of loss: Abeta imaging in the evaluation of Alzheimer's disease and other dementias. Mol Neurobiol 38, 1-15.

Villemagne, V.L., Pike, K.E., Chetelat, G., Ellis, K.A., Mulligan, R.S., Bourgeat, P., Ackermann, U., Jones, G., Szoeke, C., and Salvado, O., et al. (2011). Longitudinal assessment of Abeta and cognition in aging and Alzheimer disease. Ann Neurol 69, 181192.

Visser, P.J., Scheltens, P., and Verhey, F R J (2005). Do MCl criteria in drug trials accurately identify subjects with predementia Alzheimer's disease? J. Neurol. Neurosurg. Psychiatr. 76, 1348-1354.

Viswanathan, J., Haapasalo, A., Böttcher, C., Miettinen, R., Kurkinen, K.M.A., Lu, A., Thomas, A., Maynard, C.J., Romano, D., and Hyman, B.T., et al. (2011). Alzheimer's disease-associated ubiquilin-1 regulates presenilin-1 accumulation and aggresome formation. Traffic 12, 330-348.

Vogel, C., and Marcotte, E.M. (2012). Insights into the regulation of protein abundance from proteomic and transcriptomic analyses. Nat. Rev. Genet. 13, 227-232.

Vorhees, C.V., and Williams, M.T. (2006). Morris water maze: procedures for assessing spatial and related forms of learning and memory. Nat Protoc 1, 848-858.

Wada, H., Nakajoh, K., Satoh-Nakagawa, T., Suzuki, T., Ohrui, T., Arai, H., and Sasaki, $\mathrm{H}$. (2001). Risk factors of aspiration pneumonia in Alzheimer's disease patients. Gerontology 47, 271-276.

Waldemar, G., Dubois, B., Emre, M., Georges, J., McKeith, I.G., Rossor, M., Scheltens, P., Tariska, P., and Winblad, B. (2007). Recommendations for the diagnosis and management of Alzheimer's disease and other disorders associated with dementia: EFNS guideline. Eur. J. Neurol. 14, e1-26.

Walf, A.A., and Frye, C.A. (2007). The use of the elevated plus maze as an assay of anxiety-related behavior in rodents. Nat Protoc 2, 322-328.

Wallace, J.I., Schwartz, R.S., LaCroix, A.Z., Uhlmann, R.F., and Pearlman, R.A. (1995). Involuntary weight loss in older outpatients: incidence and clinical significance. J Am Geriatr Soc 43, 329-337.

Wallin, A.K., Wattmo, C., and Minthon, L. (2011). Galantamine treatment in Alzheimer's disease: response and long-term outcome in a routine clinical setting. Neuropsychiatr Dis Treat 7, 565-576.

Walsh, D.M., and Selkoe, D.J. (2007). A beta oligomers - a decade of discovery. J. Neurochem. 101, 1172-1184.

Walsh, D.M., Tseng, B.P., Rydel, R.E., Podlisny, M.B., and Selkoe, D.J. (2000). The Oligomerization of Amyloid $\beta$-Protein Begins Intracellularly in Cells Derived from Human Brain. Biochemistry 39, 10831-10839.

Wang, C., Sun, B., Zhou, Y., Grubb, A., and Gan, L. (2012). Cathepsin B degrades amyloid- $\beta$ in mice expressing wild-type human amyloid precursor protein. J. Biol. Chem. 287, 39834-39841.

Wang, Z., Gerstein, M., and Snyder, M. (2009). RNA-Seq: a revolutionary tool for transcriptomics. Nat. Rev. Genet. 10, 57-63.

Wasco, W., Gurubhagavatula, S., Paradis, M.D., Romano, D.M., Sisodia, S.S., Hyman, B.T., Neve, R.L., and Tanzi, R.E. (1993). Isolation and characterization of APLP2 
encoding a homologue of the Alzheimer's associated amyloid beta protein precursor. Nat Genet 5, 95-100.

Watt, N.T., Whitehouse, I.J., and Hooper, N.M. (2010). The role of zinc in Alzheimer's disease. Int J Alzheimers Dis 2011, 971021.

Webster, S.J., Bachstetter, A.D., and Van Eldik, Linda J (2013). Comprehensive behavioral characterization of an APP/PS-1 double knock-in mouse model of Alzheimer's disease. Alzheimers Res Ther 5, 28.

Wehner, J.M., and Radcliffe, R.A. (2004). Cued and contextual fear conditioning in mice. Curr Protoc Neurosci Chapter 8, Unit 8.5C.

Welander, H., Frånberg, J., Graff, C., Sundström, E., Winblad, B., and Tjernberg, L.O. (2009). Abeta43 is more frequent than Abeta40 in amyloid plaque cores from Alzheimer disease brains. J. Neurochem. 110, 697-706.

West, M.J. (1993). Regionally specific loss of neurons in the aging human hippocampus. Neurobiol Aging 14, 287-293.

West, M.J., Kawas, C.H., Stewart, W.F., Rudow, G.L., and Troncoso, J.C. (2004). Hippocampal neurons in pre-clinical Alzheimer's disease. Neurobiol Aging 25, 1205-1212.

West, M.J., Slomianka, L., and Gundersen, H.J. (1991). Unbiased stereological estimation of the total number of neurons in thesubdivisions of the rat hippocampus using the optical fractionator. Anat $\operatorname{Rec} 231,482-497$.

Westerman, M.A., Cooper-Blacketer, D., Mariash, A., Kotilinek, L., Kawarabayashi, T., Younkin, L.H., Carlson, G.A., Younkin, S.G., and Ashe, K.H. (2002). The relationship between Abeta and memory in the Tg2576 mouse model of Alzheimer's disease. J Neurosci 22, 1858-1867.

World Health Organization. (2012). Dementia. A public health priority. Geneva, London:; Alzheimer's Disease International.

Wietrzych, M., Meziane, H., Sutter, A., Ghyselinck, N., Chapman, P.F., Chambon, P., and Krezel, W. (2005). Working memory deficits in retinoid $X$ receptor gamma-deficient mice. Learn. Mem. 12, 318-326.

Wiltfang, J., Esselmann, H., Bibl, M., Smirnov, A., Otto, M., Paul, S., Schmidt, B., Klafki, H.-W., Maler, M., and Dyrks, T., et al. (2002). Highly conserved and disease-specific patterns of carboxyterminally truncated Abeta peptides 1-37/38/39 in addition to 1-40/42 in Alzheimer's disease and in patients with chronic neuroinflammation. J Neurochem 81, 481-496.

Wiltfang, J., Esselmann, H., Cupers, P., Neumann, M., Kretzschmar, H., Beyermann, M., Schleuder, D., Jahn, H., Ruther, E., and Kornhuber, J., et al. (2001). Elevation of betaamyloid peptide 2-42 in sporadic and familial Alzheimer's disease and its generation in PS1 knockout cells. J Biol Chem 276, 42645-42657.

Wirths, O., Multhaup, G., and Bayer, T.A. (2004). A modified beta-amyloid hypothesis: intraneuronal accumulation of the beta-amyloid peptide--the first step of a fatal cascade. J. Neurochem. 91, 513-520.

Wirths, O., Weis, J., Kayed, R., Saido, T.C., and Bayer, T.A. (2007). Age-dependent axonal degeneration in an Alzheimer mouse model. Neurobiol Aging 28, 1689-1699.

Wirths, O., and Bayer, T.A. (2008). Motor impairment in Alzheimer's disease and transgenic Alzheimer's disease mouse models. Genes Brain Behav. 7 Suppl 1, 1-5.

Wirths, O., Breyhan, H., Schäfer, S., Roth, C., and Bayer, T.A. (2008). Deficits in working memory and motor performance in the APP/PS1ki mouse model for Alzheimer's disease. Neurobiol. Aging 29, 891-901. 
Wirths, O., Breyhan, H., Cynis, H., Schilling, S., Demuth, H.-U., and Bayer, T.A. (2009). Intraneuronal pyroglutamate-Abeta 3-42 triggers neurodegeneration and lethal neurological deficits in a transgenic mouse model. Acta Neuropathol. 118, 487-496.

Wirths, O., Bethge, T., Marcello, A., Harmeier, A., Jawhar, S., Lucassen, P.J., Multhaup, G., Brody, D.L., Esparza, T., and Ingelsson, M., et al. (2010). Pyroglutamate Abeta pathology in APP/PS1KI mice, sporadic and familial Alzheimer's disease cases. J Neural Transm 117, 85-96.

Wirths, O., and Bayer, T.A. (2012). Intraneuronal $A \beta$ accumulation and neurodegeneration: lessons from transgenic models. Life Sci. 91, 1148-1152.

Wirz, K.T.S., Bossers, K., Stargardt, A., Kamphuis, W., Swaab, D.F., Hol, E.M., and Verhaagen, J. (2013). Cortical beta amyloid protein triggers an immune response, but no synaptic changes in the APPswe/PS1dE9 Alzheimer's disease mouse model. Neurobiol. Aging 34, 1328-1342.

Wittnam, J.L. (2012). The contribution of N-terminally modified amyloid beta to the etiology of Alzheimer's disease. Ph.D.-Thesis, Georg-August-Universität Göttingen.

Wittnam, J.L., Portelius, E., Zetterberg, H., Gustavsson, M.K., Schilling, S., Koch, B., Demuth, H.-U., Blennow, K., Wirths, O., and Bayer, T.A. (2012). Pyroglutamate amyloid $\beta$ $(A \beta)$ aggravates behavioral deficits in transgenic amyloid mouse model for Alzheimer disease. J. Biol. Chem. 287, 8154-8162.

Wolfe, M.S. (2007). When loss is gain: reduced presenilin proteolytic function leads to increased Abeta42/Abeta40. Talking Point on the role of presenilin mutations in Alzheimer disease. EMBO Rep. 8, 136-140.

Wolfe, M.S. (2010). Structure, mechanism and inhibition of gamma-secretase and presenilin-like proteases. Biol. Chem. 391, 839-847.

Wu, Y., and Luo, Y. (2005). Transgenic C. elegans as a model in Alzheimer's research. Curr Alzheimer Res 2, 37-45.

Wu, Z.-L., Ciallella, J.R., Flood, D.G., O'Kane, T.M., Bozyczko-Coyne, D., and Savage, M.J. (2006). Comparative analysis of cortical gene expression in mouse models of Alzheimer's disease. Neurobiol. Aging 27, 377-386.

Wyss-Coray, T., Loike, J.D., Brionne, T.C., Lu, E., Anankov, R., Yan, F., Silverstein, S.C., and Husemann, J. (2003). Adult mouse astrocytes degrade amyloid-beta in vitro and in situ. Nat Med 9, 453-457.

Yamaguchi, H., Hirai, S., Morimatsu, M., Shoji, M., and Harigaya, Y. (1988a). Diffuse type of senile plaques in the brains of Alzheimer-type dementia. Acta Neuropathol. 77, 113119.

Yamaguchi, H., Hirai, S., Morimatsu, M., Shoji, M., and Ihara, Y. (1988b). A variety of cerebral amyloid deposits in the brains of the Alzheimer-type dementia demonstrated by beta protein immunostaining. Acta Neuropathol. 76, 541-549.

Yan, R., Bienkowski, M.J., Shuck, M.E., Miao, H., Tory, M.C., Pauley, A.M., Brashier, J.R., Stratman, N.C., Mathews, W.R., and Buhl, A.E., et al. (1999). Membrane-anchored aspartyl protease with Alzheimer's disease beta-secretase activity. Nature 402, 533-537.

Yang, L.-B., Lindholm, K., Yan, R., Citron, M., Xia, W., Yang, X.-L., Beach, T., Sue, L., Wong, P., and Price, D., et al. (2003). Elevated beta-secretase expression and enzymatic activity detected in sporadic Alzheimer disease. Nat Med 9, 3-4.

$\mathrm{Yu}$, J.-T., and Tan, L. (2012). The role of clusterin in Alzheimer's disease: pathways, pathogenesis, and therapy. Mol. Neurobiol. 45, 314-326. 
Zetterberg, H., and Mattsson, N. (2014). Understanding the cause of sporadic Alzheimer's disease. Expert Rev Neurother 14, 621-630.

Zhang, X.-M., Cai, Y., Xiong, K., Cai, H., Luo, X.-G., Feng, J.-C., Clough, R.W., Struble, R.G., Patrylo, P.R., and Yan, X.-X. (2009). Beta-secretase-1 elevation in transgenic mouse models of Alzheimer's disease is associated with synaptic/axonal pathology and amyloidogenesis: implications for neuritic plaque development. Eur. J. Neurosci. 30, 2271 2283.

Zhang, Y.-W., Thompson, R., Zhang, H., and Xu, H. (2011). APP processing in Alzheimer's disease. Mol Brain 4, 3.

Zheng, H., Jiang, M., Trumbauer, M.E., Sirinathsinghji, D.J., Hopkins, R., Smith, D.W., Heavens, R.P., Dawson, G.R., Boyce, S., and Conner, M.W., et al. (1995). beta-Amyloid precursor protein-deficient mice show reactive gliosis and decreased locomotor activity. Cell 81, 525-531.

Zheng, H., and Koo, E.H. (2006). The amyloid precursor protein: beyond amyloid. Mol Neurodegener 1, 5 .

Zheng, H., and Koo, E.H. (2011). Biology and pathophysiology of the amyloid precursor protein. Mol Neurodegener 6, 27. 
